\title{
SEARCH FOR CHARGINO AND NEUTRALINO AT \\ RUN II OF THE TEVATRON COLLIDER
}

\author{
A Thesis \\ Submitted to the Faculty \\ of \\ Purdue University \\ by \\ Anadi Canepa

\begin{abstract}
In Partial Fulfillment of the
\end{abstract} \\ Requirements for the Degree \\ of \\ Doctor of Philosophy
}

August 2006

Purdue University

West Lafayette, Indiana 
To my mother Silvana and father Diego. And to Zoltan. We can all wear the hat! 


\section{ACKNOWLEDGMENTS}

I would like to express a deep gratitude to my advisor, professor Daniela Bortoletto. In these years you honored me with your invaluable support and trust. I loved and appreciated your passion, your commitment and your idea of collaboration. I have no words to express the enthusiasm you can donate! I also would like to remember my undergraduate professor, Dario Bisello, who "sent me to the lake on vacation". My life has changed since then, thanks Dario (!). Thanks to you as well, dear Donatella. Near the famous lake, I joined the fabulous CDF Silicon Group, who made my dream become true. Gino and Petra (Luca and Anna now!), you became my family in the US. And talking about special people, here you are Monica: I wish the "time at Speedway" never ends! After the long and cold nights of the first Winter, I moved to Purdue where I found you, my favorite "normalista", Stefanino, always teasing me, Angelo and Ney sharing with me important changes in life and the old tenant of Ferry Street, Ahmed. Arnold and Sarka, you were waiting for me at the door of Potawatomi 15 when I got back to Fermilab: you made me believe that life is there, you just need to grab it (with discipline!). And I finally caught my dear Kim, for years I had been running after you (... your "ciabatta polesana"!). Oscar, thanks for never let me fall during the hard beginning and for your insights into physics. Else, I will miss you! Your extremely professional attitude accompanied by your sensibility made my $\mathrm{PhD}$ possible: the days spent together will remain sweetest memories. The road, full of up's and down's, was long! So I had the chance to meet Alon who taught me (tried to!) that nothing is ever a disaster; the "trilepton gang", from the John's (one is even a hidden painter!) to Vadim and Sourabh: trileptons for ever !!! Thanks

Giulia for the passionate :) journey we traveled together, and Beate, for making me fall in love with each event detected at CDF! I would like to thank Teruki and Ming for being knowledgeable guides in my research and Stephan for the constant and pa- 
tient support. A great acknowledgement to the entire CDF Collaboration: this is a dream world where curiosity, passion, commitment and freedom at the same time make our life exciting!

This work was supported by the DOE grand, DE-FG02-91ER-40681A29. 


\section{TABLE OF CONTENTS}

$\begin{array}{lll}\text { Page } & \end{array}$

LIST OF TABLES . . . . . . . . . . . . . . . . . . . ix

LIST OF FIGURES ......................... xii

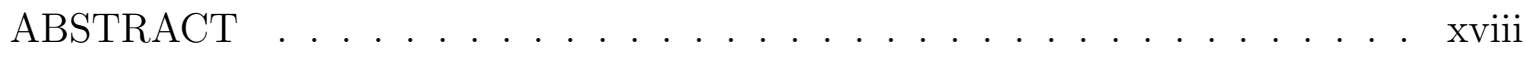

1 Introduction . . . . . . . . . . . . . . . . . . 1

2 A brief introduction to the Standard Model . . . . . . . . . . . . . . . 3

2.1 Particle content and interactions . . . . . . . . . . . . 3

2.2 Open questions in the $\mathrm{SM} \ldots \ldots \ldots 6$

3 Supersymmetry . . . . . . . . . . . . . . . . . . . . 9

3.1 SUSY Formalism . . . . . . . . . . . . . . . . . . 9

3.1 Fermions ........................... 9

3.1.2 From fermions to bosons . . . . . . . . . . . . . . . . 10

3.1.3 Particle content of the MSSM . . . . . . . . . . . . . 11

3.1.4 SUSY Lagrangian . . . . . . . . . . . . . . . . . . 13

3.1.5 SUSY breaking . . . . . . . . . . . . . . . . 14

3.1.6 Mass spectrum in the context of the Minimal Supersymmetric Standard Model (MSSM) . . . . . . . . . . . 15

3.2 Minimal supergravity . . . . . . . . . . . . . . . . . 18

3.3 Does SUSY answer the open questions in the SM? . . . . . . . . . . 19

4 Sensitivity to SUSY at the Run II of the Tevatron . . . . . . . . . . . 21

4.1 Current constraints in mSUGRA . . . . . . . . . . . . . . 22

4.1.1 Cold dark matter . . . . . . . . . . . . . . . . . 22

4.1.2 Rare decays and low energy constraints . . . . . . . . . . 25

4.1.3 EWK Precision measurements . . . . . . . . . . . . . . . 27

4.1.4 Direct searches for Chargino . . . . . . . . . . . . . . 29 
4.2 SUSY Signature at Tevatron . . . . . . . . . . . . . . . . 30

4.3 Sensitivity . . . . . . . . . . . . . . . . . . 33

4.3.1 "SPS1a" Benchmark Line . . . . . . . . . . . . . . . . . . . . 34

4.3.2 mSUGRA parameter space for $\mu>0 \ldots . . \ldots 37$

4.3.3 Bulk of the mSUGRA parameter space . . . . . . . . . . . 39

4.3.4 Cosmologically preferred regions . . . . . . . . . . . . . 39

5 The Tevatron and the CDF Experiment . . . . . . . . . . . . . 43

5.1 The Tevatron . . . . . . . . . . . . . . . . 43

5.1.1 Production and boosting of protons . . . . . . . . . . 45

5.1 .2 Main Injector . . . . . . . . . . . . . . . . 46

5.1.3 Production and cooling of antiprotons . . . . . . . . . . 46

5.1.4 Tevatron ........................... 47

5.2 Collider Detector at Fermilab . . . . . . . . . . . . . . 48

5.2.1 Central Tracking System . . . . . . . . . . . 50

5.2 .2 Time of Flight Detector . . . . . . . . . . . . 53

5.2 .3 Calorimetry system . . . . . . . . . . . . . . 54

5.2 .4 Muon system . . . . . . . . . . . . . 56

5.2.5 Trigger and data acquisition system . . . . . . . . . . . . 59

5.2.6 Triggers for data analysis . . . . . . . . . . . . . . 62

5.2.7 Luminosity measurement . . . . . . . . . . . . . . . . . . 63

5.2.8 Collected luminosity for data analysis . . . . . . . . . . . . 64

6 Event reconstruction . . . . . . . . . . . . . . . . 65

6.1 Tracks ............................... 65

6.2 Primary vertex f. . . . . . . . . . . . . . . 69

6.3 Muons ............................ 69

6.4 Electrons. . . . . . . . . . . . . . . . . 76

6.5 Jets . . . . . . . . . . . . . . . . . . 81

6.6 Missing Transverse Energy . . . . . . . . . . . . . . . . 83 
7 Standard Model backgrounds . . . . . . . . . . . . . . . 85

7.1 Drell-Yan production ..................... 85

7.2 Diboson production . . . . . . . . . . . . . 86

7.3 Top pair production ...................... 89

7.4 Misidentified leptons . . . . . . . . . . . . . . . 91

7.5 Calculation of the number of expected events . . . . . . . . . 95

8 The search . . . . . . . . . . . . . . . . . . 96

8.1 Blind box analysis technique . . . . . . . . . . . . . 96

8.2 SUSY Monte Carlo sample . . . . . . . . . . . . . . . . 97

8.3 Event preselection . . . . . . . . . . . . . . . . 98

8.4 Topological cuts . . . . . . . . . . . . . . . . . . 100

8.5 Signal Box . . . . . . . . . . . . . . . . 106

8.6 Understanding the SM backgrounds . . . . . . . . . . . . . 109

8.6.1 Control regions with two muons . . . . . . . . . . . . 109

8.6.2 Control regions with one muon and one electron . . . . . . . . 117

8.6.3 Control regions with three leptons . . . . . . . . . . . 118

9 Discussion of the results and conclusions . . . . . . . . . . . . . 122

9.1 Systematic uncertainties . . . . . . . . . . . . . . . 122

9.2 Data in the signal box . . . . . . . . . . . . . . 124

9.3 Interpretation of the results . . . . . . . . . . . . . 130

9.4 Results of the searches for chargino and neutralino at CDF . . . . . . 134

9.5 Interpretation of the CDF results . . . . . . . . . . . . . 137

9.6 Conclusions . . . . . . . . . . . . . . . . . . . . 143

A Trigger efficiency . . . . . . . . . . . . . . . . . . . 144

A.1 L1_CMU1.5_PT1.5 . . . . . . . . . . . . . . . 146

A.2 L1_CMUP6_PT4 . . . . . . . . . . . . . . . . . . . . 147

A.3 L2_TRK8 . . . . . . . . . . . . . . . . . . . . . . 149

B Muon identification efficiency . . . . . . . . . . . . . . 150 
C Photon conversion tagging efficiency . . . . . . . . . . . . . . . . 152

LIST OF REFERENCES . . . . . . . . . . . . . . . . . . . 162 


\section{LIST OF TABLES}

Table

Page

2.1 List of the Standard Model particles. The left handed leptons and quarks are weak-isospin $\left(S U(2)_{L}\right)$ doublets. The right handed leptons and quarks are weak-isospin $\left(S U(2)_{L}\right)$ singlets. Each quark is a color $\left(S U(3)_{C}\right)$ triplet. All other particles are color $\left(S U(3)_{C}\right)$ singlets. $)$. 7

3.1 Chiral multiplets in the MSSM. . . . . . . . . . . . . . . 12

3.2 Vector multiplets in the MSSM. . . . . . . . . . . . . . . . . 12

5.1 Trigger path efficiency (the uncertainty is statistical only) . . . . . 63

6.1 Muon reconstruction efficiency measured in data and in simulated data (statistical uncertainty only). . . . . . . . . . . . . . . . . 72

6.2 Muon Identification selection criteria. . . . . . . . . . . . . . . . . . 74

6.3 High $p_{T}$ Muon identification efficiency (statistical uncertainty only). . 76

6.4 Medium $p_{T}$ Muon identification efficiency with tight isolation requirement as measured in [58] (statistical uncertainty only). . . . . . . . 76

6.5 Medium $p_{T}$ Muon identification efficiency with loose isolation requirement as measured in [58] (statistical uncertainty only). . . . . . . . . 77

6.6 Identification selection criteria applied to electrons . . . . . . . . . . . 81

6.7 Electron identification efficiency measured in data and in simulated data (statistical uncertainty only). . . . . . . . . . . .

8.1 Number of events with at least two identified muons before $\left(N_{b}\right)$ and after $\left(N_{a}\right)$ applying the requirement of the azimuthal distance between the missing transverse energy and the dimuon system. . . . . . . .

8.2 Number of events with at least two muons before $\left(N_{b}\right)$ and after $\left(N_{a}\right)$ applying the requirement of the azimuthal distance between the missing transverse energy and the jets with $E_{T}>5 \mathrm{GeV}$. . . . . . . 100

8.3 Definition of the analysis channels based on the lepton selection. . . . 101

8.4 Number of events with at least three leptons. . . . . . . . . . . . . 102

8.5 Number of events with at least three leptons after the invariant mass requirement in the $\mu-\mu$ channel. . . . . . . . . . . . . . . . . . . 102 
8.6 Number of events with at least three leptons after the invariant mass and jet multiplicity requirements in the $\mu-\mu$ channel . . . . . . . . 104

8.7 Number of events with at least three leptons after the invariant mass, the jet multiplicity and the missing transverse energy requirements in the $\mu-\mu$ channel. . . . . . . . . . . . . . . . . . . . . . 108

8.8 Number of expected events in the $\mu-\mu$ and the $\mu$-e signal boxes. . 108

8.9 Cross sections of the process $Z \rightarrow \mu \mu$ (statistical uncertainty and systematic uncertainty due to luminosity measurement quoted in Section $9.1)$

8.10 Comparison between the number of expected and observed events in events with two muons . . . . . . . . . . . . . . 117

8.11 Comparison between the number of expected and observed events in events with one muon and one CTE. . . . . . . . . . . . . . . . 118

8.12 Comparison between the number of expected and observed events in events with one muon and one PLE/PHE. . . . . . . . . . . . . . 118

8.13 Comparison between the number of expected and observed events in events with two muon and one lepton.

8.14 Comparison between the number of expected and observed events in events with one muon, one CTE and one lepton. . . . . . . . . . . . 121

8.15 Comparison between the number of expected and observed events in events with one muon, one PLE/PHE and one lepton. . . . . . . . . 121

9.1 Systematic uncertainties on the NLO production cross sections. . . . 124

9.2 Systematic uncertainties in the $\mu-\mu$ channel. . . . . . . . . . . . 125

9.3 Systematic uncertainties in the $\mu$-CLE/CTE channel. . . . . . . . 125

9.4 Systematic uncertainties in the $\mu$-PLE/PHE channel. . . . . . . . 126

9.5 Number of expected and observed events in the the three analysis channels. . . . . . . . . . . . . . . . . . . . . 126

9.6 Description the event observed in the $\mu-\mu$ channel. . . . . . . . . . 127

9.7 Correlation between the systematic uncertainties among the different channels. . . . . . . . . . . . . . . . . . .

9.8 Trilepton analyses; left column, eel selection in high $E_{T}$ single electron data sample; middle column, $\mu \mu \ell$ in low $p_{T}$ dilepton data sample; left column, ee + track selection in low $p_{T}$ dilepton data sample. . . . . . 
9.9 Systematic uncertainties of the trilepton analyses (the first number is the SUSY signal, the second the SM background). . . . . . . . . . . . 136

9.10 LS analyses: left column $e^{ \pm} e^{ \pm}$selection in high $E_{T}$ single electron data sample; middle column, $\mu^{ \pm} \mu^{ \pm}$in high $p_{T}$ single muon data sample; left column, $e^{ \pm} \mu^{ \pm}$selection in high $E_{T}$ single electron data sample and high $p_{T}$ single muon data sample. . . . . . . . . . . . . . . . . . 137

9.11 Systematic uncertainties of the LS analyses (the first number is the SUSY signal, the second the SM background). . . . . . . . . . . . 137

B.1 Muon identification efficiency in data and MC. Note that the identification efficiency of medium $p_{T}$ muons does not include the loose isolation efficiency calculated in [90] and the stub matching requirement for the CMU is $\Delta X<3 \mathrm{~cm}$. . . . . . . . . . . . . . . . . . . . . . 151

C.1 Conversion removal efficiency in Data and MC. . . . . . . . . . . . . 159 


\section{LIST OF FIGURES}

Figure $\quad$ Page

2.1 Schematic view of the Higgs potential with $\mu^{2}<0$ and $\lambda>0$. . . 5

2.2 Correction to $\mathrm{m}_{H}^{2}$ from a loop containing a Dirac fermion $f$ with mass $m_{f} . \Lambda_{U V}$ is the ultraviolet momentum. . . . . . . . . . . 8

3.1 Running of gauge coupling constants in the SM and in the MSSM frameworks as a function of the energy scale denoted $\mu[9] . \quad \ldots . .20$

4.1 Evolution of the soft supersymmetry breaking mass terms in the Universal MSSM. . . . . . . . . . . . . . . . . . . 21

4.2 SUSY production cross section as a function of the super-particle mass. 22

4.3 Neutralinos annihilation via slepton. . . . . . . . . . . . . 23

4.4 Higgs coannihilation . . . . . . . . . . . . . . . . 24

4.5 Stau coannihilation . . . . . . . . . . . . . . . . 24

4.6 Higgs contributions to the $B_{s}$ mixing. . . . . . . . . . . . . 25

4.7 Higgs contributions to the $B_{s} \rightarrow \mu \mu$. . . . . . . . . . . 26

$4.8 b \rightarrow s \gamma$ via $W$ loop. . . . . . . . . . . . . . . . . . . . . . . 26

4.9 Preferred regions in mSUGRA parameter space. The dotted lines label different values of the Higgs mass. . . . . . . . . . . . . . . . . 28

4.10 Combined likelihood $\chi^{2}$ as a function of the supersymmetric particle masses. . . . . . . . . . . . . . . . . . .

4.11 Boundary of the focus point in the mSUGRA space for different top masses. . . . . . . . . . . . . . . . . . 30

4.12 Obtained $\tilde{\chi_{1}^{ \pm}}$mass limit as a function of the sneutrino mass. . . . . . 31

4.13 Chargino-Neutralino associated production at $\mathrm{p} \overline{\mathrm{p}}$ collider. . . . . . . . 31

4.14 Chargino and neutralino decay modes. . . . . . . . . . . . . . 33

4.15 Superparticle mass as a function of the gaugino unified mass $m_{\frac{1}{2}}$. . . 35

4.16 Cross section as a function of the chargino mass. . . . . . . . . . . 35 
4.17 Chargino branching ratios as a function of the gaugino unified mass $m_{\frac{1}{2}}$ (Red cross symbols, chargino into $\tilde{\tau_{1}} \nu$; green solid line chargino into $f f^{\prime} \tilde{\chi_{1}^{0}}$; blue line with square symbols, chargino into $\left.W{\tilde{\chi_{1}^{0}}}^{0}\right) . \quad$. 36

4.18 Cross section times branching ratio as a function of the Chargino mass $m_{\frac{1}{2}} \ldots \ldots \ldots \ldots \ldots \ldots \ldots \ldots \ldots \ldots \ldots \ldots \ldots \ldots$

4.19 Cross section times branching ratio as a function of the gaugino and scalar unified mass $\left(\tan \beta=5 ; A_{0}=0 ; \mu>0\right)$. The LEP excluded regions are not investigated. The $\tau$ is not forced to decay leptonically. . . . . 38

$4.20 \sigma \times B R$ as a function of $A_{0} \ldots \ldots \ldots$. . . . . . . . . . 40

$4.21 \sigma \times B R$ as a function of the chargino mass. . . . . . . . . . . . . . 41

$4.22 \sigma\left(q \bar{q} \rightarrow \tilde{\chi}_{1}^{ \pm} \tilde{\chi}_{2}^{0}\right) \times B R\left(\tilde{\chi}_{1}^{ \pm} \tilde{\chi}_{2}^{0} \rightarrow \ell \ell \ell \tilde{\chi}_{1}^{0}\right)$ as a function of the chargino mass. 42

5.1 A schematic drawing of the Fermilab accelerator complex. . . . . . . 44

5.2 Average number of interactions per bunch crossing as a function of the instantaneous luminosity for different beam conditions. . . . . . . . . 45

5.3 Tevatron Performance through Spring 2006. . . . . . . . . . . . . 48

5.4 CDF Run II detector view. . . . . . . . . . . . . . . . . . . . . . . 49

5.5 Coordinate system. . . . . . . . . . . . . . . . . 49

5.6 CDF Run II tracking system view. . . . . . . . . . . . . . . . 50

5.7 Schematic $r-\phi$ (left) and $r-z$ (right) views the Run II CDF silicon detector. . . . . . . . . . . . . . . 52

$5.8 r-z$ view of the plug calorimeter. . . . . . . . . . . . 56

5.9 Location of the muon upgrade components in azimuth $\phi$ and pseudorapidity $\eta$ for Run I. The gray cross-hatched region indicates the currently uninstrumented regions of CMP and CMX. Miniskirt and keystone, described in the text, are now instrumented. . . . . . . . .

5.10 CDF pipelines and buffers trigger architecture (left). Block diagram of the Run II trigger system. . . . . . . . . . . . . . . . . .

6.1 Silicon efficiency vs $p_{T}$. Open squares: COT track intersects three active $r-\phi$ layers of SVXII. Open triangles: COT track intersects three active stereo layers of SVXII. Closed squares: Silicon $r-\phi$ hits found in three layers of SVXII. Closed triangles: Silicon stereo hits found in three layers of SVXII . . . . . . . . . . . . . . 67

6.2 Identification variables of tracks. . . . . . . . . . . . . . . 70 
Figure $\quad$ Page

6.3 Muon reconstruction efficiency as a function of $\eta$. . . . . . . . . . . 72

6.4 Identification variables of muons. . . . . . . . . . . . . . 75

6.5 High $p_{T}$ muon identification efficiency as a function of $\eta$. . . . . . . 76

6.6 Identification variables of electrons. . . . . . . . . . . . . . 80

6.7 Missing transverse energy in Drell-Yan events at generation level, reconstruction level and after the corrections. . . . . . . . . . . . . 84

7.1 LO and NLO Drell-Yan production . . . . . . . . . . . . . 86

$7.2 \mathrm{k}$-factor for Drell-Yan production as a function of the invariant mass. $\quad 87$

7.3 Correlation between the invariant mass and the azimuthal distance between muons in Drell-Yan events. . . . . . . . . . . . . . . 87

7.4 LO Diboson production . . . . . . . . . . . . . . . . 88

7.5 NLO Diboson production . . . . . . . . . . . . . . . . 88

7.6 Opposite sign muon invariant mass distribution in diboson events (WW, $\left.\mathrm{WZ}^{*}, \mathrm{ZZ}\right) . \ldots \ldots \ldots . \ldots \ldots$

7.7 Missing transverse energy in diboson events (WW, WZ $\left.\gamma^{*}, \mathrm{ZZ}\right) \ldots$. . . 89

7.8 LO $t \bar{t}$ production production . . . . . . . . . . . . . . . . . . . 90

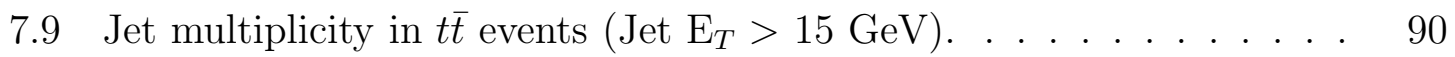

7.10 Fake rate of Tight Central Electrons . . . . . . . . . . . . . . . 93

7.11 Fake rate of CMUP Muons . . . . . . . . . . . . . . . . 94

7.12 Distribution of fakeable objects in the search data sample. . . . . . . 94

8.1 Azimuthal distance between the missing transverse energy and each identified jet in events with two muons. . . . . . . . . . . . . . . 99

8.2 Invariant mass distribution of opposite sign muons in vents with at least three leptons. . . . . . . . . . . . . . . . . . . . . 103

8.3 Number of jets with $E_{T}>20 \mathrm{GeV}$ in events with at least three leptons after the invariant mass requirement. . . . . . . . . . . . . . 105

8.4 Sensitivity as a function of the missing transverse energy threshold in the $\mu-\mu$ channel. . . . . . . . . . . . . . . . . . . . . 106 
Figure $\quad$ Page

8.5 Missing transverse energy in events with at least three leptons after the invariant mass and jet multiplicity requirements. The data are not plotted in the region where the missing transverse energy is larger than $15 \mathrm{GeV}$. . . . . . . . . . . . . . . . . . 107

8.6 Sketch of the control regions. Control regions A, E, G and I (A2, F, H and $\mathrm{J}$ ) are such that the jet cut is satisfied (fails). Each control region is examined with two and three leptons. Control region A is examined only with two leptons since it becomes the signal box if three leptons are required.

8.7 Invariant mass of opposite sign muons in dimuon events in the region

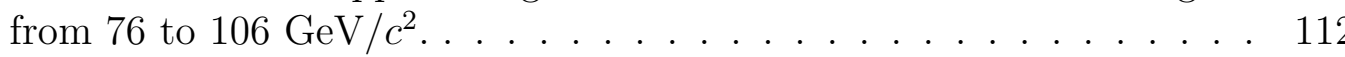

8.8 Kinematical distributions for dimuon events with invariant mass in the

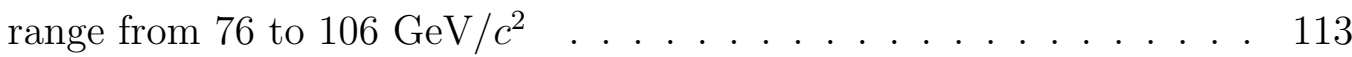

8.9 Kinematical distributions for dimuon events outside the Z mass window.114

8.10 Control region G. . . . . . . . . . . . . . . . . . . . . . . . . . . . . 115

8.11 Control region A. . . . . . . . . . . . . . . . . . . . 116

8.12 Kinematical distributions in $\mu$-e events. . . . . . . . . . . . . . . . . 119

9.1 $r \phi$ view. A cut of $p_{T} \geq 1 \mathrm{GeV}$ is applied to the tracks in the event. . 128

$9.2 \mathrm{Z}$ view. A cut of $p_{T} \geq 1 \mathrm{GeV}$ is applied to tracks in the event. . . . . 129

9.3 The excluded cross section limit is plotted as a function of the chargino mass in the mSUGRA like scenario. . . . . . . . . . . . . . . . 133

9.4 The excluded cross section limit is plotted as a function of the chargino mass in the mSUGRA like scenario with the assumption of observing 0 events (dashed line). . . . . . . . . . . . . . . . 134

9.5 The excluded cross section limit is plotted as a function of the chargino mass in the mSUGRA like scenario combining the three analysis (solid line) and for the $\mu-\mu$ channel only (dashed line). . . . . . . . . .

9.6 Acceptance of LS and trilepton analyses as a function of the chargino mass. . . . . . . . . . . . . . . . . . . . . . . . . 138

9.7 The excluded cross section limit is plotted as a function of the chargino mass in the mSUGRA scenario. The expected limit (black dashed line) and the theory curve (red solid line), with its uncertainty (red dotted lines) is also shown. The yellow and cyan bands represent the \pm 1 and \pm 2 sigmas uncertainties on the expected limit. The expected exclusion limit corresponds to a chargino mass of approximately $117 \mathrm{GeV} / c^{2}$. 
Figure Page

9.8 Masses as a function of the mSUGRA parameter $m_{\frac{1}{2}}$. . . . . . . 140

9.9 Branching ratios as a function of the mSUGRA parameter $m_{\frac{1}{2}} \ldots \ldots$

9.10 The excluded cross section limit plotted as a function of the chargino mass in a mSUGRA-like scenario with slepton mixing suppressed. The expected limit (black solid line), and the theory curve (red solid line) with its uncertainty (red dotted lines) is also shown. The yellow and cyan bands represent the \pm 1 and \pm 2 sigmas uncertainties on the expected limit. The observed limit on the mass of the chargino is approximately $127 \mathrm{GeV} / c^{2}$ and the expected one is approximately 140 $\mathrm{GeV} / c^{2}$. . . . . . . . . . . . . . . . . . . 142

A.1 Transverse momentum distribution of muons. . . . . . . . . . . . . 145

A.2 Matching efficiencies as a function of the offline muon $p_{T} \ldots \ldots$. . . 148

A.3 L1 Trigger efficiency. . . . . . . . . . . . . . . . . . . . . . . . 148

A.4 Trigger efficiency as a function of the offline muon $p_{T} \ldots \ldots$. . . . . 149

C.1 Energy sharing between electron and positron in photon conversions. 153

C.2 Method of selecting conversion candidates and determining correct and incorrect side. . . . . . . . . . . . . . . . . . . . . . . . 154

C.3 Local $\Delta \mathrm{Z}$ between the seed electron and the CES partner cluster for the correct (circles) and incorrect (red line) side. . . . . . . . . . . . . 155

C.4 Background subtracted $\Delta \cot \theta$ and $D_{x y}$ for Data and MC. . . . . . . 156

C.5 Background subtracted Data and MC distributions of photon conversion candidates: seed electron transverse energy (top left), CES partner cluster energy (top right), seed electron $E / p$ (middle left), seed electron fractional isolation (middle right), $\Delta \cot \theta$ (bottom left), $\mathrm{D}_{x y}$ (bottom right). . . . . . . . . . . . . . . . .

C.6 A "bremsstrahlung" event where an electron radiates a photon. The photon will be on the correct side. . . . . . . . . . . . . . . 157

C.7 Two "trident" events where an electron radiates a photon that converts. 158

C.8 CES clusters for photon conversions belonging to the "non CES sample": the blue line represents the seed electron, the red lines represent different scenario for the CES partner cluster (Monte Carlo events). . 159

C.9 Efficiency for the "non CES sample" as a function of the partner track minimum $\mathrm{p}_{T}$. The line represents the efficiency of the "CES sample". 
C.10 Conversion removal scale factor as a function of the seed electron $\mathrm{E}_{T}$. Only the first $4 \mathrm{E}_{T}$ bins are used in the fit. The last bin is not used since the mean $\mathrm{E}_{T}=25 \mathrm{GeV}$ does not equal the bin center and would bias the fit. . . . . . . . . . . . . . . . . . . 160 


\begin{abstract}
Canepa, Anadi Ph.D., Purdue University, August, 2006. Search for Chargino and Neutralino at Run II of the Tevatron Collider. Major Professor: D. Bortoletto.

In this dissertation we present a search for the associated production of charginos and neutralinos, the supersymmetric partners of the Standard Model bosons. We analyze a data sample representing $745 \mathrm{pb}^{-1}$ of integrated luminosity collected by the CDF experiment at the p $\overline{\mathrm{p}}$ Tevatron collider. We compare the Standard Model predictions with the observed data selecting events with three leptons and missing transverse energy. Finding no excess, we combine the results of our search with similar analyses carried out at CDF and set an upper limit on the chargino mass in SUSY scenarios.
\end{abstract}




\section{Introduction}

\section{What is the nature of the Universe?}

The quest to discover the fundamental laws of Nature animates humankind since the times of the ancient Greeks. But it is the $21^{\text {st }}$ century when particle physics, the ultimate science disclosing the secrets of the Universe, triumphs. With the most sophisticated probes on Earth, the accelerators, particle physics discovers the fundamental constituents of the visible matter and the physical laws at higher and higher energies. Today's knowledge manifests itself in the elegant theory known as the Standard Model. At the time when the human endeavor is gratified by countless experimental confirmations of the theory, cosmological data reveal that the SM accounts for only a tiny fraction of the whole Universe. A mysterious energy, dark energy, permeates the empty space accelerating the universe expansion. An elusive form of matter, dark matter likely originated at the Big Bang holds the Universe together. Humankind is posed the question again, what is the nature of the Universe? If it is fascinating that the SM is merely founded on symmetries, it is amazing that its extension called Supersymmetry might shed light on the Dark Universe.

In this dissertation we describe the search for the superpartners of the Standard Model bosons, the charginos and neutralinos. The superparticles are expected to be among the lightest superpartners and their leptonic decays gives rise to the most

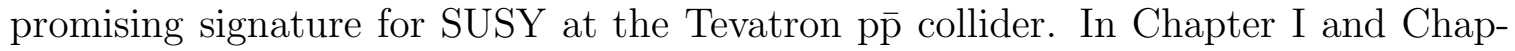
ter II we briefly introduce the Standard Model and Supersymmetry. In Chapter III we describe the current experimental constraints on SUSY models. Chapter IV and Chapter $\mathrm{V}$ are devoted to the experimental apparatus and the details of the event reconstruction. The Standard Model backgrounds are described in Chapter VI. Fi- 
nally Chapters VII and Chapter VIII present the search and the interpretation of the results. 


\section{A brief introduction to the Standard Model}

The Standard Model of particle physics is a quantum field theory which describes the strong, weak, and electromagnetic fundamental forces, as well as the fundamental particles that form the matter. The SM emerged in the early '70 and its predictions have been confirmed with high accuracy in the last decades. Nevertheless, there are open questions which stimulate a rich search for physics beyond the SM. In the current chapter we review the main ideas of the SM concluding with its unresolved issues.

\subsection{Particle content and interactions}

The SM [1] [2] [3] is founded on the concept of symmetry since the interactions it describes are uniquely defined from a symmetry group. To construct a complete quantum gauge field we

- build the Lagrangian for the free matter fields,

- impose the local invariance under a particular gauge group,

- identify the gauge interaction terms,

- add the free gauge fields to the Lagrangian.

In the SM the matter is described in terms of initially massless Dirac fermions grouped into three generations of quarks and leptons shown in Equation 2.1 to Equation 2.3.

$$
\begin{aligned}
& 1^{\text {st }} \text { family : } L_{e}=\left(\begin{array}{c}
\nu_{e} \\
e
\end{array}\right)_{L}, R_{e}=e_{R}, L_{u}=\left(\begin{array}{c}
u \\
d^{\prime}
\end{array}\right)_{L}, R_{u}=u_{R}, R_{d}=d_{R} \\
& 2^{\text {nd }} \text { family }: L_{\mu}=\left(\begin{array}{c}
\nu_{\mu} \\
\mu
\end{array}\right)_{L}, R_{\mu}=\mu_{R}, L_{c}=\left(\begin{array}{c}
c \\
s^{\prime}
\end{array}\right)_{L}, R_{c}=c_{R}, R_{s}=s_{R}
\end{aligned}
$$




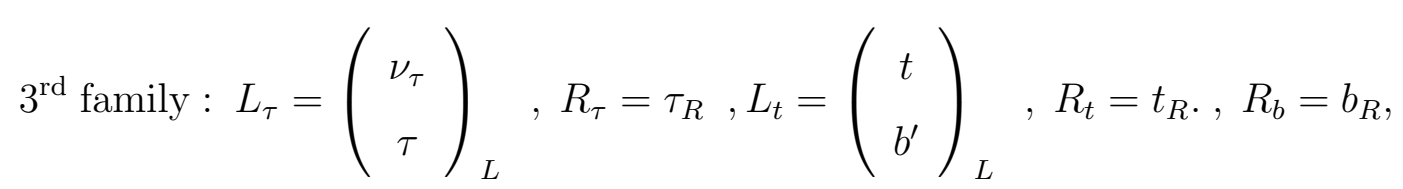

$L(R)$ stands for left (right) handed fermion: if $\Psi$ represents the Dirac spinor of the fermion, $\Psi_{L}=\frac{1-\gamma^{5}}{2} \Psi\left(\Psi_{R}=\frac{1+\gamma^{5}}{2} \Psi\right)$ where $\gamma^{5}$ is the Dirac matrix defined in [4]. In the quark families, the $d_{i}^{\prime}=\left(d^{\prime}, s^{\prime}, b^{\prime}\right)$ and the $d_{i}=(d, s, b)$ are related by the Cabibbo-Kobayashi-Maskawa matrix [5] [6]. The free Lagrangian is built from kinetic contributions expressed as $\mathcal{L}=\Psi^{\dagger} i \partial_{\mu} \gamma^{\mu} \Psi$. Once all free matter fields are taken into account, we impose the local gauge invariance under a unitary group denoted $U(1)_{Y} \times S U(2)_{L} \times S U(3)_{C}$, where $Y$ is the hyper-charge, $L$ the handedness and $C$ the color. To achieve this in the case of the $U(1)_{Y}$ and $S U(2)$ groups we must replace the derivative of the fields with the covariant derivative:

$$
D_{\mu}=\partial_{\mu}-i g \tau \cdot W_{\mu}-i g^{\prime} Y B_{\mu}
$$

which involves the appropriate electromagnetic and weak charges, $g$ and $g^{\prime}[7]$. By construction, the Lagrangian now exhibits fermion-gauge boson interaction terms. Three of the bosons denoted $W_{\mu}^{(1)}, W_{\mu}^{(2)}$ and $W_{\mu}^{(3)}$ are the components of a triplet of the group $S U(2)$ representing the weak interaction. A fourth boson, $B_{\mu}$, belongs to the $U(1)_{Y}$ group of the weak hyper-charge. The SM includes also the $S U(3)_{C}$ group associated to eight bosons, the gluons $g$, carrying the strong interaction. If the symmetry is exact, the associated gauge boson are massless. However, measurements at the UA1, UA2, LEP and Tevatron experiments indicated that the gauge bosons associated to the weak interaction do have mass. The direct introduction of boson mass terms in the Lagrangian would ruin the gauge invariance of the SM therefore the theory has to be modified to include a symmetry breaking mechanism which gives origin to mass. This is achieved by means of a doublet of complex fields called the Higgs field [8]:

$$
\phi=\left(\begin{array}{c}
\varphi^{+} \\
\varphi^{0}
\end{array}\right)
$$


The corresponding Lagrangian is invariant under $S U(2)_{L} \times U(1)_{Y}$ if the Higgs potential is defined:

$$
V=-\mu^{2} \phi^{\dagger} \phi+\lambda\left(\phi^{\dagger} \phi\right)^{2}
$$

where $\lambda$ represents the quartic coupling term. Since the potential in Figure 2.1 is minimized by $\phi^{\dagger} \phi=\frac{\mu^{2}}{2 \lambda}$, the field $\phi$ can be expanded around a particular ground state with:

$$
<\phi>=\frac{1}{\sqrt{2}}\left(\begin{array}{l}
0 \\
v
\end{array}\right)
$$

where

$$
v=\sqrt{\frac{\mu^{2}}{\lambda}}
$$

inducing the symmetry breaking. The fluctuation around the ground state is the



Figure 2.1. Schematic view of the Higgs potential with $\mu^{2}<0$ and $\lambda>0$.

physical Higgs with mass $m_{h}=2 \mu^{2}=2 \lambda v^{2}$. The mass terms for the gauge bosons originate from the kinetic term of $\phi$. The physical bosons are now denoted $W_{\mu}^{+}, W_{\mu}^{-}$, $Z_{\mu}^{0}$ and the photon $A_{\mu}$. The neutral bosons $A_{\mu}$ and $Z_{\mu}^{0}$ are combinations of the $W_{\mu}^{(3)}$ 
and $B_{\mu}$, while the the charged ones $W_{\mu}^{+}$and $W_{\mu}^{-}$are a mixture of the $W_{\mu}^{(1)}, W_{\mu}^{(2)}$. The $S U(2)$ symmetry is broken and the weak bosons acquire mass:

$$
m_{W}=g \frac{v}{2} ; \quad m_{Z}=\sqrt{g^{2}+g^{\prime 2}} \frac{v}{2}
$$

while the photon $A_{\mu}$, carrier of the electromagnetic interaction, remains massless since the $U(1)_{E M}$ is preserved. The mass scale $v=246 \mathrm{GeV}$ is determined from the measured mass of the $W$ and $Z$ bosons.

Based on the same mechanism it is also possible to construct mass terms for the fermion fields as well. In the limit of exact $S U(2) \times U(1)$ symmetry, a term which mixes left and right handed fermion to provide mass is forbidden ${ }^{1}$; however the symmetry is broken by the Higgs mechanism and the Higgs can couple right to left handed fermions ${ }^{2}$. These mass terms are denoted Yukawa couplings. The fundamental fermions and gauge bosons in the Standard Model are given in the Table 2.1.

\subsection{Open questions in the SM}

The HEP experiments have explored an energy range up to hundreds of GeV and the SM has been proven to be extremely successfully since no significant deviations from its predictions have been observed. Currently, the Tevatron collider is searching for the last ingredient, the Higgs boson, which has escaped detection so far. Nevertheless, the SM is regarded as the low energy effective model of a more fundamental theory since there are several unresolved issues.

The SM does not include the gravitational interaction which becomes non-negligible at the so called Planck scale. As a consequence the SM can not be extended up to a scale of $10^{18} \mathrm{GeV}$. Furthermore, why is the EWK scale, fixed by the Higgs field vacuum expectation value, so much lower than the Planck scale? This is known as the "hierarchy problem", tightly related to the "naturalness" problem: we expect the

${ }^{1}$ For example the left handed electron carries isospin $I=\frac{1}{2}$ and hyper-charge $Y=-\frac{1}{2}$ while the right handed electron isospin $I=0$ and hyper-charge $Y=-1$.

${ }^{2}$ The Higgs carries isospin $I=\frac{1}{2}$ and hyper-charge $Y=+\frac{1}{2}$. 
Table 2.1

List of the Standard Model particles. The left handed leptons and quarks are weak-isospin $\left(S U(2)_{L}\right)$ doublets. The right handed leptons and quarks are weak-isospin $\left(S U(2)_{L}\right)$ singlets. Each quark is a color $\left(S U(3)_{C}\right)$ triplet. All other particles are color $\left(S U(3)_{C}\right)$ singlets.)

\begin{tabular}{|c|c|c|c|}
\hline Particle & Spin & Electric charge & Hyper-charge \\
\hline \hline$\nu_{e}$ & $\frac{1}{2}$ & 0 & $\left(\nu_{e}\right)_{L}=-\frac{1}{2}$ \\
$e$ & $\frac{1}{2}$ & -1 & $e_{L}=-\frac{1}{2} ; e_{R}=-1$ \\
$\nu_{\mu}$ & $\frac{1}{2}$ & 0 & $\left(\nu_{\mu}\right)_{L}=-\frac{1}{2}$ \\
$\mu$ & $\frac{1}{2}$ & -1 & $\mu_{L}=-\frac{1}{2} ; \mu_{R}=-1$ \\
$\nu_{\tau}$ & $\frac{1}{2}$ & 0 & $\left(\nu_{\tau}\right)_{L}=-\frac{1}{2}$ \\
$\tau$ & $\frac{1}{2}$ & -1 & $\tau_{L}=-\frac{1}{2} ; \tau_{R}=-1$ \\
\hline \hline$u$ & $\frac{1}{2}$ & $\frac{2}{3}$ & $u_{L}=+\frac{1}{6} ; u_{R}=+\frac{2}{3}$ \\
$d$ & $\frac{1}{2}$ & $-\frac{1}{3}$ & $d_{L}=+\frac{1}{6} ; d_{R}=-\frac{1}{3}$ \\
$c$ & $\frac{1}{2}$ & $\frac{2}{3}$ & $c_{L}=+\frac{1}{6} ; c_{R}=+\frac{2}{3}$ \\
$s$ & $\frac{1}{2}$ & $-\frac{1}{3}$ & $s_{L}=+\frac{1}{6} ; s_{R}=-\frac{1}{3}$ \\
$t$ & $\frac{1}{2}$ & $\frac{2}{3}$ & $t_{L}=+\frac{1}{6} ; t_{R}=+\frac{2}{3}$ \\
$b$ & $\frac{1}{2}$ & $-\frac{1}{3}$ & $b_{L}=+\frac{1}{6} ; b_{R}=-\frac{1}{3}$ \\
\hline$Z^{0}$ & 1 & 0 & 0 \\
$W^{+}$ & 1 & -1 & +1 \\
\hline \hline & 1 & -1 & 0 \\
\hline \hline
\end{tabular}

Higgs mass of the order of hundreds of $\mathrm{GeV} / c^{2}$ therefore the radiative corrections to the its mass become of several orders of magnitude (Figure 2.2). The Higgs sector is also correlated to the running of the coupling constants. If we extrapolate the experimental values to large energies, we expect the unification of the gauge interactions, which is not achieved in the Standard Model. Finally, the most stunning open 


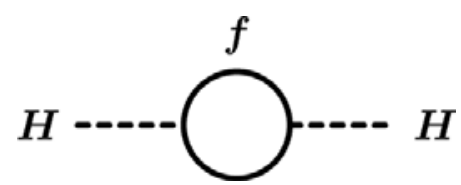

Figure 2.2. Correction to $\mathrm{m}_{H}^{2}$ from a loop containing a Dirac fermion $f$ with mass $m_{f} . \Lambda_{U V}$ is the ultraviolet momentum.

question comes from the Universe since the SM is able to explain just a tiny fraction $(\sim 4 \%)$ of it. 


\section{Supersymmetry}

Supersymmetry (SUSY) is a proposed symmetry of Nature. It is defined such that for each SM particle, exists a new Supersymmetric partner with the same quantum

numbers but the spin. With the introduction of SUSY, the quadratic divergences of the Higgs mass naturally cancel. SUSY can also provide an excellent candidate for the Dark Matter in the Universe. In what follows, we briefly introduce the SUSY formalism. Finally we discuss the expected SUSY spectrum.

For the details about the theoretical framework, refer to [9], [10], [11], [12] and [13].

\subsection{SUSY Formalism}

Since SUSY is a symmetry which relates fermions to bosons, we first define a convenient representation of fermions. Based on this we provide a simplified picture of its formalism by taking the following steps:

- connect fermions to bosons through a symmetry,

- understand the particle content,

- build the SUSY Lagrangian.

\subsubsection{Fermions}

The Dirac Lagrangian for a massive field can be written as [14]

$$
\mathcal{L}=\bar{\psi} i \not \partial \psi-m \bar{\psi} \psi
$$


where $\psi$ is a 4 component spinor. The Dirac representation is reducible and we can form a $2 \mathrm{D}$ representation writing the spinor in terms of left and right handed complex fields $\left(\psi_{L}, \psi_{R}\right)$. The free Lagrangian becomes,

$$
\mathcal{L}=\psi_{L}^{\dagger} i \bar{\sigma}^{\mu} \partial_{\mu} \psi_{L}+\psi_{R}^{\dagger} i \bar{\sigma}^{\mu} \partial_{\mu} \psi_{R}-m\left(\psi_{R}^{\dagger} \psi_{L}+\psi_{L}^{\dagger} \psi_{R}\right)
$$

In Equation 3.2 the left and right handed fields couple in the mass term only. In order to simplify the notation, we benefit from the feature that the antiparticle of a massless left handed Dirac particle is right handed, and write all left handed states as particles and all right handed ones as the antiparticle of a left handed particle [15]. To do so we define,

$$
\chi_{L}=c \psi_{R}^{*} ; \quad \chi_{L}^{*}=c \psi_{R} \quad c=\left(\begin{array}{cc}
0 & -1 \\
1 & 0
\end{array}\right)
$$

The kinetic terms do not change their form, but the mass term becomes symmetric under the exchange of the two fields $(\psi$ and $\chi)$,

$$
-m\left(\chi_{L}^{T} c \psi_{L}-\psi_{L}^{\dagger} c \chi_{L}^{*}\right)
$$

We can infer a general mass term for fermions of the form

$$
-\frac{1}{2} m^{a b} \psi_{L}^{a T} c \psi_{L}^{b}+\text { h.c. }
$$

$m^{a b}$ being a symmetric matrix. From now on, we use a notation where each fermion is a 2 component left handed field and we discard the subscript $\mathrm{L}$.

\subsubsection{From fermions to bosons}

We start from the free Lagrangian of a massless fermion and a massless boson field:

$$
\mathcal{L}=\partial_{\mu} \phi^{*} \partial^{\mu} \phi+\psi^{\dagger} i \sigma \cdot \partial \psi
$$

and we look for a symmetry which could link fermions and bosons, whose parameter should therefore carry half integer spin. A possibility is to define a left handed chiral spinor $\xi$ such that

$$
\delta_{\xi} \phi=\sqrt{2} \xi^{T} c \psi
$$




$$
\delta_{\xi} \psi=\sqrt{2} i \sigma \cdot \partial \phi c \xi^{*}
$$

are symmetries of the Lagrangian. If we interpret the transformation $\delta_{\xi}$ as the action of a set of operators Q called the supersymmetry charges

$$
\delta_{\xi}=\xi^{T} c Q-Q^{\dagger} c \xi^{*}
$$

we find the following algebra for supersymmetry:

$$
\left\{Q_{a}^{\dagger}, Q_{b}\right\}=\left(\bar{\sigma}^{\mu}\right)_{a b} P_{\mu}
$$

where $P_{\mu}$ is the energy-momentum 4-vector. This result proves that each non zero energy state has a partner with different spin. In fact, if we consider the case $a=$ $b=1$, Equation 3.10 transforms into

$$
\left\{Q_{1}^{\dagger}, Q_{1}\right\}=P^{0}+P^{3}=P^{+}
$$

If we build $a=\frac{Q_{1}}{\sqrt{P^{+}}}$and $a^{\dagger}=\frac{Q_{1}^{\dagger}}{\sqrt{P^{+}}}$, the $a$ and $a^{\dagger}$ satisfy $\left\{a^{\dagger}, a\right\}=1$, which is the algebra of fermion raising and lowering operators. This proves that the generators of Supersymmetry, Q, link fermions and bosons $\left(\Delta J^{3}= \pm \frac{1}{2}\right)$.

\subsubsection{Particle content of the MSSM}

In a supersymmetric theory all particles fall into a representation called supermultiplet, similarly to the proton and the neutron belonging to a doublet for the isospin. Each supermultiplet contains the SM particle and its supersymmetric partner, found by applying a combination of $Q$ and $\bar{Q}$ to the original state.

The first type of superfield is the chiral supermultiplet (also called matter superfield), which contains a left handed fermion, its right handed antiparticle, a complex boson and its conjugate. For instance the partner of the electron $\left(e_{L}^{-}\right)$is the selectron $\left(\tilde{e}_{L}^{-}\right)$ while the partner of the right handed electron is another selectron $\left(\tilde{e}_{R}^{-}\right)$. The Higgs boson also belongs to a chiral superfield, paired to a spin $\frac{1}{2}$ fermion denoted Higgsino. In particular, the Higgs sector contains two doublets and we will see the reason why 
later. In the gauge sector, there is a vector supermultiplet for each gauge boson, containing two vector bosons with different polarization and a left handed fermion $\lambda^{a}$ with its antiparticle: eight gluinos $\tilde{g}$ as partners of the QCD gluons, three winos $\tilde{W}$ for the $S U(2)_{L}$ gauge bosons, a bino $\tilde{B}$ for $U(1)_{Y}$. The supersymmetric particles are called gauginos.

The details of the notation are summarized in Table 3.1 and Table 3.2.

Table 3.1

Chiral multiplets in the MSSM.

\begin{tabular}{|c|c|c|c|}
\hline multiplet & & spin 0 & spin 1/2 \\
\hline \hline squarks, quarks & $Q$ & $\left(\tilde{u}_{L}, \tilde{d}_{L}\right)$ & $\left(u_{L}, d_{L}\right)$ \\
squarks, quarks & $\bar{U}$ & $\tilde{u}_{R}^{*}$ & $\bar{u}_{R}$ \\
squarks, quarks & $\bar{D}$ & $\tilde{d}_{R}^{*}$ & $\bar{d}_{R}$ \\
sleptons, leptons & $L$ & $\left(\tilde{\nu}_{L}, \tilde{e}_{L}\right)$ & $\left(\nu_{L}, e_{L}\right)$ \\
sleptons, leptons & $\bar{E}$ & $\tilde{e}_{R}^{*}$ & $\bar{e}_{R}$ \\
Higgs, Higgsino & $H_{1}$ & $\left(H_{1}^{+}, H_{1}^{0}\right)$ & $\left(\tilde{H}_{1}^{+}, \tilde{H}_{1}^{0}\right)$ \\
Higgs, Higgsino & $H_{2}$ & $\left(H_{2}^{0}, H_{2}^{-}\right)$ & $\left(\tilde{H}_{2}^{0}, \tilde{H}_{2}^{-}\right)$ \\
\hline
\end{tabular}

Table 3.2

Vector multiplets in the MSSM.

\begin{tabular}{|c|c|c|}
\hline multiplet & spin 1/2 & spin 1 \\
\hline \hline gluino, gluons & $\tilde{g}$ & $g$ \\
winos, W bosons & $\tilde{W}^{ \pm} \tilde{W}^{0}$ & $W^{ \pm} W^{0}$ \\
binos, B bosons & $\tilde{B}^{0}$ & $B^{0}$ \\
\hline
\end{tabular}




\subsubsection{SUSY Lagrangian}

To build the Lagrangian for the chiral supermultiplets, we consider Equation 3.6, and introduce a so called auxiliary field $F^{1}$ as follows:

$$
\mathcal{L}=\partial_{\mu} \phi^{*} \partial^{\mu} \phi+\psi^{\dagger} i \bar{\sigma} \cdot \partial \psi+F^{\dagger} F+m\left(\phi F-\frac{1}{2} \psi^{T} c \psi\right)+h . c .
$$

$\mathrm{F}$ is a complex field satisfying $F^{\dagger}=-m \phi$ and $F=-m \phi^{*}$, and it can be integrated out using the equation of motion. If these expression are inserted back into Equation 3.12, the Lagrangian becomes:

$$
\mathcal{L}=\partial_{\mu} \phi^{*} \partial^{\mu} \phi-m \phi^{*} \phi+\psi^{\dagger} i \bar{\sigma} \cdot \partial \psi-\frac{1}{2} m\left(\psi^{T} c \psi-\psi^{\dagger} c \psi^{*}\right)
$$

where particles and superpartners are now massive (with a degenerate mass $m$ ). To interpret the mass term we interpret the Lagrangian in Equation 3.12 as a special case of a more general supersymmetric Lagrangian

$$
\mathcal{L}=\partial_{\mu} \phi_{j}^{*} \partial^{\mu} \phi_{j}+\psi^{\dagger} i \bar{\sigma} \cdot \partial \psi_{j}+F^{\dagger} F_{j}+\left(F_{j} \frac{\partial W}{\partial \phi_{j}}-\frac{1}{2} \psi_{j}^{T} c \psi_{k} \frac{\partial^{2} W}{\partial_{j} \phi \partial_{k} \phi}\right)+\text { h.c. }
$$

where $F^{\dagger}=-\frac{\partial W}{\partial \phi_{j}}$ ( $j$ indicates the chiral supermultiplet). W is a function of the field $\phi$ only and it does not depend on $\phi^{\dagger}$. The Lagrangian of massive particles in Equation 3.12 is the result of the choice $W=\frac{1}{2} m \phi^{2}$. If

$$
W=\lambda_{u}^{i j} \bar{u}^{i} h_{2} \cdot Q^{j}+\lambda_{d}^{i j} \bar{d}^{i} h_{1} \cdot Q^{j}+\lambda_{l}^{i j} \bar{e}^{i} h_{1} \cdot L^{j}
$$

this superpotential can be interpreted as the generalization of the SM Yukawa terms. Thus, in a supersymmetric theory, the Yukawa interactions come from a superpotential that cannot depend on a field and its conjugate at the same time (on contrast to the SM). As a result, a doublet can give mass either to $T_{3}=+\frac{1}{2}$ or to $T_{3}=-\frac{1}{2}$. In order to give mass to all sfermions, two Higgs doublets must instead be introduced. As anticipated, we want to introduce the vector supermultiplets $\left(A_{\mu}^{a}, \lambda^{a}\right)$ and their coupling to the chiral superfields. The Lagrangian can be written as,

$$
\mathcal{L}=-\frac{1}{4}\left(F_{\mu \nu}^{a}\right)^{2}+\lambda^{\dagger, a} i \bar{\sigma}^{\mu} D_{\mu} \lambda_{\frac{1}{2}}^{a}\left(D^{a}\right)^{2}
$$

${ }^{1}$ The auxiliary field $F$ is introduced to allow the symmetry to close off-shell: the symmetry holds quantum mechanically. 
where, similarly to Equation 3.12, we introduce a new auxiliary field $D^{a}$ which satisfies $D^{a}=-g \sum_{j} \phi^{\dagger} t^{a} \phi$ where $t^{a}$ is the gauge group generator. In addition to the kinetic energy for the SM gauge fields, Equation 3.16 contains the kinetic terms of the gauginos and the coupling of gauge bosons to gauginos. Since the vector supermultiplets contain gauge fields, chiral supermultiplets that transform non trivially under the gauge group should couple to them to keep the total Lagrangian invariant. The Lagrangian in Equation 3.14 will be modified accordingly.

Finally we can combine the different pieces into a globally supersymmetric Lagrangian,

$$
\mathcal{L}=\mathcal{L}_{\text {chiral }}+\mathcal{L}_{\text {Yukawa }}+\mathcal{L}_{\text {gauge }}+\mathcal{L}_{\mu}
$$

where

- $\mathcal{L}_{\text {chiral }}$ includes the kinetic energy and gauge interaction terms of quarks, leptons and Higgs chiral supermultiplets;

- $\mathcal{L}_{\text {Yukawa }}$ contains the Yukawa and scalar interactions of Equation 3.15;

- $\mathcal{L}_{\text {gauge }}$ is the kinetic energy of the vector supermultiplets.

The SUSY Lagrangian conserves a new quantum number called R-parity and defined $R_{P}=(-1)^{3 B+2 S-L}$. This new conserved quantum number leads to crucial experimental implications as described in Chapter 4. SM particles and superparticles have $R_{P}=1$ and $R_{P}=-1$ respectively. Yukawa terms with an odd number of superparticles are forbidden.

\subsubsection{SUSY breaking}

The supersymmetric Lagrangian is such that the Standard Model particles have the same mass as their supersymmetric partners. This is clearly not realistic since, for example, no supersymmetric electron with a mass of $511 \mathrm{MeV} / \mathrm{c}^{2}$ has been observed. Searches conducted at the LEP $e^{+} e^{-}$collider showed that charginos and charged sleptons must be heavier than $\sim 100 \mathrm{GeV} / c^{2}[16]$. The Tevatron experiments have set 
bounds on the mass of squark and gluinos to be larger than $250 \mathrm{GeV} / c^{2}[16]$. Hence SUSY must be a broken symmetry. The underlying idea is that SUSY is spontaneously broken in a new sector of particles at high energy ("hidden sector"). The coupling between the supermultiplets and the new particles induce the breaking of the symmetry, and it is associated to a mass scale $\mathcal{M}$ (the messenger fields between the hidden sector and the supermultiplets). In the low energy effective theory, the breaking mechanism is parametrized by inserting so called soft terms into the Lagrangian. "Soft" indicates that the cancellation of the quadratic divergences is still preserved, since it does not rely on the SUSY and SM particles having the same mass but having the same coupling. Possible soft breaking terms will have the form:

- $-m_{\phi_{i}}^{2}\left|\phi^{2}\right|$, mass term for the scalar member of the chiral multiplets;

- $-\frac{1}{2} M_{a} \bar{\lambda}_{a} \lambda_{a}$, gauginos mass term where a labels the group; $\left(M_{1}, M_{2}, M_{3}\right.$ for, respectively, $\left.U(1)_{Y} \times S U(2)_{L} \times S U(3)_{C}\right)$;

- $-A_{i j k} \phi_{i} \phi_{j} \phi_{k}+$ h.c, trilinear scalar interactions;

- $\mu h_{1} \cdot h_{2}$, Higgsino mass term.

In particular if the $\tilde{t}$ is heavier than the top quark, the mass of the Higgs $h_{1}$ becomes negative at the EWK scale and the breaking of the EWK symmetry occurs naturally.

\subsubsection{Mass spectrum in the context of the Minimal Supersymmetric Stan- dard Model (MSSM)}

If SUSY is exact, the SM particles are prevented from acquiring mass thanks to the gauge symmetries of the SM. However SUSY is a broken symmetry and the new particles can acquire large masses. At the GUT scale the masses of the supersymmetric particles differ from their SM partners if the scale of the messenger is above the GUT scale itself. In this scenario, we can unify all gauginos into a universal mass $m_{\frac{1}{2}}$; for sleptons and squarks, the universality requires a common mass denoted $m_{0}$. 
The Renormalization Group Equations (RGE) are used to evolve the mass from the GUT to the EWK scale and yield the following relations between the masses and the couplings [17]:

$$
\frac{m_{i}(Q)}{\alpha_{i}(Q)}=\frac{m_{\frac{1}{2}}}{\alpha_{U}} \quad m_{1}=0.5 \times m_{2} \quad m_{3}=3.5 \times m_{2} .
$$

In the Higgs sector, the original eight degrees of freedom from the four real scalar fields of each doublet reduce to five physical scalar particles after the symmetry breaking. There are two CP-even scalars $h^{0}, H^{0}$, one CP-odd scalar $A^{0}$, and two charged scalars $H^{+}$and $H^{-}$with the following mass relations:

$$
\begin{gathered}
m_{A}^{2}=2 \mu^{2}+m_{H_{u}}^{2}+m_{H_{d}}^{2}, \quad m_{H^{ \pm}}^{2}=m_{W}^{2}+m_{A}^{2}, \\
m_{h^{0}}^{2}, m_{H^{0}}^{2}=\frac{1}{2}\left(m_{A}^{2}+m_{Z}^{2} \pm \sqrt{\left(m_{A}^{2}+m_{Z}^{2}\right)^{2}-4 m_{Z}^{2} m_{A}^{2} \cos ^{2} 2 \beta}\right) .
\end{gathered}
$$

The lighter CP-even Higgs mass $m_{h^{0}}^{2}$ becomes large when $\cos ^{2} 2 \beta=1$, where $\beta$ is defined as the ratio of the two expectation values of the two Higgs doublets $\tan \beta=$ $\frac{<v_{1}>}{<v_{2}>}$. When $m_{A}<m_{Z}, m_{h^{0}}^{2}=m_{A}^{2}<m_{Z}^{2}$, otherwise $m_{h^{0}}^{2}=m_{Z}^{2}$, leading to:

$$
m_{h^{0}} \leq m_{Z}
$$

These predictions are modified by the quantum corrections to the mass, alias:

$$
\Delta\left(m_{h^{0}}^{2}\right) \sim v^{2} \sin ^{4} \beta \log \left(\frac{m_{\tilde{t}_{1}} m_{\tilde{t}_{2}}}{m_{t}^{2}}\right)
$$

where $\tilde{t}_{1}$ and $\tilde{t}_{2}$ are the mass eigenstates of the stop, the scalar partner of the SM top quark. If their mass is fixed to the scale $1 \mathrm{TeV} / c^{2}$ and the top mass is $175 \mathrm{GeV} / c^{2}$, then the lightest Higgs mass is lighter than $130 \mathrm{GeV} / c^{2}$. This is a crucial prediction of the MSSM.

Similarly, the sfermions are subject to corrections which can reduce the value of the mass, in particular, of the third generation. The third generation undergoes also a significant mass splitting due to the large Yukawa coupling to the Higgs sector. 


\section{Charginos and Neutralinos}

The gluinos, being a color octet, are unmixed with an expected mass $m_{\tilde{g}}=\left|M_{3}\right|$. The electroweak gauginos and higgsinos mix, similarly to the gauge bosons and the Goldstone modes after the spontaneous symmetry breaking. The charginos mass states $\left(\tilde{\chi}_{i}^{ \pm}\right)$are mixtures of the electrically charged $S U(2)_{L}$ gauginos and the electrically charged higgsinos. Their mass matrix in the $\left(\tilde{W}^{ \pm}, H^{ \pm}\right)$basis can be written as:

$$
\mathcal{M}_{ \pm}=\left(\begin{array}{cc}
M_{2} & \sqrt{2} M_{W} \sin \beta \\
\sqrt{2} M_{W} \cos \beta & \mu
\end{array}\right) .
$$

where $\mu$ is the higgsino mixing term and $M_{2}$ the $S U(2)$ gaugino mass.

The neutralinos $\left(\tilde{\chi}_{i}^{0}\right)$ are mixtures of the $\tilde{B}$, the neutral $\tilde{W}$, and the two neutral higgsinos. These states form four distinct Majorana fermions, which are eigenstates of a mass matrix in the $\left(\tilde{B}, \tilde{W}, \tilde{h}^{0}, \tilde{\bar{h}}^{0}\right)$ basis:

$$
\mathcal{M}_{0}=\left(\begin{array}{cccc}
M_{1} & 0 & -M_{Z} \cos \beta \sin \theta_{W} & M_{Z} \sin \beta \sin \theta_{W} \\
0 & M_{2} & M_{Z} \cos \beta \cos \theta_{W} & -M_{Z} \sin \beta \cos \theta_{W} \\
-M_{Z} \cos \beta \sin \theta_{W} & M_{Z} \cos \beta \cos \theta_{W} & 0 & -\mu \\
M_{Z} \sin \beta \sin \theta_{W} & -M_{Z} \sin \beta \cos \theta_{W} & -\mu & 0
\end{array}\right)
$$

The masses and mixing angles of the charginos and neutralinos are therefore completely determined by the values of the $M_{1}, M_{2}, \mu, \tan \beta$ and $\sin$ of the weak mixing angle $\theta_{W}$.

- if $|\mu|>\left|M_{2}\right|>>m_{W}, \tilde{\chi}_{1}^{ \pm}$is mainly a $\tilde{W}^{ \pm}$with mass $\sim M_{2}$; the next to lightest chargino is dominated by the $h_{2}$ component yielding a mass mass $\sim \mu$; the lightest neutralino is mostly a bino with mass $\sim M_{1}$, while $\tilde{\chi}_{2}^{0}$ is mainly $\tilde{W}^{3}$ with mass $\sim M_{2}$;

- if $\left|M_{2}\right|>|\mu|>>m_{W}$, the lightest chargino and neutralino are mainly higgsino like;

- if $\left|M_{2}\right|=|\mu|$, there is a non negligible mixing between the fields, in particular for heavy charginos and neutralinos at large $\tan \beta$. 
The first regime is called "gaugino" region, the second "higgsino" region. In the gaugino region, the mass relation can be simplified to,

$$
m_{\tilde{\chi}_{i}^{ \pm}} \simeq m_{\tilde{\chi}_{2}^{0}} \simeq 2 m_{\tilde{\chi}_{1}^{0}}
$$

Depending on the higgsino and gaugino components, the coupling to the gauge bosons and to the left and right handed sfermions differ radically. In the "gaugino" region the couplings are governed by the gauge couplings, while in the "higgsino" region by the Yukawas ones.

\subsection{Minimal supergravity}

As explained in Section 3.1.5, the symmetry breaking is parametrized by adding the soft terms to the low energy effective Lagrangian. Nevertheless, there are theoretical models proposed to explain how SUSY breaks. The most common model is called minimal supergravity or mSUGRA [18]. In mSUGRA the superfields in the hidden sector acquire a vacuum expectation value $<F>$ through a Higgs mechanism and interact with the supermultiplets through gravity. As a consequence the mass scale of the soft terms is proportional to $\frac{1}{M_{\text {Planck }}}$. The number of free parameters can be reduced by assuming universality of the coupling between the supermultiplets and the hidden sector; as in the MSSM, the scalars (gauginos) bear a common mass denoted mass $m_{0}\left(m_{\frac{1}{2}}\right)$. In mSUGRA the trilinear couplings are unified at the GUT scale to the value $A_{0}$. The additional two free parameters of the model are the ratio of the Higgs doublets expectation values, $\tan (\beta)$, and the sign of the Higgs mixing term, $\operatorname{sgn}(\mu)$. In the mSUGRA scenario the higgsino term $\mu$ is typically greater than the bino and wino mass; as a result the lightest neutralino and chargino tend to be gaugino-like. The squark and slepton mass parameters for the first and second generation can be parametrized as:

$$
m_{\tilde{q}_{L}}^{2} \sim m_{0}^{2}+6 \cdot m_{\frac{1}{2}}^{2} ; \quad m_{\tilde{q}_{R}}^{2} \sim m_{0}^{2}+5 \cdot m_{\frac{1}{2}}^{2}
$$


whereas slepton masses satisfy:

$$
m_{\tilde{\ell}_{L}}^{2} \sim m_{0}^{2}+0.5 \cdot m_{\frac{1}{2}}^{2} ; \quad m_{\tilde{\ell}_{R}}^{2} \sim m_{0}^{2}+0.15 \cdot m_{\frac{1}{2}}^{2}
$$

The spectra has the following features. The squarks are heavier than the sleptons because they interact through the strong and EWK couplings. The right-handed partners are lighter than left-handed ones since they carry only the hyper-change quantum number. In the third generation, the Yukawa interactions tend to reduce the scalar mass so that $\tilde{t}_{L}$ and $\tilde{t}_{R}$ tend to be lighter than the other squarks; the Yukawa terms also induce mixing between left and right squarks further reducing the mass of the lightest stop $\left(\tilde{t}_{1}\right)$. We expect a similar trend for $\tilde{\tau}$ s.

\subsection{Does SUSY answer the open questions in the SM?}

The major achievement of SUSY is the cancellation of the radiative corrections of the Higgs mass. For a particular choice of superpotential, the vertices from the Yukawa terms are of the same order of magnitude yielding a cancellation of the scalar field mass corrections. In addition, the coupling constants extrapolated from the experimental values to high energies, unify at the GUT scale, while the unification is not achieved in the SM as shown in Figure 3.1. Finally if R-parity is not violated, the lightest supersymmetric particle, the LSP, is neutral and stable representing an excellent Dark Matter candidate. 

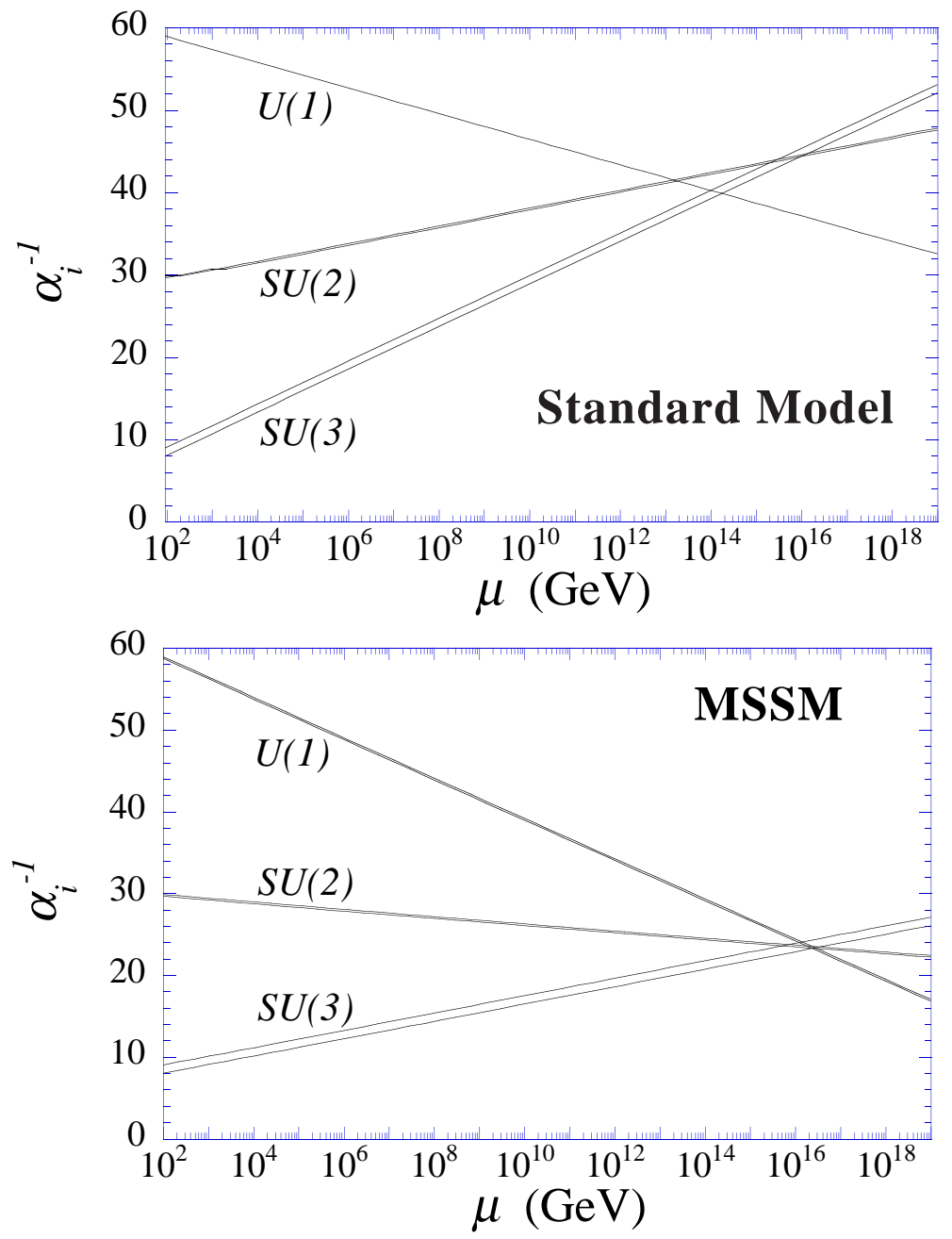

Figure 3.1. Running of gauge coupling constants in the SM and in the MSSM frameworks as a function of the energy scale denoted $\mu$ [9]. 


\section{Sensitivity to SUSY at the Run II of the Tevatron}

In the mSUGRA scenario, the symmetry is broken via gravitational interactions and originates a rich spectrum of particles at the EWK scale, as shown in Figure 4.1 [15]. Note that the partners of the $\mathrm{W}, \mathrm{B}$ and $\mathrm{H}$ bosons are the lightest supersymmetric

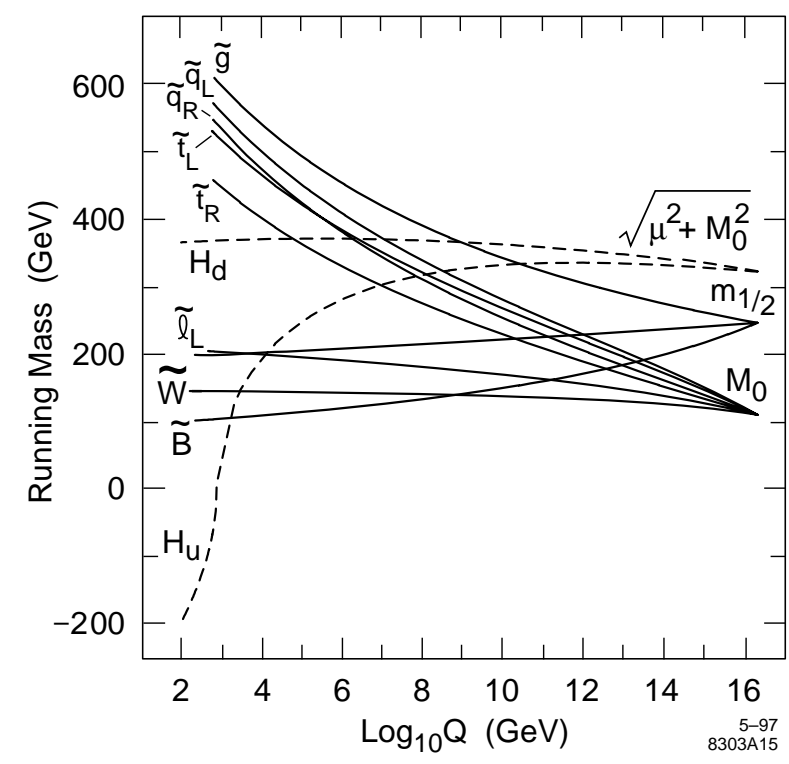

Figure 4.1. Evolution of the soft supersymmetry breaking mass terms in the Universal MSSM.

particles and mix into mass eigenstates called charginos and neutralinos as described in Section 3.1.6. As a consequence, their associated production exhibits one of the largest SUSY cross sections at the Tevatron as shown in Figure 4.2 where the production cross sections of various SUSY processes are compared as a function of the SUSY particle mass [19]. It should be noted that besides the production of the supersymmetric particles, SUSY may also reveal itself via indirect effects, such as in contributions to decays expected to be rare in the SM. Furthermore, a relic SUSY cold dark matter might have been already detected in cosmological experiments [20]. 


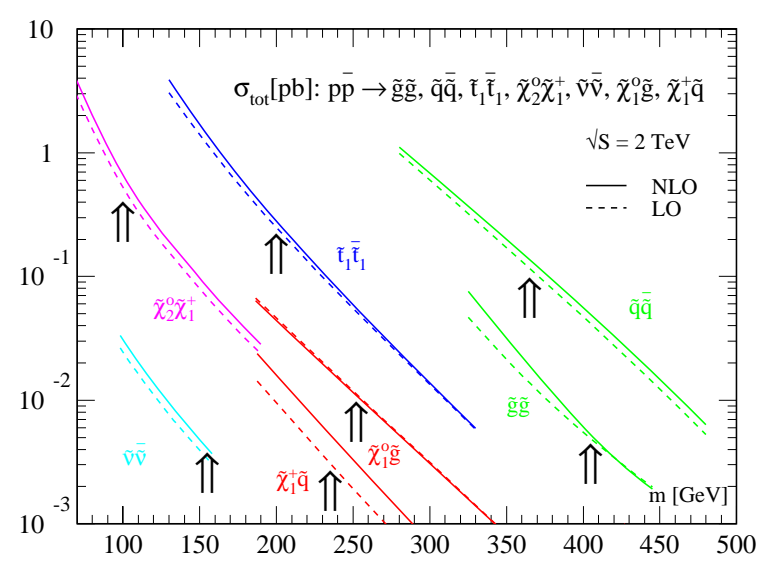

Figure 4.2. SUSY production cross section as a function of the superparticle mass.

Before describing the direct search for chargino-neutralino production we perform at Tevatron, we summarize the constraints of indirect searches to the mSUGRA space.

\subsection{Current constraints in mSUGRA}

\subsubsection{Cold dark matter}

One of the best evidence of New Physics is the measured density of Dark Matter in the Universe from the WMAP survey [20]. If the DM is interpreted in terms of thermal relic density of weakly interacting massive particles (WIMPs), it is denoted Cold Dark Matter, or CDM. The expected mass of the WIMP is subject to tight constraints, since its mass and its annihilation rate are required to match the measured DM density; moreover, the new particle has to be the lightest particle of the theory to be stable. In mSUGRA the candidate for DM is typically the lightest neutralino $\tilde{\chi}_{1}^{0}$; its density, expressed in terms of the annihilation cross section $\sigma_{a n n}$ and the the relative velocity between two neutralinos $v[21]$

$$
\Omega_{\tilde{\chi}_{1}^{0}} \sim \int d x\left(<\sigma_{\text {ann }} \times v>\right)^{-1}
$$


is larger than the value measured by WMAP in most of the parameter space [22]. However there are regions where the neutralino density lies in the observed range accounting for the whole dark matter in the Universe $^{1}$, in particular:

- The bulk region, where both $m_{\frac{1}{2}}$ and $m_{0}$ are small (Figure 4.9) and the neutralino annihilates in the t-channel through exchange of light sleptons. This region is tightly constrained since the LSP is almost a pure bino and the annihilation of binos in fermion pairs is to small. In order to enhance the rate, the contribution of the annihilation via Higgs states must be included. However such contribution becomes large only for high $\tan \beta$ (see below).

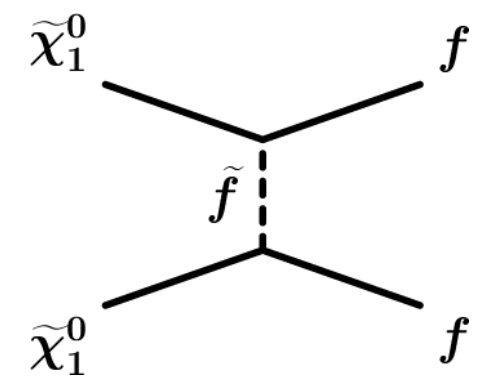

Figure 4.3. Neutralinos annihilation via slepton.

- The Higgs annihilation funnel (Figure 4.4) includes both a light Higgs $(h)$ channel at low $m_{\frac{1}{2}}$ and a heavy Higgs $(H, A)$ at large $\tan \beta$. The former appears if the masses satisfy $2 \times m_{\tilde{\chi_{1}^{0}}} \sim m_{h}$ since $h$ has a small width. The latter instead is important for moderate values of the mass parameters and benefits from the large coupling between $A$ and the third generation leading to ${\tilde{\chi_{1}^{0}}}_{1}^{\tilde{\chi_{1}^{0}}} \rightarrow b \bar{b} / \tau \bar{\tau}$.

- Stau coannihilation (Figure 4.5) dominates if the lightest stau is the next to LSP particle (NLSP). In addition to neutralino annihilation into sfermions pairs, the main coannihilation channel is now $\tilde{\chi}_{1}^{0} \tilde{\tau}_{1} \rightarrow \tau \gamma$. The scalar mass $m_{0}$ must be small and the LSP mainly a bino. The relic density is very sensitive to the mass

\footnotetext{
${ }^{1}$ This can be read as an upper limit on the mass since there might be other sources of DM
} 


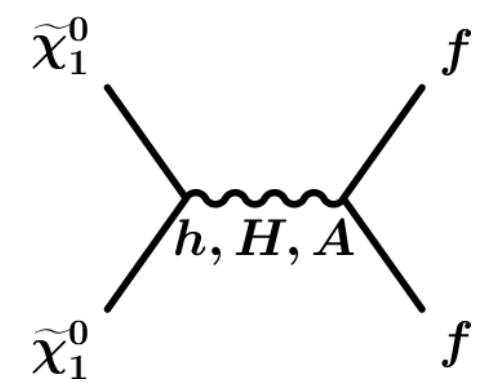

Figure 4.4. Higgs coannihilation

difference between the LSP and NLSP so the allowed region becomes a narrow strip (Figure 4.9) where $m_{\tilde{\tau}_{1}} \sim m_{{\tilde{\chi_{1}^{0}}}_{1}^{0}}$ since the coannihilation is suppressed with respect to the annihilation by a factor proportional to $e^{-\Delta m}$ where $\Delta m$ is the mass difference between the LSP and the NLSP.

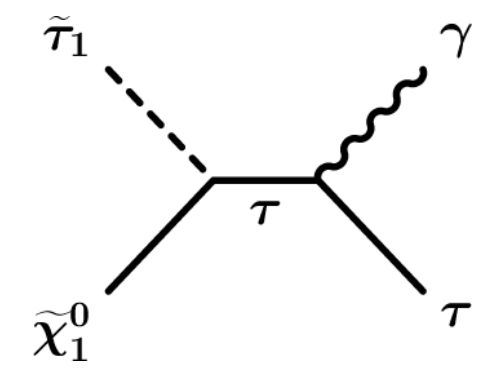

Figure 4.5. Stau coannihilation

- The focus point is the region at low $m_{\frac{1}{2}}$ and large $m_{0}(\sim 1 \mathrm{TeV})$ and the Higgsino term $\mu$ is small. The neutralino is mainly higgsino and annihilates as $\tilde{\chi}_{1}^{0} \tilde{\chi}_{1}^{0} \rightarrow W^{+} W^{-}, t \bar{t}, Z Z$. Coannihilation with charginos and heavy neutralinos are also important for very small $\mu$. Consistency with WMAP is found at large values of $\tan \beta$.

A complete review can be found in [23] [24]. 


\subsubsection{Rare decays and low energy constraints}

Besides the constraints inferred from the WMAP cosmological results, other theoretical and experimental developments can be taken into account to assess the MSSM.

- The $\Delta M_{B_{S}}$ is the oscillation frequency of the $B_{s}$ meson which has been recently measured by the CDF and D0 collaborations. While the D0 result defines a range for the oscillation [25], CDF measures $\Delta M_{B_{S}}=17.33_{-0.21}^{+0.42}($ stat $) \pm$ $0.07($ sys $) \mathrm{ps}^{-1}[26]$. Fits to the CKM parameters in the SM framework predict $\Delta M_{B_{S}}=21.5 \pm 2.6 \mathrm{pb}^{-1}$ and $\Delta M_{B_{S}}=21.7_{-4.2}^{+5.9} \mathrm{ps}^{-1}$ for UTFit [27] and CMKfitter [28] respectively. The flavor changing Higgs couplings generate contributions dependent on $\tan ^{4} \beta$. In particular, the SUSY terms have opposite sign with respect to the SM and reduce the mass difference for large values of $\tan \beta$ [29]. The current experimental results are consistent with the SM predictions within the uncertainties. These results are also consistent with the values for $M_{A}<1$ $\mathrm{TeV}$ as the SUSY contribution to the $\Delta M_{B_{S}}$ can be at most few $\mathrm{ps}^{-1}$ [30].

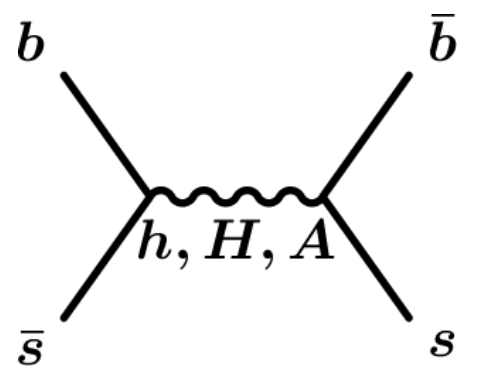

Figure 4.6. Higgs contributions to the $B_{s}$ mixing.

- The best experimental bound on the branching ratio $B_{s} \rightarrow \mu^{+} \mu^{-}$comes from the CDF collaboration as $B_{s} \rightarrow \mu^{+} \mu^{-}<1.0 \times 10^{-7}$ at $95 \%$ C.L [31]. The SM prediction is $\operatorname{BR}\left(B_{s} \rightarrow \mu \mu\right)<(3.8 \pm 1.0) \times 10^{-9}$ [32]. The $\mathrm{BR}$ is sensitive to SUSY since Higgs flavor changing neutral currents (FCNC) contributions can increase it as $\tan ^{6} \beta / \mathrm{m}_{A}{ }^{4}$. The effect is enhanced, not only at large values of $\tan \beta$, but also for moderate $\tan \beta$ and low mass Higgs [29]. 


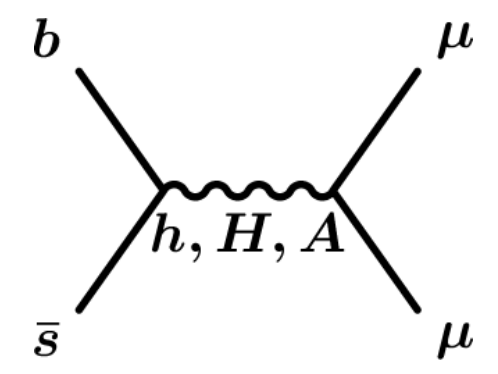

Figure 4.7. Higgs contributions to the $B_{s} \rightarrow \mu \mu$.

- Given the dependence of the $B_{s} \rightarrow \mu \mu$ upon $\tan \beta$, we can expect other processes to receive non negligible corrections if indeed the BR is in the $10^{-7}$ range. In a SUSY framework, the $\mathrm{BR}(b \rightarrow s \gamma)$ needs to be corrected for $W, H^{ \pm}$and chargino loops. In particular, the $W$ and charged Higgs interfere constructively while the chargino interference is destructive (constructive) for $\mu>0(\mu<0)$. For $\mu>0$, the chargino contribution can cancel out the $W$ and Higgs terms. Since the average result from the BELLE [33] [34], BABAR [35] [36] and CLEO [37] collaborations $(3.55 \pm 0.24 \pm 0.03) \times 10^{-4}$ agrees with the theoretical calculation $B R(b \rightarrow s \gamma)_{S M}=\left(3.61_{-0.49}^{+0.37}\right) \times 10^{-4}[38]$, very important constraints on SUSY can be set.

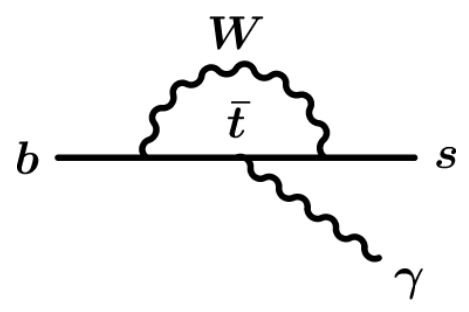

Figure 4.8. $b \rightarrow s \gamma$ via $W$ loop.

- The anomalous magnetic moment of the muon has been recently measured by the Brookhaven experiment E281 namely $a_{\mu}^{e x p}=(11659208 \pm 5.8) \times 10^{-10}$ (0.05ppm) [39]. The $e^{+} e^{-}$data show a $2.7 \sigma$ deviation from the theoretical pre- 
dictions that could be interpreted in terms of SUSY. In fact the dominant SUSY contribution enters as a chargino-sneutrino loop which depends on $\tan \beta / \mathrm{m}_{\tilde{\nu}}^{4}$. In particular its sign goes as the sign of $\mu$ implying that the $\mu>0$ region is favoured in [29]. Nevertheless, there is a disagreement on the SM calculations because of non perturbative QCD effects.

The contours of the BR $b \rightarrow s \gamma$, muon anomalous magnetic moment and cosmologically allowed regions are plotted in Figure 4.9 for $\tan \beta$ values of 10,40 and $50[40]^{2}$. The shaded green region at the bottom is excluded since the lightest $\tilde{\tau}$ becomes the LSP leading to a forbidden charged dark matter. The bulk region (low values of both $m_{\frac{1}{2}}$ and $\left.m_{0}\right)$ is favoured along with the focus point. However, as $\tan \beta$ increases, the $\mathrm{a}_{\mu}$ and rare decay BR deviate more from the SM expectations causing the bulk region to be excluded. Only the stau coannihilation channel and the focus point survive.

\subsubsection{EWK Precision measurements}

Since the EWK precision measurements might indicate New Physics as well, a $\chi^{2}$ fit of several observables was performed in [41]. The precision measurements are the $W$ mass, the Higgs lower mass limit, the effective leptonic weak mixing angle, the $\operatorname{BR}(b \rightarrow s \gamma)$ and the anomalous magnetic moment of the muon. The density of DM is taken into account by choosing an allowed $m_{0}$ value. The $\chi^{2}$ fit is calculated assuming all five observables to be independent.

$$
\chi^{2}=\sum_{n=1}^{4}\left(\frac{R_{n}^{\text {exp }}-R_{n}^{\text {theo }}}{\sigma_{n}}\right)^{2}+\chi_{M_{h}}^{2}
$$

$R_{n}^{e x p}$ denotes the experimental central value and $R_{n}^{\text {theo }}$ the mSUGRA prediction; $\chi_{M_{h}}^{2}$ is the $\chi^{2}$ for the lightest MSSM Higgs mass. The result is shown as a function of the chargino mass in Figure 4.10. This study indicates that low mass supersymmetry is preferred.

${ }^{2}$ The experimental values utilized in the study are: $0.094<\Omega h^{2}<0.129$ for the Dark Matter density; $2.2<B R(b \rightarrow s \gamma)<4.5 \times 10^{-4} ; a_{\mu}<11 \times 10^{-10}$. The LEP limits on the Higgs and Chargino mass are included in the calculation. 

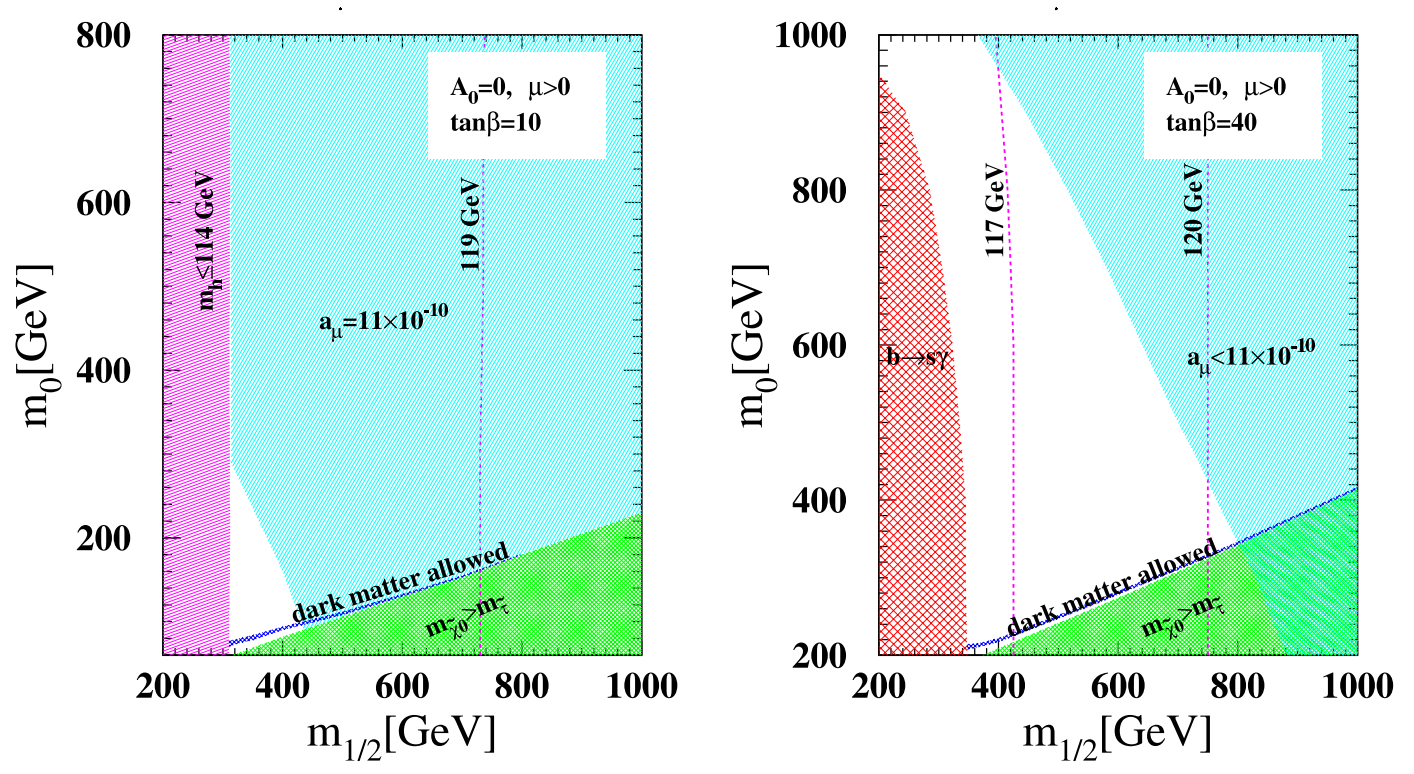

(a) $\tan \beta=10$

(b) $\tan \beta=40$

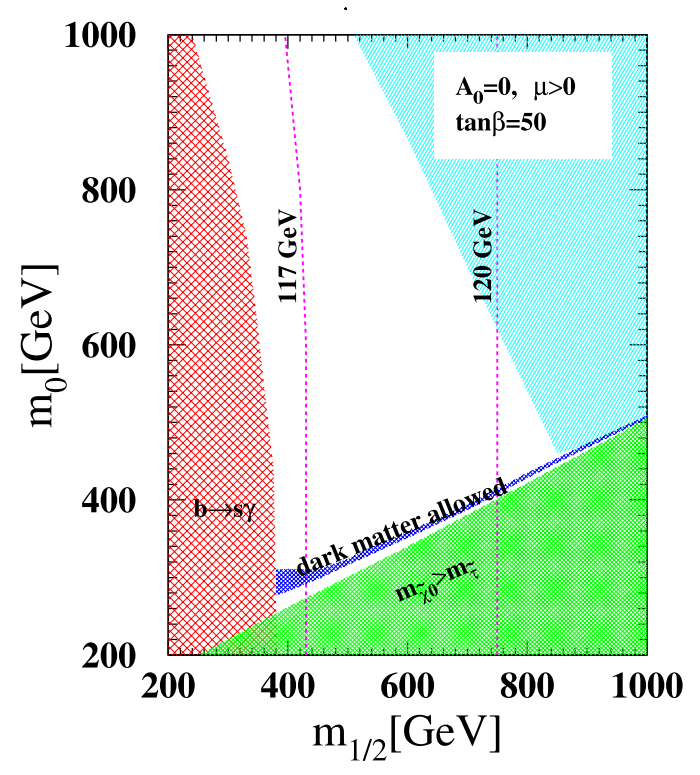

(c) $\tan \beta=50$

Figure 4.9. Preferred regions in mSUGRA parameter space. The dotted lines label different values of the Higgs mass. 


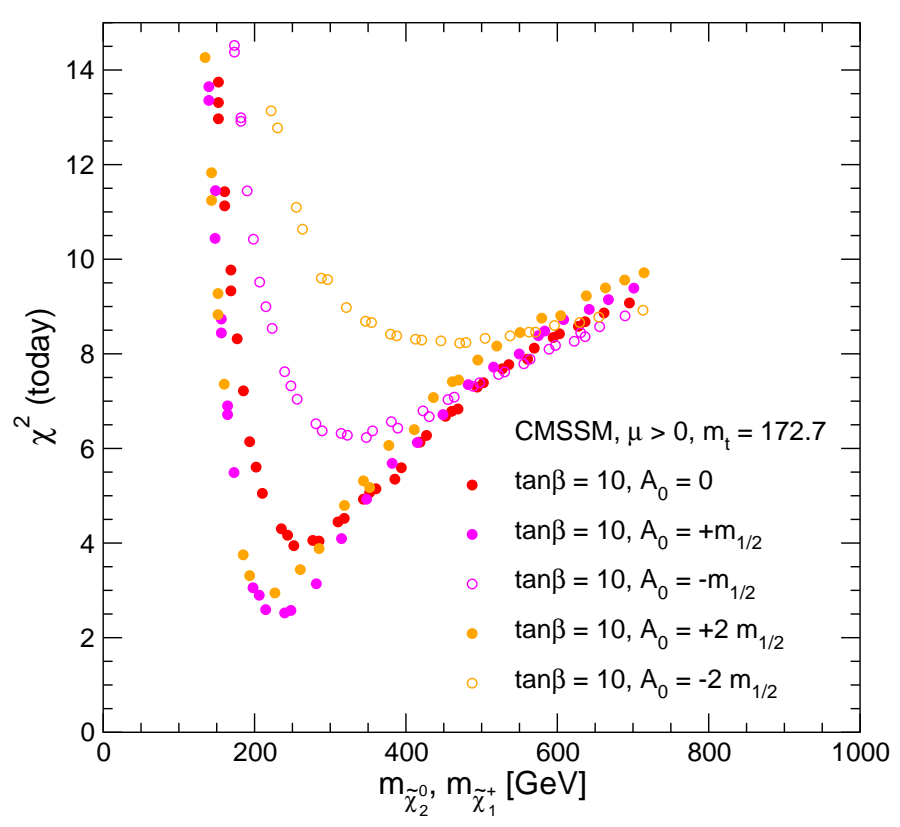

Figure 4.10. Combined likelihood $\chi^{2}$ as a function of the supersymmetric particle masses.

We should note that the indirect constraints are sensitive to the details of the model and to the uncertainties on the theoretical calculations (for instance a large uncertainty affects the dark matter calculation at large $\tan \beta$ when the RGE are run from the GUT scale down to the EWK scale). As an example, the impact of the top mass uncertainty on the mass parameters of mSUGRA is shown in Figure 4.11 [42].

\subsubsection{Direct searches for Chargino}

Unlike the indirect searches, the direct searches at colliders can provide a model independent result. We focus here on the search for chargino performed at LEP II. All LEP experiments analyzed $35.2 \mathrm{pb}^{-1}$ of data collected at $\sqrt{s}=208 \mathrm{GeV}$ looking for a SUSY signal. Since no excess over the Standard Model predictions was observed a lower mass limit on $m_{\tilde{\chi}_{1}^{ \pm}}$was set. At a $e^{+} e^{-}$collider, the limit coincides with the highest value of the masses allowed, $m_{\tilde{\chi}_{1}^{ \pm}}<\frac{\sqrt{s}}{2} \sim 104 \mathrm{GeV} / c^{2}$ [43]. It becomes weaker in some regions of the MSSM because of detection efficiency issues, 


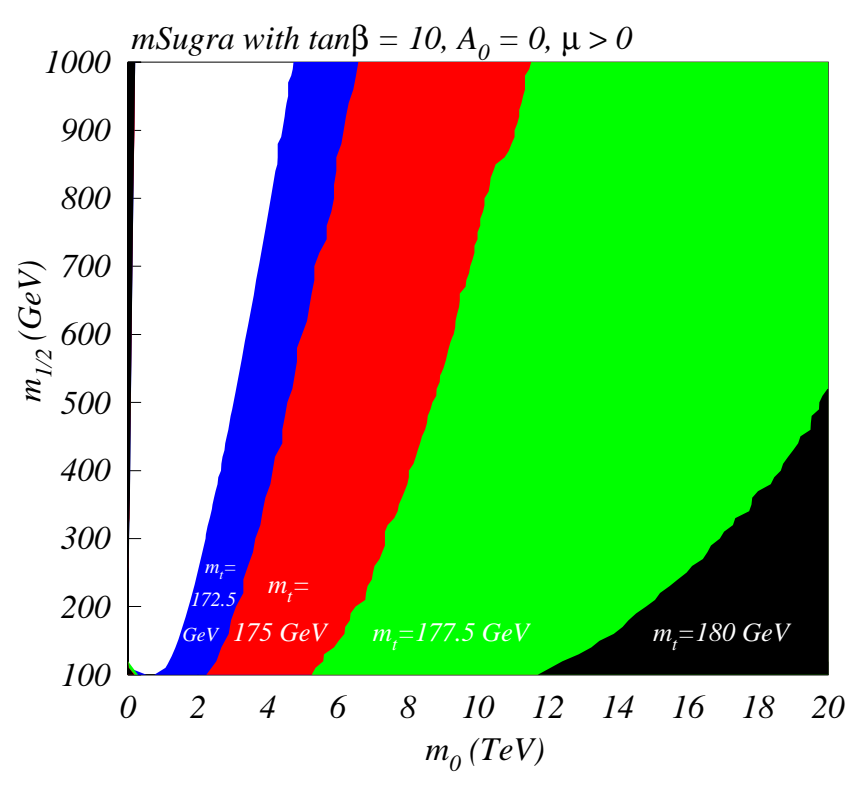

Figure 4.11. Boundary of the focus point in the mSUGRA space for different top masses.

if indirectly determined from the non observation of heavy neutralino states (through the limits on the $\mathrm{M}_{2}$ and $\mu$ parameters of the theory). For instance Figure 4.12 shows the excluded mass range, in the the MSSM with unified gauginos mass at the GUT scale. In the sneutrino low mass range, the chargino production rate is suppressed due to the destructive interference between the production channels. Similarly to the charginos, the limits on the LSP are either direct or follow indirectly from the constraints due to the non observation of charginos and neutralinos heavy states. The most stringent limit comes from the measurements of the invisible width of the Z, $m_{\tilde{\chi}_{1}^{0}}>46 \mathrm{GeV}[16]$.

\subsection{SUSY Signature at Tevatron}

We will search for SUSY looking for the associated production of chargino $\tilde{\chi}_{1}^{ \pm}$and neutralino $\tilde{\chi}_{2}^{0}$ when the two superparticles decay leptonically. The associated production is the destructive interference of two channels, the s-channel via $\mathrm{W}$ exchange 


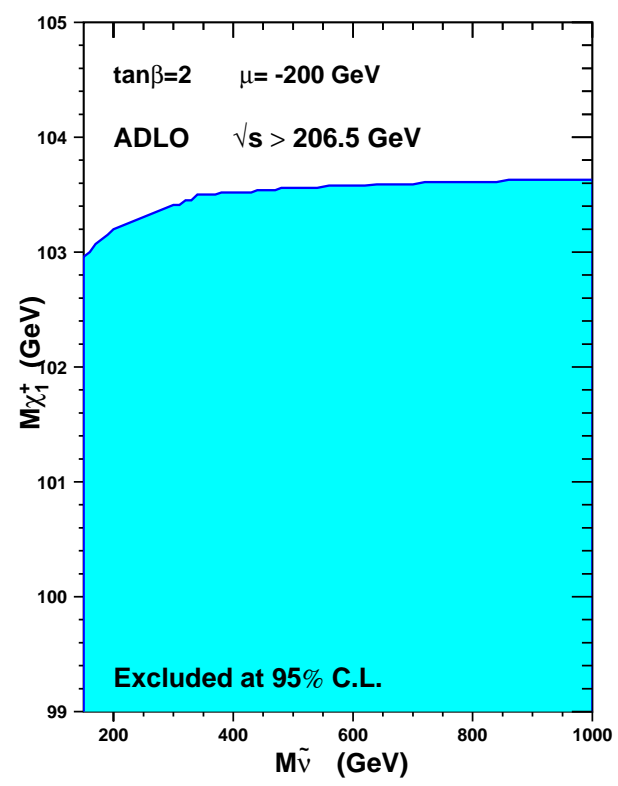

Figure 4.12. Obtained $\tilde{\chi_{1}^{ \pm}}$mass limit as a function of the sneutrino mass.

and the t-channel via squark exchange. The $s$-channel is due to the coupling between

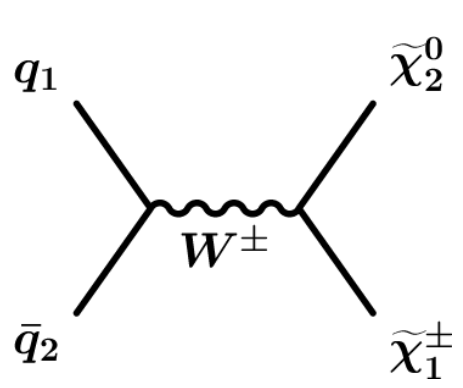

(a) s-channel

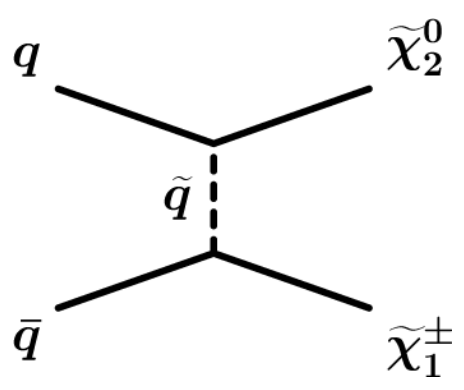

(b) t-channel

Figure 4.13. Chargino-Neutralino associated production at $\mathrm{p} \overline{\mathrm{p}}$ collider.

the wino and higgsino components of the neutralino and chargino respectively; in the t-channel the right-handed squark couples only to the bino components of neutralinos while the left-handed squark couples to both the bino and wino components of 
neutralino and chargino. Any left or right handed squarks coupling to the hgsino component is proportional to the corresponding quark mass and it can be neglected for chargino neutralino pair production. Typically the squarks are massive and the t-channel is suppressed enhancing the production cross section.

Similarly to the production cross sections, the branching ratios are also strongly dependent upon the mass of the particles in the decay. The dominant 2 body decay modes for the chargino $\tilde{\chi}_{1}^{ \pm}$are:

- $\tilde{\chi}_{1}^{ \pm} \rightarrow W^{ \pm} \tilde{\chi}_{1}^{0}$

- $\tilde{\chi}_{1}^{ \pm} \rightarrow l \tilde{\nu}$

- $\tilde{\chi}_{1}^{ \pm} \rightarrow \tilde{l} \nu$

- $\tilde{\chi}_{1}^{ \pm} \rightarrow q \tilde{q}^{\prime}$

When the two body modes are not kinematically allowed, the chargino decays to a three body final state with contributions similar to those for two body chargino decay: $(i)$ virtual gauge boson decay, (ii) virtual sfermion decay, and (iii) interference between $(i)$ and $(i i)$. The final state for a three body decay is therefore:

- $\tilde{\chi}_{1}^{ \pm} \rightarrow f \bar{f}^{\prime} \tilde{\chi}_{1}^{0}$

where $f$ and $f^{\prime}$ are fermions from the same $S U(2)$ doublet. If the sfermions are much heavier than the chargino, then $(i)$ dominates, and the decays proceed through a virtual $W$ with branching ratios similar to those of the on-shell $W$ boson. Similarly to the case of $\tilde{\chi}_{1}^{ \pm}$, the $\tilde{\chi}_{2}^{0}$ decay modes are dominated by 2-body modes if kinematically allowed:

- $\tilde{\chi}_{2}^{0} \rightarrow Z \tilde{\chi}_{1}^{0}$

- $\tilde{\chi}_{2}^{0} \rightarrow \tilde{l l}$

- $\tilde{\chi}_{2}^{0} \rightarrow \nu \tilde{\nu}$

- $\tilde{\chi}_{2}^{0} \rightarrow q \tilde{q}$ 


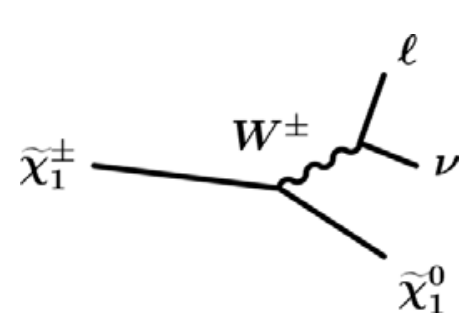

(a)

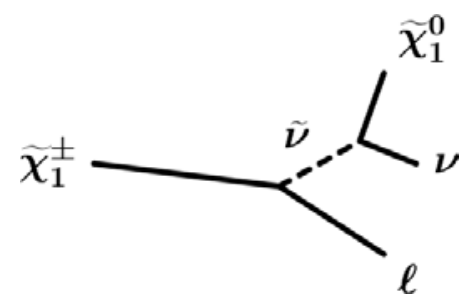

(b)

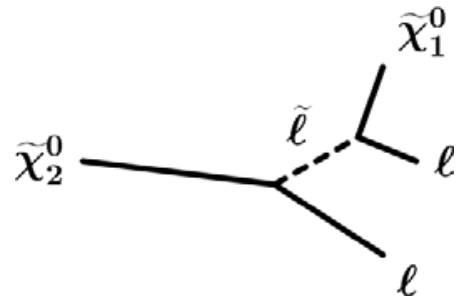

(d)

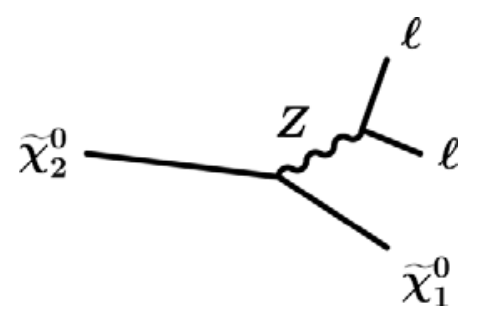

(c)

Figure 4.14. Chargino and neutralino decay modes.

- $\tilde{\chi}_{2}^{0} \rightarrow h_{0} \tilde{\chi}_{1}^{0}$

while the 3 body decays, through virtual $Z$, virtual slepton or virtual squark gives:

- $\tilde{\chi}_{2}^{0} \rightarrow f \bar{f} \tilde{\chi}_{1}^{0}$

Unlike the chargino and the $\mathrm{W}$, the decay pattern of the neutralino can differ from that of the real Z. In fact the $\mathrm{Z}$ couples only to the higgsino component of the neutralino; therefore if the neutralino have a small higgsino component, the $Z$ contribution to $\tilde{\chi}_{2}^{0} \rightarrow \tilde{\chi}_{1}^{0} f \bar{f}$ is suppressed and the one to sfermions is of comparable size.

\subsection{Sensitivity}

To assess the sensitivity for chargino-neutralino associated production at Tevatron

Run II we explore the mSUGRA parameter space in terms of $\sigma_{\tilde{\chi}_{1}^{ \pm} \tilde{\chi}_{2}^{0}} \times B R\left(\tilde{\chi_{1}^{ \pm}} \tilde{\chi_{2}^{0}} \rightarrow\right.$ 
$\ell \ell \ell$. The regions with the largest rate compatible with the constraints presented in Section 4.1 are evaluated in terms of sensitivity, defined as $\frac{S}{\sqrt{B}}$ where $\mathrm{S}$ indicates the SUSY signal and B the SM background after the detector simulation. In the current section, we provide a detailed investigation of $\sigma \times B R$ performed in the region of the parameter space called SPS1a ${ }^{3}$ [44] followed by a scan in regions preferred by the cosmological constraints.

\subsection{1 "SPS1a" Benchmark Line}

The SPS are a set of benchmark points and parameter lines in the MSSM space corresponding to different models. In particular SPS1a yields a typical mSUGRA scenario and it is parametrized as a function of $m_{\frac{1}{2}}$ with $m_{0}=0.4 \times m_{\frac{1}{2}}=-A_{0}$ and $\mu>0$. Concerning the compatibility with the constraints, the SPS1a lives mainly in the bulk of mSUGRA, however it is slightly outside the cosmological strip. But overall it is in good agreement with all the other constraints. In details, we can now look at the behavior of the masses as a function of the free parameter, the common gaugino mass $m_{\frac{1}{2}}$. As expected from the mSUGRA assumptions at the GUT scale, the chargino mass is proportional to $m_{\frac{1}{2}}$ as illustrated in Figure 4.15. while the next-tolightest neutralino is degerate to the chargino itself. The right handed sleptons are lighter than the corresponding left handed since they only interact by hyper-charge Y whereas the left handed are also affected by the $S U(2)_{L}$ interaction. Because of the small $\tan \beta$ value $(\tan \beta=5)$, the right (left) handed first and second generation leptons are almost degenerate to the $\tilde{\tau_{1}}\left(\tilde{\tau_{2}}\right)$. The cross section depends upon the inverse of the superparticles mass. As expected it decreases as the common gaugino mass parameter increases, since the chargino mass increases accordingly. It is the highest at the resonance, via real $\mathrm{W}$ and $\mathrm{Z}$, but the corresponding regions have been already excluded by LEP. In particular, $\sigma_{\tilde{\chi}_{1}^{ \pm} \tilde{\chi}_{2}^{0}}$ dominates over $\sigma \tilde{\chi}_{1}^{ \pm} \tilde{\chi}_{1}^{0}$. (Figure 4.16). Besides the dependence on the $\sigma_{\tilde{\chi}_{1}^{ \pm} \tilde{\chi}_{2}^{0}}$, the trilepton rate is determined by the branching ratio

\footnotetext{
${ }^{3}$ Slopes and Points which arose at 2001 "Snowmass Workshop on the Future of Particle Physics"
} 


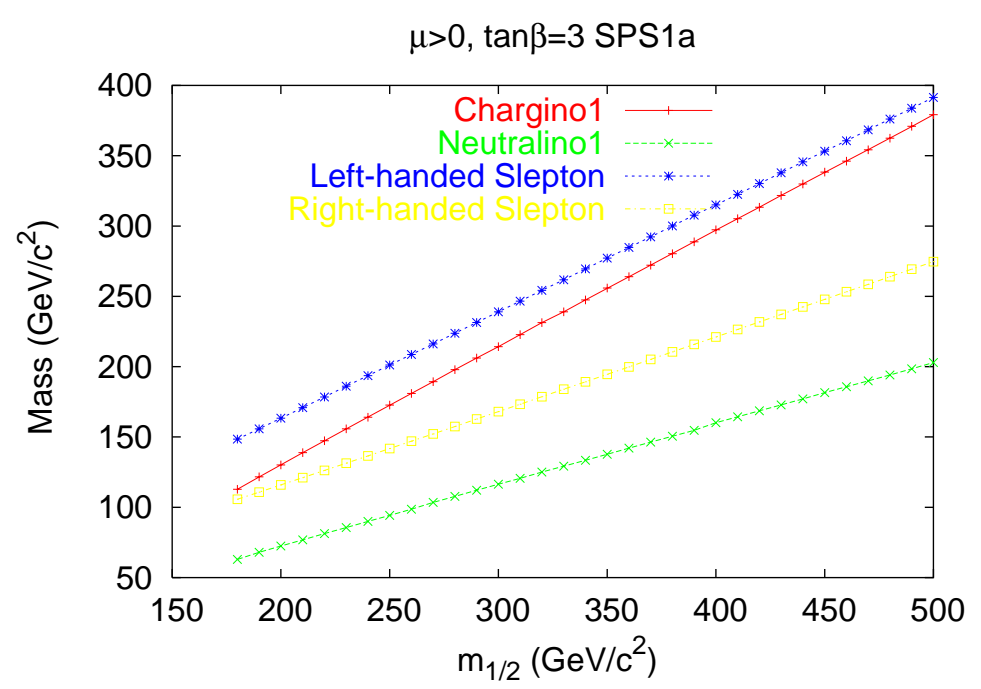

Figure 4.15. Superparticle mass as a function of the gaugino unified mass $m_{\frac{1}{2}}$.



Figure 4.16. Cross section as a function of the chargino mass.

of chargino and neutralino into leptons. Once the sparticles are produced they decay through cascades that terminate with the production of the LSP under the assumption of R-parity conservation. If $\mu$ is larger than the mass of the $S U(2)_{L}$ gaugino, $M_{2}$, the $\tilde{\chi}_{1}^{ \pm}$and $\tilde{\chi}_{2}^{0}$ are mainly gaugino like, the higgsino component being negligible. 
Therefore, the dynamical behavior is expected to be similar to the one of the $\mathrm{W}$ and $\mathrm{Z}$ bosons. In the low $m_{\frac{1}{2}}$ region, the $\tilde{\chi}_{1}^{ \pm}$branching ratio is dominated by the two body decay $\tilde{\chi}_{1}^{ \pm} \rightarrow \tilde{\tau}_{1} \nu_{\tau}$ since the $\tilde{\tau}_{1}$ is the lightest slepton, with a small contribution from the three body decay $\tilde{\chi}_{1}^{ \pm} \rightarrow f \bar{f}^{\prime} \tilde{\chi}_{1}^{0}$ via $W^{*}, \tilde{l}^{*}$ and $\tilde{H}^{*}$ as illustrated in Figure 4.17. Similarly to the $\tilde{\chi}_{1}^{ \pm}$, the branching ratio of the heavy neutralino $\tilde{\chi}_{2}^{0}$ at low $m_{\frac{1}{2}}$ is

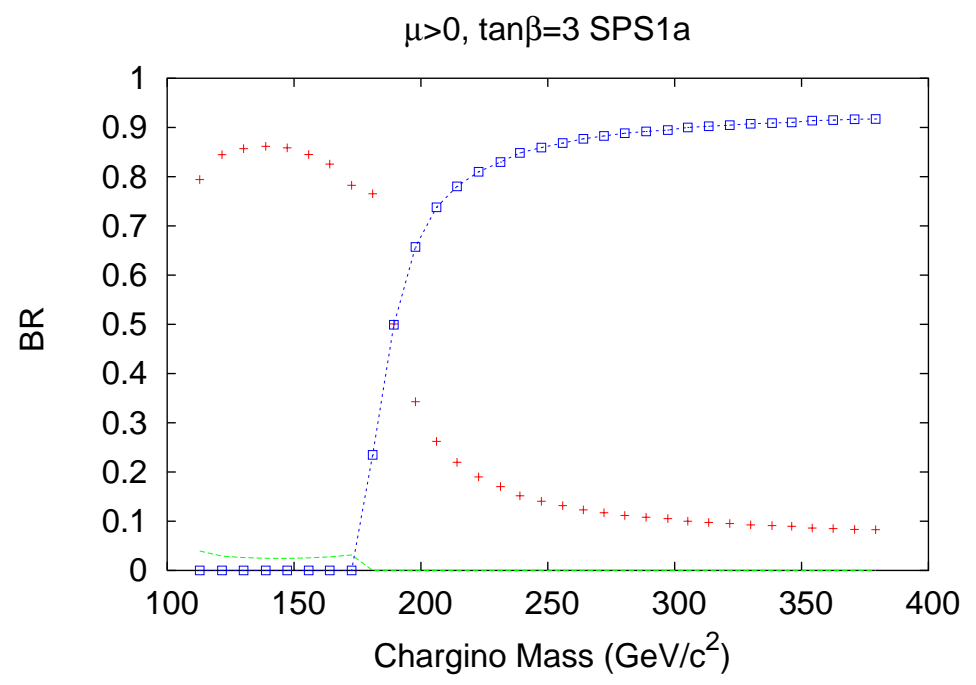

Figure 4.17. Chargino branching ratios as a function of the gaugino unified mass $m_{\frac{1}{2}}$ (Red cross symbols, chargino into $\tilde{\tau_{1}} \nu$; green solid line chargino into $f f^{\prime} \tilde{\chi}_{1}^{0}$; blue line with square symbols, chargino into $W\left(\tilde{\chi_{1}^{0}}\right)$.

dominated by the two body mode $\tilde{\chi}_{2}^{0} \rightarrow l \tilde{l}$ and by three body decay. The two modes interfere destructively, since the $\mathrm{Z}$ is off-shell. If the on-shell $\mathrm{Z}$ becomes kinematically allowed, we observe an enhancement of the branching ratio $\tilde{\chi}_{2}^{0} \rightarrow \tilde{\chi}_{1}^{0} f \bar{f}$. However the pattern is similar to the $\mathrm{Z}$ only if $\mu$ is smaller than $M_{2}$ due to the higgsino dominated $\tilde{\chi}_{2}^{0} \tilde{\chi}_{1}^{0} Z$ coupling.

The branching ratio for sleptons into leptons accompanied by the LSP is $~ 100 \%$.

Finally the effective production cross section of chargino $\tilde{\chi}_{1}^{ \pm}$and neutralino $\tilde{\chi}_{2}^{0}$ into three leptons is shown as a function of the chargino mass in Figure 4.18. It is impor- 
tant to note in this plot that the $\tau$ is not forced to decay leptonically ${ }^{4}$. The search for $\tilde{\chi}_{1}^{ \pm} \tilde{\chi}_{1}^{ \pm}$associated production is not feasible at the Tevatron because of the large $W W$ irreducible background.

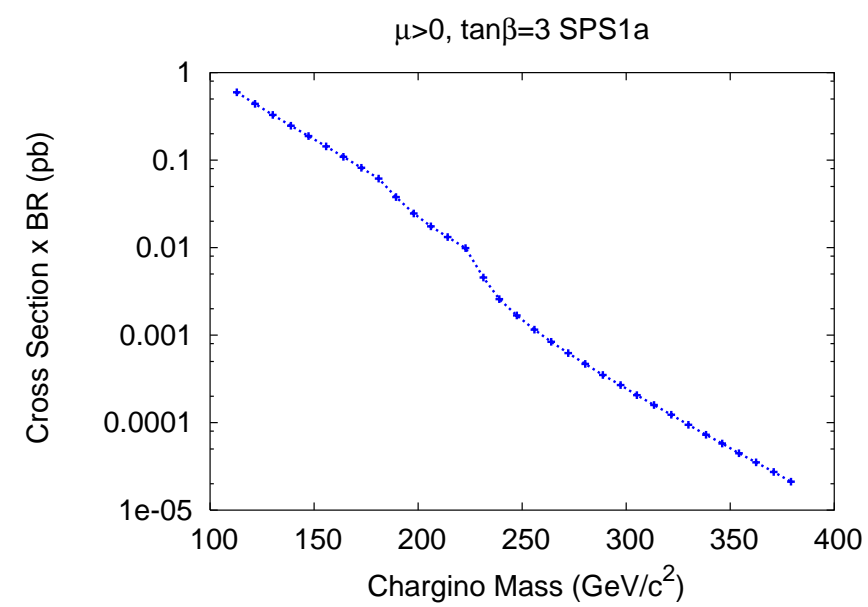

Figure 4.18. Cross section times branching ratio as a function of the Chargino mass $m_{\frac{1}{2}}$

\subsection{2 mSUGRA parameter space for $\mu>0$}

A detailed scan of the parameter space is performed by varying the parameters,

- $m_{0}$ in $[0,1000] \mathrm{GeV} / c^{2}\left(\Delta m_{0}=20 \mathrm{GeV} / c^{2}\right)$

- $m_{\frac{1}{2}}$ in $[100,1000] \mathrm{GeV} / c^{2}\left(\Delta m_{\frac{1}{2}}=20 \mathrm{GeV} / c^{2}\right)$

- $\tan \beta$ in $[2,50](\tan \beta=2,5,10,20,30,40,50)$

- $A_{0}$ in $[0,250]\left(A_{0}=0,20,50,100,150,200,250\right)$

while maintaining $\mu>0$. We obtain the best reach for $A_{0}=0$ and $\tan \beta=5$ as illustrated in Figure 4.19. Consequently, the analysis will be bench-marked with a point, benchmark point, characterized by:

\footnotetext{
${ }^{4}$ The $\tau$ decays into electrons or muons with a $\mathrm{BR} \sim 35 \%$.
} 
- $m_{\frac{1}{2}}=180 \mathrm{GeV} / c^{2} ; m_{0}=100 \mathrm{GeV} / c^{2}$

- $A_{0}=0 ; \tan \beta=5$

- $\mu>0$.

Once the analysis is carried out, the results will also be interpreted in a scenario with,

- $m_{0}=60 \mathrm{GeV} / c^{2}$

- $A_{0}=0 ; \tan \beta=3$

- $\mu>0$.

This set of parameters is referred to as our default benchmark line since we let the gaugino mass vary from 162 to $230 \mathrm{GeV} / c^{2}$.

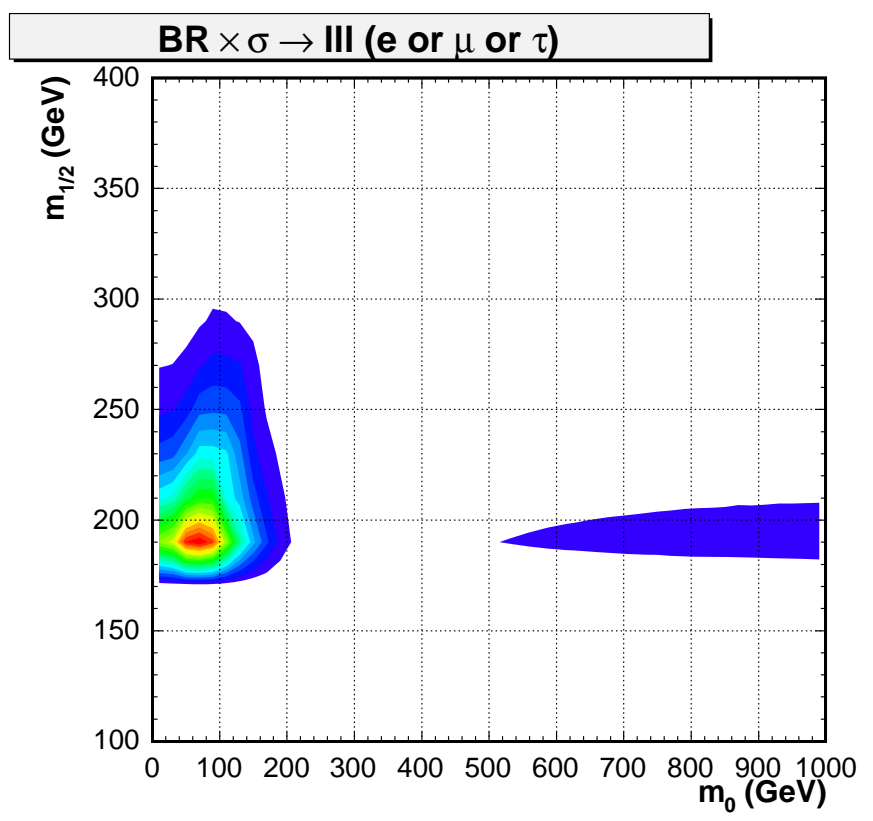

Figure 4.19. Cross section times branching ratio as a function of the gaugino and scalar unified mass $\left(\tan \beta=5 ; A_{0}=0 ; \mu>0\right)$. The LEP excluded regions are not investigated. The $\tau$ is not forced to decay leptonically. 


\subsubsection{Bulk of the mSUGRA parameter space}

We scan over several points in the parameter space at small $m_{\frac{1}{2}}$ to insure a large cross section $\sigma_{\tilde{\chi}_{1}^{ \pm} \tilde{\chi}_{2}^{0}}$ and small $m_{0}$ to gain a large branching ratio into sleptons. In order to enhance the sensitivity to the first and second generation leptons we maintain $\tan \beta=3$. Since the mSUGRA parameters are tightly constrained when the higgsino term is negative, we fix $\mu>0$. For this set of parameters we then vary the trilinear coupling $A_{0}$ and determine the behavior of the effective cross section (Figure 4.20). The soft SUSY terms introduce couplings between the Higgs and the fermions which are very important for the third generation. These couplings affect the mass of the superparticles and indirectly the production cross section. In Figure 4.21 the results are presented for different values of $m_{0}=60,70,80 \mathrm{GeV} \mathrm{c} c^{2}$ (blue dashed line) and compared to our benchmark line (red solid line) with $m_{0}=62 \mathrm{GeV} / c^{2}$.

\subsubsection{Cosmologically preferred regions}

Similarly to the investigation described earlier, we also measure the effective cross sections in the following regions.

- Stau coannihilation: $m_{0}=5.9+0.3 \times m_{\frac{1}{2}}+3.0 \times 10^{-5} \times m_{\frac{1}{2}}^{2}$ for $\tan \beta=10, A_{0}=0$ $\mathrm{GeV}$ and $\mu>0$.

- Higgs funnel: $m_{0}=815-2.2 \times m_{\frac{1}{2}}+3.3 \times 10^{-3} \times m_{\frac{1}{2}}^{2}-1.5 \times 10^{-6 \times} m_{\frac{1}{2}}^{3}$ for $\tan \beta=50, A_{0}=0 \mathrm{GeV}$ and $\mu>0$.

- Focus point: $m_{0}=3020+2.7 \times m_{\frac{1}{2}}-1.0 \times 10^{-4} \times m_{\frac{1}{2}}^{2}$ for $\tan \beta=50, A_{0}=0$ and $\mu>0$.

- Bulk region indicated by the $\chi^{2}$ fit of the EWK precision measurements: $m_{0}=60$ and $100 \mathrm{GeV} / c^{2}, \tan \beta=3, A_{0}=2 \times m_{\frac{1}{2}}$. 

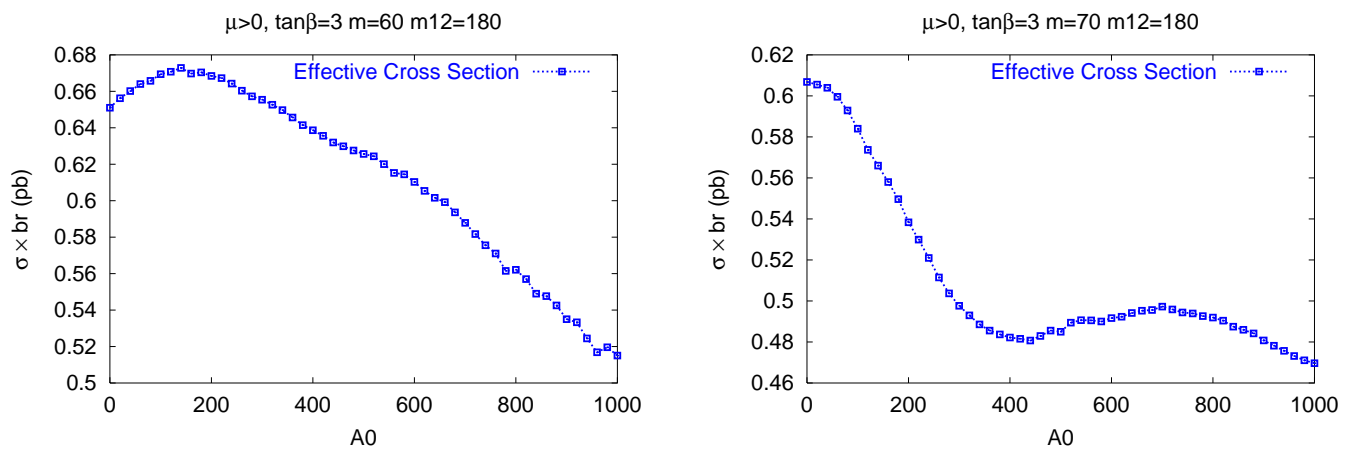
(a) $m_{0}=60 \mathrm{GeV} / c^{2} ; m_{\frac{1}{2}}=180 \mathrm{GeV} / c^{2}$
(b) $m_{0}=70 \mathrm{GeV} / c^{2} ; m_{\frac{1}{2}}=180 \mathrm{GeV} / c^{2}$

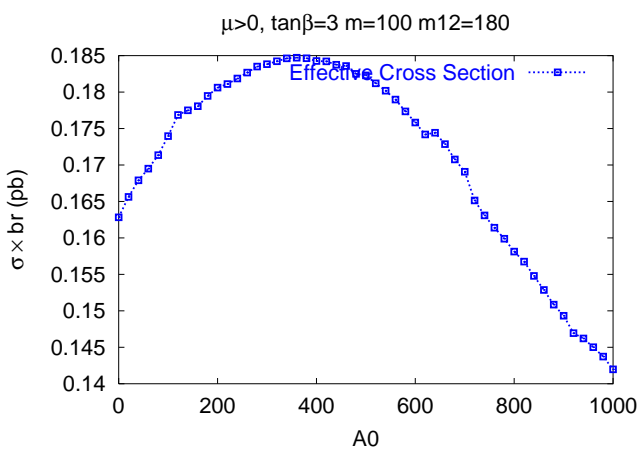

(c) $m_{0}=100 \mathrm{GeV} / c^{2} ; m_{\frac{1}{2}}=180 \mathrm{GeV} / c^{2}$

Figure 4.20. $\sigma \times B R$ as a function of $A_{0}$.

Figure 4.22(a) 4.22(d) shows the effective cross section as a function of the chargino mass, compared to the benchmark line. The results confirm that the benchmark line is the most promising scenario. 


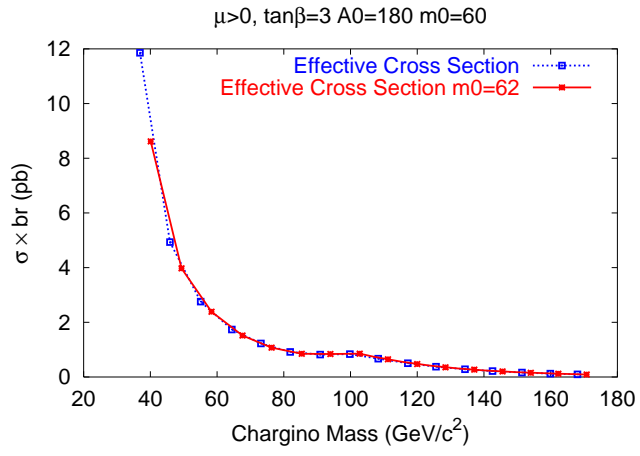

(a) $m_{0}=60 \mathrm{GeV} / c^{2} ; A_{0}=180 \mathrm{GeV}$

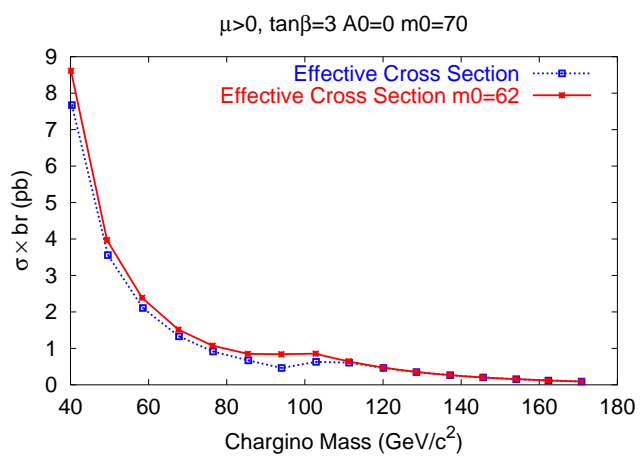

(c) $m_{0}=70 \mathrm{GeV} / c^{2} ; A_{0}=0 \mathrm{GeV}$

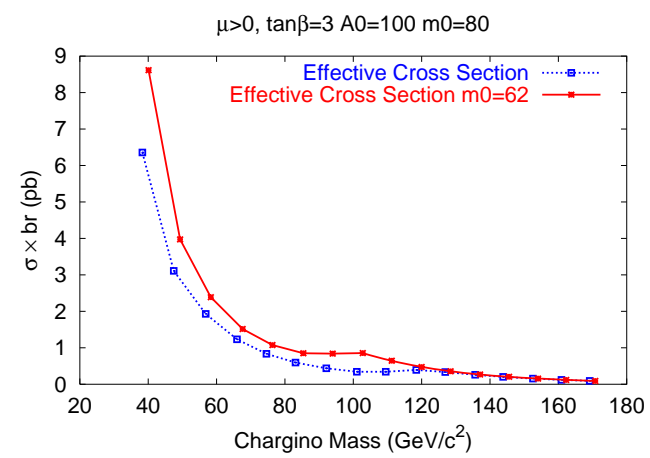

(e) $m_{0}=80 \mathrm{GeV} / c^{2} ; A_{0}=100 \mathrm{GeV}$

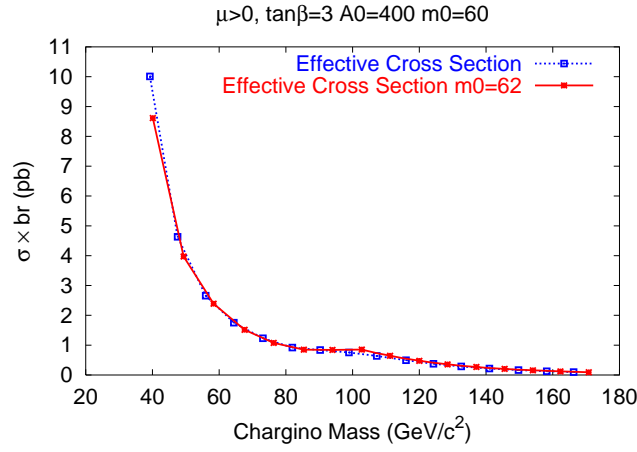

(b) $m_{0}=60 \mathrm{GeV} / c^{2} ; A_{0}=400 \mathrm{GeV}$

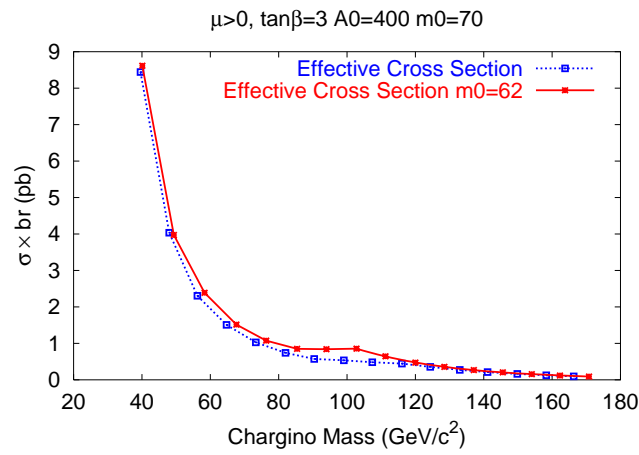

(d) $m_{0}=70 \mathrm{GeV} / c^{2} ; A_{0}=400 \mathrm{GeV}$

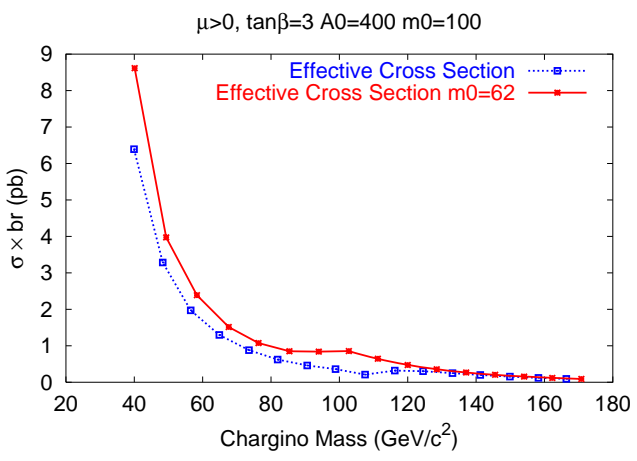

(f) $m_{0}=80 \mathrm{GeV} / c^{2} ; A_{0}=400 \mathrm{GeV}$

Figure 4.21. $\sigma \times B R$ as a function of the chargino mass. 


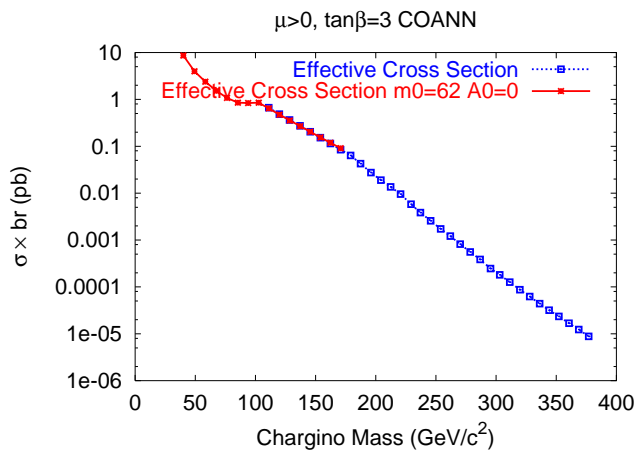

(a) Coannihilation Region

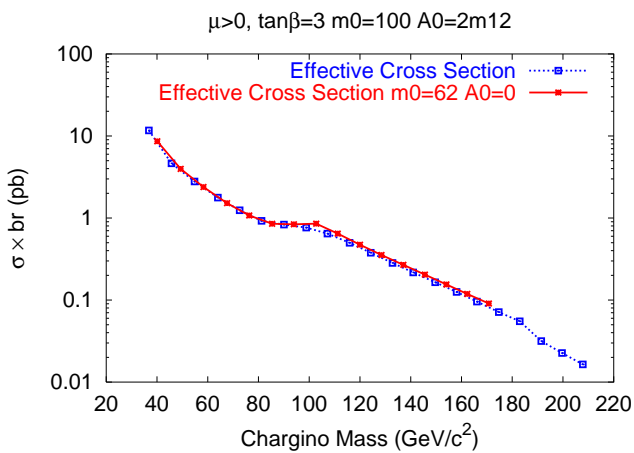

(c) EWK preferred region; $m_{0}=60$ $\mathrm{GeV} / c^{2}$

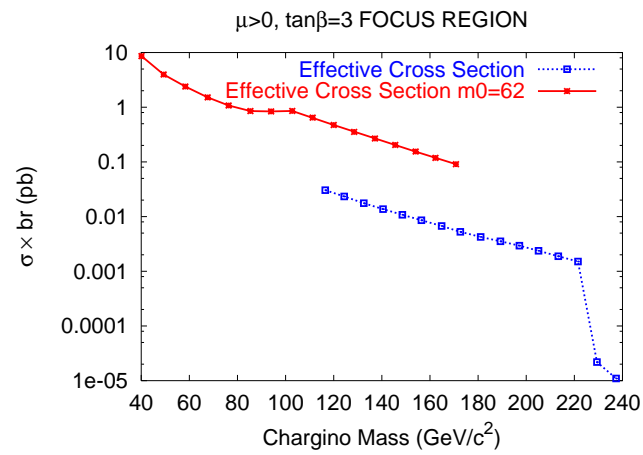

(b) Focus Point

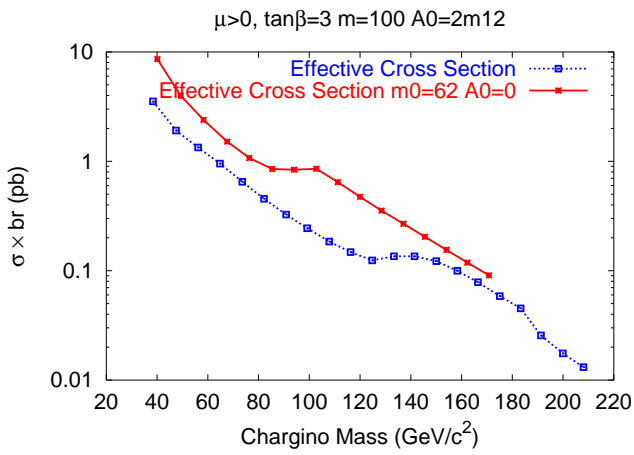

(d) EWK preferred region; $m_{0}=100$ $\mathrm{GeV} / c^{2}$

Figure 4.22. $\sigma\left(q \bar{q} \rightarrow \tilde{\chi}_{1}^{ \pm} \tilde{\chi}_{2}^{0}\right) \times B R\left(\tilde{\chi}_{1}^{ \pm} \tilde{\chi}_{2}^{0} \rightarrow \ell \ell \ell \tilde{\chi}_{1}^{0}\right)$ as a function of the chargino mass. 


\section{The Tevatron and the CDF Experiment}

Located about 35 miles west of Chicago, Illinois, the Fermi National Accelerator Laboratory (Fermilab) is one of the main particle physics facilities in the world. It was built in the late 1960's by the Department of Energy. During the past 40 years several experiments at Fermilab have made important contributions to the understanding of the Standard Model. In 1977, the E288 experiment observed a new particle, the Upsilon, composed of a new kind of quark and its antimatter partner, giving the first evidence for the existence of the bottom quark. In 1995 the CDF and D0 experiments at the Tevatron proton-antiproton Collider completed the quark sector of the SM with the observation of the top quark. In July 2000, DONUT announced the first direct observation of the tau neutrino, filling the final slot in the lepton sector of the Standard Model. The Run II of the Tevatron Collider was inaugurated in March 2001 and it was designed to enhance the goals of High Energy Physics frontiers, with a complete upgrade of the Fermilab accelerator complex. In this Chapter we briefly describe the Tevatron accelerator and the Collider Detector at Fermilab (CDF).

\subsection{The Tevatron}

The Fermilab accelerator complex shown in Figure 5.1 is composed of five different accelerators: the Cockroft-Walton, the Linac, the Booster, the Main Injector, and the Tevatron. They work in cascade and accelerate bunches of protons and antiprotons in opposite directions to energies of $980 \mathrm{GeV}$, resulting in a total energy per $\mathrm{p} \overline{\mathrm{p}}$ collision of $1.96 \mathrm{TeV}$. The center of mass-energy determines the value of the cross section for the physical processes and an upper limit for the mass of particles that can be produced at this machine. Another key parameter in collider experiments is 


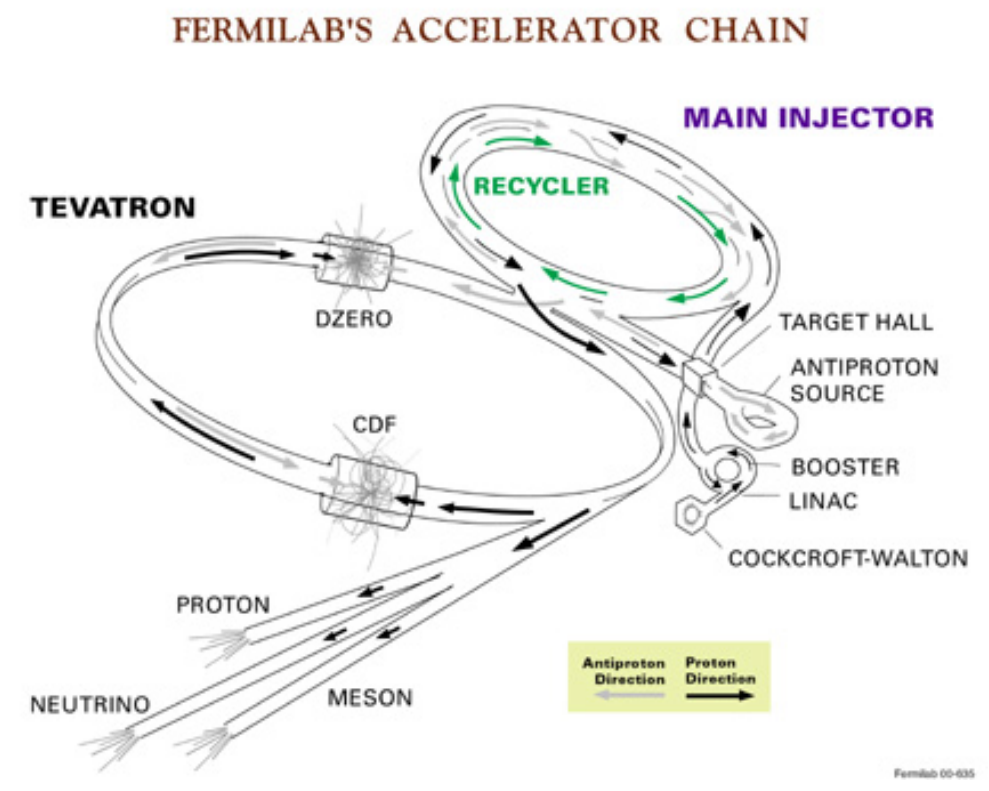

Figure 5.1. A schematic drawing of the Fermilab accelerator complex.

the luminosity $L$, defined as the the number of collisions per unit time and unit area ${ }^{1}$. From the luminosity we can calculate the number of events as:

$$
N=\sigma \cdot \int_{\Delta T} L d t=\sigma \cdot \mathcal{L}
$$

where $\sigma$ is the production cross section of the process of interest and $\mathcal{L}$ the "integrated luminosity" collected in the time interval $\Delta T$. The Run II upgrades of the accelerator were driven by the equation above. First, the center of mass-energy was increased from the Run I value of 1.8 to $1.96 \mathrm{GeV}$ which translated into larger production cross sections. But the most important upgrade is the higher instantaneous luminosity $\mathcal{L}_{\text {inst }}$, where $\mathcal{L}_{\text {inst }}$ is determined as

$$
\mathcal{L}_{\text {inst }} \sim \frac{f B N_{p} N_{\bar{p}}}{2 \pi\left(\sigma_{p}^{2}+\sigma_{\bar{p}}^{2}\right)}
$$

where $f$ is the revolution frequency, $B$ the number of bunches in each beam, $N_{p}$ $\left(N_{\overline{\mathrm{p}}}\right)$ the number of protons (antiprotons) per bunch and $\sigma_{p}\left(\sigma_{\overline{\mathrm{p}}}\right)$ the transverse beam

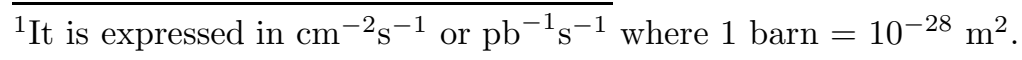


sizes at the interaction point. The higher $\mathcal{L}_{\text {inst }}$ is achieved by increasing the number of bunches: the Tevatron is presently operating at $36 \times 36$ bunches with a bunch crossing of 396 ns. Figure 5.2 shows that the number of overlapping collisions for a given instantaneous luminosity, decreases as the number of bunches increases.

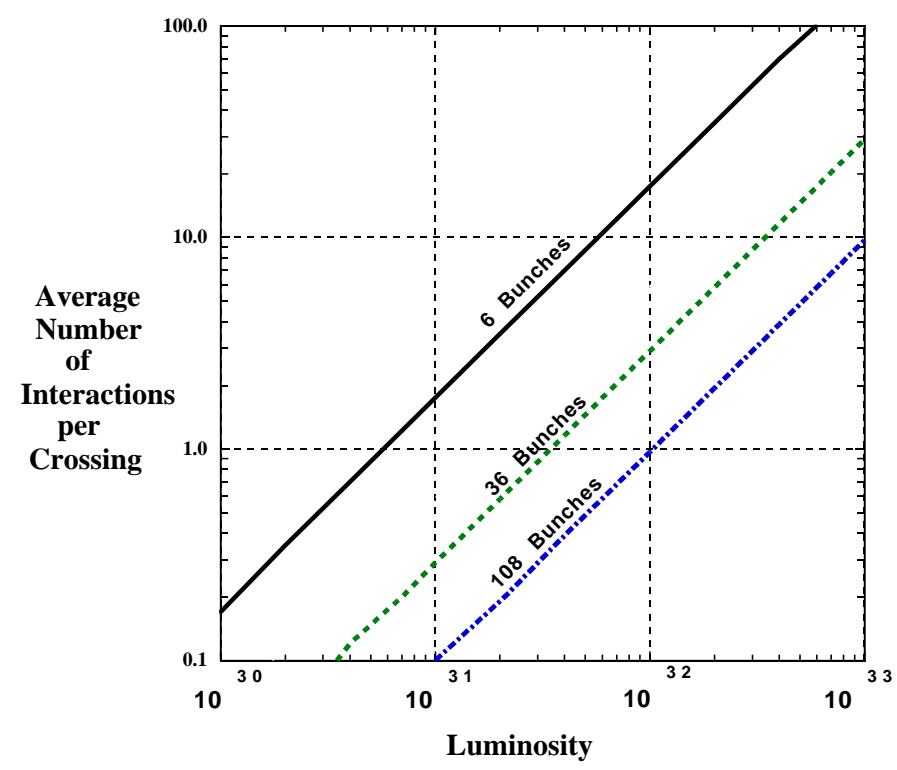

Figure 5.2. Average number of interactions per bunch crossing as a function of the instantaneous luminosity for different beam conditions.

\subsubsection{Production and boosting of protons}

Protons are extracted from negative ionized hydrogen gas. Hydrogen molecules are passed through a magnetron, which extracts ions, consisting of two electrons and one proton, and accelerates them to the energy of $25 \mathrm{keV}$. These ions are then accelerated using the potential difference supplied by the Cockroft-Walton preaccelerator, which produces a beam of $750 \mathrm{keV} \mathrm{H}^{-}$ions. The $\mathrm{H}^{-}$ions subsequently enter the second stage of the accelerator process, the Linac. The Linac is a linear accelerator about 150 m long, which increases the energy of the $\mathrm{H}^{-}$to $400 \mathrm{MeV}$, by means of radio frequency cavities. The beam of ions is then injected in the Booster, a small synchrotron having 
$150 \mathrm{~m}$ of diameter, where ions are merged with the already circulating protons. The combined beam is focused on a thin carbon sheet, which strips the $\mathrm{H}^{-}$of their two electrons. Using radio frequency cavities, the protons are packed into bunches and accelerated to $8 \mathrm{GeV}$. Protons are now ready to be transfered into the Main Injector.

\subsubsection{Main Injector}

The Main Injector is a synchrotron of $3 \mathrm{~km}$ in circumference, where protons and antiprotons are accelerated to $150 \mathrm{GeV}$ before they enter the adjacent Tevatron. The main feature of this synchrotron is the large particle acceptance to accommodate bunches from the Booster.

\subsubsection{Production and cooling of antiprotons}

Antiprotons are produced by dumping a $120 \mathrm{GeV}$ proton bunch extracted from the Main Injector into a nickel target. Among the variety of particles generated in the collision, antiprotons of about $8 \mathrm{GeV}$ energy are produced and collected through lithium lenses and dedicated magnetic field. Subsequently antiprotons are sent to the Accumulator, where they are further cooled and stacked into bunches.

Both a stochastic and an electron cooling systems are used to cool antiproton bunches prior to transfers to the Tevatron collider. The implementation of electron cooling significantly increased the number of antiprotons stored in the Recycler; as a consequence, the antiproton production rate in the Accumulator improved, increasing the luminosity of the Tevatron collider ${ }^{2}$. When a sufficient number of antiprotons is

\footnotetext{
${ }^{2}$ The goal of the electron cooling in the Recycler Ring is to cool $9 \mathrm{GeV}$ antiprotons by mixing them in a 20 -m long cooling section with a cold beam of $4.8 \mathrm{GeV}$ electrons. The electron cooling is based on overlapping a stored beam with a monochromatic and parallel electron beam in one of the straight sections of a storage ring. The velocity of the electrons is made equal to the average velocity of the antiprotons. The antiprotons undergo Coulomb scattering in the electron gas and loose energy, which is transferred from the antiprotons to the co-streaming electrons until some thermal equilibrium is attained. Since the number of particles stays the same and the space coordinate are reduced the temperature decreases.
} 
available, they are accelerated to $150 \mathrm{GeV}$ and, together with the protons, transferred to the Tevatron for the ultimate acceleration stage before the collisions.

\subsubsection{Tevatron}

The Tevatron is a $6 \mathrm{~km}$ circumference proton-antiproton synchrotron collider located a few meters underground. It makes use of super-conducting dipole magnets and quadrupole focusing magnets to provide a stable circular orbit for the protons and antiprotons circulating in the same beam pipe in opposite directions. Once 36 bunches of protons and 36 of antiprotons ("a store") are circulating in the Tevatron, the beam energies are ramped up to $980 \mathrm{GeV}$. The two beams, kept spatially separated during the acceleration stage, are now made to collide in two regions around the ring. In these regions, where the two experiments D0 and CDF are located, a collision takes place every $396 \mathrm{~ns}$. In particular at CDF, the luminous region has a dispersion of about $30 \mathrm{~cm}$ in the directions of the beams $\left(\sigma_{z} \sim 30 \mathrm{~cm}\right)$ due to the geometrical configuration of the bunches. The transverse section of the beams is approximately circular and has a gaussian dispersion $\sigma_{t}^{\text {beam }} \sim 30 \mu \mathrm{m}$.

\section{Tevatron performance and future prospective}

The accelerator has been running since March 2001. Many problems were identified during the start-up period. The efficiency of the machine was severely limited, by beam-beam effects and major antiproton losses occurred during the squeeze. Most of these losses have been overcome by changing the helices to increase the beam separations, with smaller antiproton emittance. In addition, other improvements have been implemented upgrading the accumulator stochastic cooling, as mentioned earlier, and modifying the proton beam loading compensation in the Main Injector. At present the initial instantaneous luminosity is achieving record values of $1.7 \times 10^{32} \mathrm{~cm}^{-2} \mathrm{~s}^{-1}$ as shown in Figure 5.3(a), which is very close to what was nominally expected in Run 
$\mathrm{II}^{3}$. A $1 \mathrm{fb}^{-1}$ sample has been collected by Summer 2005. However, due to the problems of the first 2 years of running, the delivered integrated Luminosity is lower than the original expectation, although it will still be possible to reach between 4.4-8.6 $\mathrm{fb}^{-1}$ by 2009 if the performance of the machine keep improving.

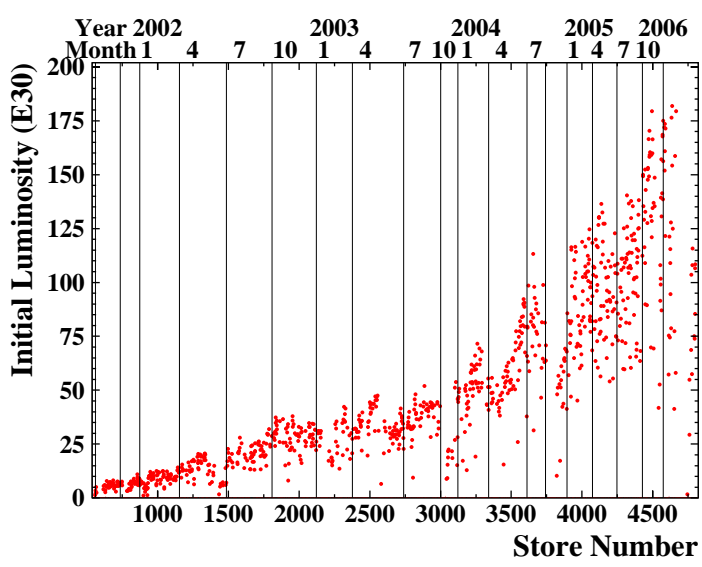

(a) Instantaneous luminosities at the start of the stores.

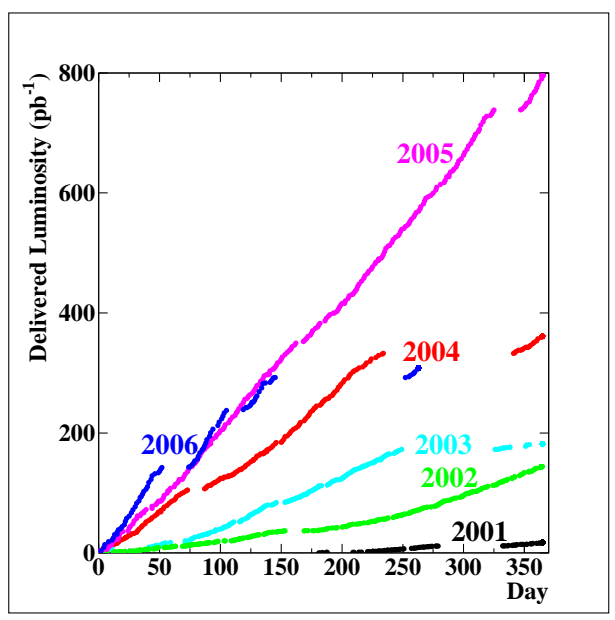

(b) Delivered Luminosity by the Tevatron.

Figure 5.3. Tevatron Performance through Spring 2006.

\subsection{Collider Detector at Fermilab}

The CDF II detector [45], located at one of the six nominal interaction regions of the Tevatron, is a general purpose detector which combines precision charged particle tracking with projective calorimetry and fine grained muon detection. A schematic view of the CDF II detector is given in Figure 5.4. It measures approximately $27 \mathrm{~m}$ from end-to-end, is about $10 \mathrm{~m}$ high, and weighs over 5000 tons. The right-handed coordinate system in Figure 5.5 is defined with respect to the proton beam direction which gives the $\mathrm{z}$-axis. The $\mathrm{y}$-axis points vertically upward, and the $\mathrm{x}$-axis lies in the ${ }^{3}$ The nominal luminosity is $0.9 \times 10^{32} \mathrm{~cm}^{-2} \mathrm{~s}^{-1}$ for first 3 years and $1.6 \times 10^{32} \mathrm{~cm}^{-2} \mathrm{~s}^{-1}$ afterwards. 


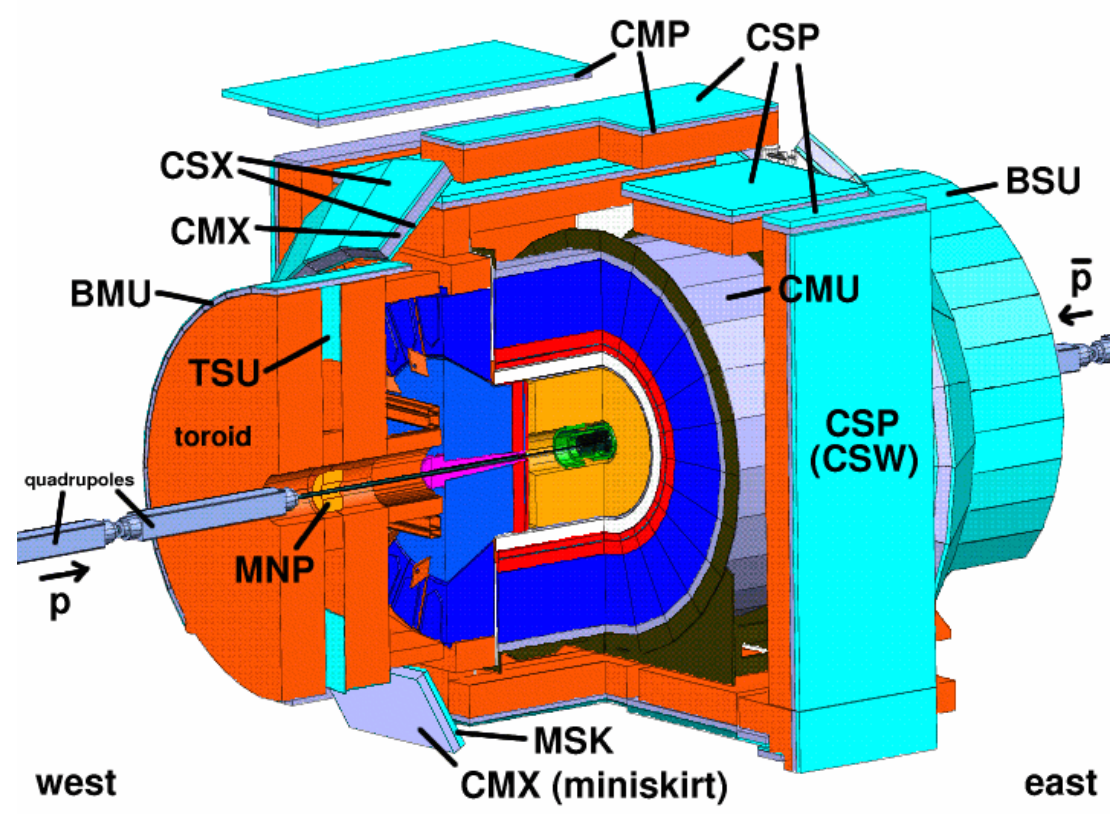

Figure 5.4. CDF Run II detector view.

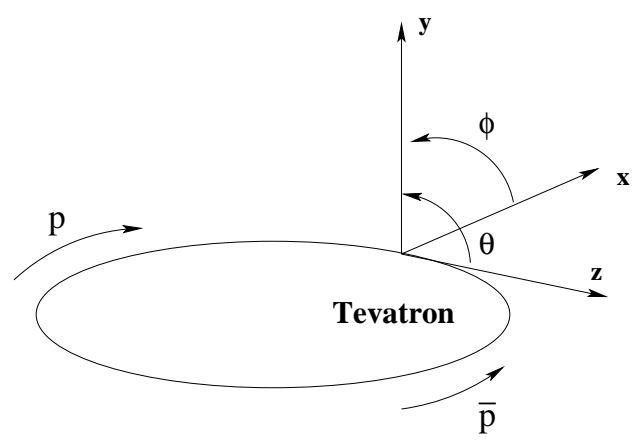

Figure 5.5. Coordinate system.

plane defined by the Tevatron ring. The origin of the coordinate system is placed at the nominal interaction point that coincides with the center of the detector. The azimuthal angle $(\phi)$ is measured counterclockwise from the Tevatron plane, the polar angle $(\theta)$ is defined with respect to the positive z-axis, and the pseudo-rapidity $(\eta)$ is defined as $\eta=-\ln \left[\tan \left(\frac{\theta}{2}\right)\right]$. To work with the new high luminosity and therefore with the high collision rate of Run II the CDF experiment underwent significant 
upgrades with many new features. In the following sections a description of the various sub-detectors involved in this analysis is given as well as an overview of the major changes in the trigger and in the data acquisition system.

\subsubsection{Central Tracking System}

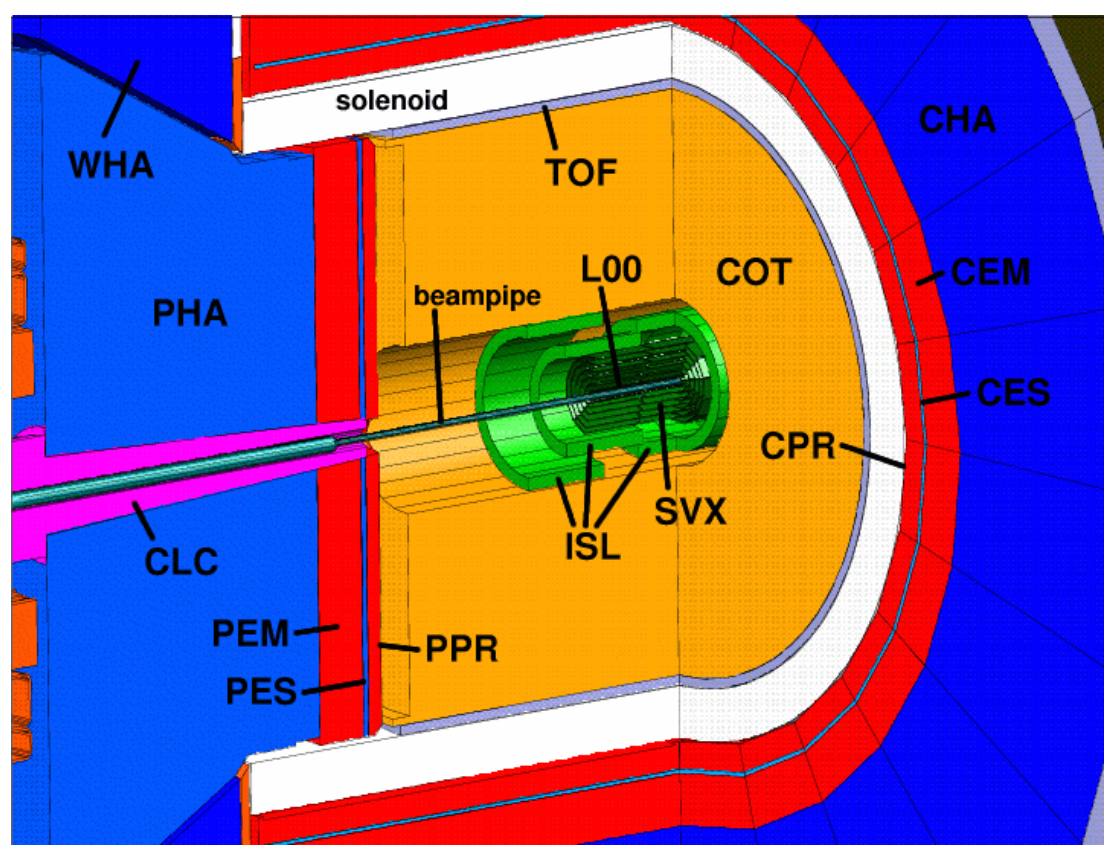

Figure 5.6. CDF Run II tracking system view.

The Central Tracking System shown in Figure 5.6 is designed to reconstruct the trajectories of charged particles coming from the interaction vertex. The whole system is composed of four subsystems: the Layer 00 (L00, located at the beam pipe), the Silicon Vertex Detector (SVXII), the Intermediate Silicon Layers (ISL) and the Central Outer Tracker (COT). The two innermost Silicon detectors provide an excellent 3D vertex measurement within $|\eta|<2$, ISL allows forward tracking in a wider $\eta$ range than the $\mathrm{COT}$, and the COT completes the tracking in the central region $(|\eta|<1)$ before the particles reach the calorimeter. All these devices are placed inside a solenoidal magnetic field of 1.41 Tesla parallel to the beam line. The field 
is uniform to $0.1 \%$ in the region $|z|<150 \mathrm{~cm}$ and $r<150 \mathrm{~cm}$. The solenoid and the cryogenic equipment represent 0.85 radiation length $\left(X_{0}\right)$. The curvature of the charged particles in the magnetic field allows the extraction of precise information on the particle momentum as well as the sign of their charge. Starting from the Lorentz force, the transverse momentum of a reconstructed track is given by $p_{T}=B \cdot|q| \cdot e \cdot \rho$, where $q$ is the particle charge, $e$ is electron charge, $B$ the magnetic field and $\rho$ the radius of the helicoidal trajectory.

The impact parameter resolution of SVXII+ISL is $40 \mu \mathrm{m}$, including $30 \mu \mathrm{m}$ beam size, and the $Z_{0}$ resolution is $70 \mu \mathrm{m}$. The COT hit position resolution is $140 \mu \mathrm{m}$ and the momentum resolution is $\sigma\left(p_{T}\right) / p_{T}{ }^{2}=0.0015(\mathrm{GeV} / c)^{-1}$.

\section{Silicon Vertex Detector (SVXII)}

SVXII consists of three $32 \mathrm{~cm}$ long cylindrical barrels; the total length is $96 \mathrm{~cm}$, with $70 \%$ geometrical acceptance. The pseudo-rapidity coverage is $|\eta|<2$. Each barrel is formed by five layers (L0 to L4) of double sided micros-trip silicon detectors. The innermost layer is placed at a radius of $2.4 \mathrm{~cm}$, while the outermost is at a radius of $\sim 10.7 \mathrm{~cm}$. Three of the five layers have micro-strips aligned to the beam on one side of the silicon wafer to provide $r-\phi$ information, and orthogonal to it on the other side to provide $r-z$ information. On two layers the micro-strips are instead tilted at small angle and the sensors denoted small angle stereo or SAS. The $r-\phi$ information is also denoted as axial. The micro-strips are spaced in $r-\phi$ by approximately 60 to 65 microns, depending on the layer, with implant widths of 14 to 15 microns. The stereo micro-strips are spaced by 141, 125.5, 60, 141, 65 microns for L0 to L4, and have implant widths of 20 microns for the $r-z$ and 15 microns for the SAS layers. The sensors are mounted on $29 \mathrm{~cm}$ long mechanical units called ladders; the ladders, each one divided into two electrical units, have staggered radii and provide overlap and redundancy. The readout electronics consists of hybrid chips mounted directly on the silicon surface at each end of the half-ladder. The choice of electronics 
located on the silicon sensors was made to reduce the dead-time area between the barrels. The electronics inside the detector adds material within the active sensitive volume because of cables and cooling tubes. This increases the multiple scattering of a particle worsening the pattern recognition capability. To partially mitigate this effect another layer of silicon at small radius L00 was added to SVX II.
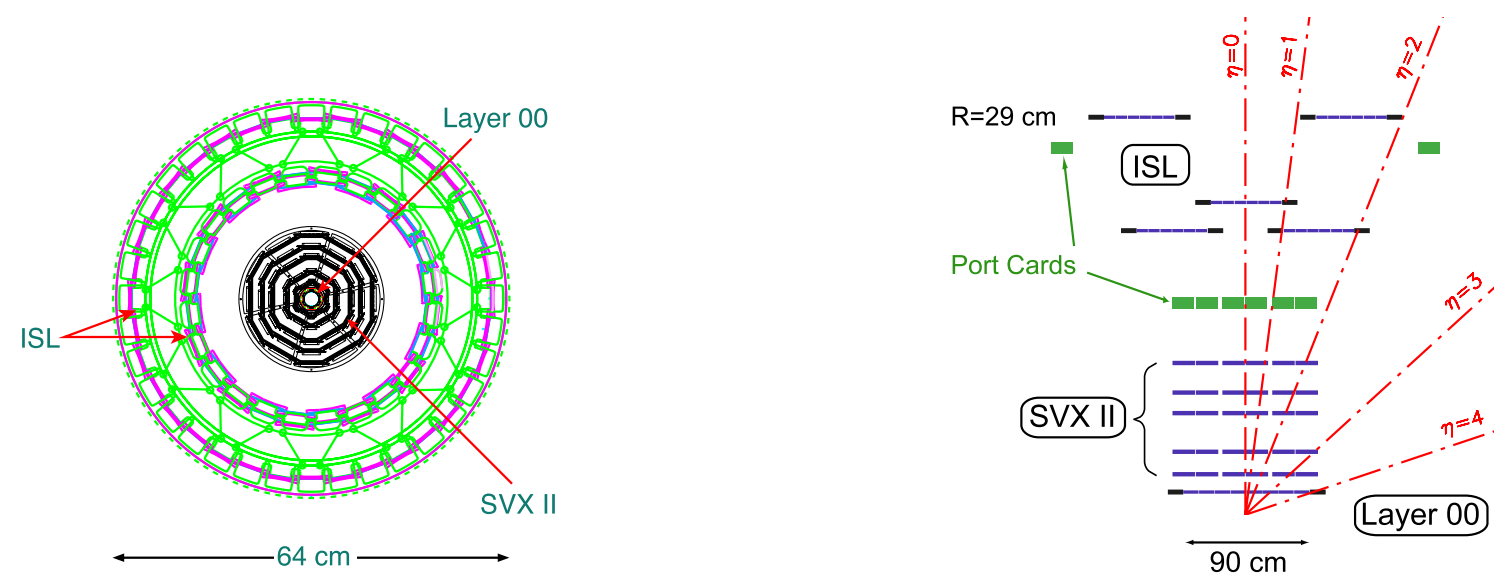

Figure 5.7. Schematic $r-\phi$ (left) and $r-z$ (right) views the Run II CDF silicon detector.

\section{Layer 00 (L00)}

L00 is a radiation tolerant, single-side $r-\phi$ layer, placed immediately outside the beam pipe at $\mathrm{R} \sim 1.5 \mathrm{~cm}$. Being so close to the interaction region L00 improves the impact parameter resolution from $40 \mu \mathrm{m}$ to $25 \mu \mathrm{m}$.

\section{Intermediate Silicon Layers (ISL)}

The Intermediate Silicon Layers were designed both to improve the tracking capabilities in the region $|\eta|<1$ where tracks are based on the SVX II and on the COT, and to allow silicon stand alone tracking outside the COT $\eta$ coverage. In the central $\eta$ region the ISL consists of a single layer of silicon placed at a radius of $22 \mathrm{~cm}$, while 
in the forward region $(1.0<|\eta|<2.0)$, where the COT coverage is incomplete or missing, it has two layers of silicon at radii of $20 \mathrm{~cm}$ and $28 \mathrm{~cm}$. A silicon sensor consists of double-sided micro-strip detectors with $r-\phi$ strips on one side and small angle stereo strips on the other. To reduce the number of channels the readout pitch is twice the strip pitch. The intermediate strips are not read out but they still contribute to the resolution through charge sharing. Two ladders with three sensors each are paired to form a module $\sim 55 \mathrm{~cm}$ long. The readout hybrids are not mounted on silicon like SVX II, but they are glued onto the edge of the mechanical support.

\section{Central Outer Tracker (COT)}

The COT detector is an open cell drift chamber located in a region between the radii of 40 and $138 \mathrm{~cm}$ from the beam pipe. It consists of eight super-layers which cover the $|\eta|<1$ region. Each super-layer groups 12 planes of sense wires alternated with layers of potential wires. To reconstruct tracks in three dimensions, four of the eight super-layers (axial) have wires along the axial direction, while the remaining four (stereo) have wires tilted of $\pm 3^{\circ}$ with respect to the axial direction; the super-layers are alternated starting with a stereo super-layer. To cope with the high luminosity and the event pile-up keeping the device occupancy to reasonable values, the cell size was reduced by a factor of four with respect to Run I. Moreover the chamber is filled with a mixture of Argon and Ethane in proportions of 50:50, which, with a drift velocity of $\sim 100 \mu \mathrm{m} / \mathrm{ns}$, ensure a faster response of the COT.

\subsubsection{Time of Flight Detector}

A Time Of Flight (TOF) detector dedicated to particle identification is located immediately outside the tracking system. It consists of 3 meter long scintillator bars covering the COT active volume. The $4 \mathrm{~cm}$ thickness of these bars is constrained by the limited space between the COT and the solenoid, while the $4 \mathrm{~cm}$ width has been determined by resolution and occupancy studies. A particle reaching the TOF detec- 
tor produces scintillation light which is collected by photo-multiplier tubes connected to both sides of each bar. The signal collected is used to determine the time interval between the production and the detection of the particle. This signal also gives a measurement of the $z$ coordinate of the particle in the TOF detector. The device is used to discriminate between protons, kaons and pions. With a time resolution of 100 ps, the system is able to provide 2 standard deviation separation between $\mathrm{K}^{ \pm}$and $\pi^{ \pm}$for momenta $\mathrm{p}<1.6 \mathrm{GeV} / c$, complementing the specific identification using the ionization energy loss $\mathrm{dE} / \mathrm{dx}$ measured by the COT.

\subsubsection{Calorimetry system}

The CDF calorimetry system, located immediately outside the solenoid, measures the energy and the direction of neutral and charged particles escaping the tracking region. The calorimeters are designed to absorb particles and degrade their energy through electromagnetic and hadronic interactions. They are organized into two subsystems: the inner electromagnetic and the outer hadronic section, optimized to identify electromagnetic and hadronic showers respectively. Both subsystems are segmented in towers projecting toward the center of the detector, providing spatial information on the shower direction. Each tower consists of layers of passive material alternated with scintillator tiles. Particles, gradually absorbed by the passive material, leave a signal in the scintillator which is read by wavelength shifters (WLS) and carried through light guide to photo-multiplier tubes.

\section{Central Calorimeter}

The Central Calorimeter consists of two halves joined at $\eta=0$ and covers the central region $|\eta|<1.1$. The Central Electromagnetic (CEM) subsystem is a sampling calorimeter made of lead sheets separated by polystyrene scintillator. Each half is organized in 24 wedges in $\phi$, sub-tending an angle of $15^{0}$. Each wedge is segmented into steps of $\Delta \eta=0.11$ in 10 projective towers. To increase the spatial resolution of 
the calorimeter two proportional chambers are embedded in each wedge of the CEM. The Central Electromagnetic Strip chamber (CES), placed 5.9 $X_{0}$ deep in the EM towers, consists of wires in the $r \phi$ plane and cathode strips in the $z$ direction. It measures the charge deposition in correspondence of the maximum electromagnetic shower development. The pulse-height and the three-dimensional spatial position of this signal improve the reconstruction of electromagnetic objects. The accurate measurement is obtained by comparing the signal to test beam templates and the goodness of the comparison is measured by a $\chi^{2}$ function. The CES helps to distinguish between electron and photons based on the accurate position measurement and the matching to a track; also, it discriminates $\pi^{0}$ and $\gamma$ based on the size of the transverse shower profile. The Central Pre-Radiator (CPR) wire chamber, located in

the space between the magnetic coil and CEM, acts as a shower Pre-sampler. This detector is also used to discriminate $\pi$ 's from $\gamma$ 's.

The Central and the End Wall Hadronic calorimeters, respectively CHA and WHA, are located behind the CEM. The WHA is intended to cover the gap between the central and the plug hadronic sections. The transverse segmentation of the hadronic calorimeter is designed to match the geometry of the CEM. Towers, made of acrylic scintillator between iron sheets, use a readout scheme similar to the one used in CEM. The CEM (CHA) energy resolution is $13.5 \% / \sqrt{E_{T}}+2 \%\left(75 \% / \sqrt{E_{T}}+3 \%\right)$.

\section{Plug calorimeter}

The plug calorimeter extends from $|\eta|>1.1$ to $|\eta|<3.64$. The new calorimeter, based on similar design as the central one, consists of a lead-scintillator electromagnetic section (PEM) followed by an iron-scintillator hadronic section (PHA). The electromagnetic calorimeter contains a Shower Maximum Detector (PES) placed at a radial depth of $\sim 6 X_{0}$. This device consists of 16 detector sectors each covering $45^{0}$ in $\phi$, with two layer of strips per sector. To keep the occupancy acceptable each sector is divided in two segments in pseudo-rapidity. In addition the first layer of the 




Figure 5.8. $r-z$ view of the plug calorimeter.

electromagnetic calorimeters can be read out separately and it acts as a pre-shower detector. The active elements of both calorimeters are the scintillating tiles, which are arranged in projective towers. Groups of 48 or 28 towers (for $|\eta|<2.11$ and $|\eta|>2.11$ ) are organized in 12 concentric $\eta$ sectors, in the 0.1 to $0.64 \eta$ range according to the increasing pseudo-rapidity. As in the central calorimeter the active elements are read out using wavelength shifter, optical cables, and the light is then routed from the tiles to the photo-multiplier tubes. Each photo-multiplier collects the light from the whole tower.

The PEM (PHA) resolution is $16 \% / \sqrt{E_{T}}+1 \%\left(74 \% / \sqrt{E_{T}}+4 \%\right)$.

\subsubsection{Muon system}

The outermost device surrounding the calorimeter is the muon system. It is organized into 4 subsystems covering different $\eta$ regions: Central Muon Detector (CMU), Central Muon Upgrade (CMP), Central Muon Extension (CMX) and Barrel 
Muon System (BMU) shown in Figure 5.9. The muon system allows triggering and identification of muons up to $|\eta|<1.5$.



Figure 5.9. Location of the muon upgrade components in azimuth $\phi$ and pseudo-rapidity $\eta$ for Run I. The gray cross-hatched region indicates the currently uninstrumented regions of CMP and CMX. Miniskirt and keystone, described in the text, are now instrumented.

\section{CMU, Central Muon Chambers}

The CMU chamber is located at the edge of CHA wedges and covers $|\eta|<0.6$. In particular for each CHA wedge per side, there are 3 modules in the CMU chamber. A module consists of four 1.25 degree wide stacks and each stack contains 8 cells arranged in 4 radial layers of 2 cells each. The cell contains one $50 \mu \mathrm{m}$ stainless steel 
sense wire. The 8 cells have a small $\phi$ offset between the first and the third, and the second and the forth layer. The wires of the first and the third (as the second and the forth) layers are connected in the readout. Each wire pair is instrumented with a TDC to measure the muon local $\phi$ and an ADC on each end to measure the muon local $z$ position via charge division. The chamber is operated in proportional mode.

\section{CMP, Central Muon Upgrade}

The CMP is the outermost sub-detector of CDF up to $|\eta|<0.6$. Since it is located behind the CMU chambers as shown in Figure 5.4, the CMP chambers have higher signal-to-background ratio and increase the trigger efficiency of the CMU/CMP combination. Unlike the other sub-detectors, CMP has the shape of a box. The reason is that it uses the magnet return yoke steel as absorbing steel. It consists of 1068 single-wire drift tube cells, arranged in 4 layers. There are 77 four layer units along the top, 65 along the bottom, 62 on the north wall, and 63 on the south wall. The chambers are run in proportional mode with a maximum drift time of $\sim 1.4 \mu \mathrm{s}$. and the signals are read by TDCs. A layer of scintillator counters (CSP) is installed on the outside surface of the wall drift chamber. Each counter covers two chambers in width and half the chamber length. In total, there are 216 scintillator used for collecting timing information.

\section{CMX, Central Muon Extension}

The CMX chamber consists of drift tubes (CMX) and scintillator counters (CSX) located at each end of the central detector covering $0.6<|\eta|<1.0$. At 55 degrees the CMX/CSX system covers the CMP. CMX is divided into two parts. The upper conical section covers the upper 270 degrees in $\phi$ (wedges 0-14 and 21-23). The lower 90 degrees in $\phi$ (wedges 15-20) is called the "miniskirt" and it has slightly different geometry due to the floor. The conical section contains 1632 cells, 864 on the west and 768 on the east side, divided into 18 wedges in $\phi$ covering 15 degrees each. On 
the east side, the top two wedges (5 and 6 ) are missing due to the cryogenic utilities for the solenoid (on the west side, these wedges are new for Run II and are called the "keystone"). The CMX drift tubes are arrayed as an extension of the central system. There are four layers of 12 tubes for each 15 degrees sector and successive layers are half-cell offset.

\section{BMU, Barrel Muon Chambers}

The BMU is located outside the magnet, in the forward region covering $\eta$ in the range 1.0 to 1.5 . Similarly to the other chambers it is a wire chamber operated in proportional mode. It consists of 1728 chambers total, 864 per toroid, arranged into 4 layers with 216 chambers per layer. Each chamber occupies 1.25 degrees in $\phi$. The bottom 90 degrees are uninstrumented.

\subsubsection{Trigger and data acquisition system}

The trigger systems have a crucial importance in hadron collider experiments. The collision rate, proportional to the large $\mathrm{p} \overline{\mathrm{p}}$ inclusive cross section, is in fact much higher than the rate data can be stored into tape. In particular at Run II the collision rate is of the order of $7 \mathrm{MHz}$, while the tape writing speed is limited to less than 70 events per second. The role of the trigger selection system is to effectively filter the interesting physics events among the large amount of background. The CDF trigger system has an architecture based on three decision levels. The rejection rate at each level is such that more sophisticated event processing is allowed at the next level with a minimum dead-time. The main characteristics of the three trigger levels are detailed in the following sections. 


\section{RUN II TRIGGER SYSTEM}
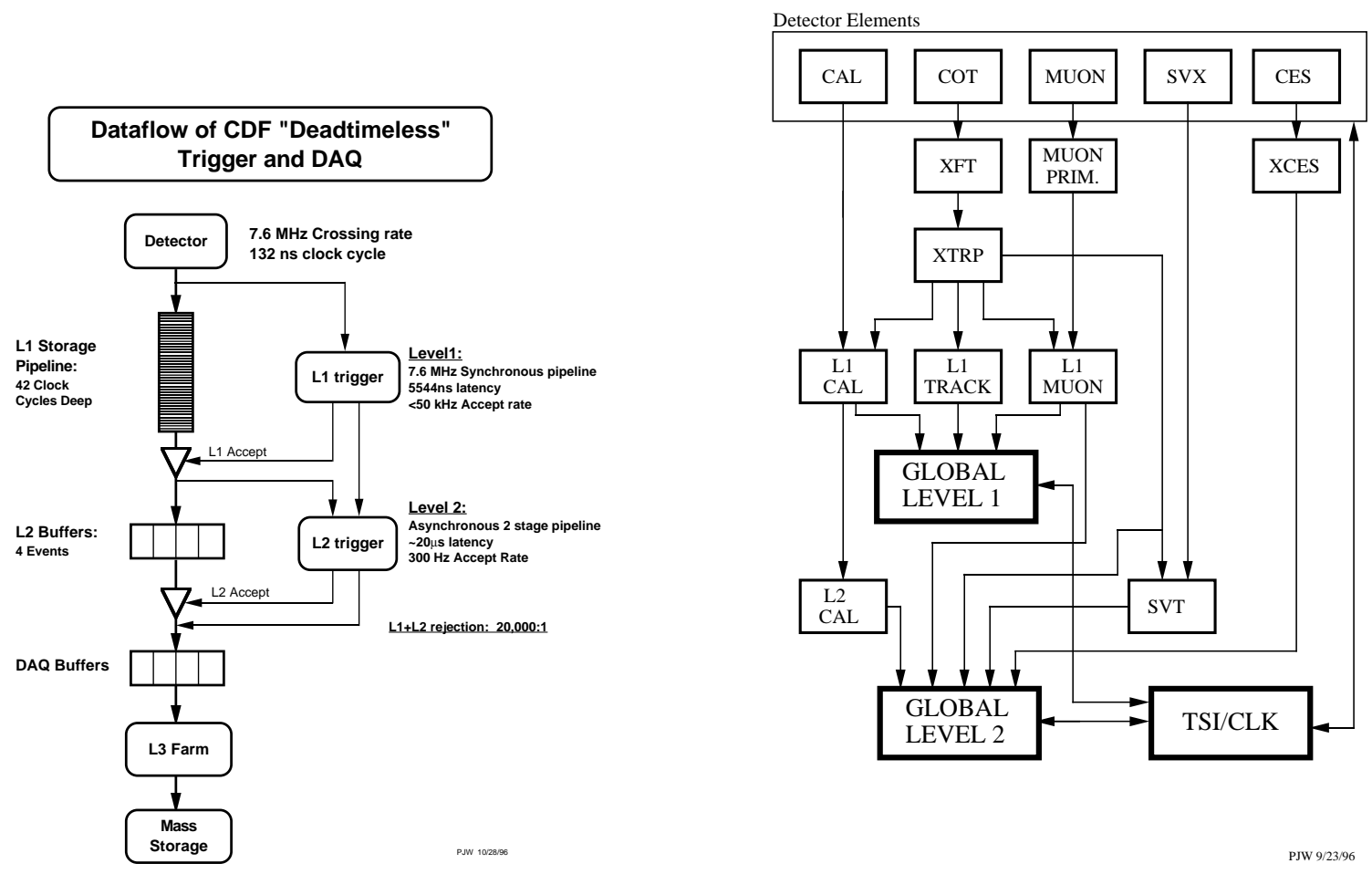

Figure 5.10. CDF pipelines and buffers trigger architecture (left). Block diagram of the Run II trigger system.

\section{Level 1 Trigger}

The Level 1 trigger (L1) selection is based on a number of physics object called primitives constructed from the detector signals. To guarantee enough time for transmission and processing of the information coming from the various sub-detectors, a L1 latency time of $5.5 \mu \mathrm{s}$ has been chosen. Each sub-detector is equipped with a local data buffering system able to accommodate the 42 events expected during this period. L1 primitives are constructed by dedicated hardware cards, designed to analyze signals from the calorimeters, tracking chambers and muon detectors. The most significant upgrade with respect to Run I is a new hardware track finding algorithm named extremely Fast Tracker (XFT). XFT analyzes the COT signals, returning the track $p_{T}$ and $\phi_{0}$ by means of $r \phi$ pattern recognition. These quantities are then used 
to better identify electron and muons, extrapolating and matching XFT tracks to calorimeter towers and to muon chambers. This task is performed by the Extrapolation Unit (XTRP), a custom designed trigger board. The calorimeter information used at L1, are the calorimeter towers grouped in pairs along $\eta$ denoted trigger towers. Using the electromagnetic and the total transverse energy of these trigger towers, electron/photon and jets primitives are built. Moreover using all the energy deposited in the trigger towers above $1 \mathrm{GeV}$, a crude value for the $\mathbb{F}_{T}$ is computed. The maximum L1 accepted rate is $\sim 20 \mathrm{kHz}$, while the typical one is about $12 \mathrm{kHz}$.

\section{Level 2 Trigger}

Events accepted by L1 are then processed by the Level 2 trigger (L2). At this level, the event information is collected into one of the four L2 buffers. When L2 is processing an event, the buffer where the event is stored is not accessible by L1; if all the four buffers are full the data can not be processed resulting in dead-time. To overcome this problem each L2 buffer is connected to a two-step pipeline each taking approximatively $10 \mu \mathrm{s}$. The information from the sub-detectors are first collected and then used to take the decision. The dead-time is less than $10 \%$ even at full L1 rate. At L2 all the L1 primitives are recalculated with higher precision. Moreover, to further reduce the accepted rate, the outcome of the trigger tower clustering can be used, as well as the information on the impact parameter provided by the Silicon Vertex Tracker (SVT). SVT is an hardware algorithm, implemented into dedicated processors, allowing CDF, for the first time in a hadron collider experiment, to trigger on secondary vertices. The typical L2 accepted rate is between 100 and $300 \mathrm{~Hz}$, depending on the luminosity.

\section{Level 3 Trigger}

The Level 3 (L3) trigger is structured into two steps. The information of events passing the L2 are structured into one data block, passed to the L3 (Linux) farms, 
where the whole event is reconstructed almost at analysis level. Variables such as global event observables, might not be calculated due to the long processing time required. Events passing L3 are monitored in real time in the CDF control room and stored on tape in Feynman Computer Center of Fermilab. They are organized in datasets [46], depending on the 3-level trigger path, defined as a unique combination of L1, L2 and L3 requirements.

\subsubsection{Triggers for data analysis}

In our analysis we explore the data sample collected with the high $p_{T}$ single muon triggers denoted CMUP18 and CMX18. The specific trigger requirements for CMUP18 are listed for completeness:

- Level 1. L1_CMUP6_PT4. Hits located in a CMU stack with arrival times within 124 ns of each other are matched to a CMP pattern and an XFT track with $p_{T}>4 \mathrm{GeV} / c$.

- Level 2. L2_AUTO_L1_CMUP6_PT4 and L2_TRK8_L1_CMUP6_PT4 The first path was used for the initial data taking where no track requirement is applied; later an XFT track with $p_{T}>8 \mathrm{GeV} / c$ is required.

- Level 3. L3_CMUP18 A reconstructed muon with $p_{T}>18 \mathrm{GeV} / c$ is required to have CMU and CMP stubs (within respectively 10 and $30 \mathrm{~cm}$ in the $\mathrm{r}-\phi$ plane from the extrapolated COT track).

and for $C M X 18$,

- Level 1. L1_CMX6_PT8. Hits located in a CMX stack with arrival times within 124 ns of each other are matched to a scintillator and an XFT track with $p_{T}>4 \mathrm{GeV} / c$.

- Level 2. L2_AUTO_L1_CMUP6_PT4 and L2_TRK8_L1_CMUP6_PT4. No additional requirements. 
- Level 3. L3_CMUP18 A reconstructed muon with $p_{T}>18 \mathrm{GeV} / c$ is required to have CMX stub (within $30 \mathrm{~cm}$ in the $\mathrm{r}-\phi$ plane from the extrapolated COT track)

Table 5.1 quotes the efficiency for the paths calculated in [47]. It is important to note that the trigger efficiency shows a time dependence due to detector performance variations, instantaneous luminosity changes and modification to the trigger path itself. These changes are properly taken into account in the analysis. The L1 and

Table 5.1

Trigger path efficiency (the uncertainty is statistical only)

\begin{tabular}{|c|c|}
\hline Path & Efficiency \\
\hline \hline CMUP18 & $89.77 \pm 0.5$ \\
CMX18 & $96.65 \pm 0.4$ \\
\hline
\end{tabular}

L2 trigger efficiency measurements have been cross checked in detail as shown in Appendix A.

\subsubsection{Luminosity measurement}

The Luminosity at CDF is measured both online and offline with the Cherenkov Luminosity Counters (CLC) using the inelastic p $\overline{\mathrm{p}}$ scattering. Two CLC modules are installed at small angles in the proton and antiproton directions, with rapidity coverage between 3.75 and 4.75. Each module consists of $48 \mathrm{~cm}$ long Cherenkov counters, filled with isobutane. The counters are arranged around the beam-pipe in three concentric layers. The online luminosity is recorded in real-time and takes into account multiple interactions automatically. Each CDF event contains both the online and offline luminosity information, the average instantaneous luminosity and the integrated luminosity. The uncertainty of the luminosity measurement is dominated by the uncertainty in the elastic $\mathrm{p} \overline{\mathrm{p}}$ cross section (4\%), the CLC acceptance 
and the related stability $(4.2 \%)$, together with uncertainties on beam losses, beam position and statistics. The total uncertainty is $\delta L / L \simeq 6 \%$ [48]. Figure 5.2.8 shows the luminosity delivered by the Tevatron from 2002 and the CDF detection efficiency in the same time period.



(a) Total delivered and stored luminosity as a function of time.

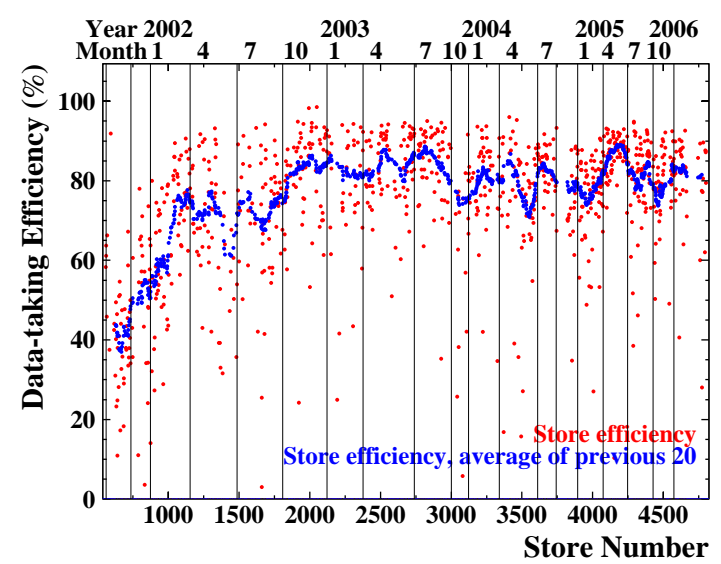

(b) CDF detection efficiency as a function of time.

\subsubsection{Collected luminosity for data analysis}

Our analysis is based on the data collected between March 2001 and August 2005. The Accelerator provided provided almost $1.3 \mathrm{fb}^{-1}$ of data. Nevertheless not all the delivered data are available for the physics analyses. Once the data are collected and stored on tape, each run is inspected for sub-detector failures. In fact, if any of the sub-detectors crucial for the analysis (for instance any of the muon chambers) encountered a hardware or software problem during the data taking, the data might be compromised. The quality of the data is secured by selecting only the data collected with properly functioning sub-detectors. Our sample contains $\mathcal{L}=745 \pm 45 \mathrm{pb}^{-1}$ of data which is reduced to $\mathcal{L}=680 \pm 41 \mathrm{pb}^{-1}$ if the silicon tracker is required to be operating [49]. 


\section{Event reconstruction}

At $\mathrm{CDF}$, the raw data recorded after the $\mathrm{L} 3$ decision is made, are calibrated and processed by reconstruction software. It constructs basic objects such as vertices, tracks, muons, jets and electromagnetic clusters that are needed for particle identification. The latter consists in comparing the properties of a given object against a priori criteria, called identification criteria, defined from the known features of tracks, muons, electrons, etc. For instance the ID criteria helps to distinguish between real muons and pions reaching the muon chambers. They are also designed to select candidates most likely originating from the decay of charginos and neutralinos. To insure sensitivity to new physics, the ID should have high efficiency and purity. Nevertheless even a sample of candidates passing the ID criteria might be contaminated by misreconstructed objects, as discussed in Section 7.4.

\subsection{Tracks}

The pattern recognition utilizes different pieces of information depending on the $\eta$ region of the detector. In the so called "central" region of CDF $(|\eta|<1.0)$, the tracking algorithm is based either on COT only hits (COT Tracking) or on a combination of COT hits and Silicon clusters (Outside-In Tracking) [50] [51].

- COT Tracking. The algorithm that reconstructs the track of a particle traversing the COT is hit based. First it converts the TDC output into hit position. Once the position of the COT hits candidate is known, the tracking algorithm searches for hits in 3 consecutive wires and fits them to a straight line. During a second iteration, hits within $1 \mathrm{~mm}$ (corresponding to $20 \mathrm{~ns}$ if the drift velocity is $55 \mu \mathrm{m} / \mathrm{ns}$ ) are added and the parameters of the line segment recalculated. The resulting segments are the building blocks of the "segment linking" and 
the seeds for the "histogram linking". The "segment linking" assembles the segments into $r-\phi$ tracks. Hits in common to two tracks are assigned to the track with the largest number of hits. The "histogram tracking" improves the reconstruction efficiency since it recovers missed hits with a "telescope based" strategy. Based on a single axial segment and the beam position, the algorithm draws several circular trajectories and matches hits found in a band of $1 \mathrm{~cm}$. A $200 \mu \mathrm{m}$ binned histogram is filled with the radius of each hit. If one bin, corresponding to a given radius, has more than 10 hits, a track is reconstructed. The histogram tracking adds other hits if located within $750 \mu \mathrm{m}$ and performs a re-fit. Only tracks with more than 20 hits are called "CDF tracks". Once the tracks are reconstructed in $r-\phi$ the stereo information is attached. The algorithm starts from the outer stereo layer and matches stereo segments to tracks. At each step, a re-fit of the track provides the $z$ and angular information. Finally the track parameters are modified for material effects, variations in the magnetic field value, energy loss, etc.

- Outside-In Tracking. The OI tracking extrapolates back each COT track to the Silicon and adds Silicon hits by a progressive fit. The fit uses hits located within 4 standard deviations (based on the track error matrix) from the seed track. A new track candidate is then extrapolated to the next Silicon layer. The candidate track with the largest number of Silicon hits is selected as the Silicon track for the given COT track.

If the track falls in the forward region $(|\eta|>1.0)$, the track reconstruction uses the Silicon hits, following two diffent approaches, the Silicon Stand-alone and the Phoenix Tracking. We use Silicon Stand-alone (Phoenix) tracking for track $p_{T}<15 \mathrm{GeV} / c$ $\left(p_{T}>15 \mathrm{GeV} / c\right)$. The Silicon Stand-alone tracking reconstructs tracksks which do not traverse the entire COT and requires $23 \mathrm{D}$ hits and $2 \mathrm{r}-\phi$ hits (the track should qualify as IO track ${ }^{1}$ ). The Phoenix algorithm loops over the electromagnetic clusters

${ }^{1}$ The track must have at least 2 axial/stereo COT SL if it passes through the entire COT; it has to have less than $15 \%$ COT hits in common with other tracks. 
and builds hypothetical tracks used as seed for the OI pattern recognition.

The COT track reconstruction efficiency is $99 \%$ for track $p_{T}$ larger than $1.5 \mathrm{GeV}$ and decreases to $95 \%$ for $p_{T}$ in the range 0.5 to $1.5 \mathrm{GeV}$. The Silicon efficiency is shown in Figure 6.1.

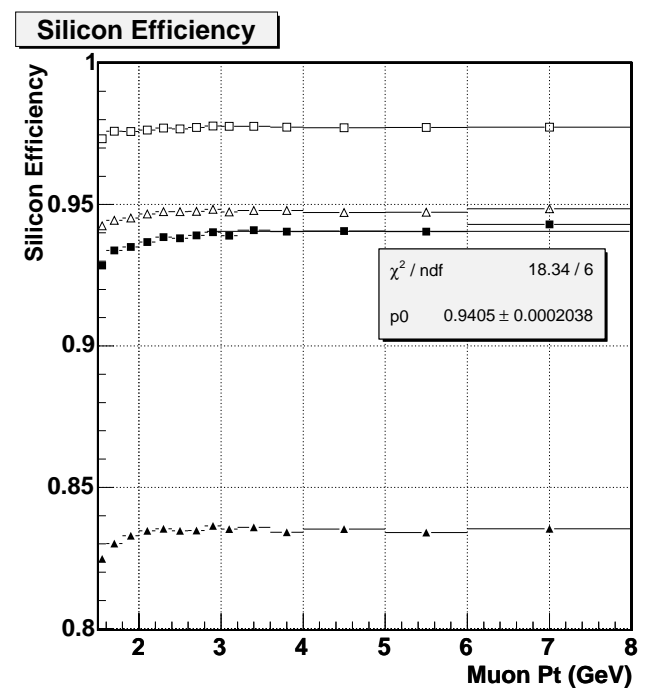

Figure 6.1. Silicon efficiency vs $p_{T}$. Open squares: COT track intersects three active $r-\phi$ layers of SVXII. Open triangles: COT track intersects three active stereo layers of SVXII. Closed squares: Silicon $r-\phi$ hits found in three layers of SVXII. Closed triangles: Silicon stereo hits found in three layers of SVXII

The track helix is parametrized in terms of 5 parameters:

- curvature c proportional to the transverse momentum as

$$
c=2 \times r=\left|p_{T} /(q e B)\right|
$$

where $r$ is the helix radius and B is the magnitude of the magnetic field;

- $\cot \theta$ where $\theta$ is the polar angle;

- impact parameter $d_{0}$, defined as the minimum distance from the interaction point in the transverse plane; 
- $Z_{0}$ the z-coordinate of the track at the minimum distance from the interaction point;

- $\phi_{0}$ the azimuthal angle of the track at the minimum distance from the interaction point in the transverse plane;

The variables used to identify the tracks of charged particles developed for our analysis are the following.

- A track is required to have more than 2 (1) axial (stereo) COT segments with at least 5 hits each. If the track is matched to a stub-less muon (Section 6.3) the number of stereo segments must be larger than 2 as well. The probability of muon misidentification is higher for stub-less muons than for stub muons; a tighter requirement on track quality helps in suppressing misreconstructed muon.

- The goodness of the fit to the hits during the track reconstruction is measured by the the $\chi^{2} /$ ndof and is required to be smaller than 2.75 (or 2.3 depending on the data taking period). This selection is applied only to tracks identified as muons in order to reject events where a pion or kaon decaying "in flight" is reconstructed as a high $p_{T}$ muon.

- The impact parameter of the track $d_{0}$ should be within $0.02 \mathrm{~cm}$ from the primary vertex if the COT track is matched to a track in the Silicon Tracker; otherwise, $d_{0}$ is required to be less than $0.2 \mathrm{~cm}$. Tracks from cosmic rays and non-prompt decays are likely to fail this requirement.

- The track $Z_{0}$ must be within $60 \mathrm{~cm}$ from the center of the detector. The track therefore originates from the luminous region and falls in the tracking volume of CDF.

- The track is "beam constrained" in that the track parameters are recalculated after requiring that the track originates from the beam spot. 
- An angular dependent curvature correction is needed to take into account the misalignment in the COT [52].

The distributions of the track identification variables are shown in Figure 6.2.

\subsection{Primary vertex}

The $x$ and $y$ coordinates of the primary interaction point are measured for each Tevatron store, while the $z$ coordinate is measured event by event. The vertex reconstruction algorithm is seed driven [53]. It first generates a list of vertex candidates based on Silicon SAS and COT stereo information; then it assign each track in the event a quality value depending on the number of COT segments and Silicon hits. For instance the COT highest $p_{T}$ track is by definition a quality 12 track, whereas a track with at least 2 axial and 2 stereo COT segments and at least 4 axial and 3 stereo SAS Silicon hits is a quality 2 track. Each track must have $d_{0}<1 \mathrm{~cm}$. The quality of the vertex, defined as the sum of the track qualities, is required to be larger or equal to 12 . If there is no well reconstructed high $p_{T}$ track in the event, the vertex finder checks if there are tracks with lower quality and similar $z_{0}$ coordinate. In this case the vertex location is calculated as the weighted average of the selected tracks. If two vertex candidates are closer than $3 \mathrm{~cm}$, they are merged into a single vertex. If there are more than 1 vertices, the primary interaction is associated to the vertex with the highest total transverse energy. We usually require the vertex to fall within $60 \mathrm{~cm}$ from the center of the detector. The vertex reconstruction efficiency is $95.8 \pm 0.2 \%[54]$.

\subsection{Muons}

Similarly to the other algorithms, the muon reconstruction proceeds in steps, starting from the signal in the detector up to the muon object [55]. First the drift time is converted into a drift distance; in particular, the CMP and CMX chambers provide the position perpendicular to the sense wire only, whereas the CMU chamber 


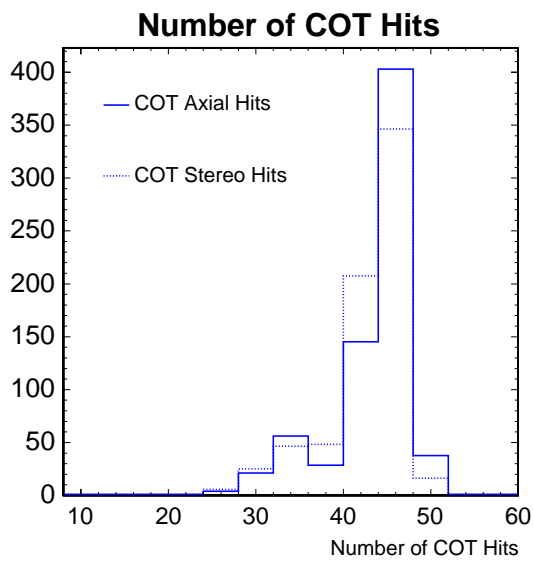

(a) Number of COT axial and stereo hits.

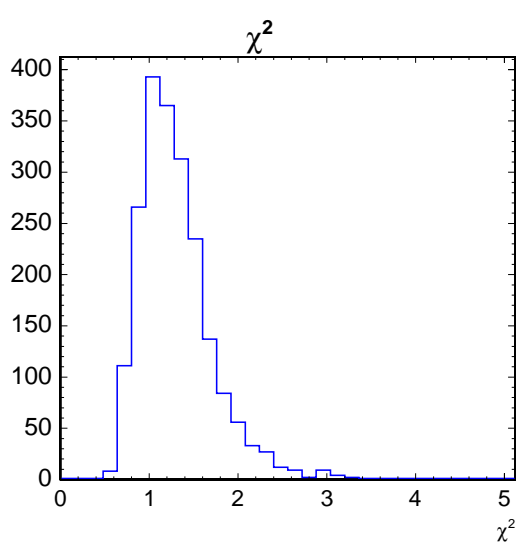

(c) Track $\chi^{2}$.

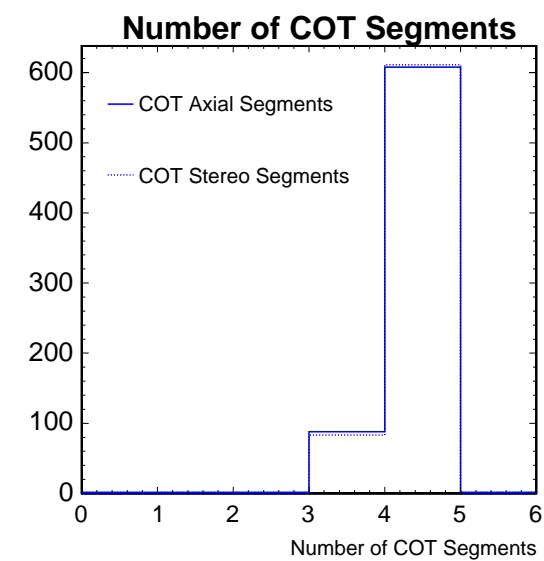

(b) Number of COT axial and stereo segments.

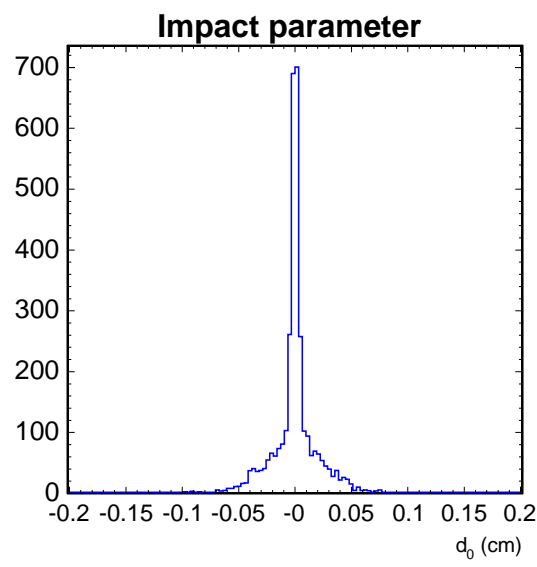

(d) Impact parameter.

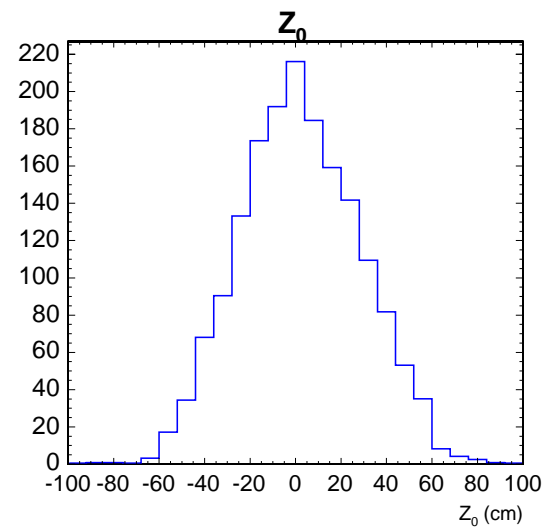

(e) Z coordinate.

Figure 6.2. Identification variables of tracks. 
can also measure the position of the muon along the sense wire. Once the hit collection is obtained, the algorithm selects candidate regions depending on the number of hits ${ }^{2}$. If a region is selected then the so called "stub finder" examines the pairs of hits on Layer 0 and Layer 2 in the stack and accepts those located within $7.5(12) \mathrm{cm}$ in the CMU (CMP) chamber. The parameters of the resulting segment are needed to extrapolate the line to Layer 1 and Layer 3 and attach additional hits. If the segment is matched to either 3 or 4 hits, it becomes a so called "stub". The procedure is repeated using Layer 1 and Layer 3 as seeds ${ }^{3}$. The stub linking algorithm tries to match the stubs to extrapolated tracks with $p_{T}>1.3 \mathrm{GeV},\left|d_{0}\right|<6 \mathrm{~cm},\left|Z_{0}\right|<200 \mathrm{~cm}$ and more than 10 COT axial hits, by mini-mixing a fit $\chi^{2}$. A track can be associated to more than one stub in different muon systems but each stub cannot belong to more than one track. Once the stub is matched to the track, the calorimeter information is retrieved: the towers crossed by the muons are identified by extrapolating a muon track to the electromagnetic and hadronic calorimeter. In particular a muon with both CMU and CMP stubs is called CMUP muon and stub-less muons are referred to as CMIO (Central Minimum Ionizing Object). The reconstruction efficiency measured in [47] depends on the muon chamber, as shown in Table 6.1 and it may vary in different data taking periods.

Similarly to the tracks, the reconstructed muons are identified by analysis driven criteria.

- The transverse momentum of the track (selected as in Section 6.1) is requested to be larger than $5 \mathrm{GeV} / c(10 \mathrm{GeV} / c)$ for stubbed (stub-less) muon. We do not expect significant contributions of muons with lower transverse momentum from the decay of charginos and neutralinos. A tighter cut is applied to stub-less

\footnotetext{
${ }^{2}$ If the number of hits is less than 3 , the signal is considered noise and if the hits are more than 25 , the region is discarded

${ }^{3}$ Once the candidate stubs are constructed, the stub parameters are re-evaluated to take into account the drift model. In the CMU chambers the xy fit is performed by the standard linear least squares method, while the xz residual of the fit falls between the hit drift distances and the drift distance extrapolated from the stub parameters. In the CMX chambers the hit and stub finding procedure is similar.
} 
Table 6.1

Muon reconstruction efficiency measured in data and in simulated data (statistical uncertainty only).

\begin{tabular}{|c|c|c|}
\hline Type & Efficiency (data) & Efficiency (MC) \\
\hline \hline CMUP & $91.98 \pm 0.37 \%$ & $90.95 \pm 0.35 \%$ \\
CMX & $98.84 \pm 0.22 \%$ & $96.04 \pm 0.31 \%$ \\
\hline
\end{tabular}

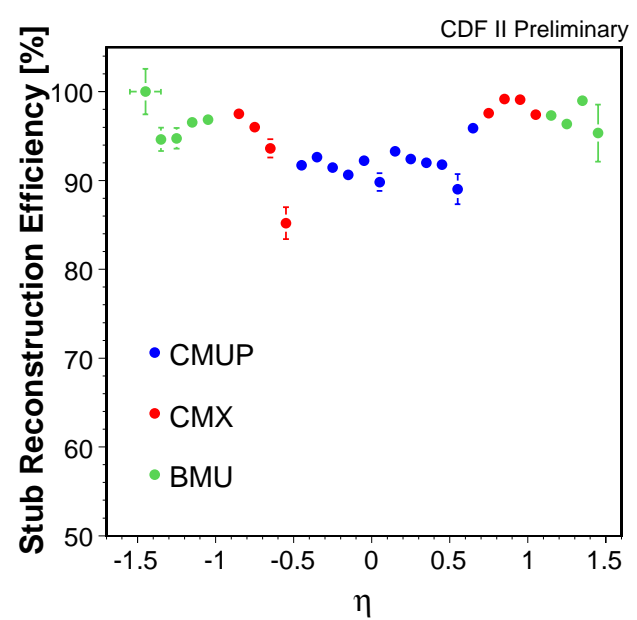

Figure 6.3. Muon reconstruction efficiency as a function of $\eta$.

muons to reduce the contamination from misidentified muons, non negligible for muons with $p_{T}<20 \mathrm{GeV} / c$.

- The maximum electromagnetic energy deposited by the muons is set to $2 \mathrm{GeV}$ whereas a sliding selection criteria is required for the the hadronic energy $E_{H a d}<3.5+p_{T} / 8$. Muons are not expected to interact with the material since they are minimum ionizing particles (MIP).

- The total energy in the calorimeter is required to be larger than $0.1 \mathrm{GeV}$ if the muon track is not matched to a stub. This prevents us from misidentifying a track pointing to a calorimeter crack as a muon. 
- The matching between the stub and the track is described by two variables, the local distance between the extrapolated track and the stub $(\Delta X)$, and a $\chi^{2}$ variable which takes into account the error on $\delta x$ and $\delta \phi$. If the muon $p_{T}$ is larger than $20 \mathrm{GeV} / c$, the quality of the stub-matching is set by $\Delta X(\Delta X<7$, 5, 6 for CMU, CMP, CMX). For soft muons, the $\chi^{2}$ is utilized $\left(\chi^{2}<9\right)$ being Gaussian distributed and less affected by multiple scattering as the error due to the multiple scattering is taken into account.

- The COT exit radius defined as

$$
\rho_{C O T}=\frac{\eta}{|\eta| \times \frac{z_{C O T}-Z_{0}}{\tan \left(\frac{\pi}{2}-\theta\right)}}
$$

where $z_{C O T}=155 \mathrm{~cm}$ corresponds to the COT endpoint, is required to be larger than $140 \mathrm{~cm}$ for CMX muons with $p_{T}$ larger than $20 \mathrm{GeV} / c$. This requirement insures that a CMX muon can trigger at high efficiency ${ }^{4}$.

- Geometrical constraints on the position of the muon stub are applied to avoid edge effects. The track is extrapolated to CMU, CMP or CMX and the distance from the projection to the edge of the chamber is measured, in both the $\mathrm{x}$ direction (drift distance) and perpendicular to that. A negative value of the fiducial distance means that the track is projected inside the chamber ${ }^{5}$.

- The isolation is defined as the sum of the energy in the calorimeter towers within 0.4 from the muon track in the $r \phi$ space. The requirement depends on the transverse momentum of the muon to take into account the energy deposit in the calorimeter increasing with the muon $p_{T}$. If the muon $p_{T}$ is less than 20 $\mathrm{GeV} / c$, the maximum energy in the cone is set to $2 \mathrm{GeV}$; otherwise it scales with the $p_{T}$ as $0.1 \times p_{T}$.

- Energetic cosmic rays traverse the detector depositing hits in the muon chambers and the COT and they are likely to be reconstructed as high $p_{T}$ muons.

\footnotetext{
${ }^{4}$ The XFT trigger requires at least 10 hits for each of the four axial COT SL.

${ }^{5}$ We require that muons $x_{\text {fid }}<0 \mathrm{~cm}$ and $z_{f i d}<-3 \mathrm{~cm}$.
} 
In a large fraction of events, the cosmic ray firing the trigger is detected out of coincidence with the collision. Nevertheless a fraction of cosmic events are recorded in coincidence and need to be excluded from the analysis. The cosmic ray tagging algorithm benefits from timing information provided by the TOF detector and from the COT hit information. First the hits of the seed track associated with the candidate muon are re-fitted with the five free parameters and a floating additional variable representing the production time $t_{0}$. Based on the best fit values, the track is tagged as incoming or outgoing. The new track is used to seek for hits on the opposite side of the COT. If enough hits are found and a new fit matches all hits to a single track, the event is tagged as cosmic event [56] [57].

The distributions of the muon identification variables are shown in Figure 6.4. For sake of convenience we will call high (medium) $p_{T}$ muons, those muons with $p_{T}$ larger than 20 (5) GeV/c. The criteria applied to identify muons are summarized in Table 6.2 and Table 6.3 shows the identification efficiency. The measurement of the ID efficiency for medium $p_{T}$ muons is described in Appendix B for a given data taking period.

Table 6.2

Muon Identification selection criteria.

\begin{tabular}{|c|c|c|c|}
\hline ID Cut & High $\mathrm{p}_{T} \mu$ & Medium $\mathrm{p}_{T} \mu$ & CMIO \\
\hline$p_{T}(\mathrm{GeV} / c)$ & $p_{T}>20$ & $5<p_{T}<20$ & $>10 \mathrm{GeV}$ \\
\hline$\eta$ & $<1.0$ & same & - \\
\hline $\mathrm{Z}_{0}(\mathrm{~cm})$ & $<60 \mathrm{~cm}$ & same & same \\
\hline Fiduciality & CMU-CMP or CMX & same & Not Fiducial to CMU-CMP, CMX \\
\hline CMU,CMP,CMX Stub Matching & $\Delta X<7,5,6 \mathrm{~cm}$ & $\Delta X<7,5,6 \mathrm{~cm}$ or $\chi^{2}<9$ & - \\
\hline Track $\chi^{2}$ & $<2.5$ & same & same \\
\hline $\mathrm{d}_{0}(\mathrm{~cm})$ & $<0.02(0.2)$ Si Hits(no Si Hits) & same & same \\
\hline COT Axial 5-Hit Segments & $>2$ & same & same \\
\hline COT Stereo 5-Hit Segments & $>1$ & same & $>2$ \\
\hline Hadronic Energy $(\mathrm{GeV})$ & $<6+\left(\max \left(0,(\mathrm{p}-100)^{*} 0.028\right)\right)$ & $3.5+p_{T} / 8$ & as for stub muons \\
\hline Electromagnetic Energy $(\mathrm{GeV})$ & $<2+(0,(\mathrm{p}-100) * 0.0115)$ & 2 & as for stub muons \\
\hline Total energy $(\mathrm{GeV})$ & - & - & $>0.1$ \\
\hline Isolation & $E_{0.4} / p_{T}<0.1$ & same or $E_{0.4}<2 \mathrm{GeV}$ & $E_{0.4} / p_{T}<0.1$ \\
\hline
\end{tabular}




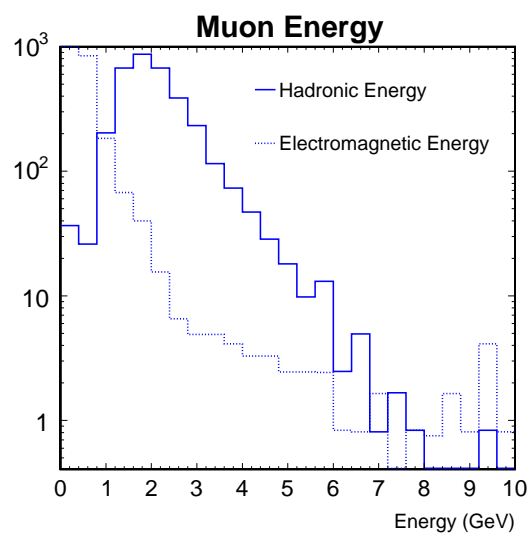

(a) Muon energy.

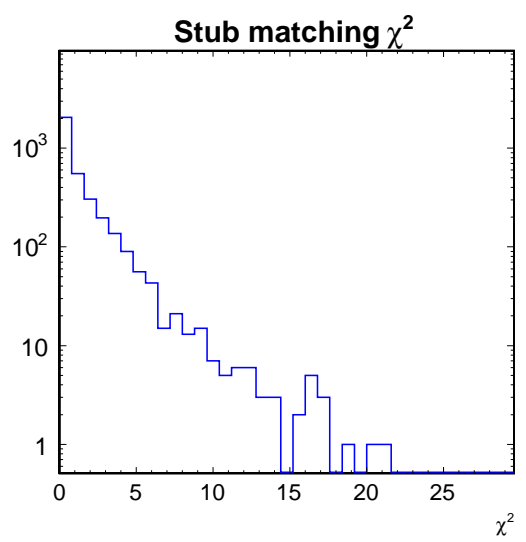

(c) Stub matching for low $p_{T}$ muons.

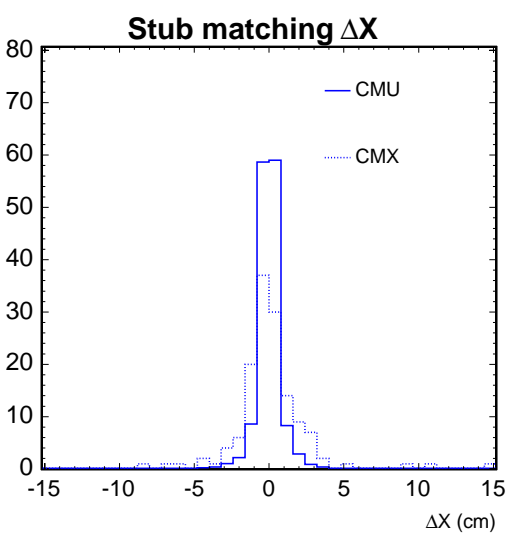

(b) Stub matching for high $p_{T} \mathrm{CMU}$ and CMX muons.

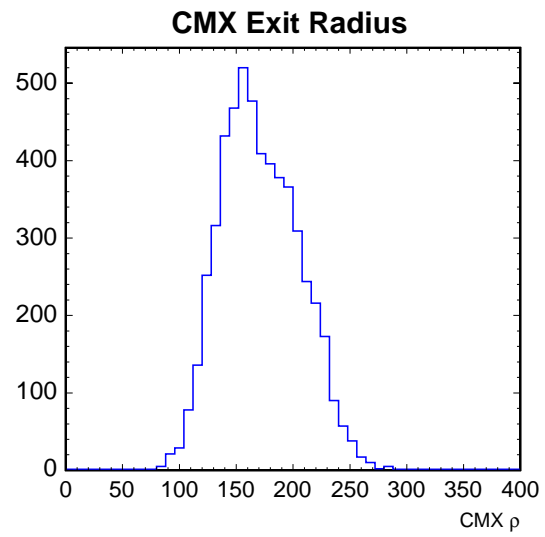

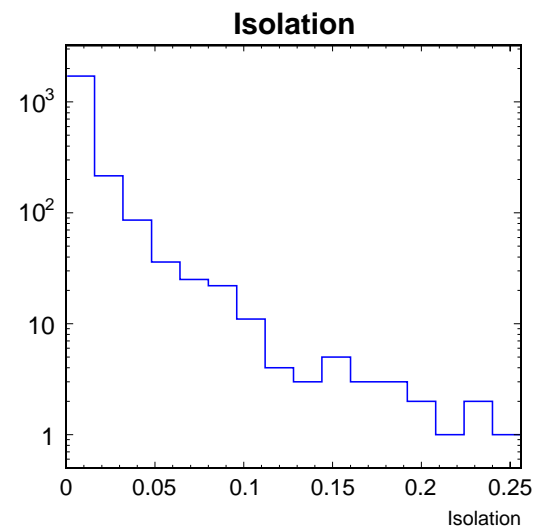

(e) Isolation.

Figure 6.4. Identification variables of muons. 
Table 6.3

High $p_{T}$ Muon identification efficiency (statistical uncertainty only).

\begin{tabular}{|c|c|c|}
\hline Type & Efficiency (data) & Efficiency (MC) \\
\hline \hline CMUP & $90.89 \pm 0.41 \%$ & $92.38 \pm 0.07 \%$ \\
CMX & $93.42 \pm 0.51 \%$ & $92.41 \pm 0.11 \%$ \\
CMIO & $88.12 \pm 0.98 \%$ & $82.95 \pm 0.24 \%$ \\
\hline
\end{tabular}

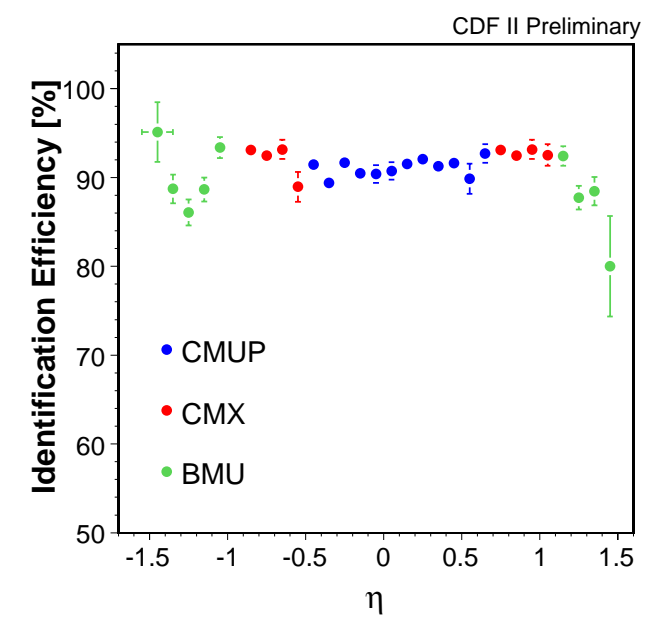

Figure 6.5. High $p_{T}$ muon identification efficiency as a function of $\eta$.

Table 6.4

Medium $p_{T}$ Muon identification efficiency with tight isolation requirement as measured in [58] (statistical uncertainty only).

\begin{tabular}{|c|c|c|}
\hline Type & ID Efficiency (data) & ID Efficiency (MC) \\
\hline \hline CMUP & $71.4 \pm 1.0 \%$ & $82.14 \pm 0.13 \%$ \\
CMX & $71.8 \pm 1.6 \%$ & $82.67 \pm 0.18 \%$ \\
\hline
\end{tabular}

\subsection{Electrons}

The reconstruction of central electrons is based on the track reconstruction and its matching to a cluster in the central electromagnetic calorimeter (CEM) [59]. The EM 
Table 6.5

Medium $p_{T}$ Muon identification efficiency with loose isolation requirement as measured in [58] (statistical uncertainty only).

\begin{tabular}{|c|c|c|}
\hline Type & ID Efficiency (data) & ID Efficiency (MC) \\
\hline \hline CMUP & $81.7 \pm 1.0 \%$ & $89.46 \pm 0.11 \%$ \\
CMX & $85.0 \pm 1.8 \%$ & $89.60 \pm 0.15 \%$ \\
\hline
\end{tabular}

clustering first applies a tower to tower calibration and sorts the towers in decreasing $E_{T}$. If the tower energy is larger than $0.2 \mathrm{GeV}$, the tower is utilized as a seed tower and combined to neighboring towers with energy larger than $0.1 \mathrm{GeV}$. The towers considered in the clustering procedure must be in the same detector ${ }^{6}$. This defines the maximum size of the EM cluster to be 3 towers in the polar direction $(\Delta \eta=$ $0.3)$ and 1 tower in the azimuthal direction $\left(\Delta \phi=15^{\circ}\right)$. Besides the energy, the electron reconstruction relies on the accurate determination of the position of the electron candidate provided by the CES. For a given view up to 11 channels are grouped together if the energy is above a threshold. The profile is then compared to profile templates of a single particle test beam. The centroid of the shower max cluster defines the location of the EM cluster. The electromagnetic object needs to be matched to a COT based track. Each track candidate is extrapolated to the CES plane and matched to the EM cluster. If more than one track satisfies the matching, the highest $p_{T}$ is retained.

Plug electrons are identified by clusters in the EM component of the plug associated with energy clusters in the PES. A similar reconstruction to the one described for the central region is performed. The electron identification depends on the $\eta$ coordinate. Furthermore we differentiate between tight and loose requirements, as needed in the analysis later. The physical quantities utilized for central electrons identification are the following.

${ }^{6}$ In the particular case of the CEM, they should also be within the same wedge as the seed tower. 
- The transverse energy of the central electromagnetic cluster and the transverse momentum of the matched track are required to be larger than 8 (5) GeV and 4 $\mathrm{GeV} / \mathrm{c}$ respectively for tight (loose) central electrons. The energy of the electron candidate is subject to a correction to take into account detector effects ${ }^{7}$.

- The EM shower is subject to fiduciality requirements.

- The ratio of the hadronic energy over the electromagnetic energy of the shower measures the leakage of energy from the EM calorimeter into the HAD calorimeter and it is primarily designed to distinguish electrons from jets. The ratio is required to be less than 0.00045 and it is modified to account for larger leakages for higher energy electrons as $E_{H A D}<0.00045 \times E_{E M}+0.055$.

- The isolation is defined similarly to muons but corrected for leakage of energy in neighboring towers as $E_{0.4}-E_{L e a k}<E_{T} \times 0.1$. The fractional cut is applied independently from the $p_{T}$ of the electron.

- A $\chi^{2}$ variable is used to compare the CES shape to templates of pulse height in z. A small value ensures that the candidate is consistent with an electron.

- The ratio of the energy over the momentum helps to identify if an electron undergoes bremsstrahlung. When the electron radiates a photon the momentum of the electron track decreases but the total energy assigned to the electron does not since the photon is often collimated with the electron and is detected in the same tower as the electron. We expect E/p to have a tail below 1 since the high $p_{T}$ electrons have large bremsstrahlung probability. A small E/p ratio may also indicate a mismeasurement of the electron energy.

\footnotetext{
${ }^{7}$ The resolution is affected by three effects. The first is the variation of the calorimeter response as a function of the local $\mathrm{x}$ and $\mathrm{z}$ coordinate. The tower to tower calibration is time dependent. The energy scale has to be measured. In particular, EM showers close to the edge of the wedge has lower response than the ones in the center. The so called "face" correction was determined during test beams. The tower to tower calibration is corrected using the data to calculate the average $\mathrm{E} / \mathrm{p}$ in a given tower as a function of time. The overall scaling is found using $\mathrm{Z}$ events.
} 
- The electron energy typically leaks in neighboring $\eta$ wedges of the electromagnetic calorimeter. We define a shower profile as

$$
L_{\text {share }}=0.14 \times \sum_{n} \frac{E_{n}-E_{n}^{e x p}}{\sqrt{0.14^{2} \times E_{\text {cluster }}+\left(\Delta E_{n}^{e x p}\right)^{2}}}
$$

where $\mathrm{n}$ is the index of up to 2 adjacent towers with energy $E_{n}$ at a given $\phi$ position; $E_{n}^{\exp }$ is the expected energy calculated from the projection of the electron track, $\Delta E_{n}^{e x p}$ is the associated uncertainty; finally $E_{\text {cluster }}$ is the energy of the seed cluster.

- The CES provides an accurate measurement of the $x$ and $z$ coordinate of the cluster. The difference between the cluster position and the extrapolation of the track is denoted by $\Delta X$ and $\Delta Z$, required to be in the range $-3.0<q \cdot \Delta X<$ 1.5 where $q$ is the electron charge, and $\Delta Z$ less than $3 \mathrm{~cm}$. A tight matching rejects hadrons misreconstructed as electrons.

In addition to the variables described above, the forward electrons are checked for number of clusters and towers in the PEM and PES respectively and strips in the PES. In particular, in the PES the highest energy strip is the seed strip. PES clusters have 9 strips centered around it. A variable called $P E S_{5 / 9}$ is defined as the energy of the 5 central strips divided by the total energy of the 9 strips. It helps to distinguish electrons or photons with wide shower from $\pi$ s with narrower showers.

The distributions of the electron identification variables are shown in Figure 6.6. The values required for all electron types are listed in Table 6.6 and the efficiency measured in [60], [61], [62] in Table 6.7. Beyond applying the identification criteria, we also require that electrons do not originate from photons converting in the material. The efficiency of the conversion tagging algorithm differs in data and in Monte Carlo and needs to be corrected for this effect. The details of the measurement of the conversion tagging efficiency are presented in Appendix C. 


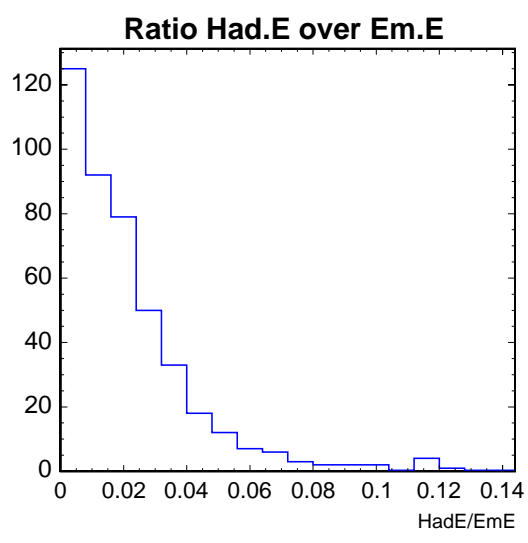

(a) Ratio of the hadronic energy over the electromagnetic energy.

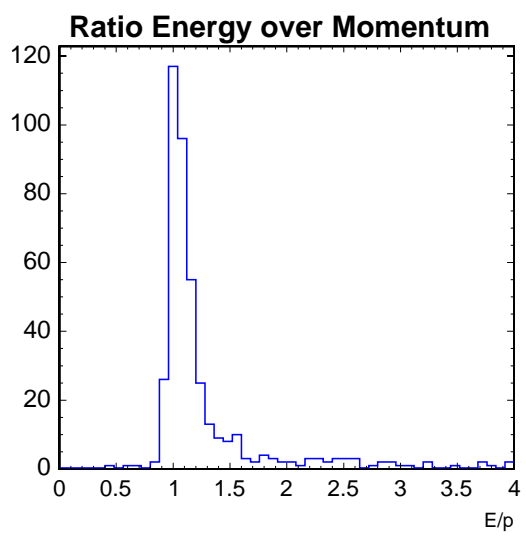

(c) Ratio of the energy over the momentum.

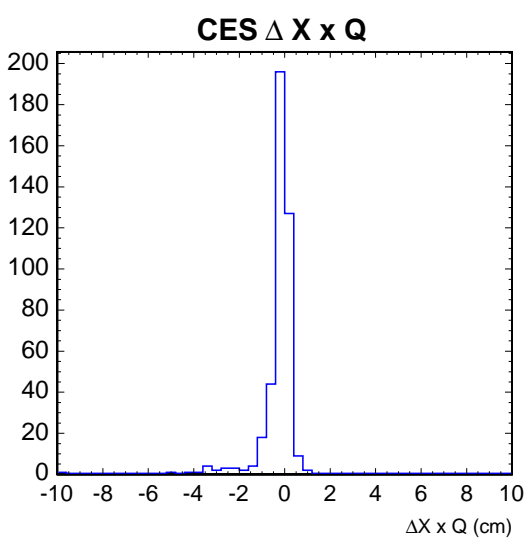

(e) CES $\Delta X \times Q$

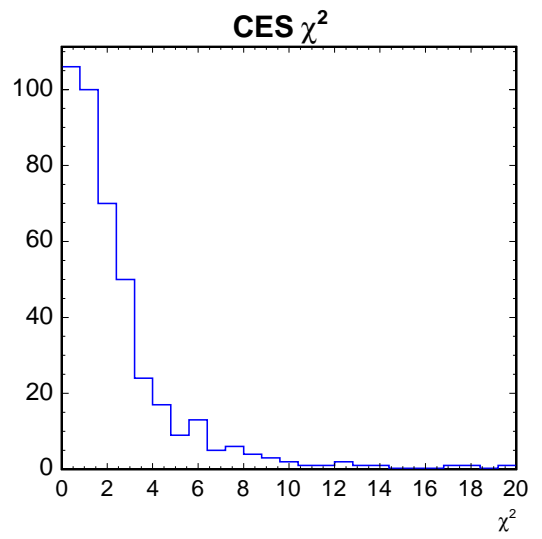

(b) $\operatorname{CES} \chi^{2}$.



(d) Lateral profile.

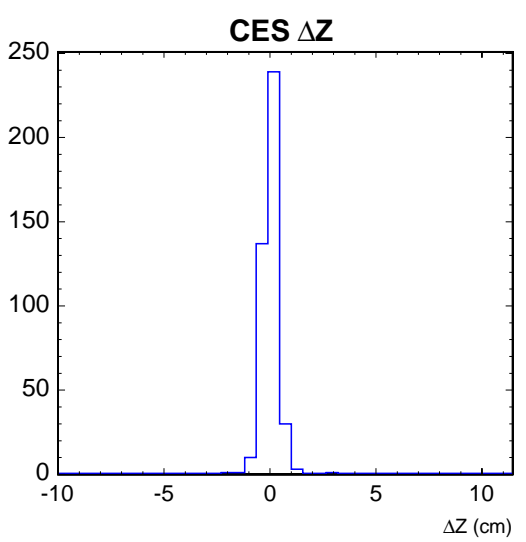

(f) $\operatorname{CES} \Delta Z$

Figure 6.6. Identification variables of electrons. 
Table 6.6

Identification selection criteria applied to electrons

\begin{tabular}{|c|c|c|c|}
\hline Central Tight $e$ (CTE) & Central Loose $e$ (CLE) & Plug $e$ (PLE) & Phoenix $e$ (PHE) \\
\hline \hline$E_{T}>8 \mathrm{GeV}$ & $E_{T}>5 \mathrm{GeV}$ & $(5) 8<E_{T}<15 \mathrm{GeV}$ & $>15 \mathrm{GeV}$ \\
$\mathrm{p}_{T}>5 \mathrm{GeV}$ & same & - & - \\
Number Tracks $>0$ & same & N Good Si tracks $>0$ & Phoenix Track \\
$|\eta|<1.0$ & same & $1.2<|\eta|<2.0$ & same \\
HadEm $<0.055+0.00045 \cdot E_{E M}$ & same & HadEm $<0.055$ & same \\
Isolation $<0.1 \cdot E_{T}$ & same & same & same \\
$\chi_{\text {strip }}^{2}<10$ & - & $\chi_{P E M}^{2}<10$ & $\chi_{P E M}^{2}<10$ \\
$E / p<2$ (if $\left.\mathrm{p}_{T}<50\right)$ & - & $E / p<3$ & - \\
$L_{\text {share }}<0.2$ & - & N PES clusters $>0$ & PEM $3 \times 3$ Tower $\neq 0$ \\
$|\Delta z| \leq 3$ & - & PES $5 \times 9>0.65$ & PES $5 \times 9>0.65$ \\
$-3.0<q \cdot|\Delta x|<1.5$ & - & - & - \\
\hline
\end{tabular}

Table 6.7

Electron identification efficiency measured in data and in simulated data (statistical uncertainty only).

\begin{tabular}{|c|c|c|}
\hline Type & Efficiency (data) & Efficiency (MC) \\
\hline \hline CTE & $79.9 \pm 0.2 \%$ & $81.4 \pm 0.1 \%$ \\
CLE & $92.3 \pm 0.1 \%$ & $92.6 \pm 0.1 \%$ \\
PLE & $83.7 \pm 0.3 \%$ & $89.7 \pm 0.1 \%$ \\
PHE & $65.8 \pm 0.4 \%$ & $69.1 \pm 0.1 \%$ \\
\hline
\end{tabular}

\subsection{Jets}

Jets at CDF are reconstructed by a fixed cone algorithm [63] [64]. The cone aperture in the $r-\phi$ plane is calculated as $\Delta R=\sqrt{\Delta \eta^{2}+\Delta \phi^{2}}$, in particular in our analysis we use $\Delta R=0.4$. The jet reconstruction algorithm selects seed towers with energy larger than $0.3 \mathrm{GeV}$ and associates to them candidate towers in the hadronic calorimeter with energy larger than $0.1 \mathrm{GeV}$ located within $\Delta R$. At each step, a new jet center is calculated as the $E_{T}$ weighted centroid. Once the candidate jets are found, possible overlaps between two jets are considered. If the overlap fraction is larger than 0.75 , the two jets are merged together (a maximum of two jets can be merged together); otherwise, two separate jets are constructed such that the 
common towers are assigned to the closest jet. In order to build the jet momentum each electromagnetic and hadronic tower is assigned a massless 4-momentum; the magnitude is then given by the tower energy and the 3-momentum points from the event vertex to the center of the tower. The jet momentum is the sum over the towers. Once the jet is reconstructed, its energy needs to be corrected to take into account several detector effects as documented in [65].

- Eta dependence. This correction guarantees an homogeneous response over the entire angular range of the calorimeter, in particular it corrects for "cracks" located at $\eta=0.0$ (between the East and the West side of CDF), at $\eta=1.1$ (between the Central Calorimeter and the Plug Calorimeter) and at $\eta=1.5$.

- $E_{T}$ dependence. The calorimeter is not linear in the response versus $E_{T}$. The correction transforms the calorimeter jet energy into the corresponding particle jet energy.

- "Minimum bias" dependence. Pile up of multiple p $\bar{p}$ interactions per bunch crossing produces additional energy in the jet cones from the hard interaction. The mean number of interactions per bunch crossing depends on the instantaneous luminosity. Typically the pile up contributes to the energy measured in the $\Delta R$ cones with about $0.3 \mathrm{GeV}$ per interaction.

Once a correction factor $k_{j e t}$ for the jet energy is determined, a few straightforward requirements are applied to the reconstructed jets:

- the transverse energy is required to be larger than $5 \mathrm{GeV}$;

- the pseudo-rapidity $\eta$ measured from the origin of the detector to the calorimeter jet is less than 2.5 ;

- the ratio between the electromagnetic energy and the hadronic energy must be less than 0.9 to distinguish between electron or photon and hadronic jets. 


\subsection{Missing Transverse Energy}

When weakly interacting particles traverse the detector they do not release signal in the tracking system and the energy deposited in the calorimeter is negligible. However, based on the conservation of the transverse momentum such invisible particles are detected by the energy imbalance in the detector, known as missing transverse energy or $E_{\mathrm{T}}$. The $E_{\mathrm{T}}$ is calculated as the negative sum of all calorimeter towers within $\eta<3.6$ and with energy larger than $0.1 \mathrm{GeV}$ :

$$
\mathrm{E}_{\mathrm{T} x}=-\sum_{n=1}^{n=N_{\text {towers }}} E_{x}^{n} ; \quad \mathrm{E}_{\mathrm{T} y}=-\sum_{n=1}^{n=N_{\text {towers }}} E_{y}^{n} ; \quad \mathrm{E}_{\mathrm{T}}=\sqrt{{\mathrm{E}_{\mathrm{T} x}^{2}}^{2}+\mathrm{E}_{\mathrm{T} y}^{2}}
$$

The $E_{\mathrm{T}}$ needs to be corrected for the position of the primary vertex since it is reconstructed assuming the vertex located at in $z=0$. Furthermore muons are MIPs, as explained in Section 6.3, therefore the $\mathbb{E}_{\mathrm{T}}$ must be corrected for the actual muon energy as:

$$
{\mathrm{E}_{\mathrm{T} i}}^{\mu-\text { corr }}=\mathrm{E}_{\mathrm{T} i}^{\text {raw }}-\sum_{\text {muon }} p_{T i}^{\text {muon }}+\sum_{\text {muon }} E_{T i}^{\text {muon }}
$$

where $E_{T i}^{m u o n}=2 \mathrm{GeV}$ is the average energy deposited by muons in the calorimeter and $p_{T i}^{m u o n}$ is the muon $p_{T}$. Instead of subtracting the energy measured in the calorimetric towers, we utilize the average energy deposited. The reason is that we want to reproduce the $\mathbb{E}_{\mathrm{T}}$ correction for misidentified muons where the misidentified muon is a track (Section $7.4[66]$ ).

Besides the muons, the $\mathbb{E}_{\mathrm{T}}$ is also affected by any mis-measurement of the jet energy. This effect is taken into account by additionally modifying the $\mathbb{E}_{\mathrm{T}}$ :

$$
{\mathrm{E}_{\mathrm{T} i}}^{\text {corr }}=\mathrm{E}_{\mathrm{T} i}^{\mu-c o r r}+\sum_{j e t}\left(1-k_{j e t}\right) \times E_{T i}^{j e t},
$$

where $k_{j e t}$ is the jet energy correction factor measured in Section 6.5. 


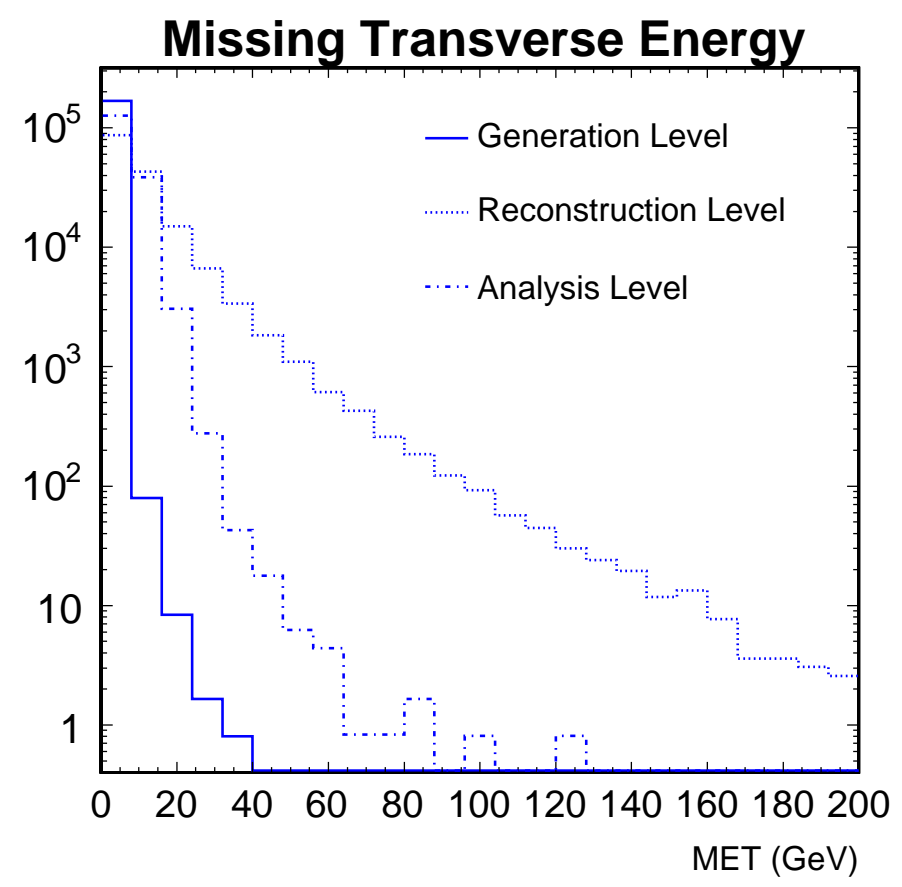

Figure 6.7. Missing transverse energy in Drell-Yan events at generation level, reconstruction level and after the corrections. 


\section{Standard Model backgrounds}

The associated production of chargino and neutralino at the Tevatron could manifest itself in events with three leptons and significant missing transverse energy due to the presence of two LSP's and one neutrino. However this is not a unique signature for SUSY and we do predict a small background. Indeed, there exist several Standard Model processes that yield the same final state and represent a background in our search. The integrated number of SM events could be calculated from the known production cross section and the branching ratio of the particles involved. However, in order to predict the number of events contributing in a particular region of the phase space, a statistical approach has to be taken. A Monte Carlo technique is used to generate an ensemble of events for each given process. After each SM sample is generated, it undergoes the detector simulation which folds in the resolution of each kinematic quantity event by event. It insures that the time dependent detector performance is reproduced at the simulation level to allow data to MC comparison. Typically the size of the MC sample (in terms of luminosity) exceeds the available data sample to reduce the statistical uncertainty on the SM prediction. The generated MC samples are analogous to the data samples. Next, we describe each SM process contributing to the total background, with particular emphasis on the background due to misidentified leptons, which is the only background measured using data.

\subsection{Drell-Yan production}

The Drell-Yan (DY) production is an electroweak process in which a quark and antiquark from the proton and antiproton annihilate to give a lepton pair. The mediator is either the $\gamma$ or the $Z$ boson. At leading order the Drell-Yan results in two leptons per event but an additional lepton might originate from bremsstrahlung. If 


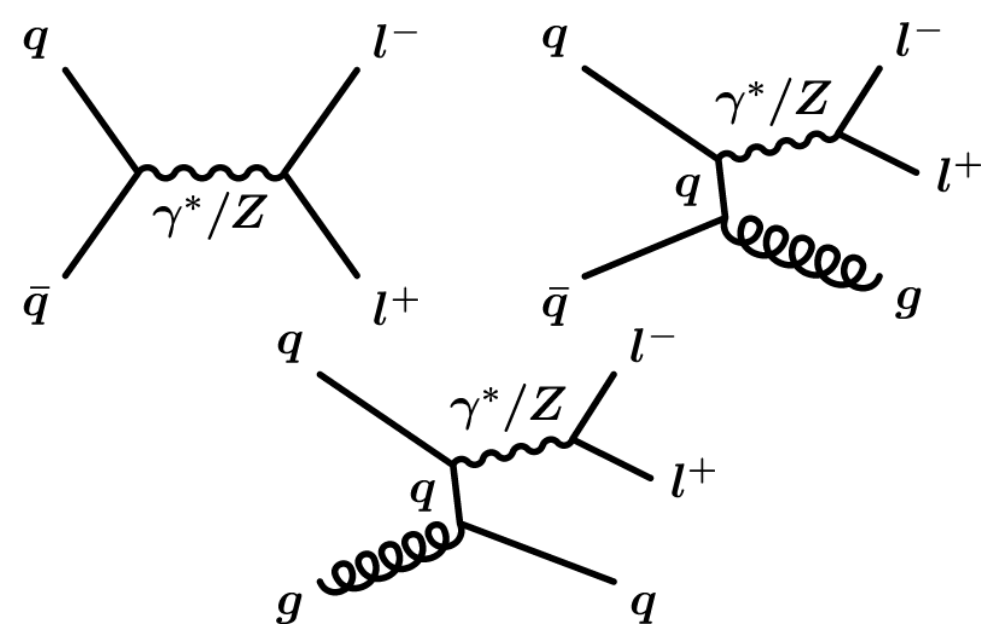

Figure 7.1. LO and NLO Drell-Yan production

one of the daughters radiates a photon and the photon decays into a $e^{+} e^{-}$pair, the Drell-Yan event could contain up to four leptons. A similar final state could be found if the jet from the initial (ISR) or final state radiation (FSR) is misidentified as a lepton. This last contribution will be addressed in Section 7.4. The DY samples are generated with PYHTIA (version 6.216 [67]) at leading order (LO), with different kinematic cuts at generator level depending on the sample. The leading order PYTHIA cross section for the process $Z / \gamma^{*} \rightarrow \ell^{+} \ell^{-}$is $355.2 \pm 3 \mathrm{pb}$ if the dilepton invariant mass is larger than $20 \mathrm{GeV} / c^{2}$; if the cut is placed at $5 \mathrm{GeV} / c^{2}$, the $Z / \gamma^{*} \rightarrow \ell \ell$ cross section increases to $17374.2 \mathrm{pb}$. A so called "k-factor" is applied to take into account NLO effects. In Figure 7.2 the normalization factor is shown as a function of the invariant mass. The Drell-Yan is expected to produce isolated leptons and small $\mathbb{E}_{\mathrm{T}}$ with the exception of the final state with $\tau$ s.

\subsection{Diboson production}

Diboson events are produced by quark and antiquark annihilation. The final state is characterized by two or more leptons, originating from the decay of $W W$, $W \gamma, W Z / \gamma^{*}$ and $Z Z$. The $W W$ and $Z Z$ MC samples are generated using PYHTIA 


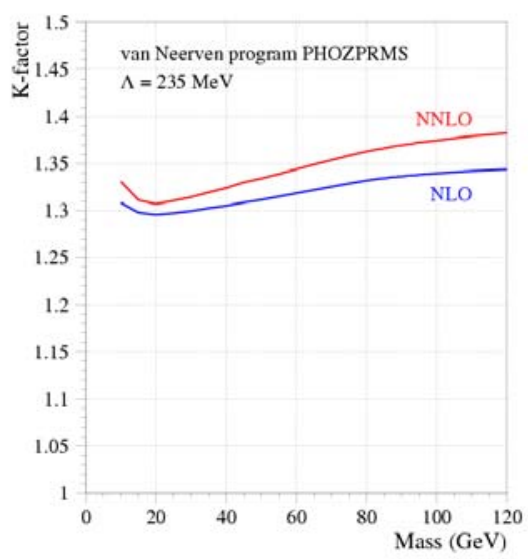

Figure 7.2. k-factor for Drell-Yan production as a function of the invariant mass.

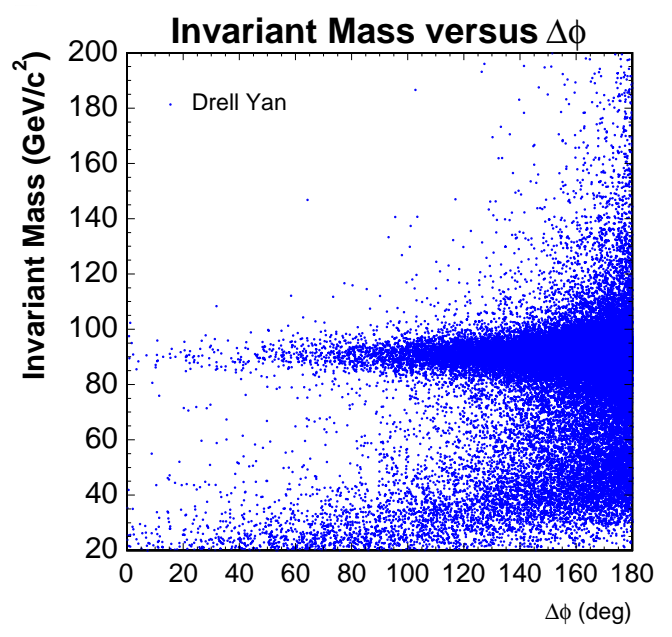

Figure 7.3. Correlation between the invariant mass and the azimuthal distance between muons in Drell-Yan events.

while the event generator MADGRAPH [68] is needed for the $W Z / \gamma^{*}$ process because PYHTIA provides only a Breit Wigner distribution for the Z boson production. We use the theoretical NLO cross section for the $W W$ background, $\sigma_{q \bar{q} \rightarrow W W}=12.4 \pm 0.8$ [69]; the cross sections for $Z Z$ and $W Z / \gamma^{*}$ are the LO cross sections provided by 


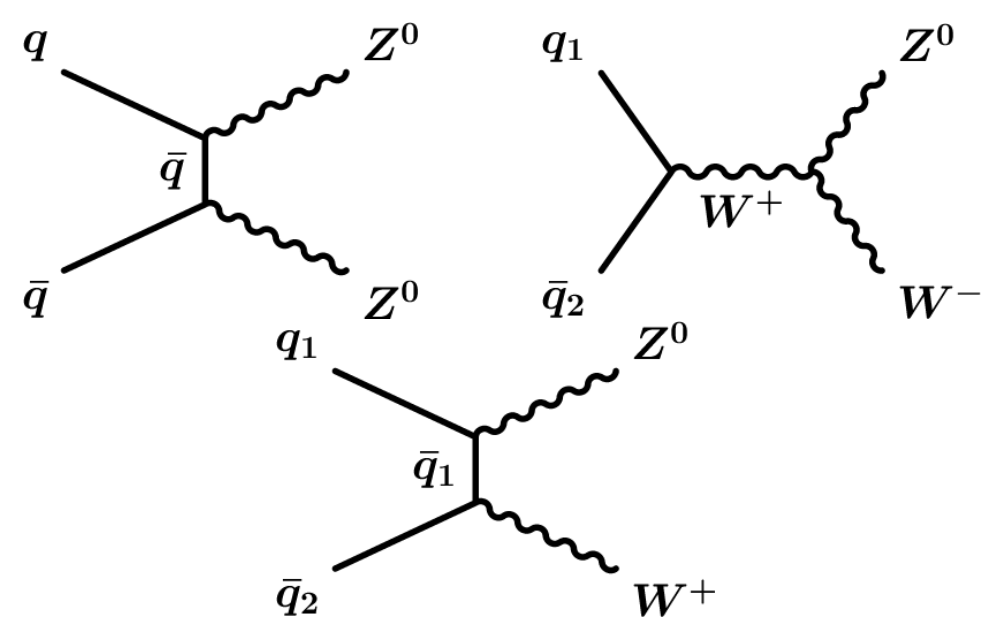

Figure 7.4. LO Diboson production
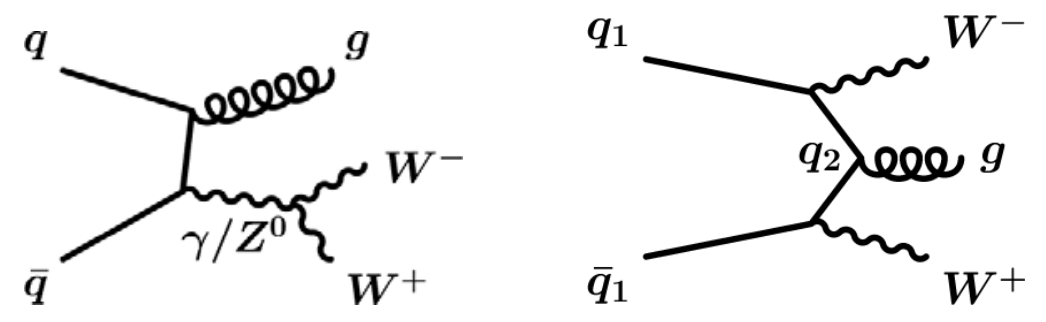

Figure 7.5. NLO Diboson production

the generator, respectively $229 \pm 1 \mathrm{fb}^{1}$ and $208 \pm 1 \mathrm{fb}$. The LO cross sections are corrected to NLO by a k-factor as in the case of Drell-Yan production. When the weak bosons decay, they yield isolated relatively high $p_{T}$ leptons and often missing transverse energy as shown in Figure 7.6 and Figure 7.7. Diboson production is called "irreducible" background since it mimics the SUSY signal as shown in Chapter 8. However a partial suppression can be achieved if the $Z$ is on shell.

\footnotetext{
${ }^{1}$ Note that this is the production cross section after applying generator level requirements. The theoretical prediction for $Z Z$ production at NLO is $1.39 \pm 0.1 \mathrm{pb}$.
} 


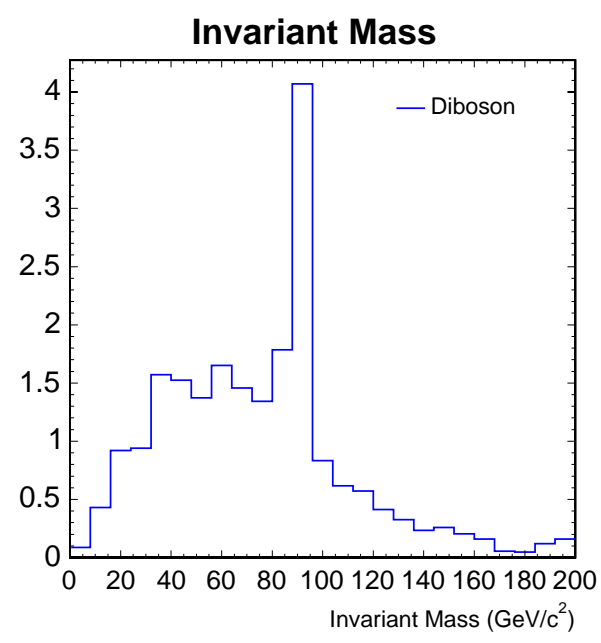

Figure 7.6. Opposite sign muon invariant mass distribution in diboson events (WW, WZ $\left.\gamma^{*}, \mathrm{ZZ}\right)$.

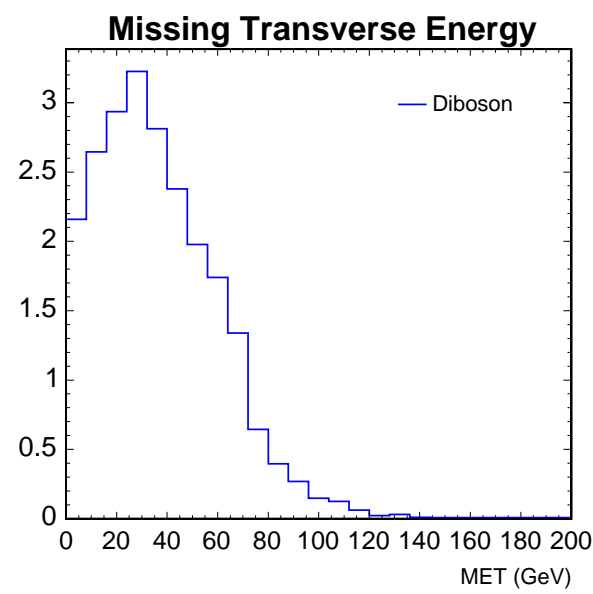

Figure 7.7. Missing transverse energy in diboson events (WW, WZ $\left.\gamma^{*}, \mathrm{ZZ}\right)$.

\subsection{Top pair production}

Top pairs ${ }^{2}$ are produced through quark-antiquark annihilation (15\%) and gluon fusion (85\%). Top quarks are heavy so they do not undergo hadronization and they decay with almost $100 \%$ rate into $W b$. Depending on the boson decay, the final ${ }^{2}$ Light quark production is not a background for our search since the leptons originating in the
semilepton decays of $b$ and $c$ quarks are typically soft $p_{T}$ compared to what we expect from SUSY. 
state could have two leptons, two $b$ jets, and missing transverse energy from the neutrinos. A third lepton can originate from the semileptonic decay of the $b$ quark. A PYTHIA MC sample is generated with the theoretical NLO cross section $\sigma=6.7 \pm 0.3$ $\mathrm{pb}$ [70], in agreement with the measurements at Tevatron [71]. The leptons from
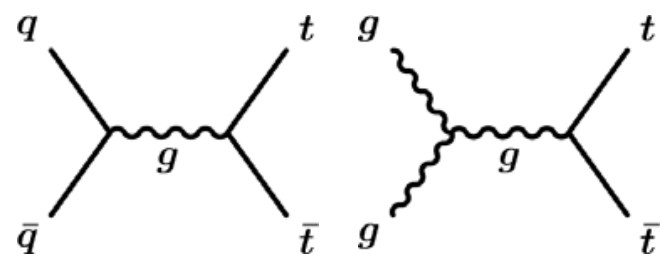
(a) $q \bar{q}$ annihila-
(b) Gluon fusion

tion

Figure 7.8. LO $t \bar{t}$ production production

top decay follow a similar pattern as the one originating from weak boson decays. The missing transverse energy is significant. However, top pair production can be distinguished from SUSY events because it is characterized by large jet activity as shown in Figure7.9.

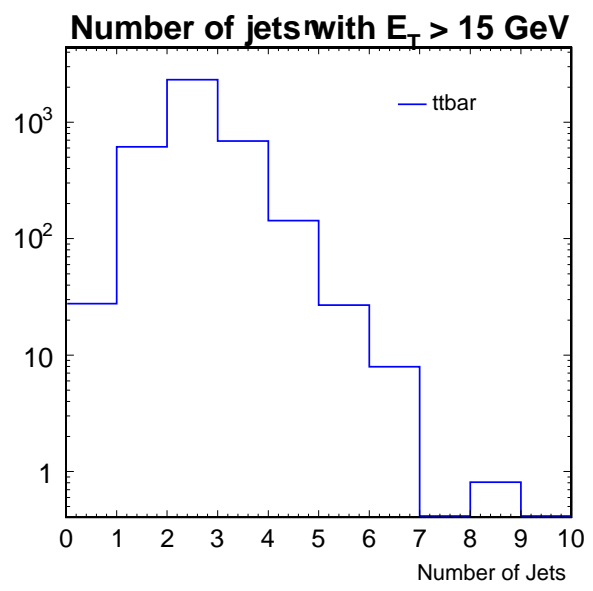

Figure 7.9. Jet multiplicity in $t \bar{t}$ events $\left(\right.$ Jet $\left.\mathrm{E}_{T}>15 \mathrm{GeV}\right)$. 


\subsection{Misidentified leptons}

A jet with large energy deposited in the electromagnetic calorimeter and a track pointing to it can be misidentified as an electron or "fake an electron". If the jet originates from hadron activity such as pions and kaons with a late shower, it can produce a signal in the muon chamber and mimic a muon. Besides jets faking leptons, the data also contain real leptons that however should fail the identification criteria. For instance kaons decaying in flight produce a "'seagull' pattern where the kink corresponds to the decay vertex and can be misidentified as high $p_{T}$ muons. Technically we would need to determine the origin of each lepton and reject the event if the lepton is fake. However, this is not possible since any identified lepton "is" a lepton according to "a priori" criteria. Instead we estimate how many fake leptons do populate the selected events following a statistical approach. We build a sample where one lepton is misidentified. To do so we cannot rely on Monte Carlo simulations since the fakeable objects ${ }^{3}$ typically originate from NLO processes which have large theoretical uncertainty, hadronization of quarks and gluons in minimum bias and underlying event $\left(\mathrm{UE}^{4}\right)$ which are modelled in $\mathrm{MC}$, and from QCD production whose simulation suffers from low statistics. Instead we utilize the data to achieve a more accurate description of the contribution due misidentified leptons; in particular we adopt the search data sample to gain internal consistency. We start by selecting events with two identified leptons ${ }^{5}$. On an event basis, we look for at least one fakeable object, in particular jets could fake electrons and isolated tracks could fake muons. In the analysis we will require the leptons to be separated in space by $\Delta R=\sqrt{\phi^{2}+\eta^{2}}>$ $0.4^{6}$. We apply the same criteria to the fakeable object with respect to the first two

\footnotetext{
${ }^{3}$ We categorize reconstructed objects that could fake leptons into "isolated tracks" and "jets". These will be called "fakeable objects". A fakeable object is a misidentified or fake lepton is the probability to fake the lepton is larger than 0.

${ }^{4}$ Particles from the deep inelastic processes, such as spectator quarks and gluons.

${ }^{5}$ Given the small misidentification probability $\left(\mathcal{P} \sim 10^{-3}\right)$, it is unlikely to find two fake leptons in the same event $\left(\mathcal{P} \sim 10^{-6}\right)$.

${ }^{6}$ If a jet is closer than $0.4 \mathrm{rad}$ to an identified electron different from the two prompt leptons, the electron is considered as an additional fakeable object.
} 
leptons in the event. If there is a third prompt lepton in the event (which is also an isolated track or a jet of course), it is treated as a fakeable object. Such choice leads to a tiny overestimate of the contribution from fake leptons. Once the event is selected we determine the probability for such an event to be identified as a trilepton event. This probability is equal to the probability that the fakeable object is identified as a lepton and becomes the "weight" of the event when the search algorithm is applied to it. In details, if there is only one fakeable object $(F O 1)$, the weight will be the fake rate of the fakeable object (called $f_{1}$ ). The event is then stored in a so called "fake" sample. If the same event contains a second fakeable object (FO2) with fake rate $f_{2}$, the event will be stored again in the fake sample with a weight $f_{2} \times\left(1-f_{1}\right)$. Besides the different weights, the events of course have a different lepton content. The first event contains two prompt leptons and the fakeable object $F O 1$ as a third lepton while the second event will have the fakeable object FO2 as a third lepton. Out of $\mathrm{N}$ events in our search data sample, the total number of events in the fake sample can be written as: $N_{\text {fakeEvents }}=\sum_{k=1}^{N} \sum_{i=1}^{N_{f o}}\left(f_{i k} \times \prod_{j=1}^{i-1}\left(1-f_{j k}\right)\right)$ where $\mathrm{N}$ is the total number of events in the data sample with two real leptons and at least one fakeable object; $N_{f o}$ is the number of fakeable objects in each given event. This background is treated as the Monte Carlo based backgrounds. For instance, the isolated track faking the muon is considered a muon by our search algorithm. However since the track may not be associated to any cluster in the calorimeter, we would need to assign an energy to it. Based on the mean value of the muon energy deposited in the calorimeter, we assume that each fake muon bears a total energy of $2 \mathrm{GeV}$ (see Section 6.3). When a jet fakes an electron, its energy is re-evaluated and a random value of charge is assigned to it.

For our analysis we need to measure the misidentification probability or fake rate. Since the fakeable objects originate from hadrons we utilize three jet samples, each one requiring at least one jet with $E_{T}>20,50,100 \mathrm{GeV}$ respectively [72]. The sample composition is mainly QCD production, and given the small ratio $\frac{\sigma_{E W K}}{\sigma_{Q C D}}$, we 
expect the prompt lepton contribution to be negligible. The jet that fired the trigger is extracted from the fakeable object list to avoid any bias in the measurements. The fake rate is then calculated as the ratio of the identified leptons over the fakeable objects, namely the number of electrons divided by the number of jets or the number of muons over the number of isolated tracks. The fake rate is parametrized in terms of the transverse energy of the jet in case of electrons, or as a function of the transverse momentum of the track in case of muons as:

$$
\mathcal{P}_{\text {jet }}^{i}\left(E_{T}\right)=\frac{N_{I D \text { electron }}^{i}\left(E_{T}\right)}{N_{\text {jet }}^{i}\left(E_{T}\right)}
$$

where $\mathcal{P}_{j e t}^{i}\left(E_{T}\right)$ is the probability that a fakeable jet with $E_{T}$ in the $i^{\text {th }}$ bin fakes an electron. A similar procedure is followed for the isolated track and the muons. The fake rate per fakeable object is finally provided as the average in the three jet data samples. The fake rate calculated in the jet samples is not strictly the same as the fake rate in the analysis dataset since the quark and the gluon fraction differ from sample to sample but the systematic uncertainty assigned to it in Section 9.1 covers the difference. The fake rate as a function of the jet $\mathrm{E}_{T}$ and the track $p_{T}$ is shown

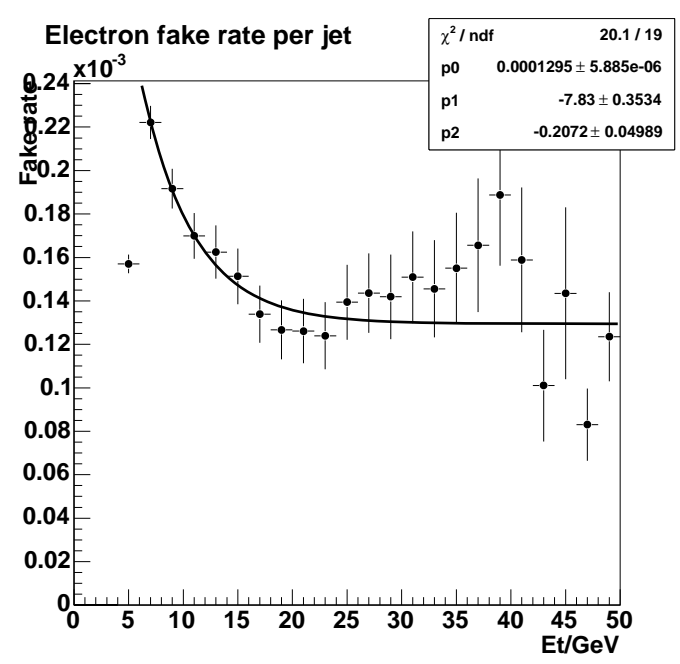

Figure 7.10. Fake rate of Tight Central Electrons

for tight central electron and CMUP muons in Figure 7.10 and Figure 7.11. The fake 


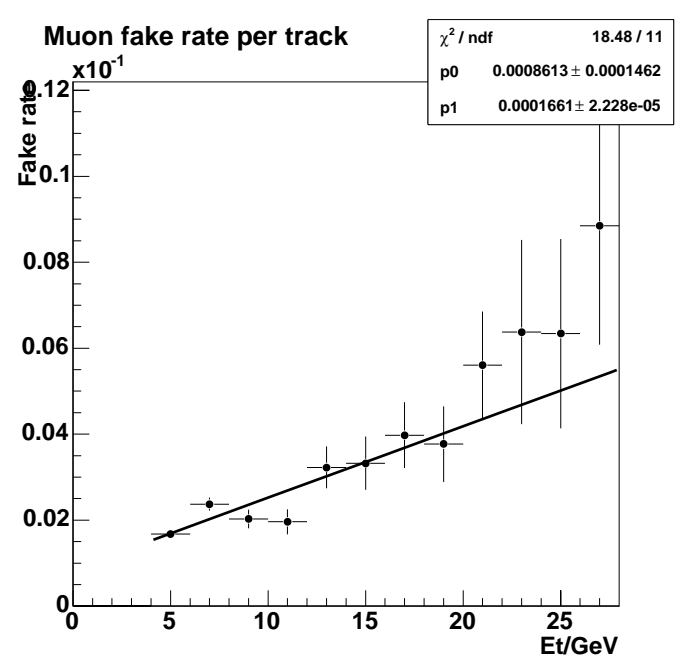

Figure 7.11. Fake rate of CMUP Muons

rate for muons is typically higher than the misidentification probability of electrons since the muon fakeable object already includes some isolation requirement. The fake rate shows an enhancement for $\mathrm{E}_{T} / p_{T} \sim 30 \mathrm{GeV}$ due to the contribution from real electroweak leptons present in the jet samples.

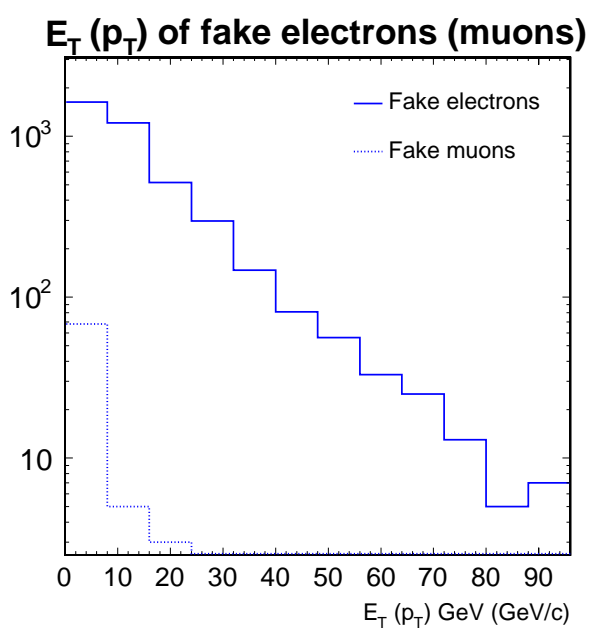

Figure 7.12. Distribution of fakeable objects in the search data sample. 


\subsection{Calculation of the number of expected events}

If we want to calculate the number of background events we expect in a total amount $\mathcal{L}$ of data, we run the search algorithm over the appropriate Monte Carlo sample generated with a luminosity $\mathcal{L}^{\prime}$ and assign each event a weight $p$ :

$$
p=\frac{\mathcal{L}}{\mathcal{L}^{\prime}} \times \epsilon_{t r} \times \prod_{j=0}^{N_{\text {lep }}} \frac{\epsilon_{I D}^{\text {Data }}}{\epsilon_{I D}^{M C}} \frac{\epsilon_{\text {Reco }}^{\text {Data }}}{\epsilon_{\text {Reco }}^{M C}}
$$

The factor $\frac{\mathcal{L}}{\mathcal{L}^{\prime}}$ normalizes the expectation to the proper luminosity while the sum over all (used) leptons in the event $\left(N_{l e p}\right)$ corrects for the different detection efficiency in data and in Monte Carlo (see details in Chapter 6). In particular, the ratio of the efficiency measured in data over the the efficiency measured in MC is called "scale factor". The trigger efficiency $\epsilon_{t r}$ depends on the $p_{T}$ of the muons, as explained in Appendix A, therefore not all muons make it to our data sample. In particular $\epsilon_{t r}$ is equal to the trigger path efficiency, either $\epsilon^{C M U P 18}$ or $\epsilon^{C M X 18}$ if there is only one trigger candidate muon in the event; otherwise it is calculated as

$$
\epsilon_{t r}=\epsilon^{C M U P 18} \times\left(2-\epsilon^{C M U P 18}\right) \quad \text { or } \quad \epsilon_{t r}=\epsilon^{C M X 18} \times\left(2-\epsilon^{C M X 18}\right)
$$

if two muons of same type.

The procedure is repeated for each SM process we believe populate the data and the total number of events is obtained as a sum over all MC contributions. To correctly reproduce the observed data, we add the total number of misidentified events calculated in Section 7.4 to the SM backgrounds determined from MC. 


\section{The search}

We perform a search for chargino-neutralino associated production in $\mathrm{p} \overline{\mathrm{p}}$ data collected by the CDF detector at the Tevatron collider. These supersymmetric particles are expected to decay in final states with three leptons and missing transverse energy. Therefore we explore data collected with high $p_{T}$ single muon triggers as described in Section 5.2.6. The events of interest contain at least one identified high $p_{T}$ muon, as defined in Chapter 6, and two additional first or second generation leptons. The results of our analysis will be combined with the ones of the other searches performed at CDF in Chapter 9.

The trilepton search is reckoned as the golden mode for SUSY discovery at the Tevatron for its small Standard Model backgrounds. Nevertheless, the expected SUSY signal is small as well since the production cross section is less than $1 \mathrm{pb}$. To avoid any bias that could affect the selection criteria we could perform a shape analysis leaving a fitting parameter "blind" or a counting experiment based on a blind "box". In our specific case, the expected small signal for SUSY does not allow for a shape analysis, and we adopt the blind box technique.

\subsection{Blind box analysis technique}

If we define the sensitivity as the ratio of the number of SUSY events $(S)$ over the

uncertainty on the background prediction $(B), \frac{S}{\sqrt{B}}$, the blind counting experiment consists in the following steps:

- the SUSY signal is studied against the SM backgrounds;

- physical quantities discriminating between SUSY and SM are identified; 
- the "analysis cuts" are defined based on the optimization of the sensitivity when possible;

- the SUSY, the SM backgrounds and the data events are then classified into failing/passing the selection criteria;

- the set of data events satisfying all the analysis cuts defines the signal box and is kept blind until the end of the analysis;

- the SM backgrounds and the data are compared in "control regions" where events satisfy a set of selection criteria. In each control region the SUSY signal is negligible by construction and the data are dominated by SM processes;

- if the predicted SM backgrounds describe the data accurately in the control regions, we open the blind box.

If the number of events in the data is significantly larger than the SM prediction, a discovery can be claimed. Otherwise an exclusion limit is set.

\subsection{SUSY Monte Carlo sample}

The analysis is optimized in the mSUGRA benchmark point defined in Section 4.3.2. The appropriate SUSY MC sample is generated with PYTHIA (version 6.216 [67]) and the electroweak scale particle spectrum and BR's are calculated by ISASUSY of ISAJET (version 7.51 [73]). The NLO production cross section, calculated using the algorithm Prospino [19], is $\sigma_{\tilde{\chi}_{1}^{ \pm} \tilde{\chi}_{2}^{0}}=0.64 \mathrm{pb}$ and the leptonic branching ratio is $0.25 \%$.

The result of the search will be interpreted in the mSUGRA and in the "mSUGRA like" scenarios; in particular, in the "mSUGRA like" scenario the masses of the sleptons are degenerate $\left(m_{\tilde{e}}=m_{\tilde{\mu}} \sim m_{\tilde{\tau_{1}}} \sim m_{\tilde{\tau_{2}}}\right)$. We generate two sets of MC samples, the first in the mSUGRA and the second in the "mSUGRA like" scenario", along the ${ }^{1}$ For this we use ISAJET version 7.71. 
benchmark line defined in Section 4.3.2.

Each SUSY event is given a weight for the trigger and detection efficiency according to the procedure described in Section 7.5.

\subsection{Event preselection}

The number of events we start with is of the order of $10^{7}$, reduced by $15 \%$ after we place the requirement of data quality, disregarding the Silicon performance.

The $\mathbb{E}_{\mathrm{T}}$ depends upon the reconstruction of all objects in the event so it is most likely due to mismeasurement if close to a lepton or a jet. In particular, events are removed from the data sample if the $\mathbb{E}_{\mathrm{T}}$ is aligned to the vectorial sum of two identified muon $p_{T}$ s within 2 degrees $^{2}$. This selection is designed to efficiently reject Drell-Yan events

\section{Table 8.1}

Number of events with at least two identified muons before $\left(N_{b}\right)$ and after $\left(N_{a}\right)$ applying the requirement of the azimuthal distance between the missing transverse energy and the dimuon system.

\begin{tabular}{|c|c|c|}
\hline SM background & $N_{b}$ & $N_{a}$ \\
\hline \hline DY & 24,020 & 22,900 \\
Diboson & 37 & 30 \\
$t \bar{t}$ & 15 & 14 \\
DATA & 24,685 & 23,474 \\
\hline
\end{tabular}

(in particular $Z \rightarrow \mu \mu$ ) in the cases where the muons are back-to-back in the $\phi$ space and the highest $p_{T}$ muon is misreconstructed producing mismeasured $E_{\mathrm{T}}$ along its direction. We note that $Z \rightarrow \tau \tau$ is reduced by almost $10 \%$ compared to a decrease of $5 \%$ in the total SM background. The reason is that $\tau$ pairs from Drell-Yan production can decay leptonically giving rise to real $\mathbb{E}_{\mathrm{T}}$ due to neutrinos. The $\tau \mathrm{S}$ are typically boosted and their decay product collimated. As a result, the $\mathbb{E}_{\mathrm{T}}$ can be along the

\footnotetext{
${ }^{2}$ We believe 2 degrees is the most reasonable choice since the calorimeter cracks are 2 degree wide.
} 
direction of the dimuon system. The impact of this selection is instead negligible for top pair and diboson production as shown in Table 8.1.

If a jet is only partially reconstructed because if it falls in a crack, the amount of energy not deposited in the calorimeter is interpreted in terms of missing transverse energy. This effect is taken into account when the $\mathrm{E}_{\mathrm{T}}$ is recalculated after correcting the jet energy as described in Section 6.5. However the missing transverse energy could still be mismeasured and tends to be aligned with the mismeasured jet as shown in Figure 8.1. Since the cone size used for reconstructing jets is $\Delta R=0.4$, we reject events if $\Delta \phi_{\min }\left(\right.$ jet, $\left.\mathbb{E}_{\mathrm{T}}\right)<20$ degrees thus reducing the number of dimuon events from $23,474(22944)$ to 21,893 (21766) in data (SM prediction). The contribution from DY production is partially suppressed because of possible mismeasurement of ISR/FSR jets. In case of $t \bar{t}$ production, the cut is not efficient since the neutrinos in the event modify the direction of the mismeasured $\mathbb{E}_{\mathrm{T}}$, if any. The efficiency of the selection in dimuon events can be found in Table 8.2.

A $4 \%$ contamination from cosmic ray muons survived the timing selection cut de-

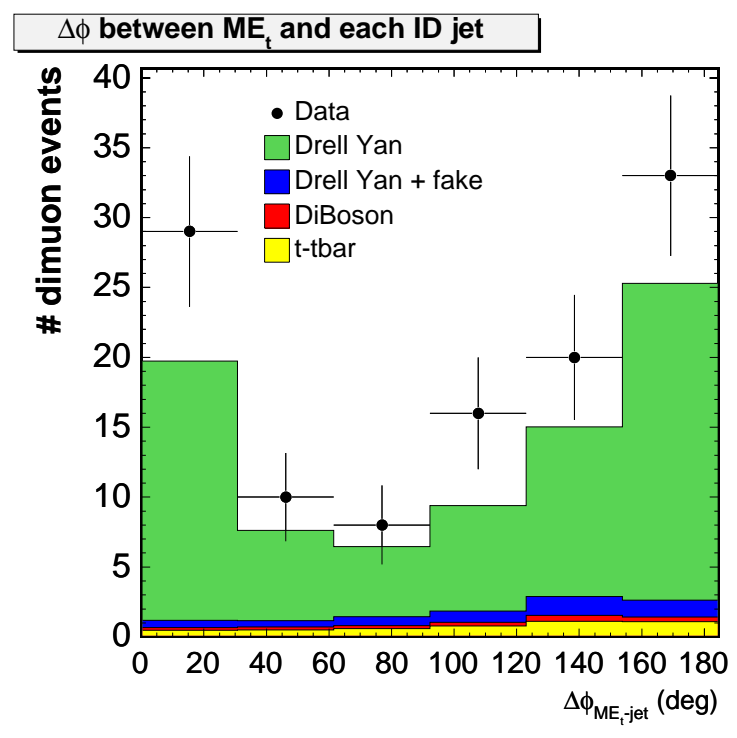

Figure 8.1. Azimuthal distance between the missing transverse energy and each identified jet in events with two muons. 
Table 8.2

Number of events with at least two muons before $\left(N_{b}\right)$ and after $\left(N_{a}\right)$ applying the requirement of the azimuthal distance between the missing transverse energy and the jets with $E_{T}>5 \mathrm{GeV}$.

\begin{tabular}{|c|c|c|}
\hline & $N_{b}$ & $N_{a}$ \\
\hline \hline DY & 22,900 & 21,724 \\
Diboson & 30 & 28 \\
$t \bar{t}$ & 14 & 14 \\
DATA & 23,474 & 21,893 \\
\hline
\end{tabular}

scribed in Section 6.3 and is suppressed by rejecting events where two reconstructed muons are back-to-back in space ${ }^{3}$.

\subsection{Topological cuts}

Charginos and neutralinos are expected to be heavier than 100 and $50 \mathrm{GeV} / c^{2}$ respectively and to decay promptly into leptons and $\tilde{\chi}_{1}^{0} \mathrm{~s}$. In addition to cutting on the impact parameter, all leptons in the event must originate from the interaction point. This is insured by requiring the leptons to be within $4 \mathrm{~cm}$ from the primary vertex along the beam direction and by requiring $\Delta Z_{\ell_{i} \ell_{j}}<4 \mathrm{~cm}$, where $\ell_{i}$ and $\ell_{j}$ denote any two leptons. The leptons should also be separated in $\eta-\phi$ space as $\Delta R_{\ell_{i}, \ell_{j}}=\sqrt{\Delta \phi_{\ell_{i}, \ell_{j}}^{2}+\Delta \eta_{\ell_{i}, \ell_{j}}^{2}}>0.4$.

Since the data sample under investigation is collected via high $p_{T}$ muon triggers, each leading lepton has to be a muon with $p_{T}>20 \mathrm{GeV} / \mathrm{c}$ to avoid any trigger "turnon" effect. Depending on the next-to-leading lepton (NLL) and the third lepton, the analysis is split into channels that will be combined into a final result. The channels will be called $\mu-\mu$ (the NLL is a muon), $\mu-\mathrm{CTE}$ (the NLL is a central tight electron)

\footnotetext{
$\left.\overline{{ }^{3} \text { We define the acollinearity as } \alpha=\operatorname{acos}(} \frac{\overline{p_{1}} \cdot \overline{p_{2}}}{p_{1} p_{2}}\right)$, where $\overline{p_{i}}$ is the muon 3D-vector. If the reconstructed muon pair is a single cosmic ray the muons are reconstructed as back-to-back in space. We require $\alpha<3.1$.
} 
and $\mu-\mathrm{PLE} / \mathrm{PHE}$ (the NLL is a plug or phoenix electron), as defined in Chapter 6 . The overview of the lepton combinations is given in Table $8.3^{4}$.

Finally, the numbers of events with three leptons in data, SM backgrounds and

Table 8.3

Definition of the analysis channels based on the lepton selection.

\begin{tabular}{|c|c|}
\hline Next-to-leading Lepton & Third lepton \\
\hline \hline CMUP/CMX $\left(p_{T}>5 \mathrm{GeV} / c\right)$ or CMIO & same or CLE \\
\hline $\mathrm{CTE}$ & $\begin{array}{c}\text { same or CMUP } / \mathrm{CMX}\left(p_{T}>5 \mathrm{GeV} / c\right) \\
\text { or CMIO or CLE or PLE } / \mathrm{PHE}\end{array}$ \\
\hline $\mathrm{PLE} / \mathrm{PHE}$ & same or CMUP/CMX $\left(p_{T}>5 \mathrm{GeV} / c\right)$ \\
& or CMIO or CLE \\
\hline
\end{tabular}

expected SUSY signal are presented in Table 8.4. We quote the statistical uncertainty for the MC based backgrounds and the systematic uncertainty for the contribution from misidentified leptons as described in Section 9.1. The statistical significance is too low to draw any conclusion about the observation of New Physics. Therefore we identify kinematic and topological quantities to discriminate between SM and SUSY processes to increase the sensitivity of our analysis. The analysis cuts are designed to highly reject the SM backgrounds while preserving the SUSY signal.

\section{Invariant Mass requirement}

The most intuitive and powerful observable to distinguish between the Standard Model backgrounds and the SUSY signal is the invariant mass of same flavor opposite sign leptons shown in Figure 8.2(a). The SM background is dominated by Drell-Yan events where the third lepton originates from $\gamma_{\mathrm{s}}$ converting in the material or from hadrons misidentified as leptons. The on-shell $Z$ contribution can be suppressed by ${ }^{4}$ If the third lepton is a muon the fractional isolation is replaced by the loose isolation as in Table 6.2 . 
Table 8.4

Number of events with at least three leptons.

\begin{tabular}{|c|c|c|}
\hline & $\mu-\mu$ & $\mu$-e channels \\
\hline \hline SUSY & $2.0 \pm 0.8$ & $1.2 \pm 0.3$ \\
SM & $18.1 \pm 2.6$ & $8.0 \pm 0.4$ \\
DATA & 14 & 5 \\
\hline
\end{tabular}

selecting events with the invariant mass outside the mass window from 76 to 106 $\mathrm{GeV} / c^{2}$. Similarly the low mass resonances such as $J / \Psi$ and $\Upsilon$ are removed by requiring a dilepton invariant mass larger than $15 \mathrm{GeV} / c^{2}$. In contrast, the diboson productions $\mathrm{WZ} / \gamma^{*}$ and $\mathrm{ZZ}$ are suppressed only when the $Z$ is on shell. The results of the selection in the $\mu-\mu$ channel are presented in Table 8.5.

Table 8.5

Number of events with at least three leptons after the invariant mass requirement in the $\mu-\mu$ channel.

\begin{tabular}{|c|c|}
\hline & $\mu-\mu$ \\
\hline \hline SUSY & $1.78 \pm 0.07$ \\
DY & $4.55 \pm 0.53$ \\
Diboson & $0.29 \pm 0.02$ \\
$t \bar{t}$ & $0.02 \pm 0.01$ \\
Fake events & $0.68 \pm 0.34$ \\
DATA & 6 \\
\hline
\end{tabular}




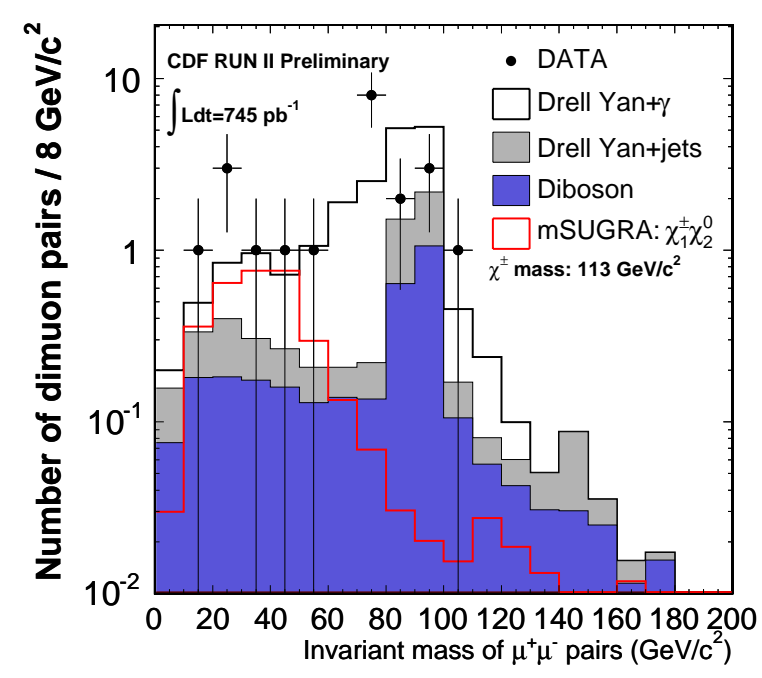

(a) $\mu-\mu$ channel

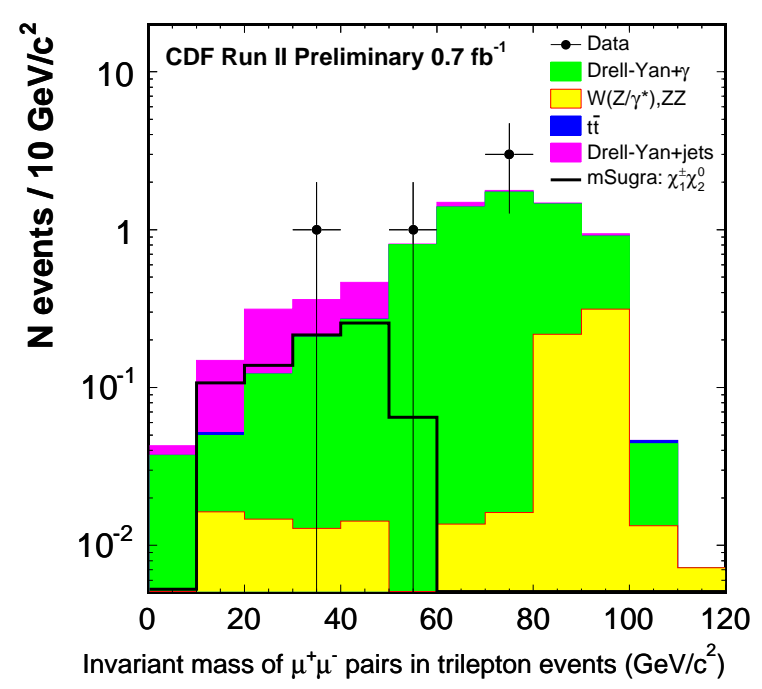

(b) $\mu-e$ channels

Figure 8.2. Invariant mass distribution of opposite sign muons in vents with at least three leptons. 


\section{Jet requirement}

We are interested in SUSY events where the supersymmetric particles decay into leptons. As a consequence we do not expect jet activity except from initial radiation. Besides, there might be jet contributions from underlying event and min bias, in particular in the high instantaneous luminosity regime. As a consequence, the selection criteria based on the number of jets in the event must be a trade-off between wanting to veto jets and not removing too much signal. We select identified jets with $E_{T}>20 \mathrm{GeV}$ and reject the event if it contains more than 1 jet. This criteria helps reducing the remaining heavy flavor background and partially suppresses the fake contamination. However, we do not expect a large impact on EWK processes. The jet multiplicity in events passing the invariant mass cut is shown in Figure 8.3(a) and Table 8.7 presents the impact of the jet cut in the $\mu-\mu$ channel.

Table 8.6

Number of events with at least three leptons after the invariant mass and jet multiplicity requirements in the $\mu-\mu$ channel

\begin{tabular}{|c|c|}
\hline & $\mu-\mu$ \\
\hline \hline SUSY & $1.75 \pm 0.07$ \\
DY & $4.4 \pm 0.5$ \\
Diboson & $0.29 \pm 0.02$ \\
$t \bar{t}$ & $0.01 \pm 0.01$ \\
Fake events & $0.66 \pm 0.33$ \\
DATA & 6 \\
\hline
\end{tabular}

\section{MET requirement}

The Drell-Yan production is still the dominant contribution accounting for up to $80 \%$ of the total background in the $\mu-\mu$ channel. SUSY events are characterized 


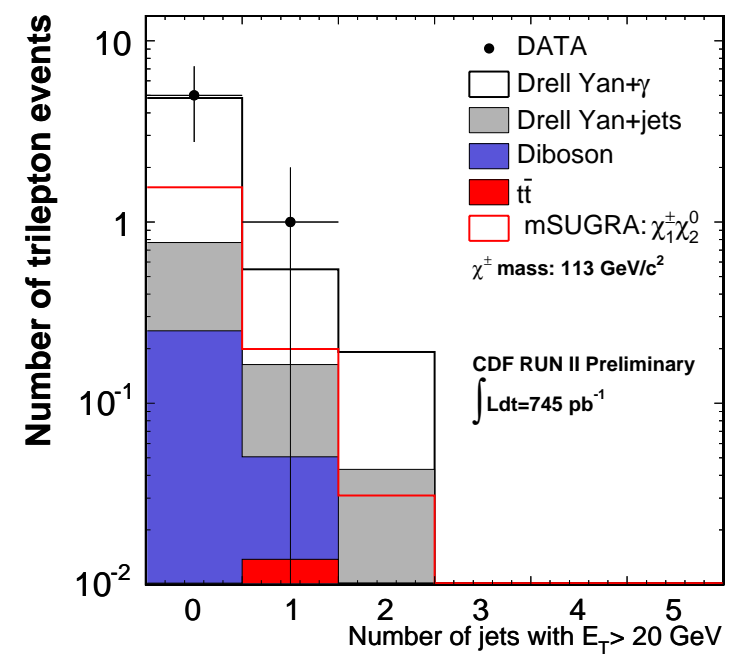

(a) $\mu-\mu$ channel

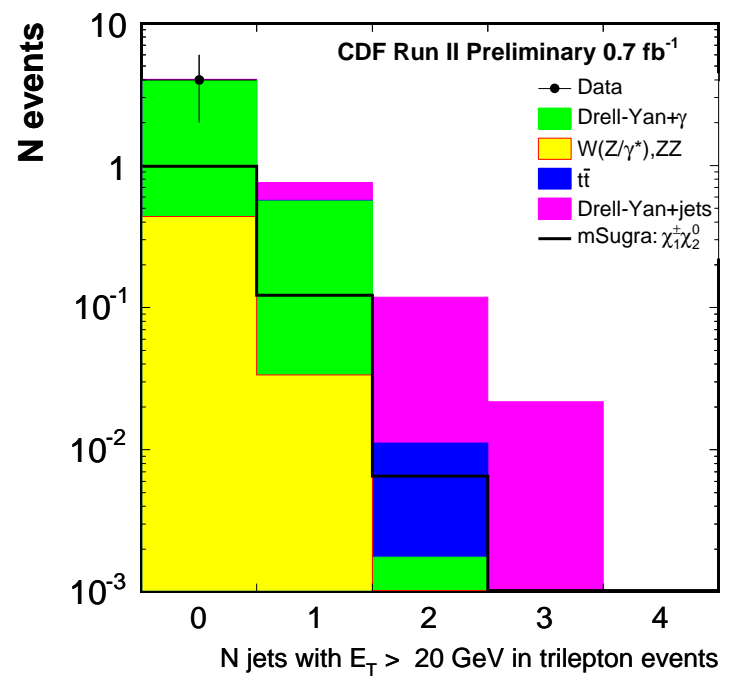

(b) $\mu-e$ channels

Figure 8.3. Number of jets with $E_{T}>20 \mathrm{GeV}$ in events with at least three leptons after the invariant mass requirement. 
by significant missing transverse energy from the LSP's and the neutrinos. This pattern dramatically differs from Drell-Yan production, where only $Z \rightarrow \tau \tau$ exhibit real $\mathbb{E}_{\mathrm{T}}(Z \rightarrow \tau \tau$ is scaled down by the leptonic branching ratio of the $\tau$ with respect to $Z \rightarrow \mu \mu)$. The $\mathbb{E}_{\mathrm{T}}$ cut is optimized based on the sensitivity $\frac{S}{\sqrt{B}}$ as shown in Figure 8.4 and a threshold value of $15 \mathrm{GeV}$ is set. It is crucial to notice that the

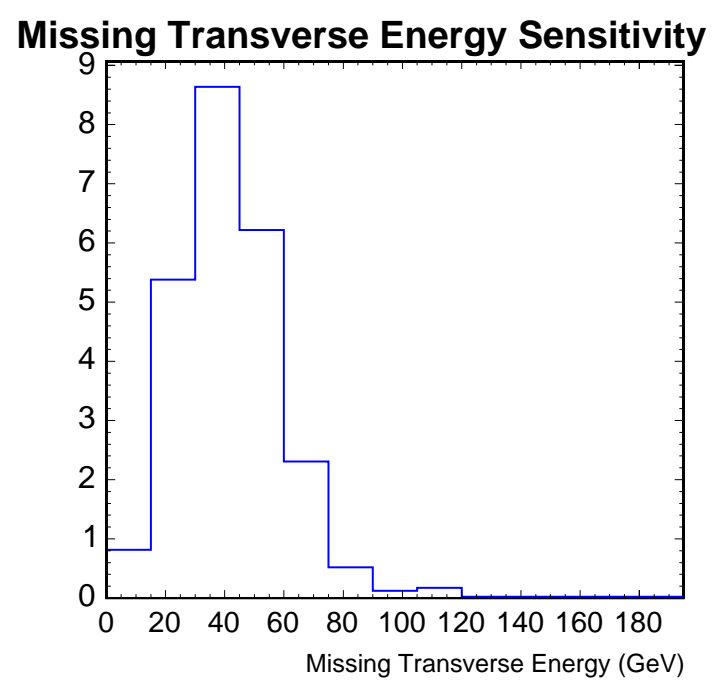

Figure 8.4. Sensitivity as a function of the missing transverse energy threshold in the $\mu-\mu$ channel.

three sequential cuts, the invariant mass of same flavor opposite sign leptons, the jet multiplicity and the missing transverse energy define our "signal box". Therefore the comparison to data in Figure 8.5(a) and Figure 8.5(b) is not extended to the subset of events simultaneously satisfying all of these requirements.

\subsection{Signal Box}

The sensitivity $\frac{S}{\sqrt{B}}$ of the analysis improves from 0.5 to 2 for the $\mu-\mu$ channel and from 0.4 to 1.2 in the $\mu-\mathrm{e}$ channels after all selection criteria are applied. The numbers of expected events in the signal box are shown in Table 8.8 for the three channels considered independently. We note that the analysis in the $\mu-\mathrm{PLE} / \mathrm{PHE}$ 


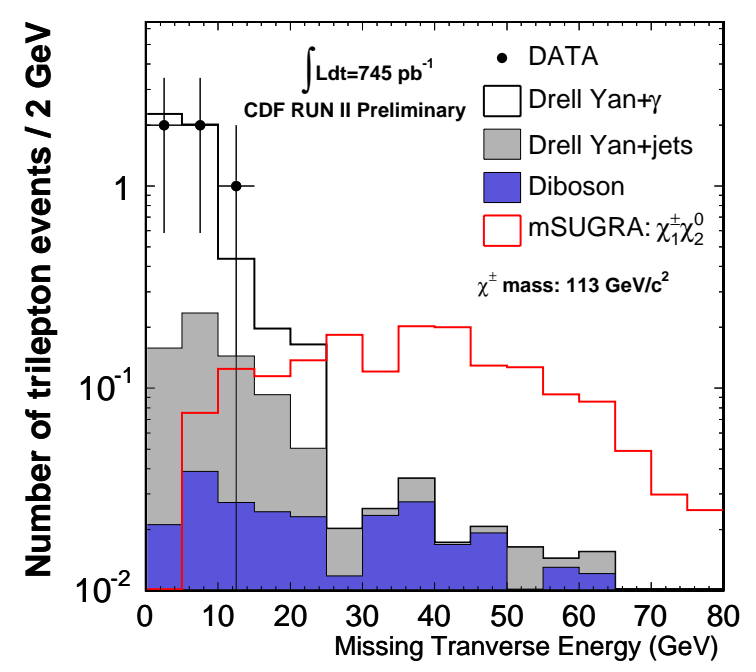

(a) $\mu-\mu$ channel

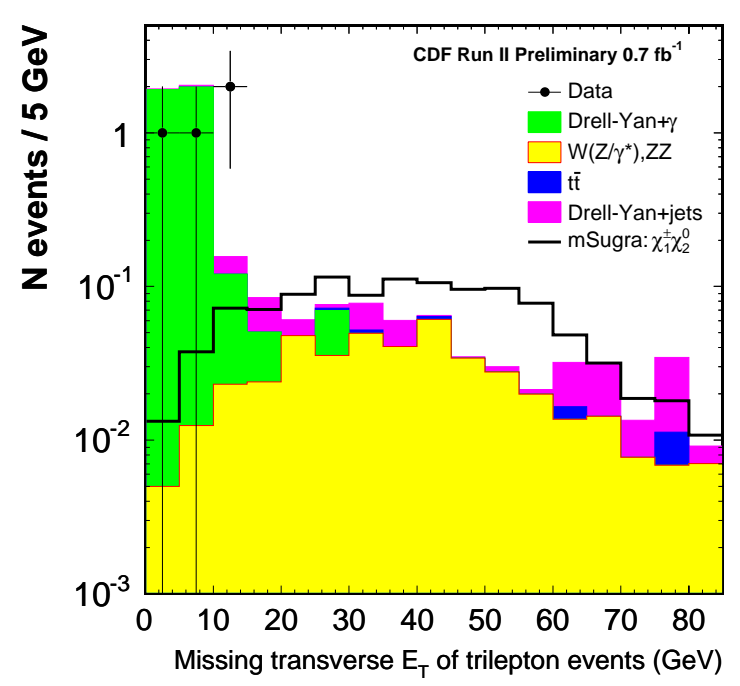

(b) $\mu-e$ channels

Figure 8.5. Missing transverse energy in events with at least three leptons after the invariant mass and jet multiplicity requirements. The data are not plotted in the region where the missing transverse energy is larger than $15 \mathrm{GeV}$. 
Table 8.7

Number of events with at least three leptons after the invariant mass, the jet multiplicity and the missing transverse energy requirements in the $\mu-\mu$ channel.

\begin{tabular}{|c|c|}
\hline & $\mu-\mu$ \\
\hline \hline SUSY & $1.55 \pm 0.07$ \\
DY & $0.22 \pm 0.11$ \\
Diboson & $0.20 \pm 0.02$ \\
$t \bar{t}$ & $0.01 \pm 0.01$ \\
Fake events & $0.2 \pm 0.1$ \\
DATA & $?$ \\
\hline
\end{tabular}

channel is performed on $\mathcal{L}=680 \pm 41 \mathrm{pb}^{-1}$ of data because of different data quality requirements.

Table 8.8

Number of expected events in the $\mu-\mu$ and the $\mu-$ e signal boxes.

\begin{tabular}{|c|c|c|c|}
\hline Process & $\mu-\mu$ Channel & $\mu+\mathrm{CTE}$ & $\mu+\mathrm{PLE} / \mathrm{PHE}$ \\
\hline \hline $\mathrm{DY}+\gamma$ & $0.22 \pm 0.11$ & $0.10 \pm 0.04$ & $0.04 \pm 0.04$ \\
$\mathrm{WW}-\mathrm{WZ} \gamma^{*}-\mathrm{W} \gamma$ & $0.20 \pm 0.02$ & $0.19 \pm 0.02$ & $0.25 \pm 0.03$ \\
$\mathrm{t} \bar{t}$ & $0.014 \pm 0.006$ & $0.009 \pm 0.005$ & $0.007 \pm 0.004$ \\
Fake events & $0.20 \pm 0.10$ & $0.11 \pm 0.55$ & $0.06 \pm 0.03$ \\
\hline Total background & $0.64 \pm 0.11$ & $0.42 \pm 0.05$ & $0.36 \pm 0.05$ \\
Expected SUSY signal & $1.6 \pm 0.1$ & $0.83 \pm 0.06$ & $0.20 \pm 0.02$ \\
\hline
\end{tabular}




\subsection{Understanding the SM backgrounds}

The foundation of a blind analysis is the concept of "control regions", which are defined by the analysis cuts. We remind the reader that the "signal box" for the search of chargino and neutralino is the set of events with:

- at least one high $p_{T}$ muon and two additional leptons;

- invariant mass of opposite sign same flavor leptons must be in the window from 15 to $76 \mathrm{GeV} / c^{2}$ or larger than $106 \mathrm{GeV} / c^{2}$;

- less than 2 identified jets with transverse energy larger than $20 \mathrm{GeV}$;

- missing transverse energy larger than $15 \mathrm{GeV}$.

We can define up to 17 correlated control regions per channel by requiring at least one of the above analysis criteria to fail. Since we have very few events with three leptons, we achieve a statistically significant comparison between the observation and the SM predictions in the control regions with two leptons. The control regions with at least three leptons insure the understanding of low statistics "signal" like events. A sketch of the control regions is shown in Figure 8.6.

\subsubsection{Control regions with two muons}

In the particular case of events with two muons, a significant test of the agreement between $\mathrm{MC}$ and data consists in calculating the cross section of the process $q \bar{q} \rightarrow$ $Z \rightarrow \mu \mu$, as

$$
\sigma_{q \bar{q} \rightarrow Z \rightarrow \mu \mu}=\frac{N^{D A T A}}{\mathcal{L} \times \mathcal{A} \times \varepsilon}
$$

where $N^{D A T A}$ is the number of $\mathrm{Z} \rightarrow \mu \mu$ candidate events in data with invariant mass in the interval between 66 and $116 \mathrm{GeV} / c^{2}$. For this study we can assume that 


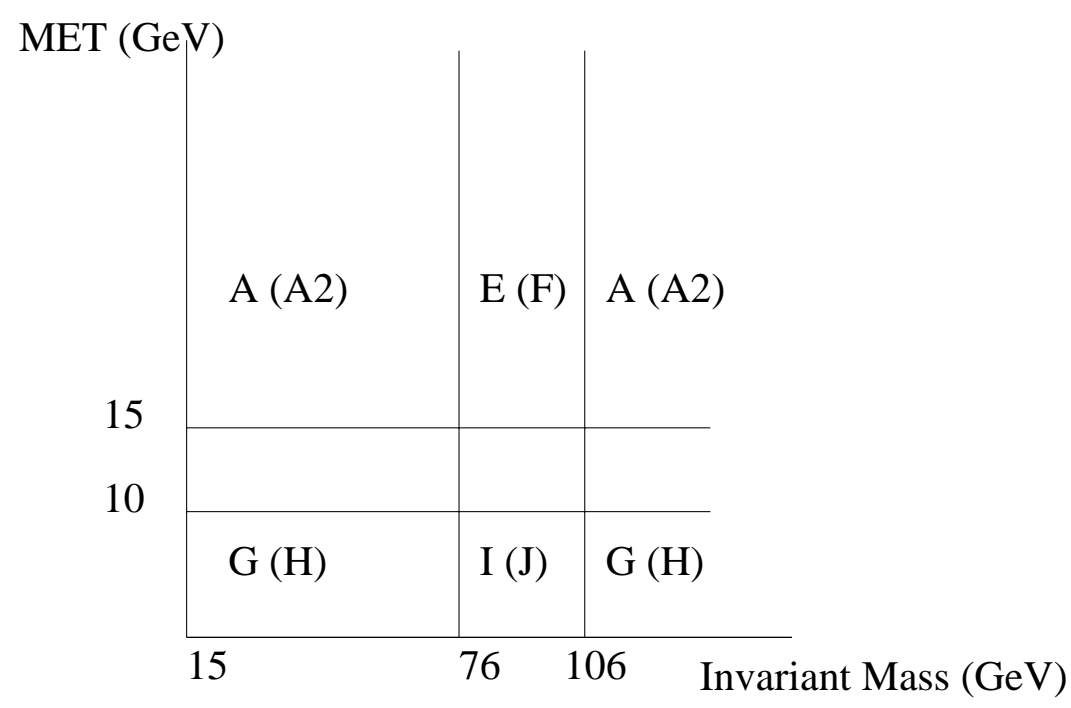

Figure 8.6. Sketch of the control regions. Control regions A, E, G and $\mathrm{I}(\mathrm{A} 2, \mathrm{~F}, \mathrm{H}$ and $\mathrm{J})$ are such that the jet cut is satisfied (fails). Each control region is examined with two and three leptons. Control region A is examined only with two leptons since it becomes the signal box if three leptons are required.

all backgrounds are negligible compared to the $\mathrm{Z} \rightarrow \mu \mu$ process. $\mathcal{L}$ is the integrated luminosity of the data sample and $\mathcal{A}=\frac{N_{\text {reconstructed }}^{M C}}{N_{\text {generated }}^{\text {Me }}}$ is the acceptance calculated from the Drell-Yan MC sample selecting events with the generator level invariant mass in the interval between 66 and $116 \mathrm{GeV} / c^{2}$ and within $\left|z_{0}\right| \leq 60 \mathrm{~cm}$. In the numerator we are requiring the two muons to have a reconstructed mass in the range from 66 and $116 \mathrm{GeV} / c^{2}$. Both muons must be in the fiducial regions of either $\mathrm{CMU}$ and CMP or CMX and have $\mathrm{p}_{T} \geq 20 \mathrm{GeV} / \mathrm{c}$. The efficiencies needed for calculating the cross section are the trigger, reconstruction and identification efficiencies measured in data. In the case of same category muons, the total efficiency $\epsilon$ can be expressed as

$$
\epsilon=\epsilon_{z 0} \epsilon_{\text {Trigger }}\left(2-\epsilon_{\text {Trigger }}\right)\left(\epsilon_{I D} \epsilon_{\text {Reco }}\right)^{2}
$$

where $\epsilon_{z 0}$ is the vertex reconstruction efficiency quoted in Section 6.2, $\epsilon_{I D, i}\left(\epsilon_{R E C O, i}\right)$ is the identification (reconstruction) efficiency for the $i^{\text {th }}$ type muon and $\epsilon_{\text {Trigger }, j}$ the 
trigger efficiency for the $j^{\text {th }}$ type trigger ${ }^{5}$. The results in Table 8.9, broken up in different muon categories, are in agreement with the SM predictions [74] and ensure a good understanding of the luminosity measurement, trigger efficiency and high $p_{T}$ muon detection efficiencies that we evaluated in Chapter 6. Figure 8.7 shows the comparison between the observation and the prediction in terms of muon invariant mass.

Table 8.9

Cross sections of the process $Z \rightarrow \mu \mu$ (statistical uncertainty and systematic uncertainty due to luminosity measurement quoted in Section $9.1)$.

\begin{tabular}{|c|c|}
\hline Muon Type & Cross section (pb) \\
\hline \hline CMUP-CMUP & $245 \pm 6 \pm 15$ \\
CMX-CMX & $236 \pm 12 \pm 14$ \\
CMUP-CMX & $243 \pm 6 \pm 15$ \\
\hline
\end{tabular}

We then study the events in the $\mathrm{Z}$ mass window from 76 to $106 \mathrm{GeV} / c^{2}$ as a function of the missing transverse energy and jet multiplicity presented in Figure 8.8. We expect the low $\mathbb{E}_{\mathrm{T}}$ control region I to be dominated by Drell-Yan production while diboson events contribute to the control region E at large missing transverse energy. The latter is crucial in understanding the resolution of this observable since misreconstructed events typically cluster in the tail of the $\mathbb{E}_{\mathrm{T}}$ distribution. The agreement between the observation and the prediction shown in Table 8.10 proves that the SM backgrounds are properly taken into account and the $\mathbb{E}_{\mathrm{T}}$ correction for muons does not introduce any mismeasurement. The correction for the jet energy can be tested in region $\mathrm{F}$ where we expect $Z+$ jets and $t \bar{t}$ events. In order to reproduce the data,

${ }^{5}$ If the event contains two muons of different category, the total efficiency is expressed as:

$\epsilon=\epsilon_{z 0} \epsilon_{I D, C M U P} \epsilon_{\text {Reco }, C M U P} \epsilon_{I D, C M X} \epsilon_{\text {Reco }, C M X}\left(\epsilon_{\text {Trigger } C M U P}+\epsilon_{\text {Trigger }, C M X}-\epsilon_{\text {Trigger } C M U P}\right.$. $\left.\epsilon_{\text {Trigger }, C M X}\right)$. 


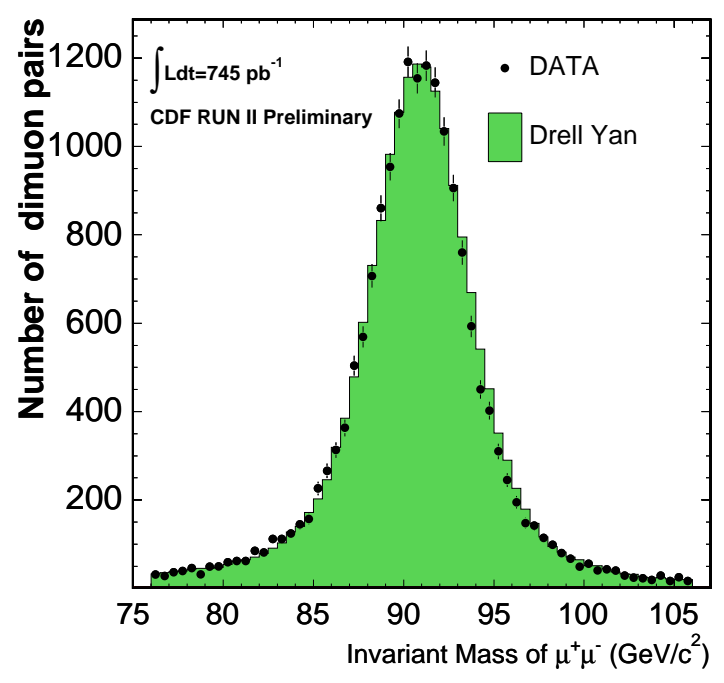

Figure 8.7. Invariant mass of opposite sign muons in dimuon events in the region from 76 to $106 \mathrm{GeV} / c^{2}$.

the SM should also include jets from ISR/FSR along with the contributions from underlying event and minimum bias events. In region $\mathrm{F}$ we predict and observe 31 events.

Once the so called "CDF standard candles", on-shell $Z \mathrm{~s}$, are well reproduced by our MC, we study events satisfying the invariant mass analysis criteria. Figure 8.9(a) compares the predicted missing transverse energy to the one measured in data. The agreement confirms our understanding of the scaling applied for medium $p_{T}$ muons to account for the difference between the detection efficiency in data and in MC. However it also discloses a small discrepancy in the tail of the $\mathbb{E}_{\mathrm{T}}$ distribution likely due to a large uncertainty on the jet multiplicity, presented in Figure 8.9(b); it mainly affect control regions $\mathrm{H}$ and A2. The observed discrepancy could be due to several effects. First, the contribution from misidentified leptons might be underestimated in these control regions. In dimuon events, it is measured by counting the number of events with two same sign isolated muons and correcting this estimate to account for 


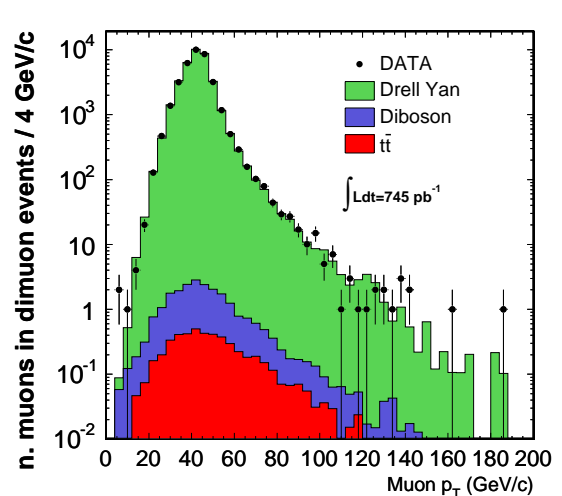

(a) Muon $p_{T}$.

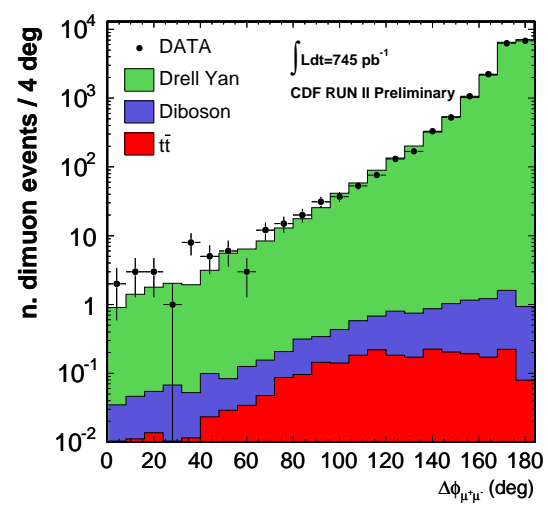

(c) Angular distance between the muons.

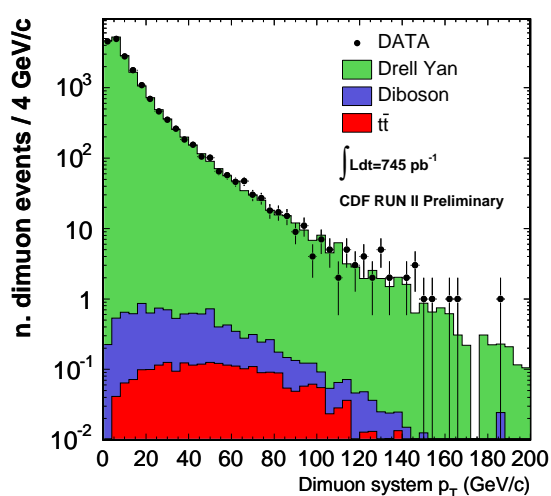

(b) Z $p_{T}$.

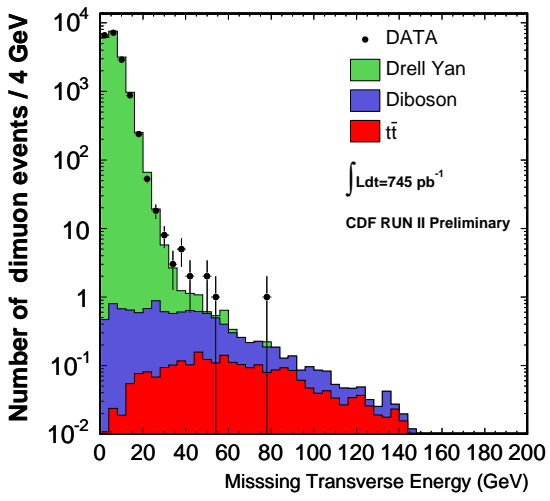

(d) Missing transverse energy.



(e) Jet multiplicity.

Figure 8.8. Kinematical distributions for dimuon events with invariant mass in the range from 76 to $106 \mathrm{GeV} / c^{2}$ 


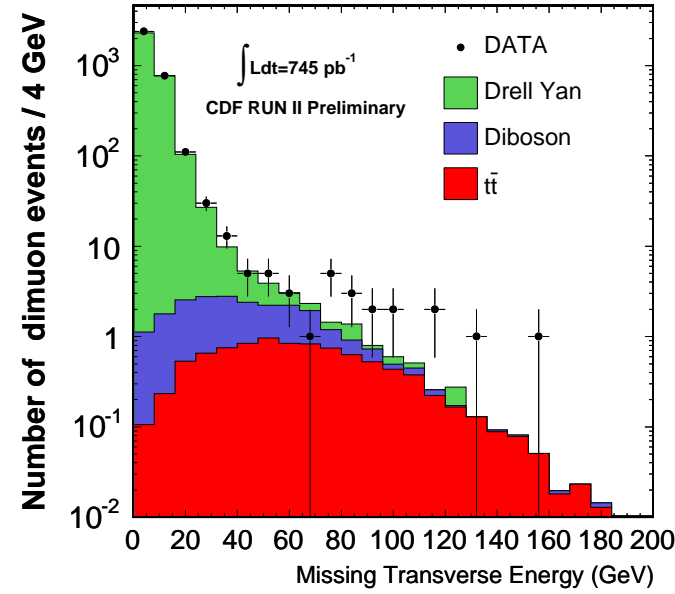

(a) Missing transverse energy

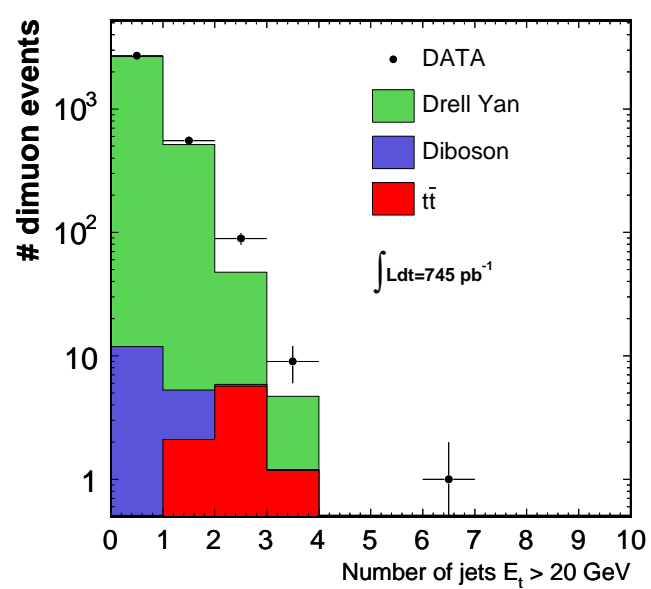

(b) Jet multiplicity

Figure 8.9. Kinematical distributions for dimuon events outside the $\mathrm{Z}$ mass window. 


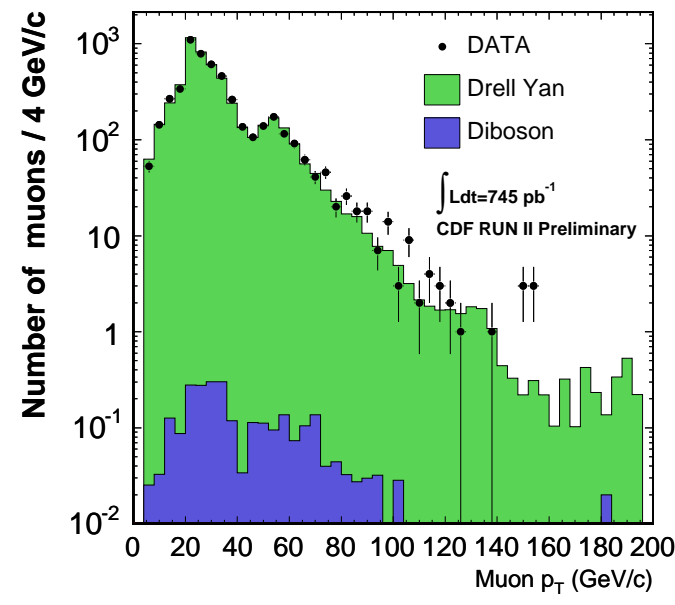

(a) Muon transverse momentum

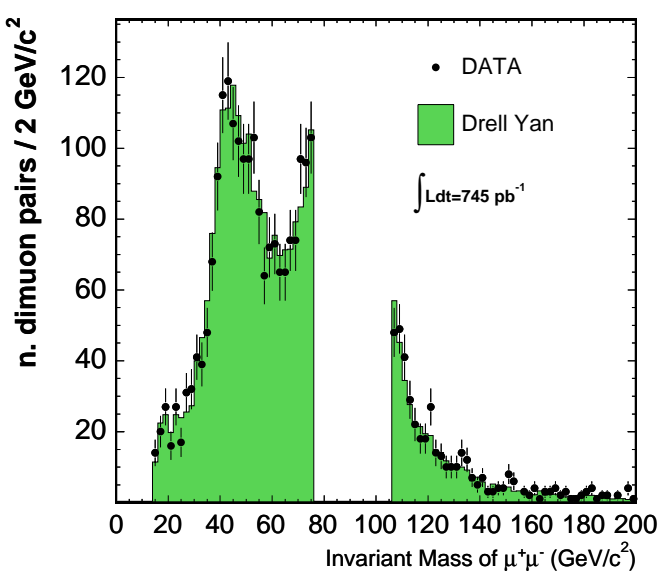

(b) Invariant mass

Figure 8.10. Control region G.

the number of events with opposite sign isolated muons ${ }^{6}$. We assume negligible $p_{T}$ and isolation dependences. Such contribution is affected by large systematic uncertainties. Furthermore, we might observe more events in data than in MC because of the uncertainty on the modeling of the underlying event and jet activity for off-shell $Z$ production. Jets from UE are typically low $E_{T}$ therefore with large opening angles $\Delta R$; the jet clustering algorithm could reconstruct two jets in place of a single jet. That the problem is not with the overall scaling of the predictions is confirmed by the good agreement obtained in control region $\mathrm{G}$ where the number of jets is small and the selection is not affected by jet and $\mathbb{E}_{\mathrm{T}}$ mismeasurement (Figure 8.10).

Finally we check our capability to predict the SM background in control region A whose most important distributions are shown in Figure 8.11. Control region A, dominated by Drell-Yan, includes contributions from diboson production and misidentified leptons; we also expect a small number of $t \bar{t}$ events. It is a crucial region since it re-

\footnotetext{
${ }^{6}$ The number of events with two same sign isolated muons is scaled up by ratio of the number of events with two non isolated opposite sign muons over the number of events with two non isolated same sign muons.
} 


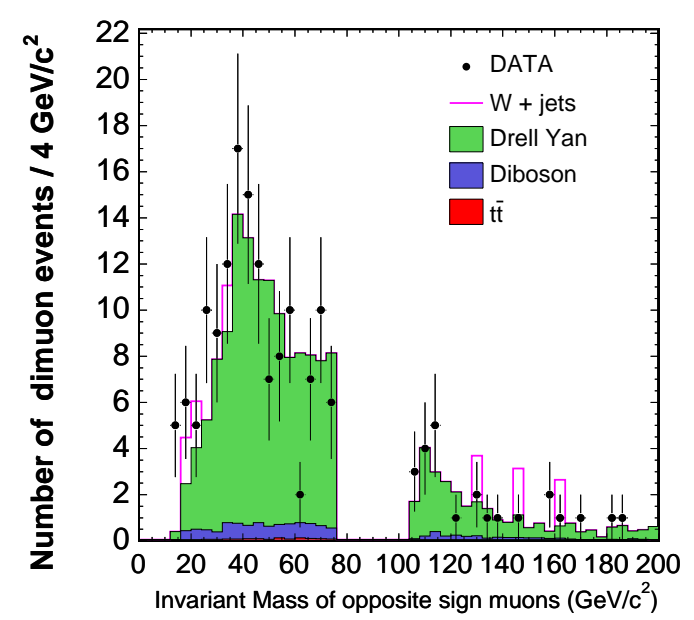

(a) Dimuon invariant mass.

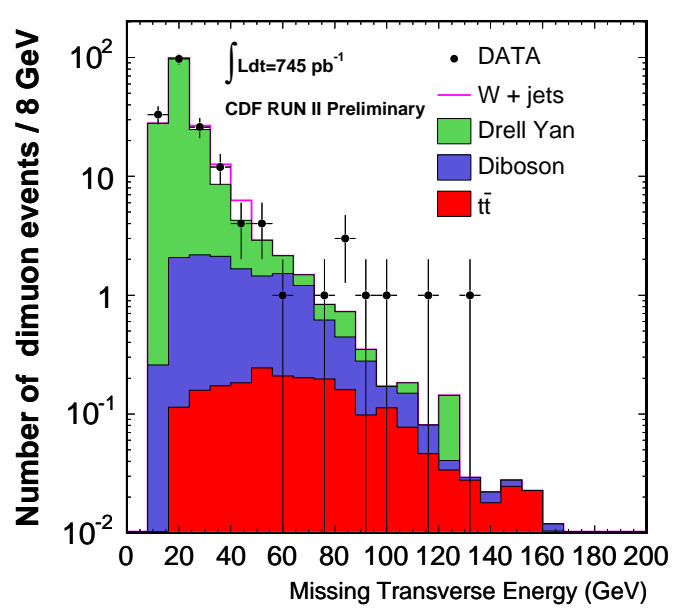

(b) Missing transverse energy.

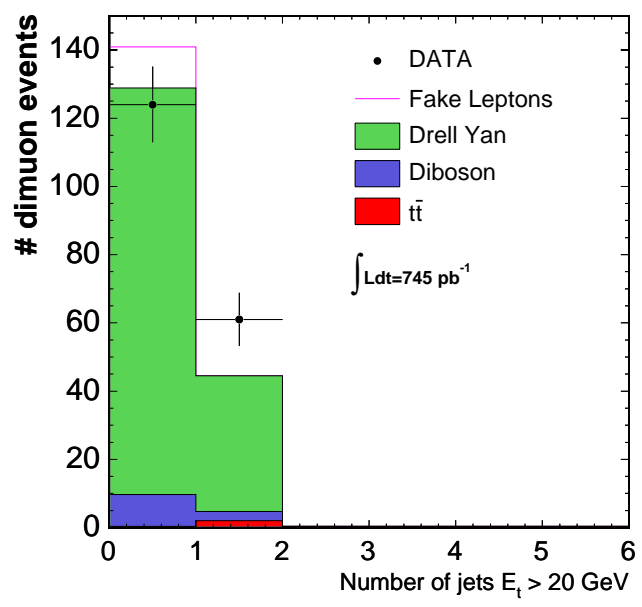

(c) Jet multiplicity

Figure 8.11. Control region A. 
quires the understanding of all SM backgrounds and contains the signal box. The excellent agreement between data and MC found, ensures the validity of our analysis. In Table 8.10 we summarize the results in all control regions with two muons.

Table 8.10

Comparison between the number of expected and observed events in events with two muons .

\begin{tabular}{|l|l|l|l|l|l|l|}
\hline CR & Diboson & HF & Drell-Yan & Fake leptons & TOTAL & DATA \\
\hline \hline A & $12.27 \pm 0.45$ & $2.13 \pm 0.07$ & $159 \pm 4$ & $12 \pm 6$ & $184 \pm 4$ & 185 \\
G & $1.30 \pm 0.14$ & $0.029 \pm 0.009$ & $2548 \pm 16$ & $2 \pm 1$ & $2552 \pm 16$ & 2531 \\
I & $1.54 \pm 0.09$ & $0.013 \pm 0.006$ & $15587 \pm 41$ & $<4.5$ & $15588 \pm 41$ & 15366 \\
E & $6.60 \pm 0.24$ & $0.54 \pm 0.04$ & $460 \pm 7$ & $<4.5$ & $468 \pm 7$ & 425 \\
A2 & $0.18 \pm 0.05$ & $6.77 \pm 0.13$ & $7.3 \pm 0.8$ & $1 \pm 0.5$ & $15 \pm 1$ & 26 \\
F & $0.17 \pm 0.04$ & $1.87 \pm 0.07$ & $29 \pm 2$ & $<4.5$ & $31 \pm 2$ & 31 \\
H & $0.03 \pm 0.02$ & $0.11 \pm 0.02$ & $23 \pm 2$ & $<4.5$ & $23 \pm 2$ & 38 \\
$\mathrm{~J}$ & $0.03 \pm 0.01$ & $0.03 \pm 0.01$ & $144 \pm 4$ & $<4.5$ & $144 \pm 4$ & 147 \\
$\mathrm{Z}$ & $9.14 \pm 0.27$ & $2.50 \pm 0.08$ & $18196 \pm 44$ & $<4.5$ & $18208 \pm 44$ & 17784 \\
\hline
\end{tabular}

\subsubsection{Control regions with one muon and one electron}

The comparison between the observed data and the predictions in the $\mu$-e channels is presented in Table 8.11 and Table 8.12. In the control region at low $\mathbb{E}_{\mathrm{T}}$, the dominant background is Drell-Yan (mostly $Z \rightarrow \tau \tau$ ), but in events with large missing transverse energy, we expect a large contribution from diboson production as well. Top pair production is the dominant background in high jet multiplicity regions, such as A2 and F. We observe good agreement in all control regions, as confirmed in Figure 8.12, where the Kinematical distributions are shown. The total number of events we observe in the $\mu$-CTE is 182 compared to a prediction of $181 \pm 2$ (stat). In the $\mu$-PLE/PHE, we expect $110 \pm 2$ (stat) and observe 118 . The $\mu$-e selection is affected 
by the calorimeter calibration, in particular at high instantaneous luminosity when the energy in neighboring towers can be uncorrelated from the electron. The excellent agreement also proves that we understand the forward tracking in detail.

Table 8.11

Comparison between the number of expected and observed events in events with one muon and one CTE.

\begin{tabular}{|l|l|l|l|l|l|l|}
\hline $\mathrm{CR}$ & Diboson & HF & Drell-Yan & Fake & TOTAL & DATA \\
\hline \hline $\mathrm{A}+\mathrm{E}$ & $29.7 \pm 2.2$ & $2.6 \pm 0.09$ & $47.7 \pm 2.2$ & $5.5 \pm 2.8$ & $85.5 \pm 3.1$ & 94 \\
$\mathrm{G}+\mathrm{I}$ & $1.6 \pm 0.5$ & $0.05 \pm 0.01$ & $50.6 \pm 2.2$ & $2.4 \pm 1.2$ & $54.7 \pm 2.3$ & 52 \\
$\mathrm{~A} 2+\mathrm{F}$ & $0.7 \pm 0.1$ & $8.4 \pm 0.2$ & $1.6 \pm 0.3$ & $1.0 \pm 0.5$ & $11.8 \pm 0.4$ & 7 \\
$\mathrm{~J}+\mathrm{H}$ & $0.004 \pm 0.002$ & $0.10 \pm 0.02$ & $0.2 \pm 0.1$ & $0.3 \pm 0.2$ & $0.7 \pm 0.1$ & 0 \\
\hline
\end{tabular}

Table 8.12

Comparison between the number of expected and observed events in events with one muon and one PLE/PHE.

\begin{tabular}{|l|l|l|l|l|l|l|}
\hline $\mathrm{CR}$ & Diboson & $\mathrm{HF}$ & Drell-Yan & Fake & TOTAL & DATA \\
\hline \hline $\mathrm{A}+\mathrm{E}$ & $16.1 \pm 1.3$ & $0.7 \pm 0.04$ & $28.2 \pm 1.5$ & $7 \pm 3.5$ & $52.6 \pm 1.5$ & 57 \\
$\mathrm{G}+\mathrm{I}$ & $0.4 \pm 0.1$ & $0.010 \pm 0.006$ & $28.6 \pm 1.4$ & $4 \pm 2$ & $33.9 \pm 1.4$ & 41 \\
$\mathrm{~A} 2+\mathrm{F}$ & $0.21 \pm 0.07$ & $2.2 \pm 0.08$ & $1.0 \pm 0.3$ & $2 \pm 1.0$ & $1.0 \pm 0.3$ & 3 \\
$\mathrm{~J}+\mathrm{H}$ & $0.02 \pm 0.02$ & $0.09 \pm 0.02$ & $0.2 \pm 0.1$ & $0.6 \pm 0.3$ & $0.2 \pm 0.1$ & 1 \\
\hline
\end{tabular}

\subsubsection{Control regions with three leptons}

In the $\mu-\mu$ channel the dominant background is Drell-Yan accompanied by a photon converting into $e^{+} e^{-}$or a misidentified lepton (the major contribution to fake events indeed originate from Drell-Yan with an additional lepton, the other SM processes being negligible). In the signal box, more than $60 \%$ of the background can be 


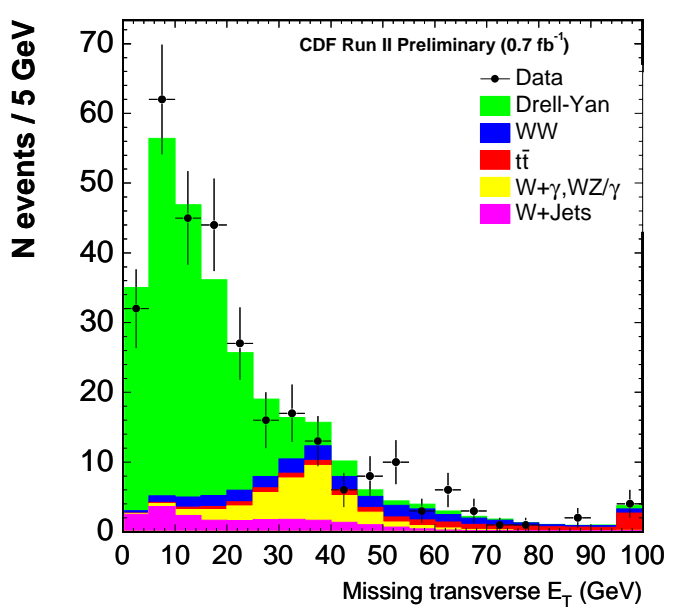

(a) Missing transverse energy.

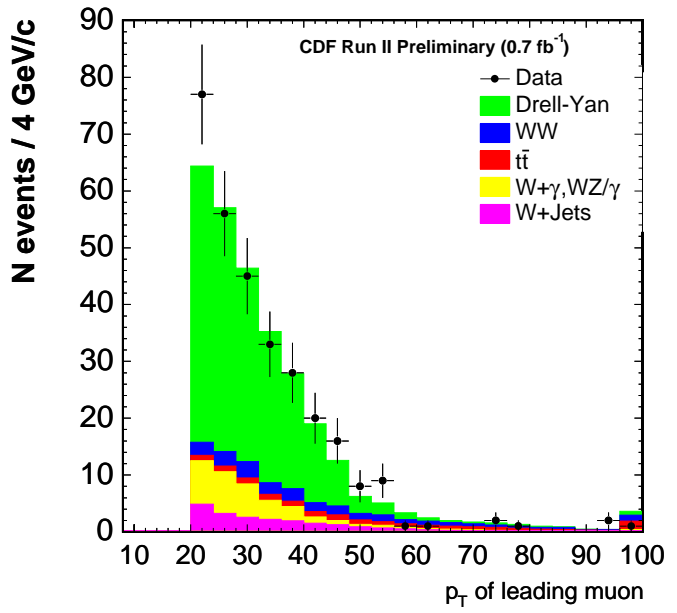

(b) Muon $p_{T}$.

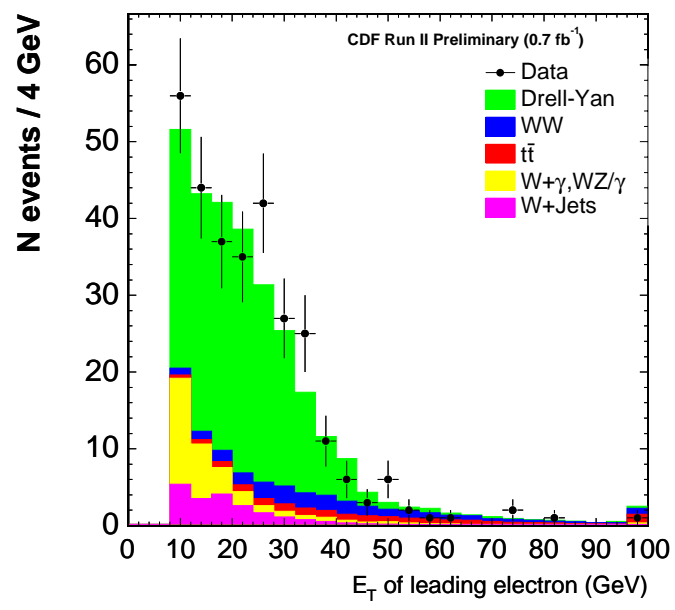

(c) Electron $E_{T}$.

Figure 8.12. Kinematical distributions in $\mu-\mathrm{e}$ events. 
Table 8.13

Comparison between the number of expected and observed events in events with two muon and one lepton.

\begin{tabular}{|l|l|l|l||l|l|}
\hline CR & DiBoson + HF & DY+Fake & Drell-Yan & TOTAL & DATA \\
\hline \hline A & $0.21 \pm 0.02$ & $0.2 \pm 0.1$ & $0.23 \pm 0.11$ & $0.64 \pm 0.11$ & BLIND \\
G & $0.060 \pm 0.008$ & $0.3 \pm 0.2$ & $3.9 \pm 0.5$ & $4.28 \pm 0.49$ & 4 \\
$\mathrm{I}$ & $0.37 \pm 0.02$ & $1.4 \pm 0.7$ & $6.3 \pm 0.7$ & $8.03 \pm 0.68$ & 6 \\
$\mathrm{E}$ & $1.13 \pm 0.04$ & $0.5 \pm 0.3$ & $0.5 \pm 0.2$ & $2.07 \pm 0.17$ & 2 \\
$\mathrm{~A} 2$ & $0.006 \pm 0.004$ & $0.03 \pm 0.02$ & $0.09 \pm 0.09$ & $0.12 \pm 0.09$ & 0 \\
$\mathrm{~F}$ & $0.012 \pm 0.004$ & $0.03 \pm 0.02$ & $<0.08$ & $0.04 \pm 0.01$ & 0 \\
$\mathrm{H}$ & $0.0012 \pm 0.0012$ & $0.01 \pm 0.01$ & $0.06 \pm 0.06$ & $0.07 \pm 0.06$ & 0 \\
$\mathrm{~J}$ & $0.006 \pm 0.003$ & $0.07 \pm 0.04$ & $0.24 \pm 0.14$ & $0.3 \pm 0.1$ & 0 \\
$\mathrm{Z}$ & $1.65 \pm 0.05$ & $2.3 \pm 1.2$ & $8.2 \pm 0.8$ & $12.2 \pm 0.8$ & 8 \\
\hline
\end{tabular}

associated to Drell-Yan with $\sim 30 \%$ contribution from diboson production. $W Z / \gamma^{*}$ events dominate in control region $\mathrm{E}$ because the $Z$ mass is shifted due to the photon radiation. The details of the comparison between data and $\mathrm{MC}$ can be found in Table 8.13. Similarly to the $\mu-\mu$ channel, the control regions of the $\mu$-e selection exhibit good agreement shown in Table 8.14 and Table 8.15. If the number of predicted events for a given MC based background is 0, we place an upper limit at 95\%C.L. [16] calculated as $N_{\text {exp }}<\frac{\epsilon_{D a t a}^{a v}}{\epsilon_{M C}^{a v}} \times \mathcal{L} \times \frac{\sqrt{3}}{\mathcal{L}^{\prime}}$ where $\frac{\epsilon_{D a t a}^{a v}}{\epsilon_{M C}^{a v}}$ is the average scale factor per event, $\mathcal{L}$ and $\mathcal{L}^{\prime}$ are respectively the luminosity of the data and generated MC sample.

In summary we show a good understanding of the Standard Model backgrounds in all control regions we explored. Therefore we are confident in taking the last step in our search which is counting the number of data events in the signal boxes. 
Table 8.14

Comparison between the number of expected and observed events in events with one muon, one CTE and one lepton.

\begin{tabular}{|l|l|l|l|l|l|l|}
\hline $\mathrm{CR}$ & Diboson & HF & Drell-Yan & Fake & TOTAL & DATA \\
\hline \hline $\mathrm{G}+\mathrm{I}$ & $0.020 \pm 0.005$ & $<0.005$ & $4.2 \pm 0.4$ & $0.05 \pm 0.03$ & $4.3 \pm 0.4$ & 3 \\
$\mathrm{~J}+\mathrm{H}$ & $<0.003$ & $<0.005$ & $<0.07$ & $0.02 \pm 0.01$ & $0.02 \pm 0.01$ & 0 \\
\hline
\end{tabular}

Table 8.15

Comparison between the number of expected and observed events in events with one muon, one PLE/PHE and one lepton.

\begin{tabular}{|l|l|l|l|l|l|l|}
\hline CR & Diboson & HF & Drell-Yan & Fake & TOTAL & DATA \\
\hline \hline $\mathrm{G}+\mathrm{I}$ & $0.01 \pm 0.005$ & $<0.005$ & $1.6 \pm 0.2$ & $0.02 \pm 0.01$ & $1.6 \pm 0.2$ & 0 \\
$\mathrm{~J}+\mathrm{H}$ & $<0.003$ & $<0.005$ & $<0.07$ & $0.001 \pm 0.0005$ & $0.001 \pm 0.0005$ & 0 \\
\hline
\end{tabular}




\section{Discussion of the results and conclusions}

We performed a blind counting experiment searching for the associated production of chargino and neutralino in events with at least three leptons and significant missing transverse energy. In this Chapter we present the results of our analysis and its interpretation in two mSUGRA scenarios.

\subsection{Systematic uncertainties}

There are several systematic uncertainties which affect the number of predicted events $N_{\text {exp }}$, and, consequently the interpretation of the search result. If the uncertainty obeys a Gaussian probability function, we can measure the variation of $N_{\text {exp }}$ due to a given uncertainty as

$$
\delta_{N_{e x p}}=\frac{1}{2} \frac{N_{e x p}^{+1 \sigma}-N_{e x p}^{-1 \sigma}}{N_{e x p}}
$$

where $\sigma$ is the width of the Gaussian determined in subsidiary measurements, if not specified. The effect of each systematic uncertainty is calculated separately. In particular, we consider the following systematic uncertainties.

- Luminosity. At CDF, the integrated luminosity is measured with an accuracy of $6 \%$ [48]. The MC based backgrounds are scaled accordingly and the uncertainty calculated using Equation 9.1.

- Parton Distribution Function. The cross section and the event kinematics depend on the momentum of the incoming partons, whose probability is parametrized in "parton distribution functions" (PDF's). The PDF's are determined from a fit to data from a number of experiments [75] [76]. The fit result is associated to systematic uncertainties which propagate into our determination of the number 
of expected events. In particular for the PDFs from the CTEQ group [75], the uncertainties are represented by a set of 40 eigenvectors. To calculate the uncertainty $\delta_{N_{e x p}}$, each Monte Carlo event is scaled according to the variation of the PDF from the central value. The variations are then added in quadrature and used to obtain the number of expected events in Equation 9.1.

- Initial and Final State Radiation (ISR/FSR). At high order QCD, the event might contain jets from gluon radiation. The ISR and FSR are modeled in the Monte Carlo and are subject to the uncertainty of the parton shower model. A conservative approach is taken when we calculate $\delta_{N_{e x p}}=\frac{1}{2} \frac{N_{e x p}^{M C 1}-N_{e x p}^{M C 2}}{N_{e x p}}$ where $N_{\text {exp }}^{M C 1}$ and $N_{e x p}^{M C 2}$ are the SM predictions calculated in Monte Carlo samples with different ISR/FSR contents ${ }^{1}$.

- Jet Energy Scale. The jet energy resolution affects the number of events via the jet multiplicity and the correction of the $E_{\mathrm{T}}$ for mismeasured jets. The jet energy scale is varied within its uncertainty as in [65] and the number of events recalculated and used in Equation 9.1.

- Lepton Identification Scaling Factors. As mentioned in Section 6.3, the identification efficiency in MC differs from the one in data therefore the lepton detection probability needs to be corrected in the MC events. We vary the scale factors within their uncertainty and determined the number of expected events used in Equation 9.1.

- Lepton misidentification rate. The probability to fake a lepton is measured in jet data as described in Section 7.4. The uncertainty is determined as the maximum variation among the different data samples used. A conservative uncertainty of $50 \%$ is quoted. The number of fake events is scaled by this amount.

- Photon Conversion Removal. Similarly to the misidentification rate uncertainty, the systematic due to the photon tagging efficiency is measured scaling the total

${ }^{1}$ In particular, the parameters set in PYTHIA are the following: $\operatorname{PARP}(61)=0.292 \mathrm{GeV}$, $\operatorname{PARP}(64)=0.5$ and $\operatorname{PARP}(61)=0.073 \mathrm{GeV}, \operatorname{PARP}(64)=2.0$. 
number of MC events containing a photon by the uncertainty on the scale factor measured in Appendix C.

- Production Cross Section. The uncertainty on the production cross section influences the number of SM and SUSY signal events we predict in the different kinematic regions. We vary the NLO cross sections by the known theoretical uncertainty quoted in Table 9.1 for the different processes. For the SUSY production cross section, the uncertainty is calculated as the difference between the rescaled LO cross section calculated with PYTHIA [67] and the cross section determined using Prospino [19]. For the Drell-Yan and Diboson production, the uncertainty is determined calculating the cross section with MCFM [77]. For the top pair production we refer to theoretical calculation in [70].

Table 9.1

Systematic uncertainties on the NLO production cross sections.

\begin{tabular}{|c|c|}
\hline Process & Uncertainty (\%) \\
\hline \hline Drell-Yan & 8 \\
Diboson & 5 \\
$t \bar{t}$ & 10 \\
SUSY & 7 \\
\hline
\end{tabular}

The impact of each systematic uncertainty on the SUSY signal and the SM background is listed in Table 9.2 to Table 9.4.

\subsection{Data in the signal box}

The results of the three independent searches we carried out in this dissertation are presented in Table 9.5. We observe one event in the $\mu-\mu$ channel, which is compatible with the SM expectations. In this event we reconstruct three vertices, 
Table 9.2

Systematic uncertainties in the $\mu-\mu$ channel.

\begin{tabular}{|c|c|c|}
\hline Systematic Uncertainty & SUSY Signal (\%) & SM Background(\%) \\
\hline \hline Luminosity & 6 & 4 \\
PDF & 2 & 2 \\
ISR & 2 & 4 \\
Jet Energy Scale & 3 & 4 \\
Muon ID & 5 & 4 \\
Lepton fake rate & NA & 16 \\
Conversion removal & NA & 10 \\
Cross Section & 5 & 7 \\
\hline
\end{tabular}

Table 9.3

Systematic uncertainties in the $\mu-\mathrm{CLE} / \mathrm{CTE}$ channel.

\begin{tabular}{|c|c|c|}
\hline Systematic Uncertainty & SUSY Signal (\%) & SM Background(\%) \\
\hline \hline Luminosity & 6 & 6 \\
PDF & 2 & 2 \\
ISR & 2 & 4 \\
Jet Energy Scale & 0.3 & 7 \\
Electron ID & 2 & 4 \\
Muon ID & 7 & 4 \\
Lepton fake rate & NA & 13 \\
Conversion removal & NA & 7 \\
Cross Section & 4 & 7 \\
\hline
\end{tabular}

at $Z=30$ (primary vertex with a raw total transverse energy of $120 \mathrm{GeV}$ ), $Z=7$ and $Z=-29 \mathrm{~cm}$, respectively. Three muons and one jet originate from the primary vertex. The leading muon is a CMX muon which fired the trigger, the next to leading 
Table 9.4

Systematic uncertainties in the $\mu-$ PLE/PHE channel.

\begin{tabular}{|c|c|c|}
\hline Systematic Uncertainty & SUSY Signal (\%) & SM Background(\%) \\
\hline \hline Luminosity & 6 & 6 \\
PDF & 2 & 2 \\
ISR & 2 & 4 \\
Jet Energy Scale & $<0.1$ & 2 \\
Electron ID & 5 & 14 \\
Muon ID & 5 & 4 \\
Lepton fake rate & NA & 8 \\
Cross Section & 7 & 7 \\
\hline
\end{tabular}

Table 9.5

Number of expected and observed events in the the three analysis channels.

\begin{tabular}{|c|c|c|c|}
\hline Channel & SM background & SUSY Benchmark point & Data \\
\hline \hline$\mu-\mu$ & $0.64 \pm 0.11($ stat $) \pm 0.14($ sys $)$ & $1.6 \pm 0.1($ stat $) \pm 0.18($ sys $)$ & 1 \\
$\mu-\mathrm{CTE}$ & $0.42 \pm 0.05($ stat $) \pm 0.07($ sys $)$ & $0.83 \pm 0.06($ stat $) \pm 0.10($ sys $)$ & 0 \\
$\mu-\mathrm{PLE} / \mathrm{PHE}$ & $0.36 \pm 0.06($ stat $) \pm 0.05($ sys $)$ & $0.20 \pm 0.02($ stat $) \pm 0.02($ sys $)$ & 0 \\
\hline
\end{tabular}

muon (back to back with respect to the leading muon) is a CMIO muon with invariant mass of $72 \mathrm{GeV} / c^{2}$ with the leading one. The third muon is a CMUP muon. All muons have good tracks with silicon hits attached. An additional electron candidate is reconstructed from the vertex at $Z=7 \mathrm{~cm}$ but it fails the energy and isolation requirements. The transverse mass of the third muon and $E_{\mathrm{T}}$ is $7 \mathrm{GeV}$. Besides the leptons, a non b-tagged jet with corrected $\mathrm{E}_{T}>20 \mathrm{GeV}$ originated from the same interaction. The missing transverse energy is just above the threshold of our selection 
with a value of $15.5 \mathrm{GeV}$. More details can be found in Table 9.6 and Figure 9.1 and Figure 9.2 show the $r \phi$ and $Z$ views.

Table 9.6

Description the event observed in the $\mu-\mu$ channel.

\begin{tabular}{|c|c|}
\hline Leading lepton & $\mathrm{CMX} \mu^{-}, \mathrm{p}_{T}=52 \mathrm{GeV}, \eta=-0.9, \phi=-0.26, \mathrm{Z}_{0}=30 \mathrm{~cm}$ \\
\hline Next Leading lepton & $\mathrm{CMIO} \mu^{+}, \mathrm{p}_{T}=27 \mathrm{GeV}, \eta=-0.5, \phi=2.33, \mathrm{Z}_{0}=30 \mathrm{~cm}$ \\
\hline Third lepton & $\operatorname{CMUP} \mu^{-}, \mathrm{p}_{T}=8 \mathrm{GeV}, \eta=-0.16, \phi=0.79, \mathrm{Z}_{0}=30 \mathrm{~cm}$ \\
\hline Other leptons & Electron candidate $\mathrm{E}_{T} 4.3 \mathrm{GeV}, \eta=-0.2, \phi=0.411, \mathrm{Z}_{0}=7 \mathrm{~cm}$ \\
\hline Missing Transverse Energy & $15.5 \mathrm{GeV}, \phi=1.42$ \\
\hline Vertices & $Z_{1}=31 \mathrm{~cm}, Z_{2}=7 \mathrm{~cm}$, vertex $Z_{3}=-29 \mathrm{~cm}$ \\
\hline N. Jets L5 $\mathrm{E}_{T}>20 \mathrm{GeV}$ & 1 \\
\hline Leading jet & $\mathrm{L} 5 \mathrm{E}_{T}=47 \mathrm{GeV}, \eta=-0.13, \phi=-0.279, Z_{0}=19.4 \mathrm{~cm}$ \\
\hline Invariant mass of OS Muons & $\mathrm{m}_{C M X-C M I O}=72.5 \mathrm{GeV}$ \\
\hline Transverse mass & $m_{T(C M U P-M E T)}=7 \mathrm{GeV}$ \\
\hline
\end{tabular}




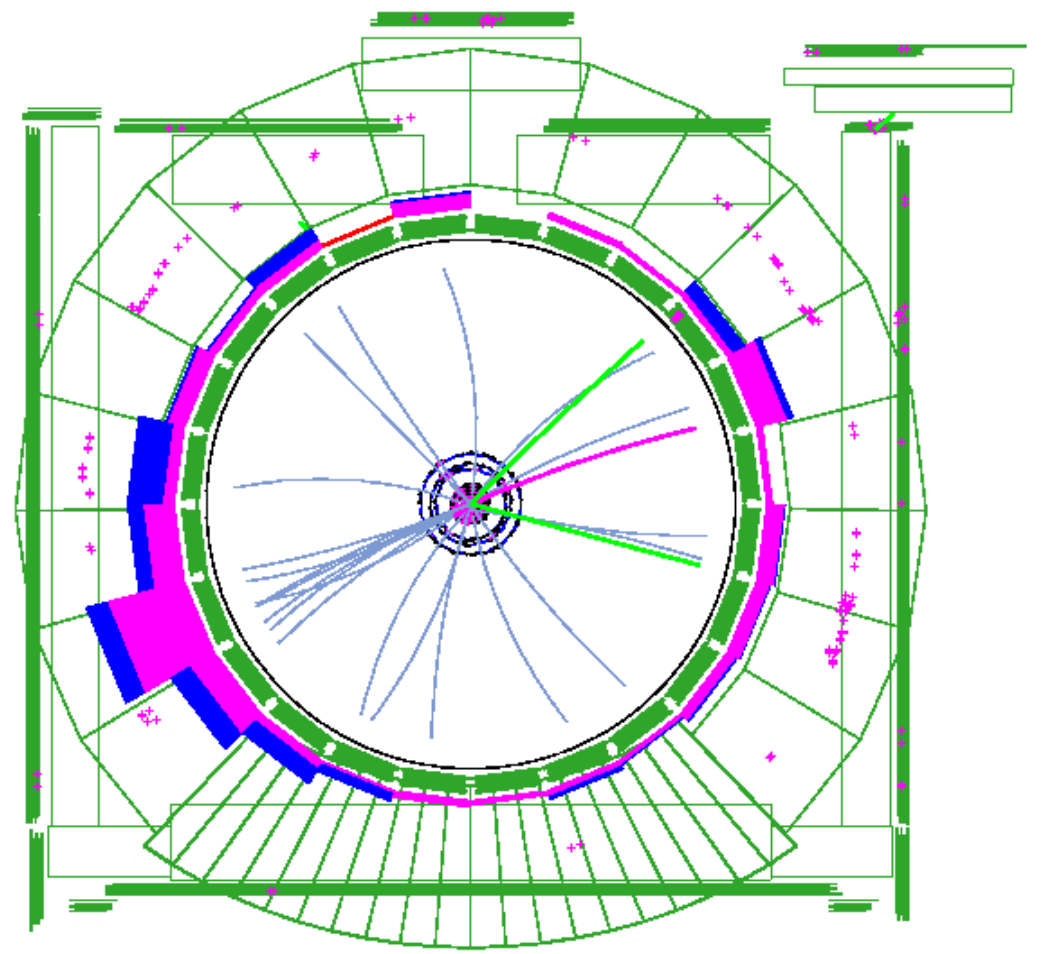

Figure 9.1. $r \phi$ view. A cut of $p_{T} \geq 1 \mathrm{GeV}$ is applied to the tracks in the event. 


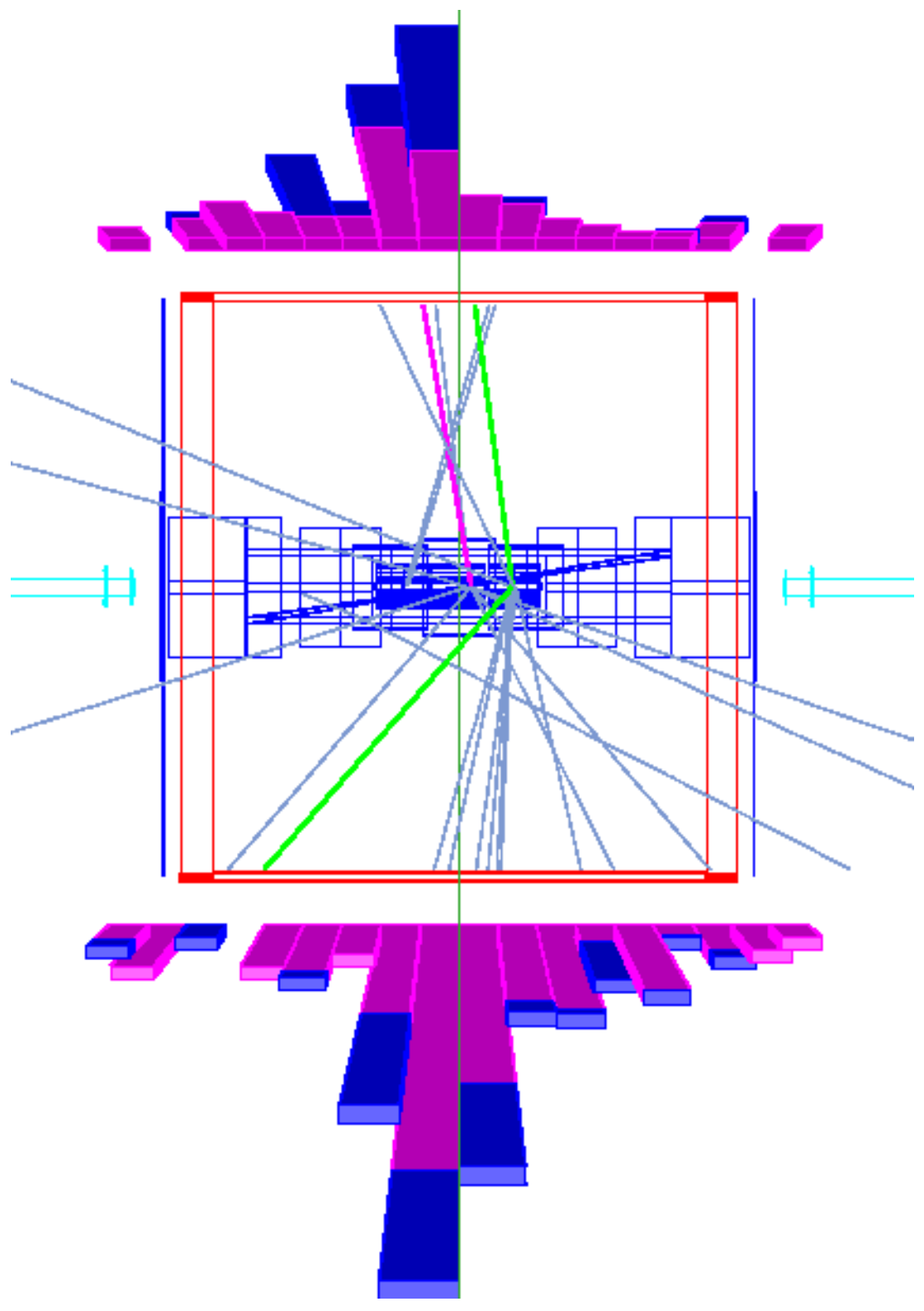

Figure 9.2. $\mathrm{Z}$ view. A cut of $p_{T} \geq 1 \mathrm{GeV}$ is applied to tracks in the event. 


\subsection{Interpretation of the results}

In Section 9.2 we showed that the number of observed events agrees with the expectations from the SM backgrounds. Therefore we would like to determine an upper limit on the number of SUSY events present in the data.

The probability of observing $n_{0}$ events depends on the mean number of expected SUSY events $\mu_{s}$ in absence of background according to the Poisson statistics [78]:

$$
\mathcal{P}\left(n_{0} \mid \mu_{s}\right)=\frac{\mu_{s}^{n_{0}} e^{-\mu_{s}}}{n_{0} !}
$$

We want to calculate the maximum value $\mathrm{N}$ of $\mu_{s}$ such that the observed $n_{0}$ is compatible to $\mathrm{N}$ at $95 \%$. We could rephrase it, by saying that we calculate the value of $\mu_{s}$ such that the probability $\epsilon$ of observing less than $n_{0}$ events is equal or less than $5 \%$. Therefore we can apply Equation 9.2 and obtain

$$
\epsilon=\sum_{n=0}^{n=n_{0}} \mathcal{P}\left(n \mid \mu_{s}\right) .
$$

If the number of expected SUSY events exceeds $\mathrm{N}$, we can claim that the SUSY process is excluded at $(1-\epsilon) \%$. As an example we assume $n_{0}=0$ and calculate $\mathrm{N}$ at $95 \%$ as $\epsilon=0.05=\sum_{n=0}^{n=n_{0}} \frac{\mu_{s}^{n_{0}} e^{-\mu_{s}}}{n_{0} !}=e^{-\mu_{s}}$ resulting in $\mathrm{N}=3$. It means that an observation of 0 events would be compatible with 3 events at 95\%. However, we should take into account possible sources of background and modify Equation 9.3 into

$$
\epsilon=\frac{\sum_{n=0}^{n=n_{0}} \mathcal{P}\left(n \mid \mu_{s}+b\right)}{\sum_{n=0}^{n=n_{0}} \mathcal{P}(n \mid b)} .
$$

The value of $\mathrm{N}$ we obtain is the number of SUSY events contained in the data, along with $b$ background events (assumed known precisely), such that the probability of observing less than $n_{0}$ is $\epsilon$ and $b \leq n_{0}$ [79]. The upper limit decreases with increasing background but the sensitivity becomes worse $^{2}$. In particular, if we observe 0 , the limit is independent of the background.

In practice we don't know the number of background events precisely, and we rather

${ }^{2}$ It is due to the fact that the mean limit increases with the increase in the background. 
associate an uncertainty $\sigma_{b}$ with our knowledge of the background. The uncertainties, in particular if correlated, typically weaken the limit. To properly include the uncertainties, we calculate the upper limit generalized to three channels in a purely Bayesian approach.

We build a joint likelihood from the product of the individual channel likelihoods as

$$
\mathcal{L}\left(\bar{n} \mid \sigma_{S U S Y}, \bar{b}, \bar{\epsilon}\right)=\prod_{i=1}^{3} \frac{\mu_{i}^{n_{i}} e^{-\mu_{i}}}{n_{i} !}
$$

where the expected number of events per channel is $\mu_{i}=\mu_{i, S U S Y}+b_{i}=L_{i} \times \sigma_{i} \times \epsilon_{i}+b_{i}$ ( $L_{i}$ is the integrated luminosity, $\sigma_{i}$ the SUSY cross section, $\epsilon_{i}$ its acceptance and $b_{i}$ the background). In a Bayesian approach, we treat $\sigma_{i}$ as an unknown variable with a prior probability distribution (simply called prior); the prior expresses the uncertainty on the quantity before it is measured. If we apply the Bayes theorem, and we multiply the prior by the joint likelihood, we get the posterior probability distribution, which is the conditional $^{3}$ distribution of the uncertain quantity once the result of the measurement is known. Therefore we can write,

$$
\mathcal{P}\left(\sigma_{S U S Y} \mid \bar{n}, \bar{b}, \bar{\epsilon}\right)=k \times \mathcal{L}\left(\bar{n} \mid \sigma_{S U S Y}, \bar{b}, \bar{\epsilon}\right) \times \mathcal{P}\left(\sigma_{S U S Y}\right)
$$

where $\mathrm{k}$ is a normalization factor. The choice of the prior $\mathcal{P}\left(\sigma_{S U S Y}\right)$ is a subjective assessment, for instance pre-existing evidence could lead to a specific choice. In our case we assume total ignorance (uninformed prior) and we set $\mathcal{P}\left(\sigma_{S U S Y}\right)=0$ if $\sigma_{S U S Y}<0$ and $\mathcal{P}\left(\sigma_{S U S Y}\right)=1$ otherwise. The upper limit at $95 \%$ credibility level, or simply $95 \%$ C.L., is then calculated as,

$$
\int_{0}^{\sigma_{95}} \mathcal{P}\left(\sigma_{S U S Y}^{\prime} \mid \bar{n}, \bar{b}, \bar{\epsilon}\right) d \sigma_{S U S Y}^{\prime}=0.95 .
$$

The predictions are subject to uncertainties, both on the SUSY signal and the SM background. These are reproduced forcing the upper limit we just calculated to vary according to a Gaussian distribution (in the assumption that the uncertainties do obey ${ }^{3}$ The conditional probability is the probability of an event A given the occurance of some other event B. 
such statistics). The uncertainties are usually called "nuisance parameters" [80], and they are parametrized in terms of a prior distribution (typically a Gaussian which is the posterior from subsidiary measurements) to be marginalized ${ }^{4}$. For example we can write the likelihood of a single channel where the acceptance of the SUSY signal is associated to an uncertainty $\sigma_{\epsilon}$,

$$
\mathcal{L}\left(n \mid \sigma_{S U S Y}, b, \epsilon\right)=\frac{1}{\sqrt{2 \pi \sigma_{\epsilon}^{2}}} \int_{0}^{\infty} \frac{\mu^{\prime n} e^{-\mu^{\prime}}}{n !} e^{-\frac{\left(\epsilon^{\prime}-\epsilon\right)^{2}}{2 \sigma_{\epsilon}^{2}}} d \epsilon^{\prime} .
$$

Different techniques are utilized to solve these integrals. At CDF we exploit a Monte Carlo method. We generate a finite prior-ensemble which replaces the continuous joint nuisance prior and we calculate an average. In the previous case, it means having a a set of numbers drawn from a Gaussian distribution for the uncertainty on the acceptance,

$$
\mathcal{L}\left(n \mid \sigma_{S U S Y}, b, \epsilon\right)=\frac{1}{M} \sum_{k=1}^{M} \frac{\mu_{k}^{\prime n} e^{-\mu_{k}^{\prime}}}{n !}
$$

where $\mu_{k}^{\prime}=L \times \sigma^{\prime} \times \epsilon_{k}+b$ (the values $\epsilon_{k}$ are drawn from a Gaussian with width $\sigma_{\epsilon}$.). The accuracy of the method goes as $\frac{1}{M}$ where $M$ is the number of Monte Carlo numbers generated. The procedure can be extended to several systematic uncertainties, taking into account the correlations. Once the marginalized likelihood is calculated, it can be converted into a posterior density for the SUSY signal.

We combine the three channels $\mu-\mu, \mu-\mathrm{CTE}$ and $\mu-\mathrm{PLE} / \mathrm{PHE}$ in an exclusive way, taking care of possible overlaps in the event selection. The systematic uncertainties are included in the estimate of the upper limit according to the correlation between channels reported in Table 9.7. The upper limit on the $\sigma \times B R$ is calculated as a function of the chargino mass along a benchmark line in the "mSUGRA like" scenario described in Section 8.2 and shown in Figure 9.3. The limit is slightly above the NLO effective cross section. This indicates that the three analyses alone are almost sensitive to the previously excluded associated production of chargino and

${ }^{4}$ The marginal probability is the probability of one event regardless of the other event. It is obtained by summing over, or integrating, the joint probability over the unrequired events. 
Table 9.7

Correlation between the systematic uncertainties among the different channels.

\begin{tabular}{|c|c|}
\hline Systematic Uncertainty & Correlation (\%) \\
\hline \hline Luminosity & 100 \\
PDF & 100 \\
ISR & 100 \\
Jet Energy Scale & 100 \\
Electron ID & 100 \\
Muon ID & 100 \\
Lepton fake rate & 100 \\
Conversion removal & 0 \\
SM Cross Section & 100 \\
\hline
\end{tabular}

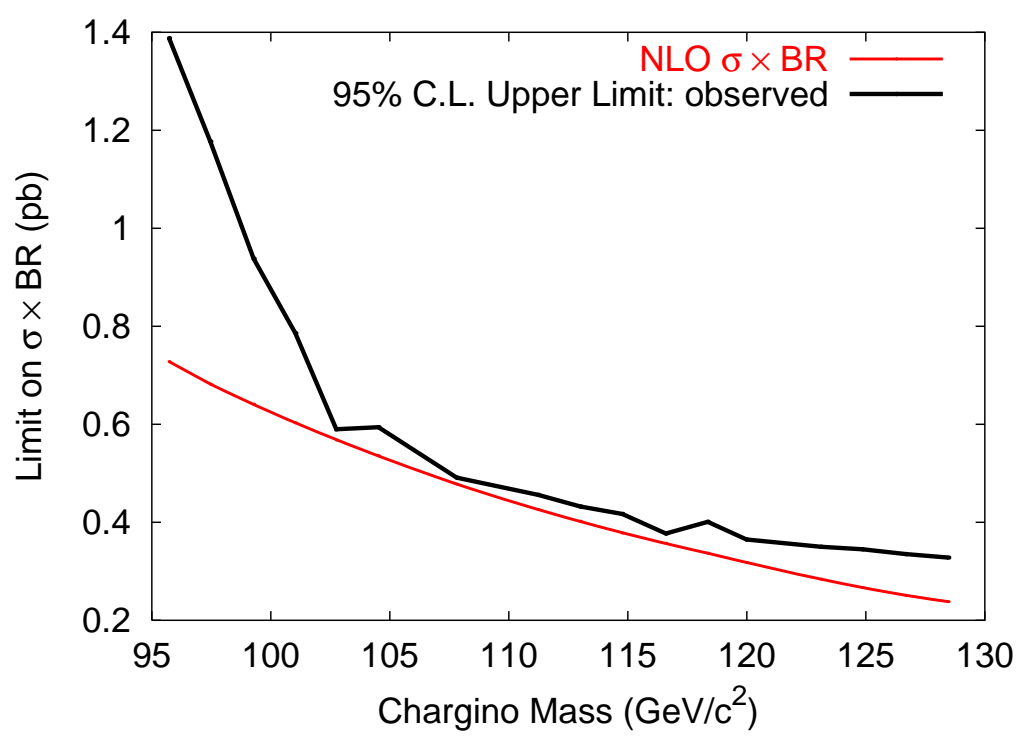

Figure 9.3. The excluded cross section limit is plotted as a function of the chargino mass in the mSUGRA like scenario.

neutralino. The huge impact of the statistical uncertainty can be inferred from Fig- 
ure 9.4 where we present the upper limit in the assumption of null observation. In



Figure 9.4. The excluded cross section limit is plotted as a function of the chargino mass in the mSUGRA like scenario with the assumption of observing 0 events (dashed line).

Figure 9.5 we compare the combined upper limit to the one obtained from the $\mu-\mu$ analysis only. Since both the expected number of signal and background events are small and few candidate events are observed in the data, the ability to exclude a SUSY signal at some level improves significantly by combining several searches. The combination is crucial if the sensitivity is limited by the luminosity and not by the kinematic boundary. Furthermore, combining complementary channels provides an exclusion for several values of the model parameters.

\subsection{Results of the searches for chargino and neutralino at CDF}

The CDF Collaboration pursued the search for SUSY in six additional channels for maximizing the acceptance. The strategy is similar to the analyses presented in this dissertation, but different data sets and third lepton requirements ensure the desired improvements. Events are selected if the $\mathbb{E}_{\mathrm{T}}$ is larger than $15 \mathrm{GeV}$ and two 


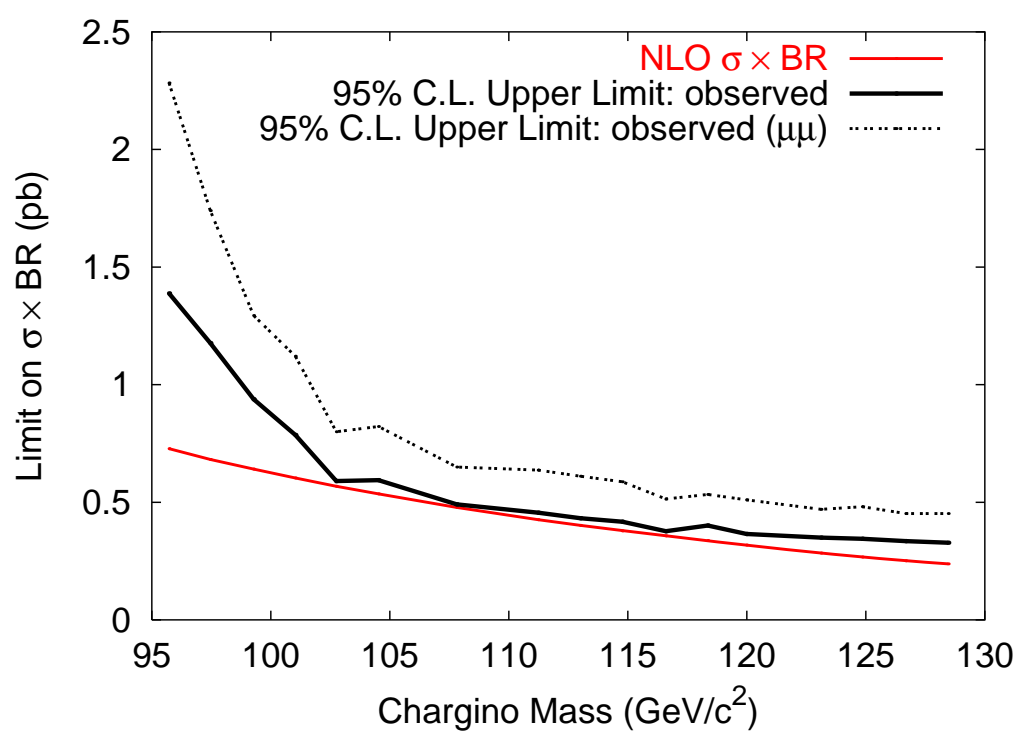

Figure 9.5. The excluded cross section limit is plotted as a function of the chargino mass in the mSUGRA like scenario combining the three analysis (solid line) and for the $\mu-\mu$ channel only (dashed line).

isolated leptons satisfy the identification criteria. In two channels, CDF requires at least three fully reconstructed and identified leptons. eel events are explored in data collected with the high $E_{T}$ single electron trigger [81] and $\mu \mu \ell$ are selected from a low $p_{T}$ dilepton data-sample [82]. A good sensitivity to hadronic decays of $\tau \mathrm{s}$ is provided by a search carried out in the low $p_{T}$ dilepton data-sample where CDF asks for two isolated electrons and an additional isolated track [83]. For sake of simplicity we refer to these analyses as trilepton searches. The trilepton analyses are summarized in Table 9.8 and the systematic uncertainties shown in Table 9.9.

Finally CDF explores both electron and muon high $p_{T}$ data samples relaxing the requirement on the third object and asking only for two high $p_{T}$ like sign leptons [84] (dilepton $L S$ search). Details can be found in Table 9.10 and Table 9.11. The LS analysis is the most sensitive at low chargino and neutralino mass where one lepton might be too low $p_{T}$ to be reconstructed, and similarly in leptonic $\tau$ decays. The acceptance as a function of the chargino mass is illustrated in Figure 9.6 for the 
Table 9.8

Trilepton analyses; left column, eel selection in high $E_{T}$ single electron data sample; middle column, $\mu \mu \ell$ in low $p_{T}$ dilepton data sample; left column, ee + track selection in low $p_{T}$ dilepton data sample.

\begin{tabular}{|c|c|c|c|}
\hline & $e e \ell$ & $\mu \mu \ell$ low $p_{T}$ & $e e+$ track \\
\hline \hline Luminosity $\left(\mathrm{pb}^{-1}\right)$ & 346 & 312 & 607 \\
SUSY Signal & $0.49 \pm 0.06$ & $0.17 \pm 0.04$ & $0.92 \pm 0.09$ \\
SM Background & $0.17 \pm 0.05$ & $0.13 \pm 0.03$ & $0.49 \pm 0.10$ \\
Number of observed events & 0 & 0 & 1 \\
\hline
\end{tabular}

Table 9.9

Systematic uncertainties of the trilepton analyses (the first number is the SUSY signal, the second the SM background).

\begin{tabular}{|c|c|c|c|}
\hline & $e e \ell$ & $\mu \mu \ell$ low $p_{T}$ & $e e+$ track \\
\hline \hline Luminosity & 6,4 & 6,1 & 6,6 \\
PDF & 2,2 & NA, NA & 2,2 \\
ISR/FSR & 4,4 & NA, NA & 5,11 \\
Jet Energy Scale & 1,22 & NA, NA & 1,17 \\
Electron ID & 3,3 & 6,1 & 3,3 \\
Electron Energy Scale & 3,3 & NA, NA & 3,3 \\
Muon ID & $0.7,0.2$ & 6,1 & NA, NA \\
Muon Isolation Criteria & $0.7,0.1$ & 12,2 & NA, NA \\
Fake Rate & NA, & NA, 21 & NA, 22 \\
Trigger Efficiency & NA, NA & $0.6,0.1$ & NA, 3 \\
Conversion Removal & NA, 0.7 & NA, NA & NA, NA \\
Flavor Background Estimated & NA, NA & NA, 6 & NA, NA \\
Theory Cross Section & 7,7 & NA, NA & 7,7 \\
\hline
\end{tabular}


"mSUGRA like" scenario. Each analysis has slightly different preselection cuts to

Table 9.10

LS analyses: left column $e^{ \pm} e^{ \pm}$selection in high $E_{T}$ single electron data sample; middle column, $\mu^{ \pm} \mu^{ \pm}$in high $p_{T}$ single muon data sample; left column, $e^{ \pm} \mu^{ \pm}$selection in high $E_{T}$ single electron data sample and high $p_{T}$ single muon data sample.

\begin{tabular}{|c|c|c|c|}
\hline & $e e \mathrm{LS}$ & $\mu \mu \mathrm{LS}$ & $\mu e \mathrm{LS}$ \\
\hline \hline Luminosity $\left(\mathrm{pb}^{-1}\right)$ & 704 & 704 & 704 \\
SUSY Signal & $0.64 \pm 0.07$ & $0.91 \pm 0.10$ & $1.63 \pm 0.16$ \\
SM Background & $2.60 \pm 0.39$ & $0.73 \pm 0.08$ & $3.50 \pm 0.60$ \\
Number of observed events & 4 & 0 & 5 \\
\hline
\end{tabular}

Table 9.11

Systematic uncertainties of the LS analyses (the first number is the SUSY signal, the second the SM background).

\begin{tabular}{|c|c|c|c|}
\hline & $e e \mathrm{LS}$ & $\mu \mu \mathrm{LS}$ & $\mu e \mathrm{LS}$ \\
\hline \hline Luminosity & 6,5 & 6,4 & 6,5 \\
Electron ID & $0.6,0.5$ & $\mathrm{NA}, \mathrm{NA}$ & $0.6,0.6$ \\
Muon ID & $\mathrm{NA}, \mathrm{NA}$ & $1.5,1$ & $0.6,0.6$ \\
Lepton Fake Rate & $\mathrm{NA}, 4$ & $\mathrm{NA}, 4$ & $\mathrm{NA}, 2$ \\
Conversion Removal & $\mathrm{NA}, 12$ & $\mathrm{NA}, \mathrm{NA}$ & $\mathrm{NA}, 13$ \\
Theory Cross Section & 7,5 & 7,5 & 7,5 \\
\hline
\end{tabular}

deal with either physics or detector related backgrounds.

\subsection{Interpretation of the CDF results}

The observations are in agreement with the SM predictions therefore we combine the results to obtain an upper limit on the chargino-neutralino cross section. We first 


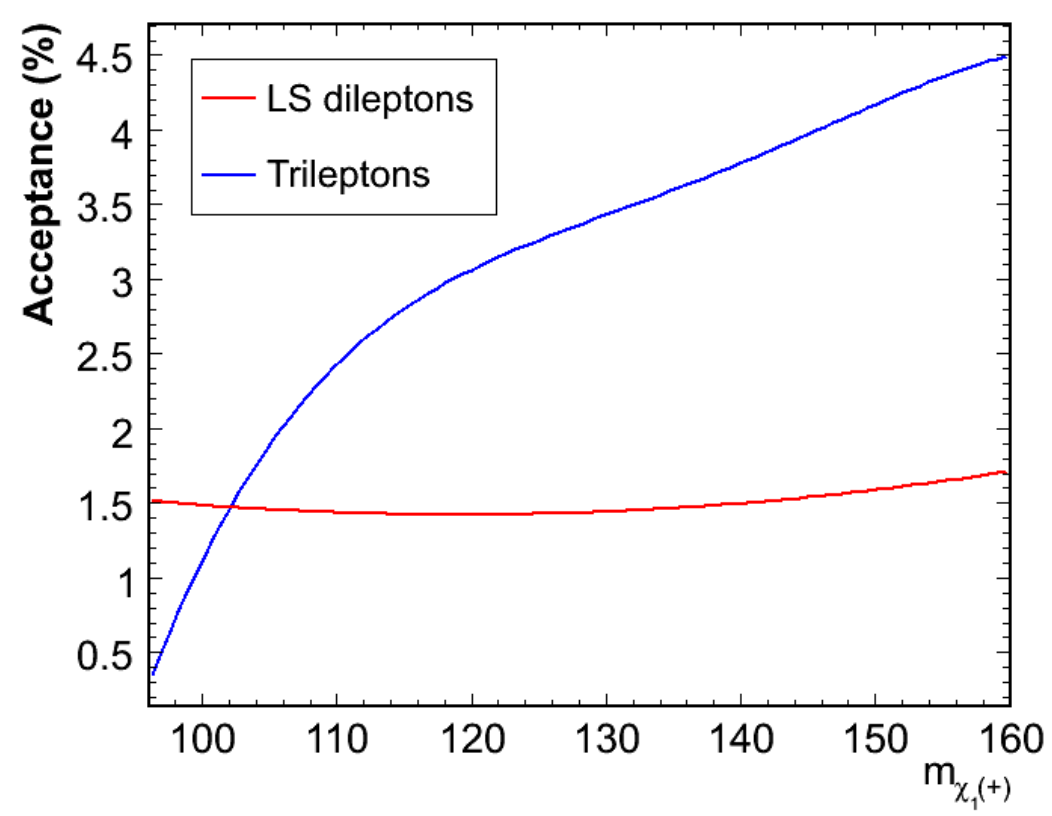

Figure 9.6. Acceptance of LS and trilepton analyses as a function of the chargino mass.

explore the upper limit in the mSUGRA scenario. Similarly to the limit calculation illustrated earlier, each channel is treated independently; the possible overlap between channels is taken into account utilizing the sensitivity $S / \sqrt{B}$ as a figure of merit to choose which analysis should include the shared SUSY event. The acceptance is recalculated accordingly. The exclusive background is obtained by rescaling the inclusive one by $\frac{A_{S U S Y}^{e x c l}}{A_{S U S Y}^{i n I}}$ where $A_{S U S Y}^{e x c l}\left(A_{S U S Y}^{i n c l}\right)$ is the exclusive (inclusive) acceptance for the SUSY signal. The calculation of the upper limit follows a frequentist approach [85] and incorporates the effect of the systematic uncertainties and correlations between channels and between signal and background for a given channel. The observed and expected limits are presented in Figure 9.7 in the mSUGRA scenario: based on this result, $\mathrm{CDF}$ is sensitive to masses up to $117 \mathrm{GeV} / c^{2}$, indicated by the expected limit; however, the observed mass limit is not competitive to the previous LEP II result.

In the mSUGRA like scenario however, the branching ratio into first and second 


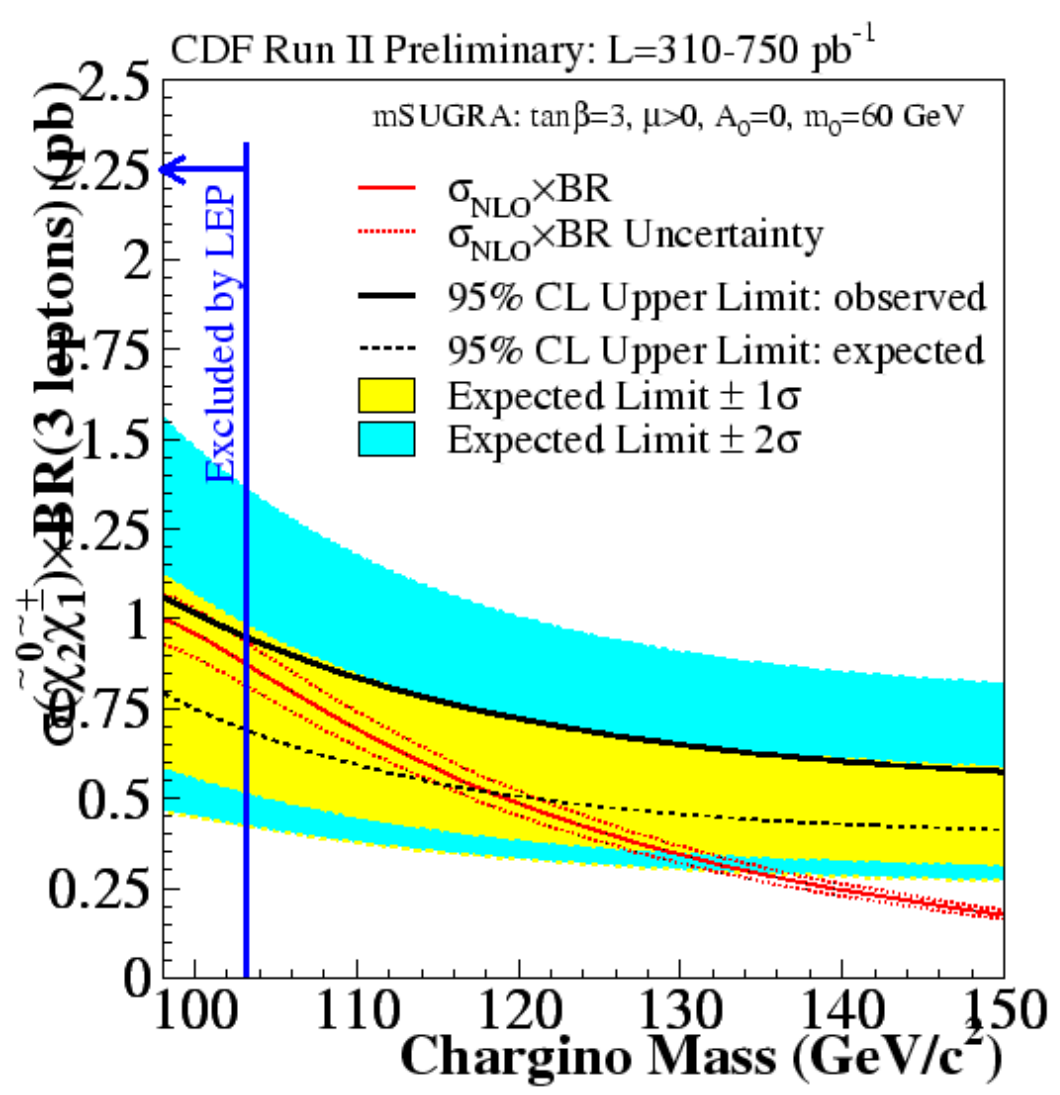

Figure 9.7. The excluded cross section limit is plotted as a function of the chargino mass in the mSUGRA scenario. The expected limit (black dashed line) and the theory curve (red solid line), with its uncertainty (red dotted lines) is also shown. The yellow and cyan bands represent the \pm 1 and \pm 2 sigmas uncertainties on the expected limit. The expected exclusion limit corresponds to a chargino mass of approximately $117 \mathrm{GeV} / c^{2}$.

generation leptons increases improving the sensitivity of our search. This is due to the fact that the slepton masses are degenerate, in particular the lightest $\tilde{\tau}$ is as heavy as the right-handed sleptons as shown in Figure 9.8 and Figure 9.9. As a consequence we expect the mass limit to be competitive with the previous mass limit set by LEP II. 


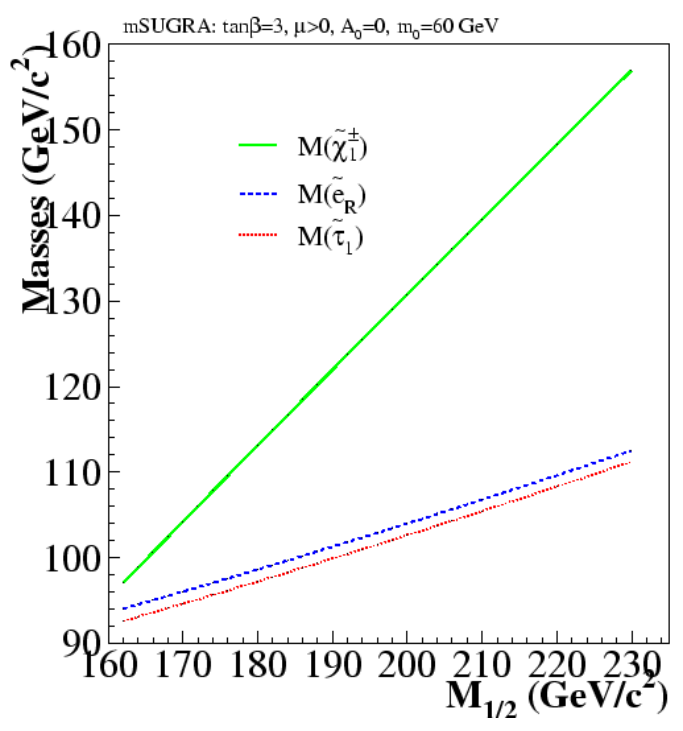

(a) mSUGRA scenario

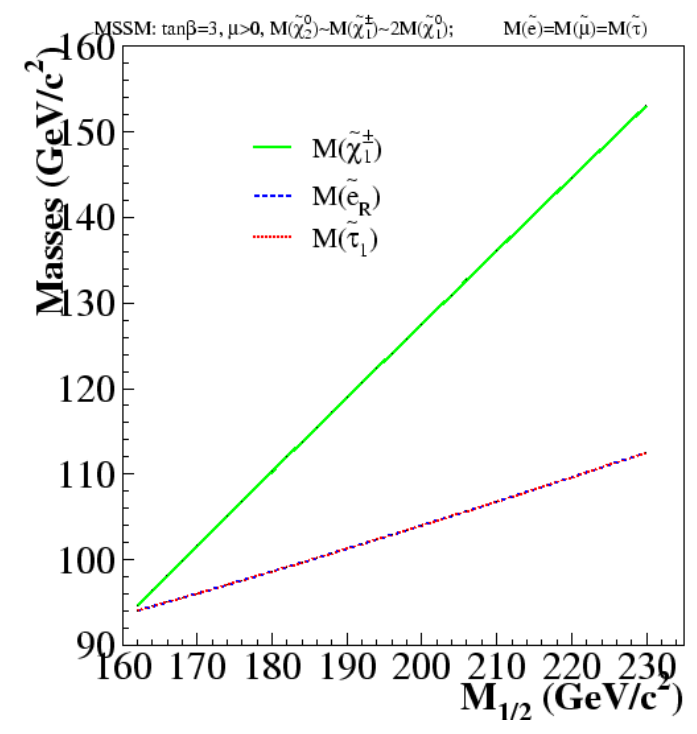

(b) mSUGRA like scenario

Figure 9.8. Masses as a function of the mSUGRA parameter $m_{\frac{1}{2}}$. 


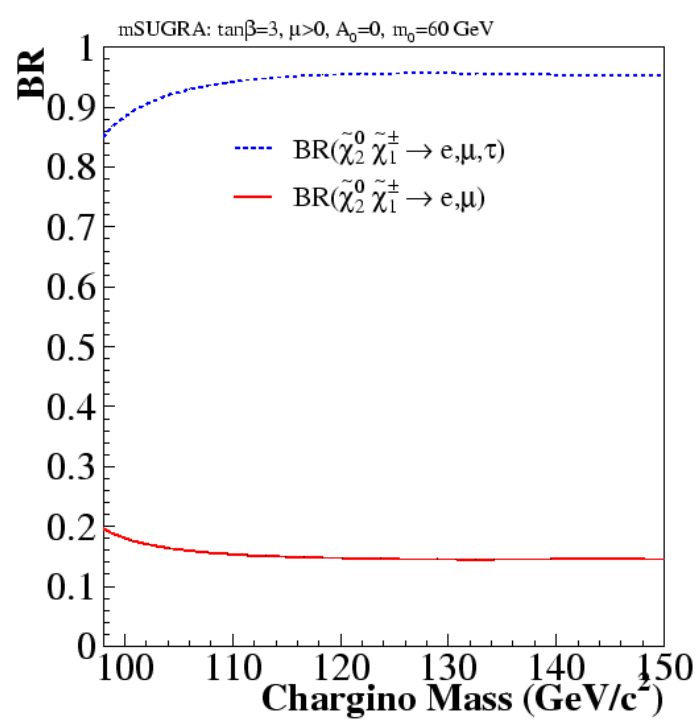

(a) mSUGRA scenario

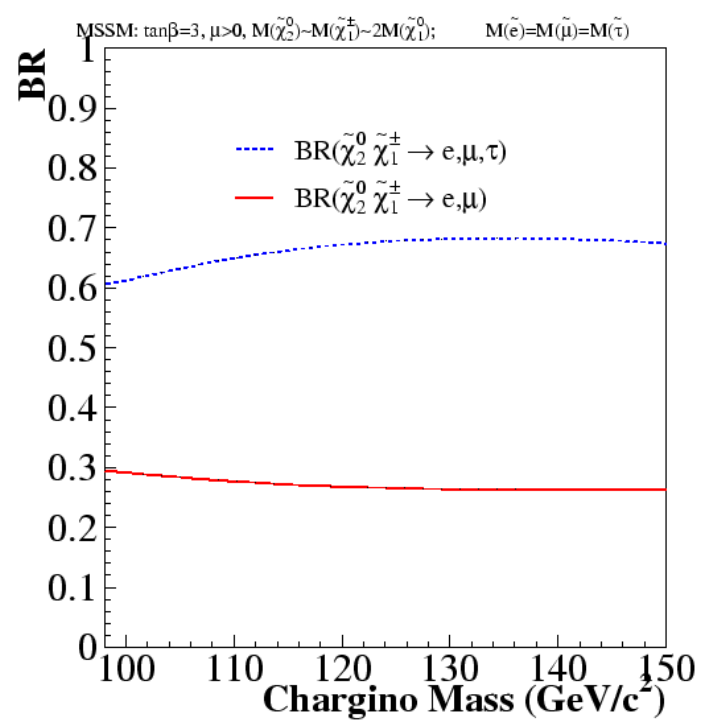

(b) mSUGRA like scenario

Figure 9.9. Branching ratios as a function of the mSUGRA parameter $m_{\frac{1}{2}}$. 
Both the observed and the expected limits in the mSUGRA like model are more stringent than the mSUGRA scenario and CDF can exclude a chargino mass up to $127 \mathrm{GeV} / c^{2}$ corresponding to a $\sigma \times B R$ of $\mathbf{0 . 2 5}$ as shown in Figure 9.10. In this case the sensitivity is up to masses of $140 \mathrm{GeV} / c^{2}$. The impact of the systematic

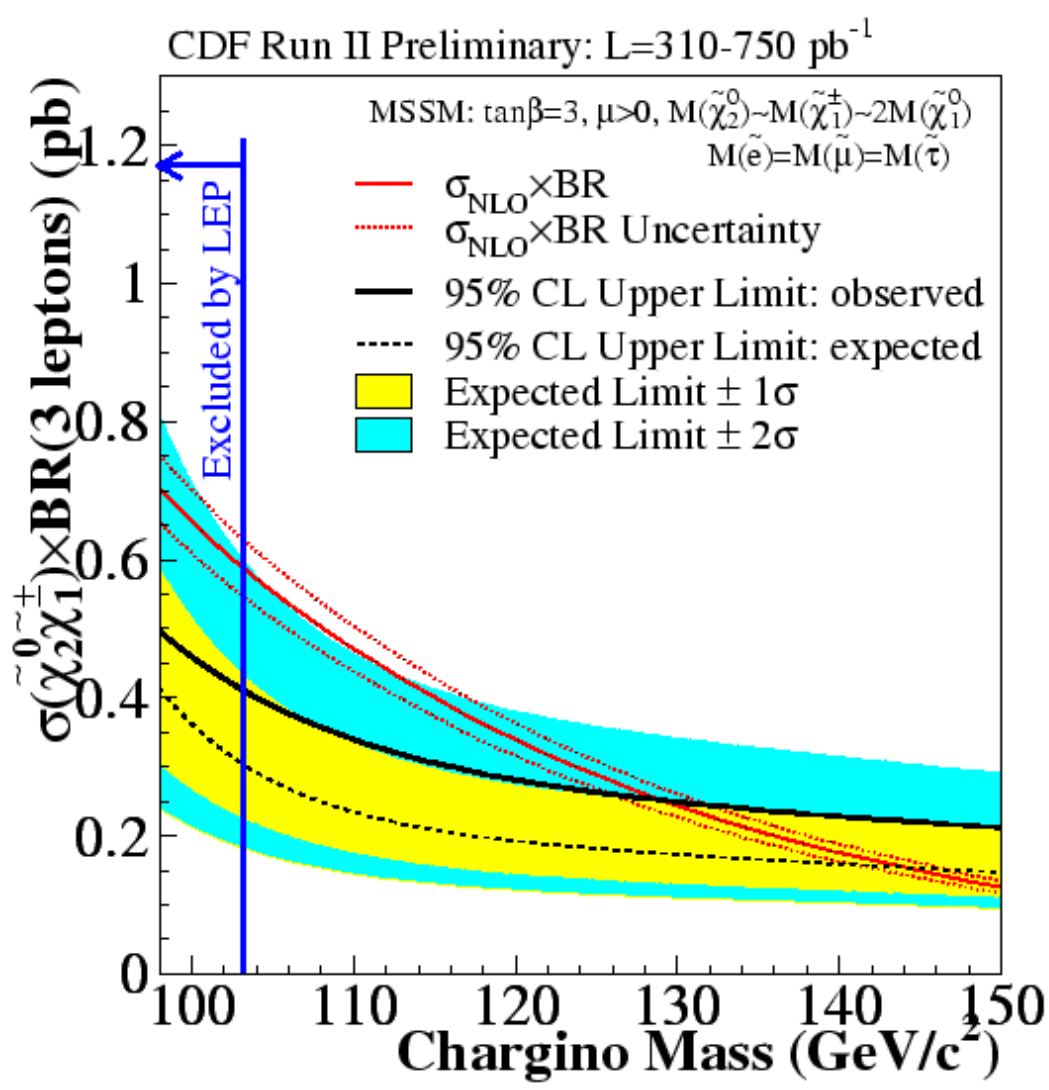

Figure 9.10. The excluded cross section limit plotted as a function of the chargino mass in a mSUGRA-like scenario with slepton mixing suppressed. The expected limit (black solid line), and the theory curve (red solid line) with its uncertainty (red dotted lines) is also shown. The yellow and cyan bands represent the \pm 1 and \pm 2 sigmas uncertainties on the expected limit. The observed limit on the mass of the chargino is approximately $127 \mathrm{GeV} / c^{2}$ and the expected one is approximately $140 \mathrm{GeV} / c^{2}$.

uncertainties on the upper limit value is estimated of the order of $3 \%$ [86]. 


\subsection{Conclusions}

We performed a search for the associated production of chargino and neutralino in up to $745 \mathrm{pb}^{-1}$ of data collected by the CDF experiment at the Tevatron $\mathrm{p} \overline{\mathrm{p}}$ collider. Events with at least three leptons and missing transverse energy larger than $15 \mathrm{GeV}$ are selected. We observed one event consistent with the expectations from the Standard Model backgrounds. The results of the search presented in this dissertation are combined with the results of similar analyses performed at CDF. As no evidence of SUSY production is observed, we set an upper limit on the chargino mass interpreting the result in the mSUGRA scenario where the slepton masses are degenerate. In this model chargino masses smaller than $127 \mathrm{GeV} / c^{2}$ are excluded. 
APPENDIX 


\section{A. Trigger efficiency}

The trigger efficiency is the probability that an event containing a muon satisfying the trigger requirements, defined as a candidate muon, fires the trigger. It can be calculated as the ratio of the number of events firing the trigger over the number of events events with at least one candidate muon. However, we are interested in a more generic approach. Instead of focusing on a given trigger path, we measure a lepton based efficiency. We determine the probability that a given muon satisfies the criteria at each trigger level. The aim is to provide a measurement suitable for several paths; in fact, different trigger paths can use different combinations of the same Level 1 (L1), Level 2 (L2) or Level 3 (L3) requirements.

In this context, the efficiency of the path is:

$$
\epsilon_{\mu}=\mathcal{P}(L 1 \mid R e c o) \times \mathcal{P}(L 2 \mid L 1) \times \mathcal{P}(L 3 \mid L 2)
$$

or

$$
\epsilon_{\mu}=\mathcal{P}(L 3 \mid \operatorname{Reco})
$$

where $\mathcal{P}(L 3 \mid$ Reco $)$ is the probability that a candidate muon fires the path provided that the muon has been reconstructed ${ }^{1}$, while $\mathcal{P}\left(L_{i} \mid L_{j}\right)$ is the $L_{j}$ conditional probability of the muon passing $L_{i}$. We perform the measurement in a so called "muon calibration sample", a data sample collected through a single muon trigger with a $p_{T}$ threshold of $8 \mathrm{GeV} / c$ and the requirement of both stubs in the CMU and CMP chambers. Since the trigger muon may bias the measurement of the CMUP trigger efficiency we remove it from the collection. To do so we match identified muons, called offline muons, which satisfy the trigger requirements, to the muons reconstructed at L3, called online muons [87]. The matching procedure depends upon the event topology:

\footnotetext{
${ }^{1}$ The muon reconstruction efficiency is larger than $90 \%$.
} 
- if there are two online CMUP8 muons in the event, we tag all offline muons as unbiased since the event would fire the trigger even if one of the online muon was not in the event;

- if there is one online CMUP8 muon in the event:

- the offline CMUP8 muon is matched to the online partner by requiring $\Delta R={\sqrt{\phi^{2}+\eta^{2}}}_{\min }<0.2 \mathrm{rad}$

- if the matching in the $\mathrm{R}$ space fails, the matching is performed in the $\phi$ space .

Figure A.1(a) and Figure A.1(b) show the transverse momentum of muons in the sample before and after the bias removal. Our procedure clearly suppresses any $p_{T}$ dependence. Once the trigger muon is identified and removed from the event the

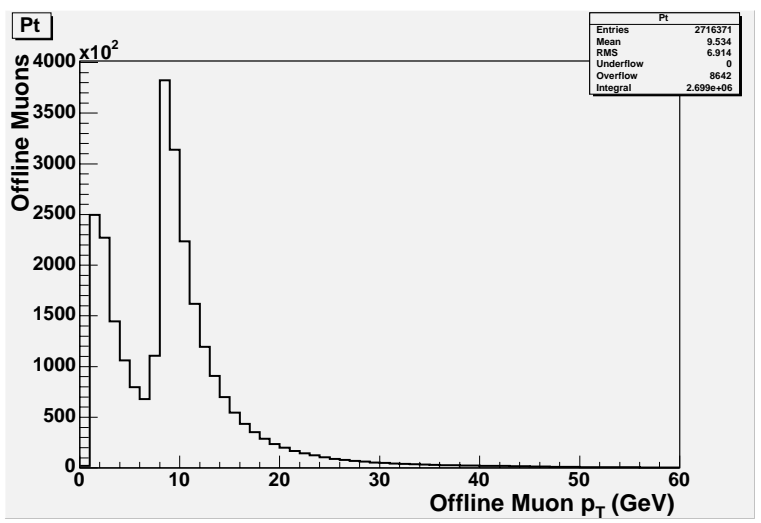

(a) Muon calibration data sample

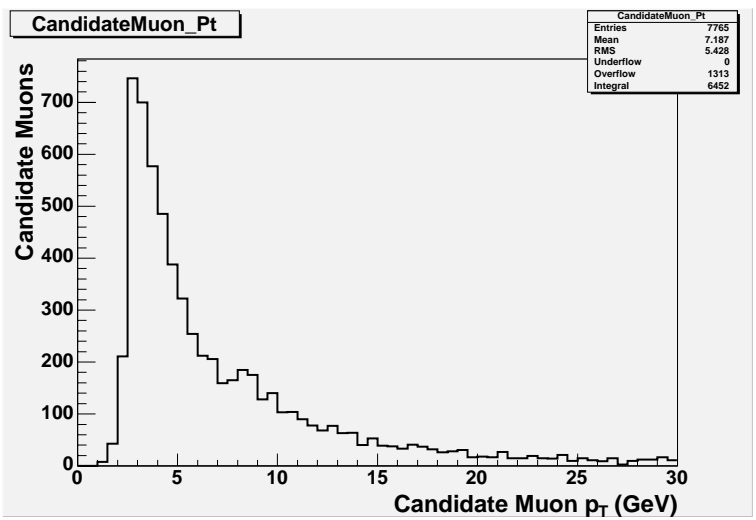

(b) Unbiased subsample

Figure A.1. Transverse momentum distribution of muons.

data sample can be used to measure the efficiency of the trigger paths of interest. We report the measurement of the L1 trigger efficiency measurement for the CMUP1.5 and CMUP6 triggers. The latter is the L1 in the paths used for the analysis ${ }^{2}$. 


\section{A.1 L1_CMU1.5_PT1.5}

The L1 trigger is based on a muon two stack tower with one pair of hits in every other layer. This stub is then associated to the XFT by the XTRP which projects the $\mathrm{XFT}$ with $p_{T}>1.5 \mathrm{GeV}$ into the muon chamber by estimating an angular range where the track could enter the chamber. A L1 muon trigger decision is released when one muon tower matches the XTRP projection. The trigger information is available in the data banks which record stubs found in the muon chamber and the trigger decision based on the $\mathrm{XTRP}^{3}$. The matching between the primitive, or online objects, and the offline muon is done using the information about the CMU stub and track associated to the offline muon and the information about the CMU stub, the XTRP projection and the XFT of the primitive. We associate the online low $p_{T}$ CMU stub to the low $p_{T}$ CMU extrapolation of the XFT through a bit wise comparison. Once the online stub is identified, it is matched to the offline CMU stub in the $\phi_{0}$ space. The matching efficiency, denoted in this case "stub" efficiency is $\epsilon_{s t u b}=92.64 \pm 0.37 \%$,

- L1_CMUP1.5_PT1.5

- CMU_STUB_PT $=1.5 \mathrm{GeV} / c$

- CMU_XFT_Pt $=1.5 \mathrm{GeV} / c$

- REQUIRE CMP $=1$

- L1_CMUP6_PT4

- CMU_STUB_PT $=6 \mathrm{GeV} / c$

- CMU_XFT_Pt $=4.09 \mathrm{GeV} / c$

- REQUIRE CMP $=1$

- L2_TRK8_L1_CMUP1.5_PT4

- NUMBER $=1$

- XFT_PT $=8 \mathrm{GeV} / c$

${ }^{3}$ There are 12 units, covering $30^{\circ}$ each, which contain four words for CMU and one word for CMUXTRP. Words $3 / 5(4 / 6)$ contain the position of the high (low) $p_{T}$ stub in the east/west side of the CMU; each word has 24 bit to cover $30^{\circ}$ ( 1 bit corresponds to a single $1.25^{\circ}$ stack); the L1 granularity, however is a $2.5^{\circ}$ tower, therefore we read 2 bits at the time and we convert the bit to the angular position simply by: $\phi_{\text {trigger }}=j_{\text {unit }} * 30+\left(i_{\text {bit }}+1.25\right) * 2.5$. The information about the XTRP projection is stored in word 33 which has 24 bits: the first 12 bits are used for the low $p_{T}$ and the following 12 bits for the high $p_{T}$. The granularity here is already $2.5^{\circ}$ so each bit has to be read and the conversion is done by the same expression as before. 
where the error is the statistical error only. As expected, the efficiencies in $\phi$ and $Z_{0}$ are flat within $0.2 \%$ [88]. The offline muon is associated to an XFT track with $p_{T}>1.5 \mathrm{GeV}$ in the $\phi_{0}$ space by selecting the muon closest to the online track and requiring $\Delta \phi<0.2 \mathrm{rad}$. The efficiency, averaged in $Z_{0}$ and in $\phi$ and denoted "track" efficiency has a plateau value $\epsilon_{t r k}=95.30 \pm 0.30 \%$ for offline $p_{T}>3 \mathrm{GeV}$. If the offline muon is matched to both a CMU-XTRP stub and a XFT track, this muon is labelled as L1 triggering muon and efficiency denoted $\epsilon_{L 1}$. A simple functional form is used to fit the efficiency with respect to the offline muon $p_{T}$ :

$$
\epsilon_{L 1}=\frac{A}{1+\exp \left[B \times\left(p_{T}+C\right)\right]}
$$

The result of the fit gives $\epsilon_{L 1}=93.09 \pm 0.54 \%$.

\section{A.2 L1_CMUP6_PT4}

The requirements of this trigger are different with respect to the low $p_{T}$ trigger in that the CMU stub has to be labelled high $p_{T}$ stub, associated to a high $p_{T}$ extrapolation of the XFT and to a CMP stub ${ }^{4}$. The matching between the online CMU stub and the extrapolation of the XFT is performed as before. In this case, the stub is also associated - through a bit wise comparison - to the CMP stub; given the multiple scattering and curvature of the muon, the association is performed up to an accuracy of 2.5 degrees. By measuring the minimal distance between the CMUP online stub and offline CMU stub, the offline muon is then matched to the online object and the corresponding efficiency is plotted in Figure A.2(a) with a plateau of $\epsilon=90.57 \pm 0.56 \%$. In case of the XFT track, the efficiency shown in Figure A.2(b) has a plateau value of $\epsilon=95.03 \pm 0.41 \%$. The efficiencies are flat in $\phi$ and $Z_{0}$ within the statistical uncertainty. In the same way as for the low $p_{T}$ trigger, if the offline muon is associated to both a CMUP stub and to an XFT, then it is tagged as a L1

${ }^{4}$ For CMP, we retrieve the high 8 bits of word 24 and the low 4 bits of word 26; we obtain a 12 bit word which covers $30^{\circ}$ (each bit corresponds to $2.5^{\circ}$ stack); there is no distinction neither between east and west nor between high and low $p_{T}$. 
triggering muon. The L1 efficiency can be found in Figure A.3(a) as a function of the offline muon $p_{T}$ and the result of the fit is $\epsilon=90.20 \pm 0.53 \%$.

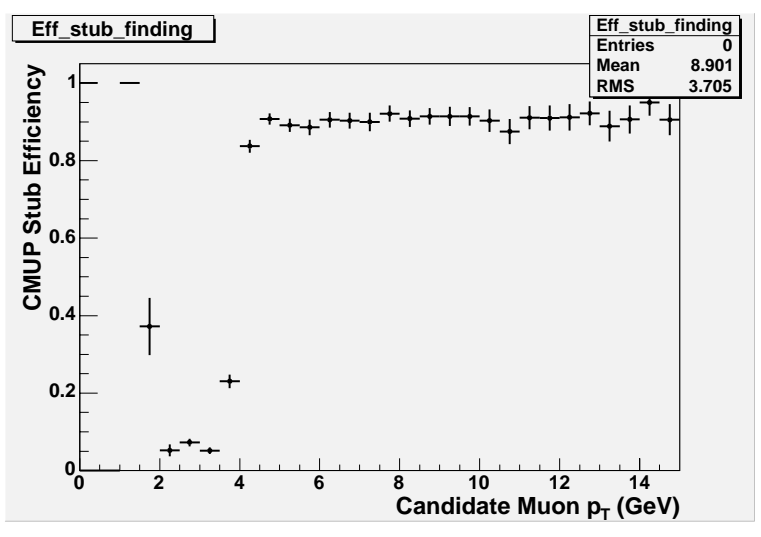

(a) CMU Stub

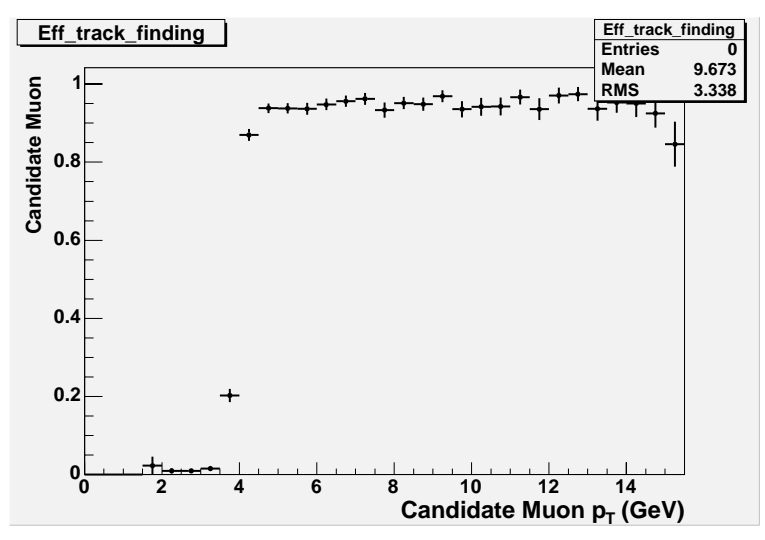

(b) XFT

Figure A.2. Matching efficiencies as a function of the offline muon $p_{T}$.



(a) $p_{T}$ dependence

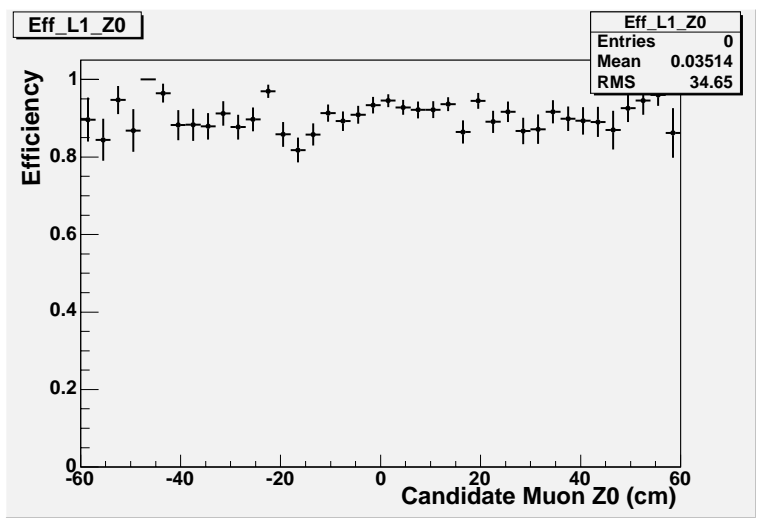

(b) $Z_{0}$ dependence

Figure A.3. L1 Trigger efficiency. 


\section{A.3 L2_TRK8}

Since the XFT block is cloned from the L1 bank without additional information, a L2 triggering muon is a L1 triggering muon with XFT $p_{T}>8 \mathrm{GeV}$. In Figure A.4(a) the probability $\mathcal{P}(L 2 \mid R e c o)$ is given with respect to the offline muon $p_{T}$ (the efficiency is defined as the probability that a candidate muon fires L1 and that same muon fires also L2). The limit value is $\epsilon=90.86 \pm 0.76 \%$. The $\mathcal{P}(L 2 \mid L 1$ is as expected almost $100 \%$ as shown in Figure A.4(b).



(a) L1

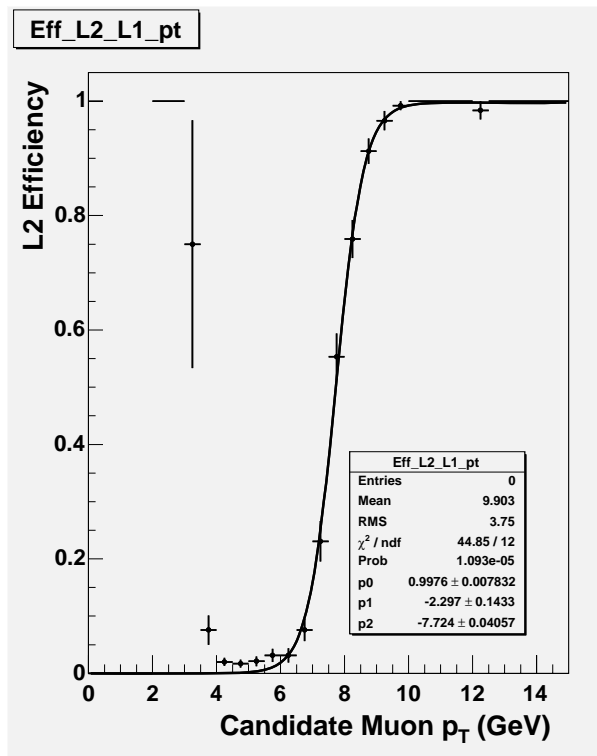

(b) L1 and L2

Figure A.4. Trigger efficiency as a function of the offline muon $p_{T}$.

More details about the trigger measurements can be found in [88]. 


\section{B. Muon identification efficiency}

The efficiency of identifying a muon is defined as the probability that a real muon satisfies the identification criteria. We collect real muons from the decay product of $J / \Psi, \Upsilon$ and Z; at this stage, the muons are just minimum ionizing tracks with the invariant mass in the appropriate range. The ratio between the number of muons satisfying the identification criteria and the total number of muons is the efficiency, which is measured in both the data and the Monte Carlo samples. The data are collected with a single muon trigger with a minimum $p_{T}$ threshold of $8 \mathrm{GeV} / c$ and muon stub requirements in either the CMX or the CMU-CMP chambers. The Monte Carlo $J / \Psi$ and $Z$ samples are generated with PYTHIA [67]. The efficiency measurement consists of several sequential steps. First, to avoid any bias on the efficiency measurement the muon which fired the trigger is excluded from the list of the muons the same way as in Appendix A. Once the trigger muons are identified, we proceed by selecting opposite sign muons in the mass range of the $J / \Psi, \Upsilon$ and Z. Even though the background contamination is expected to be small at high $p_{T}$, it is removed by subtracting the events in "side bands" around the resonances. Since the background is estimated as the average number of opposite sign muon pairs with invariant mass values below and above the resonance range, the efficiency is calculated as:

$$
\epsilon=\frac{N_{p}-0.5 N_{p}^{S B}}{N_{0}-0.5 N_{0}^{S B}}
$$

where $N_{p}\left(N_{p}^{S B}\right)$ is the number of muons passing the identification requirements and $N_{0}\left(N_{0}^{S B}\right)$ the total number of muons in the resonances (side bands). This is the definition of the total efficiency. When we evaluate the efficiency of a single cut $\mathrm{N}$, we apply the N-1 cuts, i.e.:

$$
\epsilon^{i}=\frac{N_{p}-0.5 N_{p}^{S B}}{N_{p-i}-0.5 N_{p-i}^{S B}}
$$


where $N_{p-i}$ is the number of muons passing all cuts except the cut labelled "i". The statistical errors are calculated using the formula [89]:

$$
\delta \epsilon=\sqrt{\frac{(1-2 \epsilon)\left(N_{p}+0.5^{2} N_{p}^{S B}\right)+\epsilon^{2}\left(N_{0}+0.5^{2} N_{0}^{S B}\right)}{N_{0}^{2}}},
$$

appropriate for efficiency smaller than 1. In Table B.1 we show an example of the results of our measurements. At medium $p_{T}$, the efficiency are mostly dominated by the isolation measured in [90] and the stub matching requirements. It is important to note that the efficiency depends on the status of the detector and the instantaneous luminosity. As a consequence, the results presented here are illustrative, since the values utilized in the analysis vary over the data taking period. In particular the threshold for the track $\chi^{2}$ itself is modified in time to maintain a constant efficiency of $\sim 99 \%$. The study was repeated using a slightly different event selection based on

Table B.1

Muon identification efficiency in data and MC. Note that the identification efficiency of medium $p_{T}$ muons does not include the loose isolation efficiency calculated in [90] and the stub matching requirement for the CMU is $\Delta X<3 \mathrm{~cm}$.

\begin{tabular}{|c|c|c|}
\hline Type & data & MC \\
\hline \hline CMUP & $96.76 \pm 0.48 \%$ & $96.34 \pm 0.08 \%$ \\
CMX & $97.34 \pm 0.82 \%$ & $96.27 \pm 0.11 \%$ \\
\hline Type & Iso Efficiency (data) & Iso Efficiency (MC) \\
\hline \hline CMUP/CMX/CMIO & $90.7 \pm 0.7 \%$ & $95.7 \pm 0.1 \%$ \\
\hline
\end{tabular}

a Drell-Yan MC sample which better reproduces the data. It is documented in [58]. 


\section{Photon conversion tagging efficiency}

Electrons originating from photons converting into $e^{+} e^{-}$pairs in the material represent a significant background for our analysis. Besides the decay products of $\pi^{0}$ and $\eta$, either internal or external bremsstrahlung contaminate any sample of prompt electrons ${ }^{1}$. If the photon from bremsstrahlung converts, a peculiar "trident" pattern, three tracks with small angular separation, could be observed in the detector. The contamination from photon conversion is reduced by using a "conversion tagging" algorithm. The algorithm matches the candidate electron to a close-by opposite sign track with at least one $\geq 5$ hit stereo and axial COT segments [91]. If $\Delta \cot \theta=\cot \theta_{t r k 1}-\cot \theta_{t r k 2}<0.02$ and the distance between the two tracks at closest approach is $D_{x y}<0.1 \mathrm{~cm}$, the electron is likely to originate from a photon conversion and is discarded. The tagging efficiency $\epsilon$ of this algorithm is measured in data and in Monte Carlo.

Due to the magnetic field along the direction of the beam line, the $\mathrm{e}^{+} \mathrm{e}^{-}$from photon conversions curve in opposite directions in the transverse plane while keeping approximately the same Z position measured at the CES plane. This feature allows us to predict the relative $\phi$ position of the electron with respect to the positron when the leptons reach the calorimeter. This method of selecting the photon conversion candidates is calorimeter based only and it does not require any tracking information. This is crucial given the asymmetric nature of photon conversions shown in Figure C.1. Due to this one of the tracks could be very low $p_{T}$ and not reconstructed by our tracking algorithm but it could still be large enough to reach the calorimeter.

\footnotetext{
${ }^{1}$ Bremsstrahlung refers to any radiation due to the acceleration of a charged particle. External bremsstrahlung occurs for electrons with energy above $50 \mathrm{KeV}$ such that the energy loss by radiation is larger than the loss by ionization. The internal bremsstrahlung refers to radiation emission during beta decay, resulting in the emission of a photon of energy less than or equal to the maximum energy available in the nuclear transition.
} 
We obtain a set of photon conversion candidate events from a data sample collected

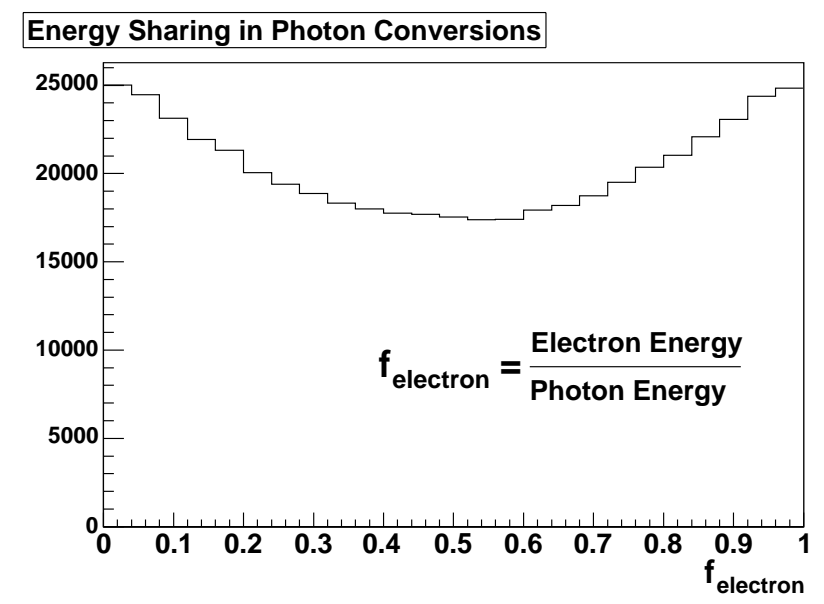

Figure C.1. Energy sharing between electron and positron in photon conversions.

with a single electron trigger with an $8 \mathrm{GeV} E_{T}$ threshold. The Monte Carlo samples are inclusive photon samples generated with the event generator PYTHIA [67] with a minimum photon $p_{T}$ of 8 and $12 \mathrm{GeV} / c$.

Given a seed electron we look for a partner CES cluster located up to $2 \phi$ wedges away from the seed wedge, not including the seed wedge itself. If there is more than one partner cluster than the one with the highest energy is selected. Depending on the charge of the seed electron, we define the neighboring wedge as either correct or incorrect as explained in Figure C.2. For example, if the charge of the seed electron is negative, we expect $\phi_{\text {seedEle }}>\phi_{C E S}$, whereas if it is positive, $\phi_{\text {seedEle }}<\phi_{C E S}$. If the seed electron-CES cluster pair is due to a conversion, we expect the partner cluster to be located in the correct wedge and the $\Delta Z$ between the seed electron and the CES cluster to be small. We select the conversion candidates by requiring $|\Delta Z|<$ $20 \mathrm{~cm}$. In Figure C.3 we show the distribution of $\Delta Z$ in the data and MC samples: when the partner cluster is found in the correct wedge, $\Delta Z$ peaks at small values. Distributions of the conversion candidate samples can be found in Figures C.4 and C.5.

In addition to photon conversions, there may be backgrounds which satisfy these 
selection criteria, such as

- electron accompanied by $\pi^{0}$ or $K^{ \pm}$

- electron accompanied by photons from minimum bias or underlying event (UE)

- jets $\left(\pi^{ \pm}+\left(\pi^{0} \rightarrow \gamma \gamma\right)\right)$

These components of the background are expected to be flat in $\Delta Z$, and can be estimated by the number of events in the incorrect wedge. However, bremsstrahlung $(e+\gamma)$ and trident events $\left(e+\left(\gamma \rightarrow e^{+} e^{-}\right)\right)$are not accounted for.

- Bremsstrahlung. In this case the electron curves while the photon propagates along the original electron direction and creates a CES cluster on the correct side as shown in Figure C.6. For most events we expect the electron to retain most of its original energy and therefore the photon should be relatively close to the electron. Since there is no track associated with the photon, such an event is not expected to be identified as a conversion.

- Trident. When the photon converts, either conversion electron may create a CES cluster. Typical trident events are shown in Figure C.7. If only the opposite sign electron is reconstructed then we expect the partner on the correct side and the seed to be tagged as a conversion. Otherwise if only the same sign electron


Figure C.2. Method of selecting conversion candidates and determining correct and incorrect side. 


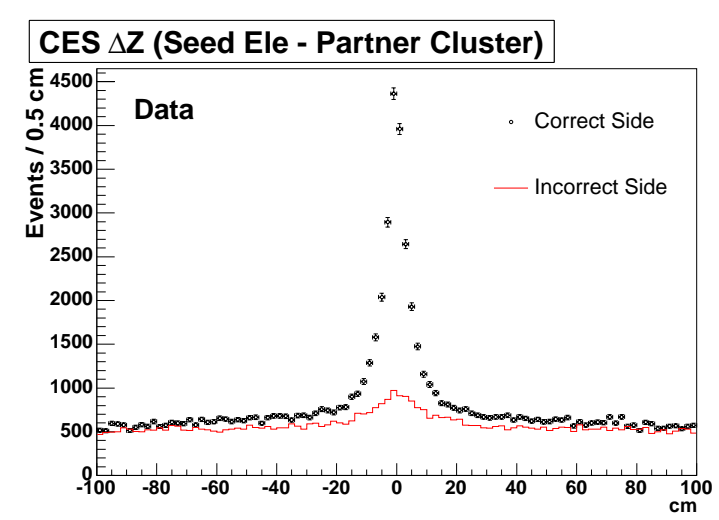

(a) Data Sample

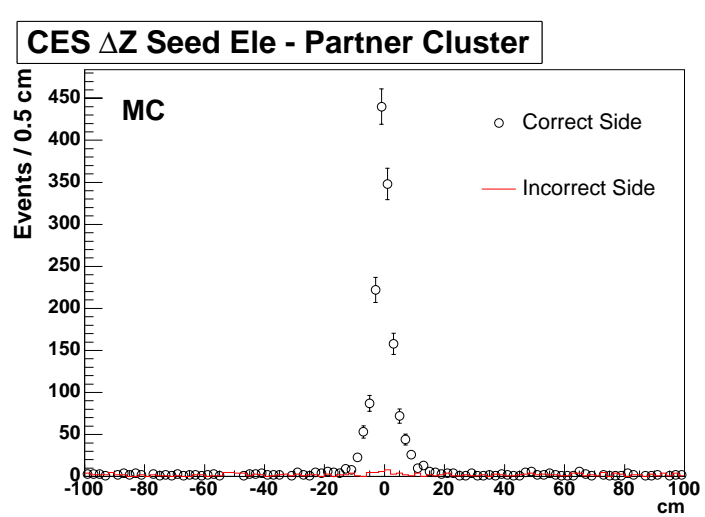

(b) MC Sample

Figure C.3. Local $\Delta \mathrm{Z}$ between the seed electron and the CES partner cluster for the correct (circles) and incorrect (red line) side.

is selected then the partner cluster could be on either side but the event should be untagged. In the scenario when both tracks are reconstructed we expect the event to be tagged regardless of which cluster is chosen.

To reduce the electron + photon background, in this study we apply cuts to reject electrons that do not originate from photon conversions. Since the probability of bremsstrahlung increases with the electron $p_{T}$, we place a cut on the missing energy $\left(\mathbb{E}_{\mathrm{T}}<15 \mathrm{GeV}\right)$ to remove $\mathrm{W}$ events and a cut on the dielectron invariant mass cut $\left(50<m_{e e}<106 \mathrm{GeV} / c^{2}\right)$ to reject $\mathrm{Z}$ events. Once the electron radiates a photon, the track $p_{T}$ decreases by the energy given off to the photon while the $E_{T}$ does not decrease since the calorimeter can not distinguish between the electron and the photon, resulting in an $E / p>1$. Therefore on top of the kinematic cuts, we also require the $E / p$ of the seed electron to be less than 1.1 to reduce the prompt electron background.

As a consequence the contamination from bremsstrahlung and trident events becomes negligible. This is confirmed by the absence of a narrow peak in the incorrect side (from MC simulated trident events we expect $\sigma_{M C} \sim 2.3 \mathrm{~cm}$ ), this indeed would 

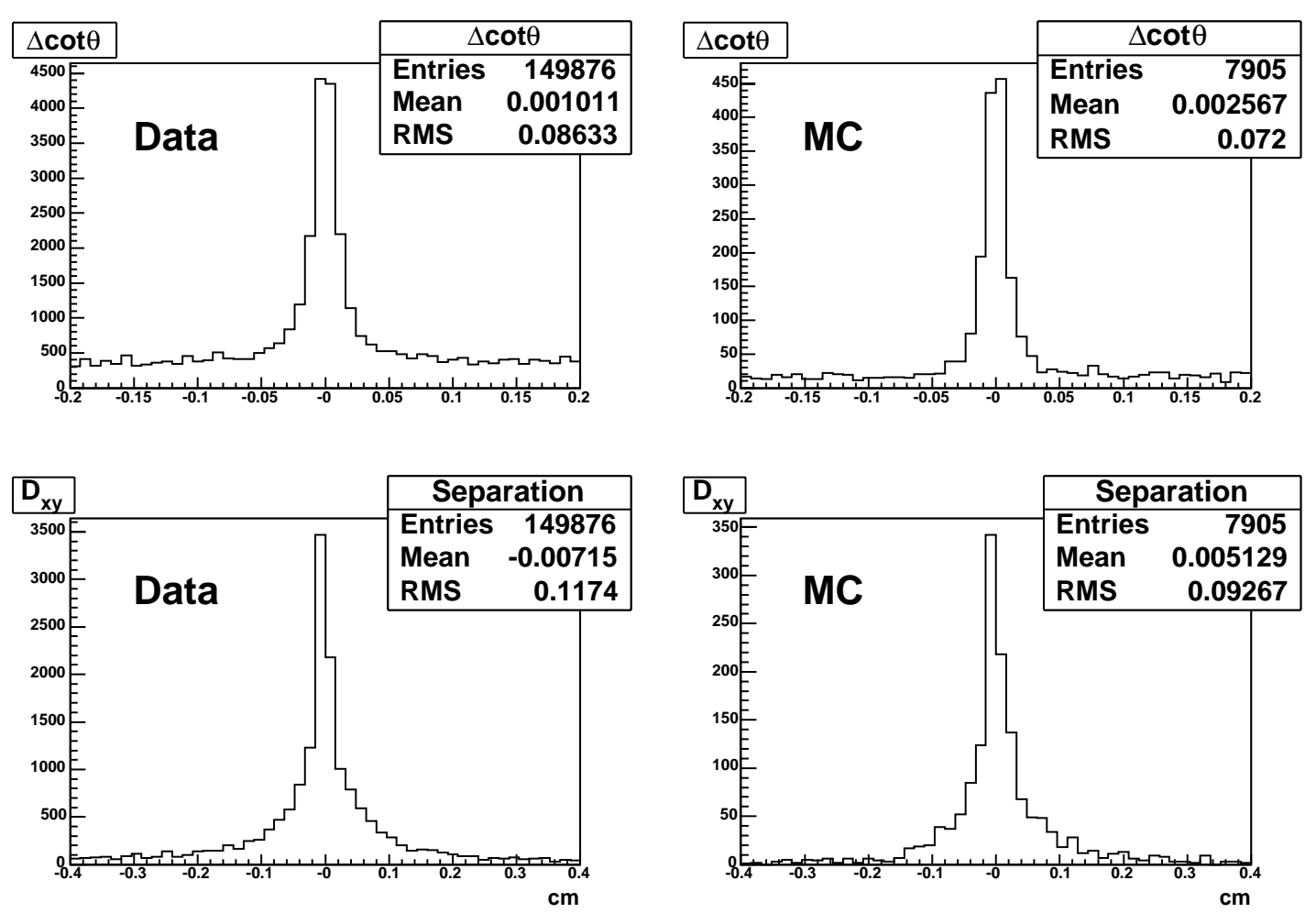

Figure C.4. Background subtracted $\Delta \cot \theta$ and $D_{x y}$ for Data and MC.

signal trident events. From Monte Carlo studies we inferred that the contribution from bremsstrahlung is smaller than the one from trident events.

As a result, the background can be removed by subtracting the incorrect side from the correct side. In particular the incorrect side is scaled up to take into account the difference in the tails of the data and MC distributions shown in Figure C.3. Before measuring the efficiency in data, an additional step is required. In fact, the conversion candidate sample (referred to as "CES sample") built so far is not inclusive since events where the partner electron does not create a CES cluster within two wedges from the seed electron are not selected. There are several reasons why the cluster could not be found:

- Electron $\mathrm{p}_{T}$ is small so that it reaches a $\phi$ wedge which is more than two wedges from the seed ( $a$ in Figure C.8). 

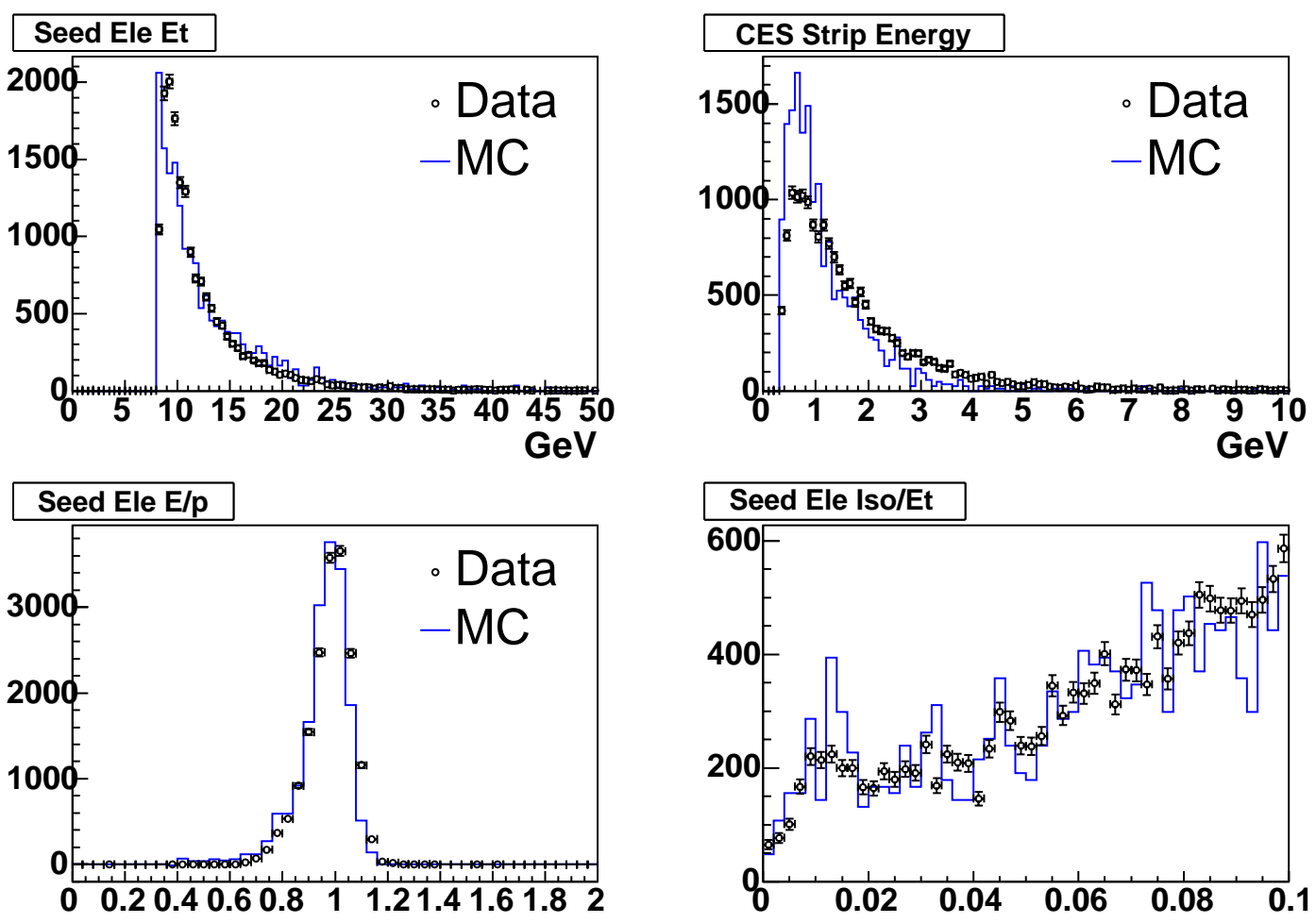

Figure C.5. Background subtracted Data and MC distributions of photon conversion candidates: seed electron transverse energy (top left), CES partner cluster energy (top right), seed electron $E / p$ (middle left), seed electron fractional isolation (middle right), $\Delta \cot \theta$ (bottom left), $\mathrm{D}_{x y}$ (bottom right).

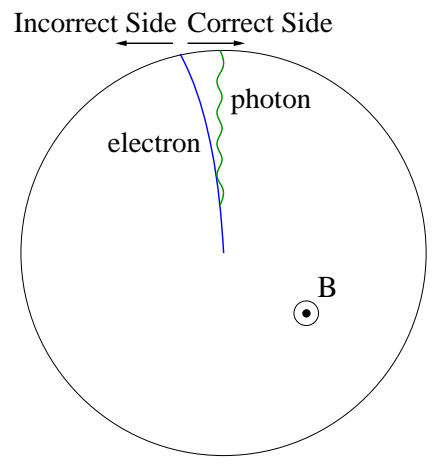

Figure C.6. A "bremsstrahlung" event where an electron radiates a photon. The photon will be on the correct side. 

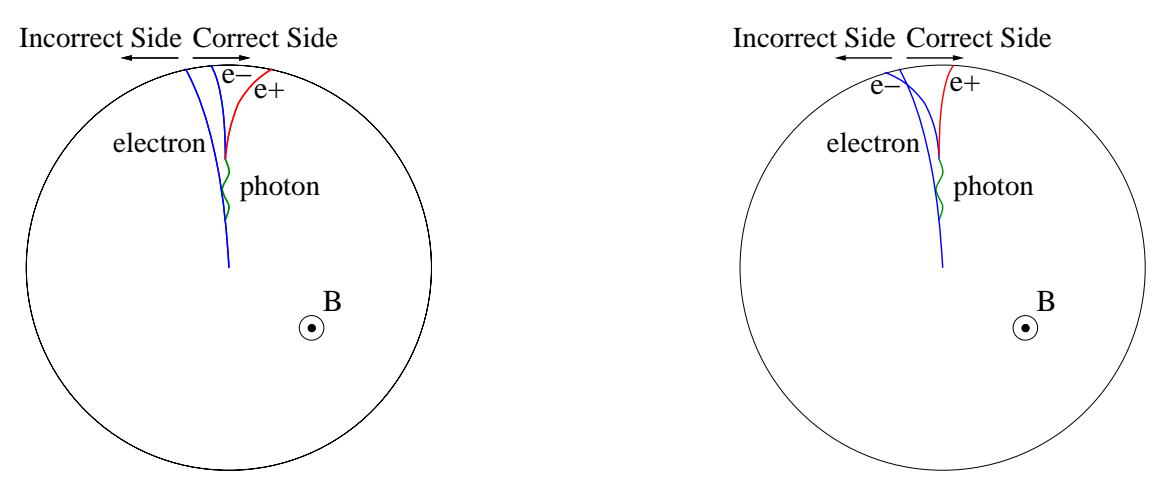

Figure C.7. Two "trident" events where an electron radiates a photon that converts.

- Electron $\mathrm{p}_{T}$ is so small that it never reaches the calorimeter ( $b$ in Figure C.8).

- Electron $\mathrm{p}_{T}$ is large enough so that the partner cluster is in the seed wedge $(c$ in Figure C.8).

In order to take this effect into account we proceed as follows. The sample of Monte Carlo conversions is split into two exclusive subsamples: the "CES sample" and the "non CES sample". The tagging efficiency is measured in both samples. As expected, the tagging efficiency of the "non CES sample" is lower than the efficiency measured in the CES sample. This is most likely due to lower tracking reconstruction for low $\mathrm{p}_{T}$ tracks since there must be at least two tracks for an electron to be tagged as a conversion. If the efficiency in the "non CES sample" is measured as a function of the partner track $p_{T}$, it is possible to determine a $p_{T}$ threshold such that the efficiency in the "non CES sample" equals the one in the "CES sample". The efficiency as a function of the minimum reconstructed $\mathrm{p}_{T}$ is shown in Figure C.9. Based on this plot we obtain the $\mathrm{p}_{T}$ threshold of $0.7 \mathrm{GeV}$. and we restrict the tagging algorithm to use only tracks above this $\mathrm{p}_{T}$ threshold. The results are shown in Table C.1. The conversion filter will be able to tag and reject more of these conversion events in $\mathrm{MC}$ than in data. By discarding tracks below a certain $\mathrm{p}_{T}$, the conversion background estimated from MC increases. 


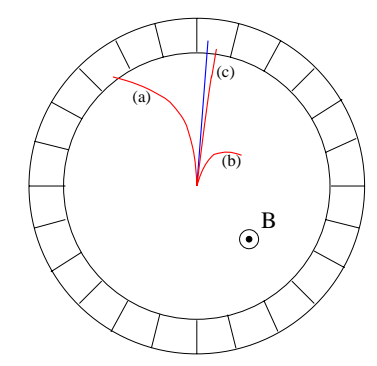

Figure C.8. CES clusters for photon conversions belonging to the "non CES sample": the blue line represents the seed electron, the red lines represent different scenario for the CES partner cluster (Monte Carlo events).

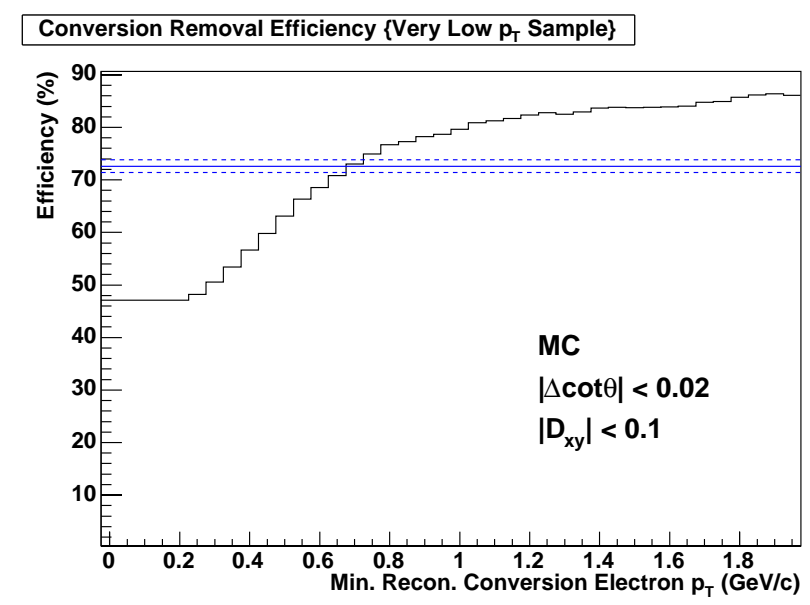

Figure C.9. Efficiency for the "non CES sample" as a function of the partner track minimum $\mathrm{p}_{T}$. The line represents the efficiency of the "CES sample".

Table C.1

Conversion removal efficiency in Data and MC.

\begin{tabular}{|c|c|c|c|}
\hline$\Delta \cot \theta$ & $\mathrm{D}_{x y}$ & $\epsilon_{\text {Data }}$ & $\epsilon_{M C}$ \\
\hline \hline 0.02 & 0.1 & $59.4 \pm 0.4 \%$ & $66.9 \pm 1.2 \%$ \\
0.04 & 0.2 & $72.2 \pm 0.4 \%$ & $79.7 \pm 1.0 \%$ \\
\hline
\end{tabular}


In our search the background due to electrons from $\gamma$ conversions is estimated from MC. Therefore a scale factor measured as:

$$
S F=\frac{1-\epsilon_{D A T A}}{1-\epsilon_{M C}}
$$

needs to be applied in order to have a realistic estimate from the MC in use. The scale factor measured as a function of the seed electron $\mathrm{E}_{T}$ (Figure C.10) will be applied to the MC photon conversion events that are not removed by the conversion tagger. We observe that the MC models the data at high values of electron energy and we use a scale factor equal to 1 for electrons with $E_{T}>40 \mathrm{GeV}$.

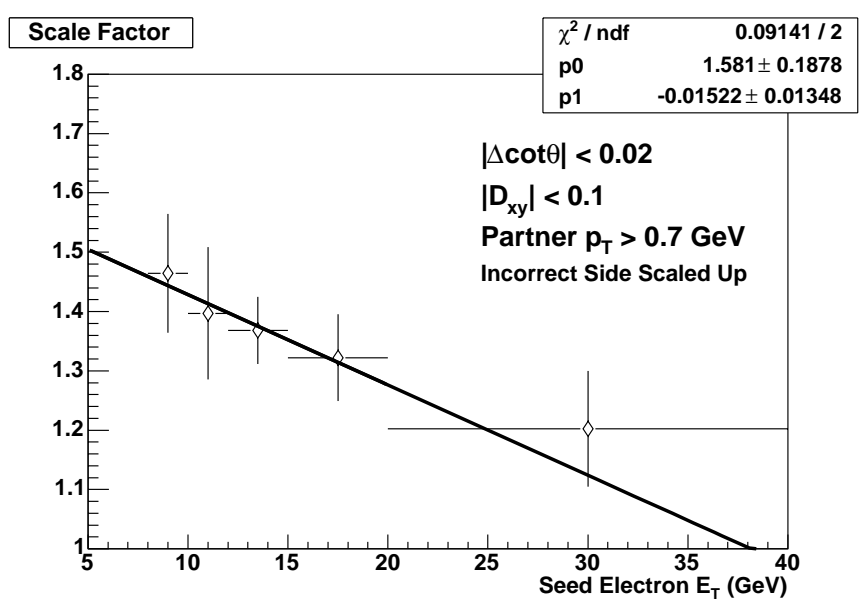

Figure C.10. Conversion removal scale factor as a function of the seed electron $\mathrm{E}_{T}$. Only the first $4 \mathrm{E}_{T}$ bins are used in the fit. The last bin is not used since the mean $\mathrm{E}_{T}=25 \mathrm{GeV}$ does not equal the bin center and would bias the fit.

The systematic uncertainties affecting the conversion removal efficiency are:

- Choice of partner track $p_{T}$ threshold. We estimate the systematic uncertainty due to the choice of the $\mathrm{p}_{T}$ threshold $=0.7 \mathrm{GeV}$ by measuring the scale factor for minimum $\mathrm{p}_{T}$ values of $0.6 \mathrm{GeV}$ and $0.8 \mathrm{GeV}$. The uncertainty per $\mathrm{E}_{T}$ bin is defined as: $\delta_{\text {scalefactor }}=\left(S F^{0.6}-S F^{0.8}\right) /\left(2 * S F^{0.7}\right)$. Averaging over all the $\mathrm{E}_{T}$ bins we get: $\delta_{\text {scalefactor }}=12.7 \%$. 
- Choice of $\Delta \mathrm{Z}$ cut. To estimate the uncertainty due to the $\Delta Z$ cut, we measure the scale factor for values above and below the nominal value of $20 \mathrm{~cm}$. The uncertainty is calculated as: $\delta_{\text {scalefactor }}=\left(S F^{25}-S F^{15}\right) /\left(2 * S F^{20}\right)=3.3 \%$.

- Background subtraction. The incorrect side is scaled up to account for additional background. This represents an uncertainty in our understanding of the background. To account for this, we vary the scale up factor by $10 \%$ and observe how the conversion removal scale factor changes. $\delta_{\text {scalefactor }}=$ $\left(S F^{\text {scale } * 0.9}-S F^{\text {scale } * 1.1}\right) /\left(2 * S F^{\text {scale } * 1.0}\right)=19.6 \%$.

- CES cluster reconstruction efficiency at low energies. We estimate the systematic uncertainty due to the CES reconstruction efficiency by comparing the scale factors for photon candidates with CES cluster $\mathrm{E}>0.7 \mathrm{GeV}$ and $\mathrm{E}<0.7 \mathrm{GeV}$. We obtain: $\delta_{S F}=S F^{E>0.7}-S F^{E<0.7}=5.4 \%$.

- Eta dependence. We expect the scale factor to be the same for conversions in the east and west sides. Any difference is taken as a systematic error: $\delta_{\text {scalefactor }}=$ $\left(S F^{\text {East }}-S F^{\text {West }}\right) /\left(2 * S F^{\text {Nominal }}\right)=15.7 \%$.

The total systematic uncertainty is $\delta_{\text {scalefactor }}=28.8 \%$. Details about the measurements can be found in [92]. 
LIST OF REFERENCES 


\section{LIST OF REFERENCES}

[1] S. Weinberg. Phys.Rev.Lett, 19:1264, 1967.

[2] A. Salam. Elementary particle theory. $8^{\text {th }}$ Nobel Symposium, 1968.

[3] L. Maiani J. Iliopoulos, S. L .Glashow. Phys.Rev., D 2:1285, 1970.

[4] A. J. Macfarlane. Dirac matrices and the Dirac matrix description of Lorentz transformations. Comm.Math.Phys., 2, 1966.

[5] N. Cabibbo. Unitary Symmetry and Leptonic Decays. Phys.Rev., 10:531-533, 1963.

[6] T. Maskawa M. Kobayashi. CP violation in the renormalizable theory of weak interaction. Prog.Theor.Phys, 1973:652-657, 49.

[7] Weinberg et al. Phys.Rev., D 66:919, 2002.

[8] W. Higgs. Phys.Lett, 12, 1964.

[9] S. P. Martin. A Supersymmetry Primer. hep-ph/9709356, 1997.

[10] M. Drees. An introduction to Supersymmetry. hep-ph/9611409, 1996.

[11] H. Murayama. Supersymmetry Phenomenology. hep-ph/0002232, 2000.

[12] J. D. Lykken. Introduction to Supersymmetry. hep-th/9612114, 1996.

[13] M. Carena et al. The Search for Supersymmetry at the Tevatron Collides. hepph/9712022, 2002.

[14] C. Quigg. Gauge theories of the strong, weak and electromagnetic interactions. Addison-Wesley Publishing Company, 1983.

[15] M. Peskin. Beyond the Standard Model. hep-ph/9705479, 2002.

[16] S. Eidelman et al. Phys. Lett., B:592, 2004.

[17] Bagger. Weak scale supersymmetry: theory and practice. hep-ph/9604232, 1996.

[18] Bagger Wess. Supersymmetry and supergravity. Princeton University, 1992, Press Princeton, NJ, 2nd edition.

[19] T.Plehn. http://www.ph.ed.ac.uk/ tplehn/.

[20] WMAP Collaboration. http://map.gsfc.nasa.gov/. 
[21] S. Khalil. Relic Neutralino Density in Scenarios with Intermediate Unification Scale. New J.Phys., 4, 2002.

[22] H.Baer et al. Updated constraints on the minimal supergravity model. hepph/0210441, 2002.

[23] F. Perez. On supersymmetric Dark Matter. 2000.

[24] G. Weiglein et al. Physics interplay of the LHC and the ILC. hep-ph/0410364.

[25] The D0 Collaboration. First Direct Two-Sided Bound on the Bs0 Oscillation Frequency. hep-ex/0603029, 2006.

[26] The CDF Collaboration. Measurement of the Bs-Bsbar Oscillation Frequency. hep-ex/0606027, 2006.

[27] http://www.utfit.org.

[28] http://ckmfitter.in2p3.fr.

[29] E.Lunghi et al. Analysis of enhanced $\tan \beta$ effects in MVF GUT scenarios. hepph/060517\%.

[30] M. Carena et al. Constraints on B and Higgs physics in minimal low energy supersymmetric models. hep-ph/0603106, 2006.

[31] The CDF Collaboration. Search for FCNC Rare Decay: Bs into $\mu \mu$. Public, CDF8176.

[32] Buras et al. Phys.Lett., B, 2003.

[33] Belle Collaboration. Phys.Rev., B 511:151, 2001.

[34] Belle Collaboration. Phys.Rev.Lett, 93:061803, 2004.

[35] Babar Collaboration. Phys.rev., D72, 2005.

[36] Babar Collaboration. hep-ex/0507001.

[37] CLEO Collaboration. Phys.Rev.Lett, 87:251807, 2001.

[38] T. Hurth et al. Nucl.Phys., B 704:56, 2005.

[39] http://www.g 2.bnl.gov/index.shtml.

[40] R.Arnowitt et al. Phys.Lett, B 538:121, 2002.

[41] Ellis et al. Indications of the CMSSM Mass Scale from Precision Electroweak Data. hep-ph/0604180.

[42] H.Baer et al. Reach of the Fermilab Tevatron for minimal supergravity in the region of large scalar masses. hep-ph/0305325.

[43] http://lepsusy.web.cern.ch/lepsusy/.

[44] http://www.cpt.dur.ac.uk/ georg/sps/. 
[45] The CDF Collaboration. The CDF II Detector. Technical Design Report. 1996.

[46] W. Budgett et al. Description of CDF Data Structure for Run II. Internal, CDF4152.

[47] Grundler et al. High $p_{T}$ muons recommended cuts and efficiencies for Summer 2006. Internal, CDF8262.

[48] Konigsberg at al. Average cross-section of the inelastic $p \bar{p}$ scattering at $1.8 \mathrm{TeV}$. Internal, CDF6314, 2003.

[49] http://www-cdf.fnal.gov/internal/dqm/goodrun/good.html.

[50] C. Hays et al. Internal, CDF6992, 2004.

[51] P. Gatti. Performance of the new tracking system at CDF II. Internal, CDF5561, 2001.

[52] L. Nodulman. Curvature Corrections for 5.3.1. Internal, CDF6971.

[53] B. Heinemann et al. The z-Vertex Algorithm in Run II. Internal, CDF6238, 2002.

[54] W. Sakumoto. Event Cut Acceptance for Run II. Internal, CDF8318.

[55] K. Bloom et al. Muon Reconstruction Efficiency. Internal, CDF6029.

[56] A. Taffard. RunII Cosmic Ray Tagger Performances . Internal, CDF6255, 2003.

[57] C. Hays et al. Cosmic Ray Tagging using COT Hit Timing. Internal, CDF6089, 2002.

[58] A. Canepa et al. Intermediate pt muon ID efficiencies with the $1 \mathrm{fb}-1$ dataset. Internal, CDF8336.

[59] R .G. Wagner. Electron Identification for Run II: Algorithms. Internal, CDF5456, 2000.

[60] Spreitzer et al. Electron Identification in Offline Release 6.1.2. Internal, CDF7950.

[61] Dube et al. Low Et electron ID efficiency and scale-factors using J/Psi. Internal, CDF7379.

[62] Attal et al. ID Efficiencies for Medium $E_{T}$ Plug Electrons. Internal, CDF7345.

[63] A. Batti. DSTOWE. Internal, CDF3952, 2000.

[64] B. Flaughter. A GUIDE TO JETCLU: THE CDF JET CLUSTER ALGORITHM. Internal, CDF1814, 1992.

[65] Anwar Bhatti Florencia Canelli CDF Collaboration, Jet Energy Group. Jet Energy Corrections at CDF. hep-ex/0510047, 2005.

[66] A. Pompos. Search for the Scalar Top in Dilepton Events Produced in par p collisions at sqrt s=1.8 TeV. Internal, CDF6294, 2003. 
[67] Sjstrand et al. PYTHIA. http://www.thep.lu.se/torbjorn/Pythia.html.

[68] F. Maltoni and T. Stelzer. MadGraph. http://madgraph.hep.uiuc.edu/.

[69] J.M. Campbell et al. Update on vector boson production at hadron colliders. Phys.Rev., D:113006, 1999.

[70] M. Cacciari et al. JHEP, 2004.

[71] CDF Collaboration. Measurement of the ttbar Production Cross Section in pp-bar Collisions at sqrt $\mathrm{s}=1.96 \mathrm{TeV}$ Using Lepton Plus Jets Events with Semileptonic B Decays to Muons. Phys. Rev, D, 2005.

[72] Griffiths et al. Fake Rate For Low $p_{T}$ Leptons. Internal, CDF7470, 2005.

[73] H. Baer et al. ISAJET A Monte Carlo Event Generator for p p, pbar p, and e+ e- Interactions . http://www.phy.bnl.gov/isajet/.

[74] W. Sakumoto. W/Z Cross section predictions for $\sqrt{s}=1.96 \mathrm{TeV}$. Internal, CDF6341, 2003.

[75] J. Pumplin et.al. CTEQ6 PDF's. http://user.pa.msu.edu/wkt/cteq/cteq6/cteq6pdf.html.

[76] Stirling Thorne, Martin. MRST Parton Distributions - status 2006. hepph/0606244, 2006.

[77] http://mcfm.fnal.gov/.

[78] L. Lyons. Statistics for Particle and Nuclear Physicists. Cambridge University Press, ISBN 0521379342, 1986.

[79] J. Conway. Calculations of Cross Section Upper Limits Combining Channels Incorporating Correlated and UncorrelatedSytematic Uncertainties. Internal, CDF6428, 2005.

[80] J. Heinrich for the CDF Statistics Committee. Limit calculations in the presence of nuisance parameters. 1. Bayesian approach. Internal, CDF7117, 2004.

[81] The CDF Collaboration. Search for production of chargino and neutralino in the ee+lepton final states. Public, CDFr750, 2005.

[82] The CDF Collaboration. Search for production of chargino and neutralino in the $\mu \mu+$ lepton decay channel in $312 \mathrm{pb}^{-1}$. Public, CDF8273, 2006.

[83] The CDF Collaboration. Search for production of chargino and neutralino production in mSUGRA model in dielectron plus track channel. Public, CDF8186, 2006 .

[84] The CDF Collaboration. A search for new physics in like sign dileptons. Public, CDF8117, 2006.

[85] T. Junk. Confidence level computation for combining searches with small statistics. Nucl.Instrum.Meth., A434:435:443, 1999.

[86] The CDF Collaboration. Combined Limit for the Trilepton Analyses-Winter 2006. Public, CDF8310, 2006. 
[87] G. De Lentdecker et al. The L3Summary Object. Internal, CDF6424, 2003.

[88] A.Canepa et al. Trigger Efficiency for medium pT Dimuon Triggers in Run II. Internal, CDF7196, 2004.

[89] C. Blocker. Uncertainties on Efficiencies. Internal, CDF7168, 2004.

[90] Rekovic Gold. Isolation Efficiencies (MC and DATA). Internal, CDF7432.

[91] D. Gerdes. Study of Conversion Removal for Lepton + Jets Sample. Internal, CDF2903.

[92] A. Canepa et al. Photon Conversion Removal Efficiency. Internal, CDF8073, 2006. 